\title{
Ion Temperatures in Earth's Inner Magnetosphere: Ring Current Dynamics, Transient Effects, and Data-Model Comparisons
}

Justin G. Elfritz

West Virginia University

Follow this and additional works at: https://researchrepository.wvu.edu/etd

\section{Recommended Citation}

Elfritz, Justin G., "Ion Temperatures in Earth's Inner Magnetosphere: Ring Current Dynamics, Transient Effects, and Data-Model Comparisons" (2014). Graduate Theses, Dissertations, and Problem Reports. 563.

https://researchrepository.wvu.edu/etd/563

This Dissertation is protected by copyright and/or related rights. It has been brought to you by the The Research Repository @ WVU with permission from the rights-holder(s). You are free to use this Dissertation in any way that is permitted by the copyright and related rights legislation that applies to your use. For other uses you must obtain permission from the rights-holder(s) directly, unless additional rights are indicated by a Creative Commons license in the record and/ or on the work itself. This Dissertation has been accepted for inclusion in WVU Graduate Theses, Dissertations, and Problem Reports collection by an authorized administrator of The Research Repository @ WVU.

For more information, please contact researchrepository@mail.wvu.edu. 


\title{
Ion Temperatures in Earth's Inner Magnetosphere: Ring Current Dynamics, Transient Effects, and Data-Model Comparisons
}

\author{
Justin G. Elfritz \\ Dissertation submitted to the College of Arts and Sciences \\ at West Virginia Univeristy \\ in partial fulfillment of the requirements \\ for the degree of \\ Doctor of Philosophy \\ in \\ Physics
}

\author{
Amy M. Keesee, Ph.D. Chair \\ Loren Anderson, Ph.D. \\ Paul A. Cassak, Ph.D. \\ John Christian, Ph.D. \\ Earl E. Scime, Ph.D. \\ Department of Physics and Astronomy \\ Morgantown, West Virginia
}

2014

Keywords: magnetosphere, space physics, ion heating, ion temperatures, computational physics, plasma physics

Copyright 2014 Justin G. Elfritz 


\title{
ABSTRACT \\ Ion Temperatures in Earth's Inner Magnetosphere: Ring Current Dynamics, Transient Effects, and Data-Model Comparisons
}

\author{
Justin G. Elfritz
}

Earth's magnetosphere is an inherently complex, strongly nonlinear system with intrinsic coupling between internal and external drivers. In general, magnetospheric systems can be understood as a balance between multiple regions which all exhibit unique plasma properties. The feedback processes between each region depend on geomagnetic activity levels and the preceding states of the solar wind and the respective magnetospheric regions. Of particular interest is understanding how ion temperatures evolve during geomagnetically active periods, and also understanding the space weather impacts of hot ion populations injected during such periods. Dynamic, spatiotemporally resolved ion temperature boundary conditions have been implemented into the Comprehensive Ring Current Model (CRCM); the temperatures are based on 2-D equatorial maps derived from remotely imaged energetic neutral atom (ENA) measurements. The high-speed-stream-driven event on 22 July 2009 and the coronal mass ejection-driven event on 30-31 October 2013 are simulated and compared against identical simulations using a statistically derived boundary condition model. This new method for establishing boundary conditions allows users to include event-specific observations associated with a dynamic plasma sheet. It is found that spatial and energy distributions in the storm-time ring current exhibit sensitive dependence on boundary conditions during these events. The coupling of boundary conditions to the time history of the convection electric field strength is found to play an important role in throttling the influence of the boundary plasma on the inner magnetosphere. Storm-time dusk-dawn asymmetries consistent with observational data are reproduced well when CRCM is provided with the event-specific boundary condition model. The dependence of average, global magnetospheric ion temperatures derived from ENA maps is also investigated as a function of various combinations of solar wind parameters, IMF parameters, and geomagnetic indices. Covering a 31-month interval of time near solar maximum, the parametric study reveals average stormtime features consistent with various in situ observations, ionospheric observations, and ground-based measurements. 


\section{Acknowledgment}

I would like to acknowledge the greater plasma physics community at West Virginia University for contributing to such a rich, well-developed learning environment. I'm thankful for being provided such a unique, excellent sense of continuity and completeness in our pursuit of educating one another. I owe special consideration to Dr. Amy Keesee, my Ph.D. advisor, through whose support and direction I have been provided a wealth of enriching learning opportunities. I would also like to specifically thank Dr. Earl Scime and Dr. Paul Cassak for our enlightening discussions over the recent 4.5 years. The three of you are phenomenal mentors. Numerous colleagues also deserve thanks for taking the time to assist in training our research group to use the necessary tools. Specifically, Dr. Mei-Ching Fok, Dr. Natalia Buzulukova, and the TWINS Science Team.

I would like to also acknowledge a small subset of the people who have helped shape me as a scientist and creative thinker. Specifically, Dr. Chuck Crawford, Dr. Hang Deng-Luzader, Dr. Kurt Hoffman, Mrs. Abigail LePage, Dr. Stephen Luzader, Dr. George Plitnik, Mr. Don Robinson, Mrs. Laura Sharp-Wilson and Dr. H. Arthur Weldon. Your dedication to educating others is empowering. Thank you.

I would also like to acknowledge fellow WVU graduate students and colleagues with whom I have shared myriad discussions on all imaginable topics; in particular, Matthew Beidler, Jerry Carr, Justin Ellis, Matthew Galante, Colin Komar, Mike Lindon, Rich Magee, John McKee, Stephanie Sears, and Jeffrey Walker. 
To my mother, and to my grandmother Jean. Without you, this dedication could not be written. You have believed in me without condition. 


\section{Contents}

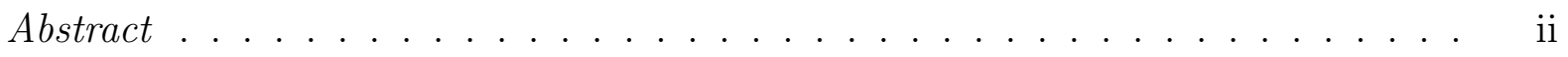

Acknowledgements ........................... ii

Dedication .......................... iv

Table of Contents............................... v

List of Figures . . . . . . . . . . . . . . . . . . . . . viii

1 Motivation 1

1.1 System Overview: The Sun-Earth Connection . . . . . . . . . . . . . . . . 1

1.1.1 Plasma and fields in the solar wind . . . . . . . . . . . . 2

1.1.2 Origin, Formation, and Structure of Earth's Magnetosphere . . . . 4

1.1.3 Sources and Characteristics of Magnetospheric Plasma . . . . . . . 7

1.2 Scope of Current Research . . . . . . . . . . . . . . . . . . . . 11

1.3 Research Goals . . . . . . . . . . . . . . . . . . . . . . . 15

References . . . . . . . . . . . . . . . . . . . . 18

2 Tools and Methods $\quad 25$

2.1 Inner Magnetospheric Numerical Models . . . . . . . . . . . . . . . . 25

2.2 Computations used for CRCM Data Analysis . . . . . . . . . . . 33 
2.3 Spacecraft Observations . . . . . . . . . . . . . 37

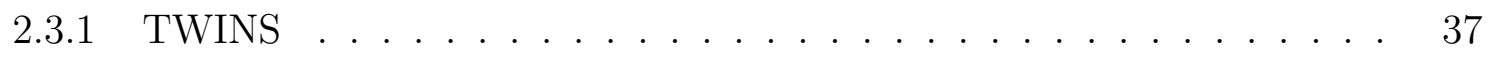

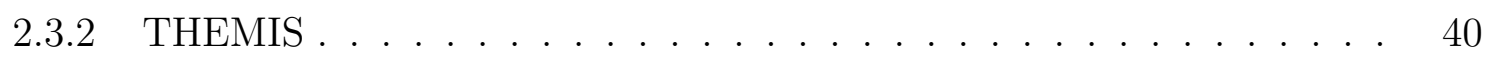

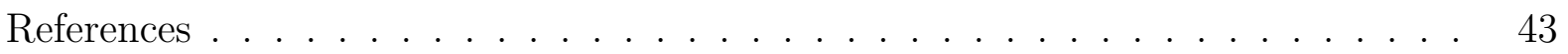

3 Implementation of Boundary Conditions 49

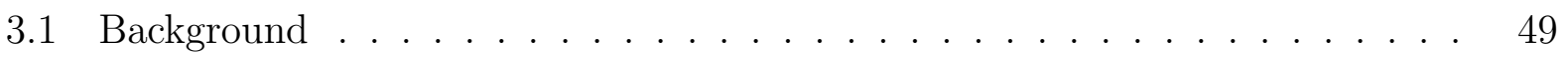

3.2 Empirical Boundary Conditions in Space Plasma Simulations . . . . . . . . . 51

3.2.1 Borovsky Model . . . . . . . . . . . . . . . . 53

3.2.2 Tsyganenko \& Mukai Model . . . . . . . . . . . . . . . 55

3.2.3 Ionospheric Measurements . . . . . . . . . . . . . . . 60

3.3 Determination of Ion Temperature Boundary Conditions from ENA maps . . 61

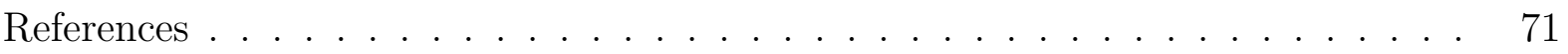

$\begin{array}{lll}4 & \text { Event Studies } & 79\end{array}$

4.1 The 22 July 2009 Event . . . . . . . . . . . . . . . . . . 80

4.1.1 Solar Wind Parameters . . . . . . . . . . . . . . . 80

4.1.2 Storm-time Ion Temperatures . . . . . . . . . . . . . . 83

4.1 .3 Simulation Results . . . . . . . . . . . . . . 93

4.1 .4 Data-Model Comparisons . . . . . . . . . . . . . 101

4.2 The $30-31$ October 2013 Event . . . . . . . . . . . . . . . . . . . 114

4.2.1 Solar Wind Parameters . . . . . . . . . . . . . . . . . 114

4.2.2 Storm-time Ion Temperatures . . . . . . . . . . . . . 115

4.2 .3 Simulation Results . . . . . . . . . . . . . . . . . 123

4.2.4 Data-Model Comparisons . . . . . . . . . . . . . . 128 
4.3 Event Study Comparison . . . . . . . . . . . . . . . . 139

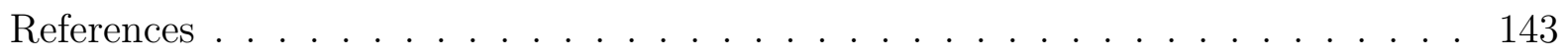

5 Parametric Analysis of Magnetospheric Ion Temperatures 149

5.1 TWINS Binning Procedure and the ACE Data Set . . . . . . . . . . 151

5.2 Single-Parameter Dependences . . . . . . . . . . . . . . . . . 152

5.2.1 Dst Dependence . . . . . . . . . . . . . . . 153

5.2.2 Solar Wind Pressure Dependence . . . . . . . . . . . . . . 156

$5.2 .3 \quad \mathrm{IMF} B_{z}$ Dependence . . . . . . . . . . . . . . . 158

5.3 Multi-Parameter Dependences ................... 160

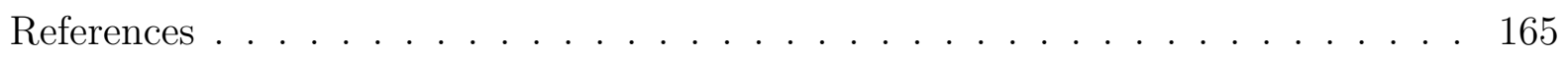

6 Conclusions $\quad 167$

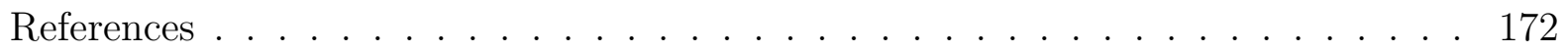




\section{List of Figures}

1.1 Overview diagram of the Sun-Earth connection . . . . . . . . . . . . 2

1.2 Illustration of the Parker spiral . . . . . . . . . . . . . . . . . 4

1.3 Diagram of important regions in Earth's magnetosphere . . . . . . . . . 5

1.4 Illustration of the physical features in a CIR . . . . . . . . . . . . . 10

2.1 Flow chart of the Vasyliunas loop . . . . . . . . . . . . . . . . . 27

2.2 Flow chart of the RCM loop . . . . . . . . . . . . . . . . . 28

2.3 The TWINS instrument and operation diagram . . . . . . . . . . . . 38

2.4 Illustration of TWINS 1 and 2 orbits . . . . . . . . . . . . . . . 39

3.1 Comparison of Maxwellian and kappa distributions . . . . . . . . . . . 53

3.2 Geotail coverage maps for the TM empirical model . . . . . . . . . . . 56

3.3 Comparison of temperatures from Borovsky model and TM model . . . . . . 59

3.4 Illustration of line-of-sight (LOS) TWINS measurements . . . . . . . . . 67

3.5 Illustration of FOV projection method . . . . . . . . . . . . . . 68

4.1 Solar wind parameters and geomagnetic indices for 22 Jul 2009 . . . . . . . . 81

4.2 ENA-derived equatorial ion temperature maps for 0000-0126 UT 22 Jul 200984

4.3 ENA-derived equatorial ion temperature maps for 0155-0321 UT 22 Jul 200985 
4.4 ENA-derived equatorial ion temperature maps for 0350-0516 UT 22 Jul 200986

4.5 ENA-derived equatorial ion temperature maps for 0759-0925 UT 22 Jul $2009 \quad 87$

4.6 ENA-derived equatorial ion temperature maps for 1051-1436 UT 22 Jul 200989

4.7 ENA-derived equatorial ion temperature maps for 1602-2326 UT 22 Jul 200990

4.8 Ion temperature boundary condition comparison for 22 Jul 2009 event . . . . 92

4.9 Sym-H* comparison for 22 Jul $2009 \ldots \ldots$. . . . . . . . . . . . . . 94

4.10 Drift Paths for 22 Jul 2009 event . . . . . . . . . . . . . . . 95

4.11 Simulated pressure during the 22 Jul 2009 event . . . . . . . . . . . . . . 97

$4.1212 \mathrm{keV}$ fluxes during the 22 Jul 2009 event . . . . . . . . . . . . . . . 99

$4.1328 \mathrm{keV}$ fluxes during the 22 Jul 2009 event . . . . . . . . . . . . . . 100

4.14 Flux vs MLT and energy at geosynchronous orbit for 22 Jul 2009 . . . . . . 102

4.15 Ion temperature comparison at geosynchronous orbit for 22 Jul 2009 . . . . 103

4.16 Orbit of THEMIS-D during the 22 Jul 2009 event . . . . . . . . . . . . 105

4.17 THEMIS-D spectrograms and temperature moments for 22 Jul 2009 . . . . 106

4.18 THEMIS-D energy spectra during 22 Jul 2009 . . . . . . . . . . . . . 107

4.19 THEMIS-E orbit 22 Jul $2009 \ldots \ldots$. . . . . . . . . . . . 111

4.20 THEMIS-E spectrograms and temperature moments . . . . . . . . . . . . 112

4.21 OMNIWeb data for the 30-31 Oct 2013 event . . . . . . . . . . . . 115

4.22 ENA-derived equatorial ion temperature maps for 0224-0540 UT 30 Oct 2013117

4.23 ENA-derived equatorial ion temperature maps for 0740-1136 UT 30 Oct 2013118

4.24 ENA-derived equatorial ion temperature maps for 1200-1559 UT 30 Oct 2013119

4.25 ENA-derived equatorial ion temperature maps for 1738-2049 UT 30 Oct 2013120

4.26 ENA-derived equatorial ion temperature maps for 2334-0735 UT 30-31 Oct

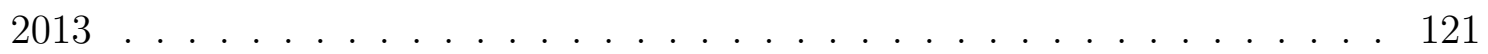


4.27 Ion temperature boundary condition comparison for 30-31 Oct 2013 event . . 122

4.28 Simulated pressure during the $30-31$ Oct 2013 event . . . . . . . . . . . . . . 124

4.29 Simulated $12 \mathrm{keV}$ flux during the $30-31$ Oct 2013 event . . . . . . . . . . . . 125

4.30 Simulated $28 \mathrm{keV}$ flux during the $30-31$ Oct 2013 event . . . . . . . . . . . . 126

4.31 Flux vs MLT and energy at geosynchronous orbit for 30-31 Oct 2013 . . . 128

4.32 Ion temperature comparison at geosynchronous orbit for 30-31 Oct 2013 . . 129

4.33 Orbit of THEMIS-A during the 30-31 Oct 2013 event . . . . . . . . . . . . . 131

4.34 Orbit of THEMIS-D during the $30-31$ Oct 2013 event . . . . . . . . . . . . . 132

4.35 Comparison of observed and simulated THEMIS-A spectrograms during 30-31

Oct $2013 \ldots \ldots \ldots \ldots \ldots$

4.36 Comparison of observed and simulated THEMIS-D spectrograms during 30-31

Oct $2013 \ldots \ldots \ldots \ldots \ldots \ldots \ldots$

4.37 Average ion temperatures from Keesee et al. . . . . . . . . . . . . . . . . . . 140

4.38 Comparison of CME and HSS simulations and observations . . . . . . . . . 141

5.1 Sunspot number for solar cycles 23-24 . . . . . . . . . . . . . . . . 150

5.2 Ion temperature dependence on Dst . . . . . . . . . . . . . . . . . . . 154

5.3 Ion temperature dependence on solar wind pressure . . . . . . . . . . . . 157

5.4 Ion temperature dependence on $\operatorname{IMF} B_{z} \ldots \ldots \ldots \ldots$

5.5 Ion temperature dependence on $B_{z}$ for high solar wind pressure $\ldots$. . . . . 162

5.6 Ion temperature dependence on $B_{z}$ for low solar wind pressure . . . . . . . 163 


\section{Chapter 1}

\section{Motivation}

\subsection{System Overview: The Sun-Earth Connection}

Planetary magnetospheres are inherently complex and dynamic physical structures, primarily due to intrinsic coupling with the planet's host star. The characteristics of the host star determine the plasma environment in which a magnetosphere is immersed and thus ultimately control characteristic processes and dynamics within the planet's magnetosphere. In the case of our solar system the host star is, of course, the Sun. Most of the planets in our solar system are magnetized: Mercury, Earth, Mars, Jupiter, Saturn, Uranus, and Neptune have all been found to be magnetized to varying degrees. In general, the character and source of the planetary fields are different because the planetary chemistry, core structure, physical history, and magnetic field source of the planets are unique, among a host of other contributing factors; see Kallenrode ${ }^{1}$ and Kivelson ${ }^{2}$ for overviews of the planetary magnetospheres in our solar system. Generally speaking, the plasma and magnetic field sourced from the Sun drive dynamics within planetary magnetospheres, and the magnetospheric plasma and fields respond through reconfigurations and transport processes in order to counteract 
the disturbances. A simplified artistic depiction of the Sun-Earth connection through interplanetary space is shown in Figure 1.1, which is courtesy of NASA; this figure shows a two-dimensional (2-D) north-south cut in the Sun-Earth plane. White lines emanating from the Sun's surface roughly indicate the solar wind plasma flow direction, the cyan lines indicate Earth's magnetic field lines, and the purple region corresponds to the standing shock which effectively separates the solar wind from Earth's magnetosphere. Note that distances are not to scale in this image.

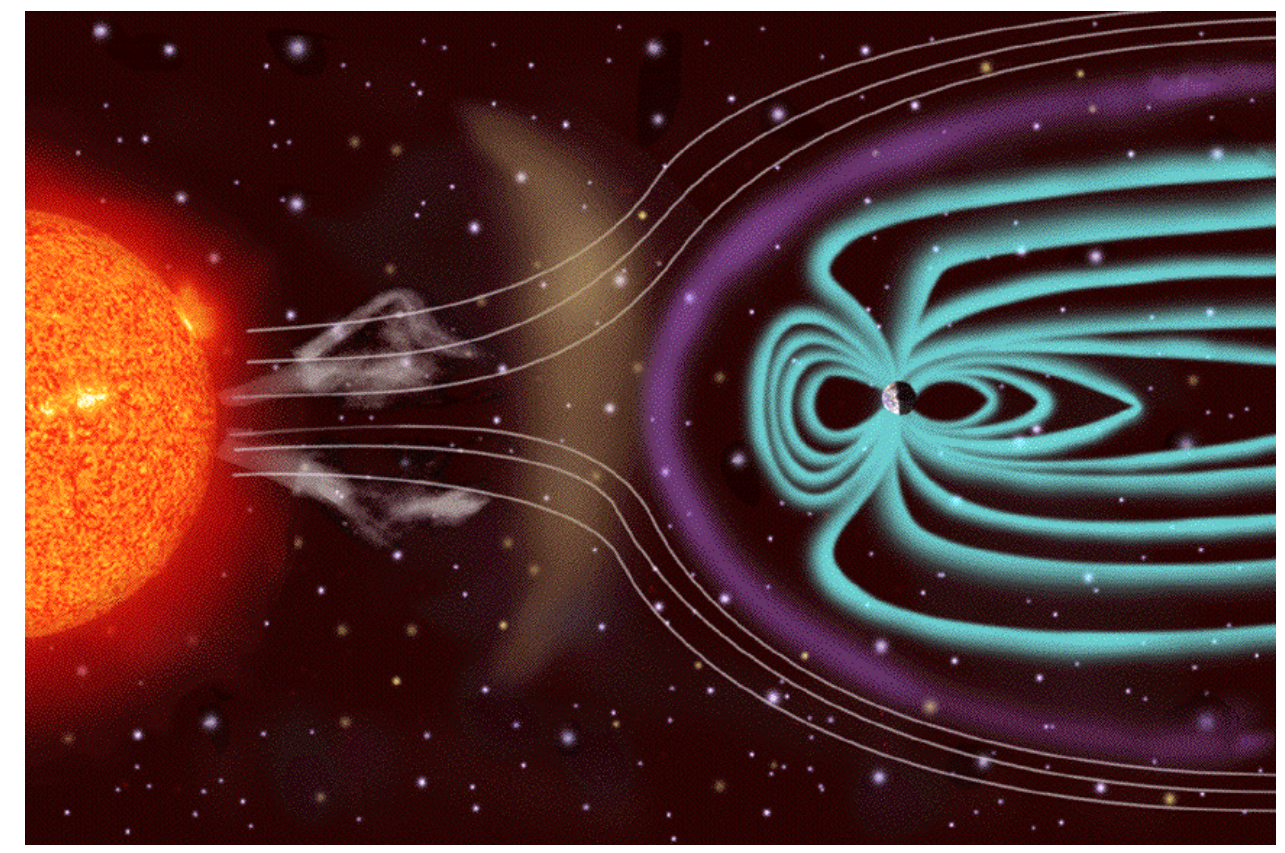

Figure 1.1: Cartoon diagram (not to scale) illustrating the Sun-Earth connection. Image courtesy of NASA.

\subsubsection{Plasma and fields in the solar wind}

There exists a net radially outward flux of plasma and magnetic field emitted from the Sun's surface. The solar atmosphere is abruptly heated across the transition region separating the chromosphere and corona; coronal temperatures are $\sim 1$ million Kelvin $(\sim 100 \mathrm{eV})$, whereas 
chromospheric temperatures are 25,000 K. The hot coronal plasma is emitted from the solar atmosphere as the solar wind and travels through interplanetary space. ${ }^{3}$ On the Sun's surface, highly complex magnetic field topologies are present. The field structure is a superposition of the internal dipole field of the Sun and the magnetic field associated with convective flow and turbulent plasma dynamics. Here the magnetic field is coupled to the conducting plasma in the sense that Alfven ${ }^{4}$ described, i.e., as the plasma and field leave the Sun's surface, the interplanetary magnetic field (IMF) remains effectively 'frozen' to the solar wind plasma.

The solar wind is a neutral plasma with a typical bulk flow velocity of 300-500 km/sec; typical quiet-time ion and electron densities are in the 1-10 particles per $\mathrm{cm}^{3}$ range, while proton temperatures are typically $\sim 10 \mathrm{eV}$, and electron temperatures are a few $\mathrm{eV} .{ }^{1}$ Protons and alpha particles are the primary ion constituents of the solar wind plasma, but other trace ion species are present due to the Sun's makeup and chemistry. Hydrogen $\left(\mathrm{H}^{+}\right)$and helium $\left(\mathrm{He}^{++}\right)$typically constitute $90-95 \%$ and $5-10 \%$ of the solar wind ions, respectively, with a $\mathrm{He}^{++} / \mathrm{H}^{+}$temperature ratio of four (see Bame et al. ${ }^{5}$ and references therein). The character of the solar wind and IMF is a strong function of the solar cycle phase, which alternates between successive solar maxima or minima every 11 years on average. In addition to temporal variations due to solar cycles, the solid-body rotation of the Sun introduces additional temporal effects. The rotation period is 27 days at the solar equator, with decreasing rotation speed going poleward in either direction. The most important effect is that since the plasma and field are frozen together and emitted radially outward from the surface, the Sun's rotation causes the flow lines to spin up and form an Archimedian spiral shape in the equatorial plane. This well-known theoretical result, originally detailed by Parker ${ }^{6}$ is commonly called the 'Parker spiral'; Figure 1.2 shows an example snapshot of plasma density and velocity on 08 May 2014, where the spiral signatures are clearly visible. Panels on the left of Figure 
1.2 are Sun-centered (yellow dot) with Earth (green dot) and STEREO (blue, red) shown at $1 \mathrm{AU}$, and show data in Earth's orbital plane. The middle panels illustrate density and radial velocity data in the north-south plane, and the right panels show the time evolution of both parameters. Figure 1.2 was generated through the NOAA WSA-Enlil Space Weather Prediction Center (SWPC) interface.

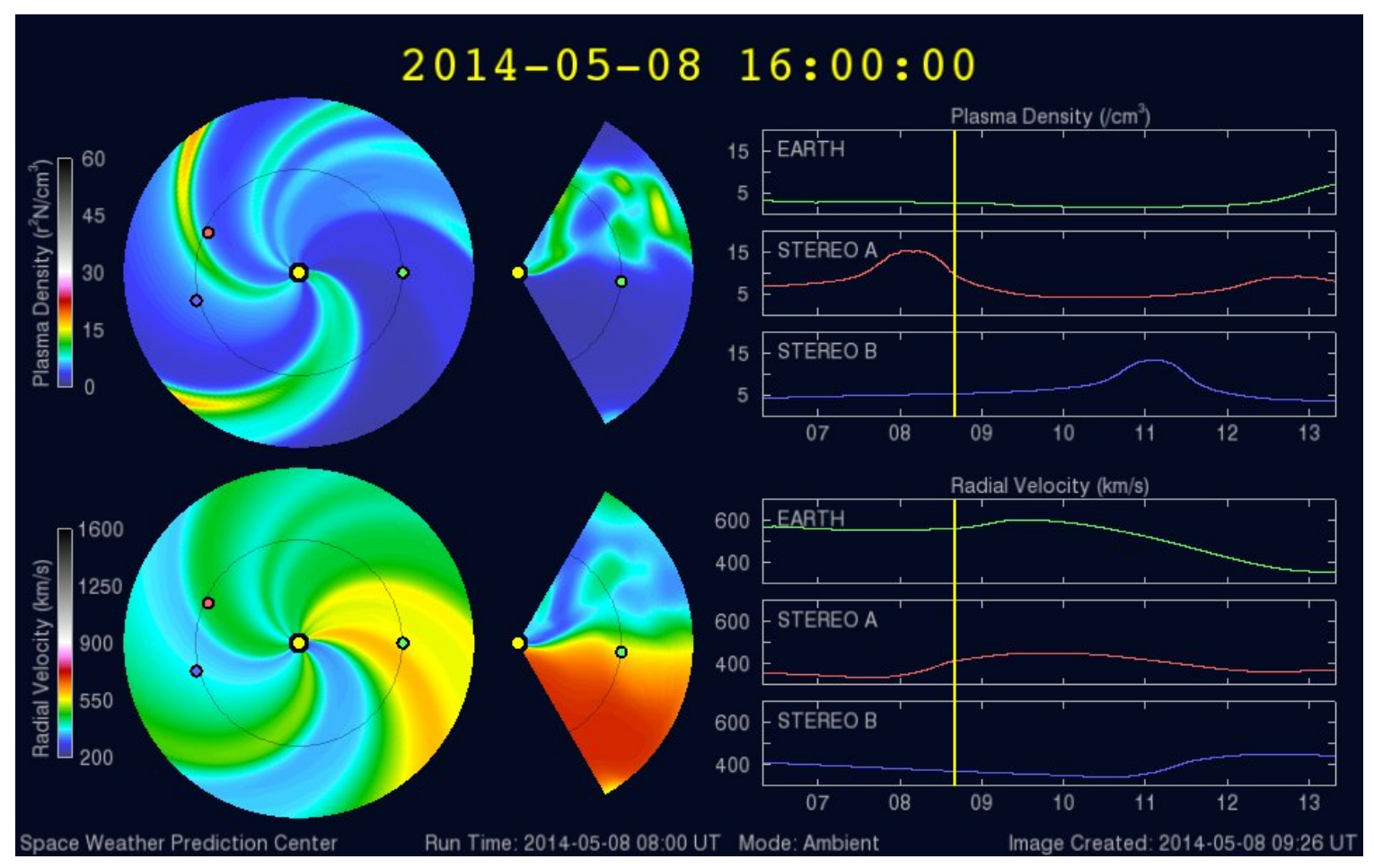

Figure 1.2: An example of the Parker spiral in the solar wind for May 8, 2014. Figure provided through the NOAA WSA-Enlil Solar Wind Prediction Service.

\subsubsection{Origin, Formation, and Structure of Earth's Magnetosphere}

Earth's internal magnetic field serves as an obstacle to the supersonic solar wind flow. Solar wind plasma is shocked as it is diverted around the Earth and its magnetic field (in three dimensions), resulting in the standing shock wave called the Bow Shock (purple region in Figure 1.1). The sheath region comprised of shocked plasma is called the magnetosheath, and 


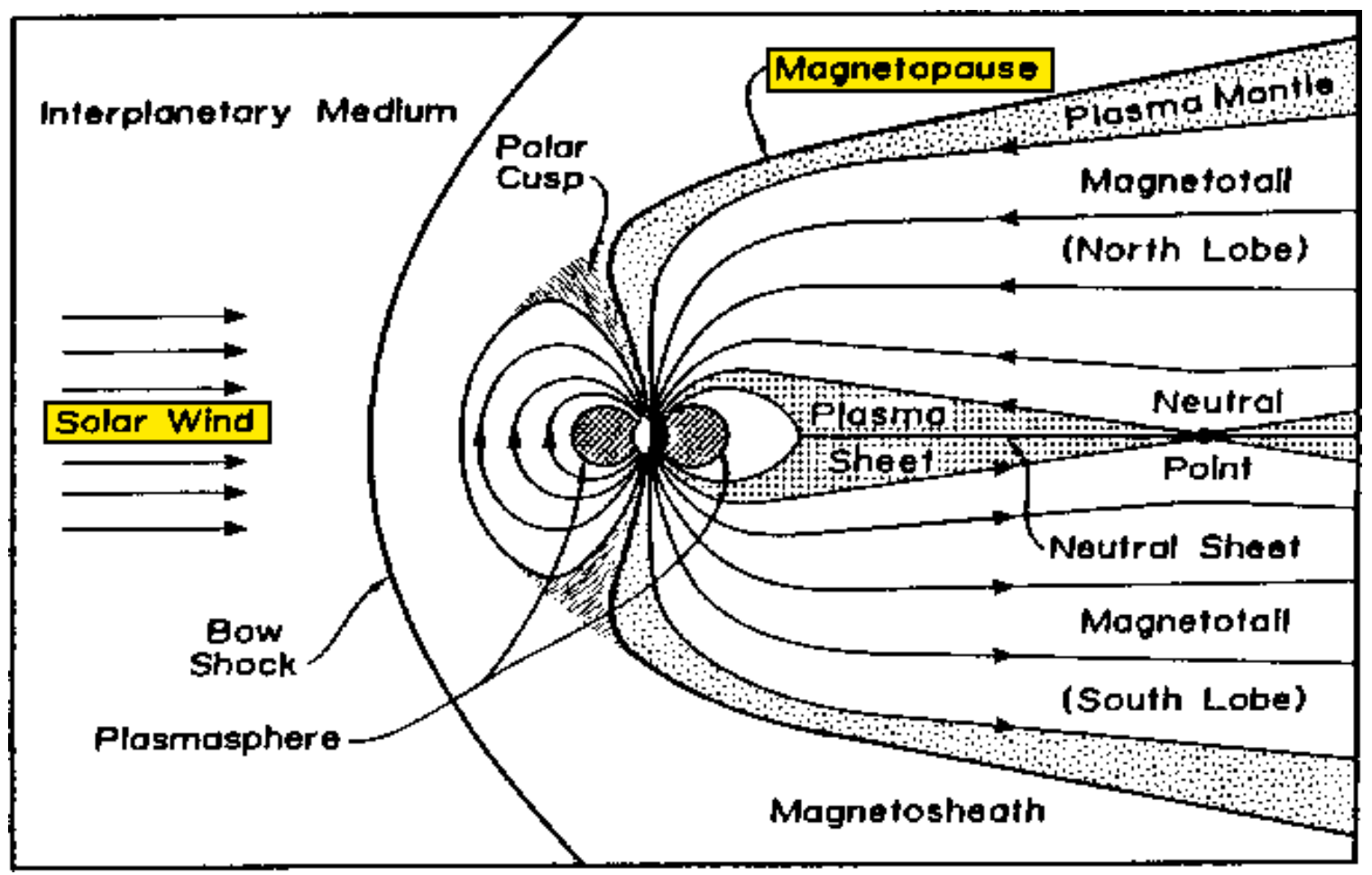

Figure 1.3: Simplified diagram of a slice of Earth's magnetosphere along the noonmidnight meridian. Figure courtesy of Russell. ${ }^{7}$

the inner terminus of the magnetosheath is the called the magnetopause. The magnetosphere is commonly regarded as the 3-D cavity bound between the magnetopause and the Earth's atmosphere. A noon-midnight meridional 2-D illustration of the relative placement of these regions is shown in Figure 1.3, where the Sun is to the left. Figure 1.3 is taken from Russell. ${ }^{7}$ Note the location of the plasma sheet, which occupies the nightside region outside of the last closed magnetic field line.

The time-dependent structure of the idealized 2-D magnetopause sheet is determined through a simple balance of pressure on either side of the boundary. This balance determines the magnetopause shape, and thus contributes significantly to the topology and general shape of the magnetosphere as a whole. In the solar wind, the total pressure is obtained as the sum of the gas pressure $n k_{B} T$, magnetic pressure $B^{2} / 8 \pi$, and ram pressure $P_{\text {ram }}$. There is 
no significant ram pressure component in Earth's magnetosphere so only the magnetic and gas pressure need be taken into account. Thus, pressure balance is obtained through the relation

$$
\left(B^{2} / 8 \pi+\sum_{\sigma} n_{\sigma} k_{B} T_{\sigma}+P_{\mathrm{ram}}\right)_{\mathrm{SW}}=\left(B^{2} / 8 \pi+\sum_{\sigma} n_{\sigma} k_{B} T_{\sigma}\right)_{\mathrm{MS}}
$$

where the sums are over each species $\sigma$. The subscript SW stands for 'solar wind', and MS stands for 'magnetosphere'.

Earth has an internal, primarily dipolar magnetic field with a dipole moment of $31.2 \mu \mathrm{T}$ - $R_{E}^{3}$. Here, $1 \mu \mathrm{T}=10^{-6}$ Tesla and $1 R_{E}=1$ Earth radius $\approx 6370 \mathrm{~km}$. The magnetic field is known to arise from a dynamo process, although such a description is inadequate when attempting to completely describe the structure of the ground magnetic field, because localized depressions and perturbations are prevalent. ${ }^{8}$ Nevertheless, Earth's field is welldescribed as a dipole field for low latitudes. The solar wind exerts a significant influence on the magnetic field morphology throughout Earth's magnetosphere, and these external field contributions are superimposed onto the dipole field. The external components arise from the diamagnetic response of various magnetospheric current systems to the persistent, dynamic solar wind forcing. A significant body of research has been dedicated to understanding the global configuration of the external field components. An exact description of the global field and magnetopause topology cannot be obtained without approximations or assumptions because of strong coupling between magnetospheric regions and the fundamentally timedependent nature of the entire system. ${ }^{9}$

The early analytical model by Olson and Pfitzer ${ }^{10}$ treated the quiet-time magnetospheric magnetic field as such a superposition by including external field contributions from the mag- 
netopause current system and quiet-time ring current. In this work the authors used a power series formulation, following the work of Carl Gauss. Tsyganenko and Usmanov ${ }^{11}$ improved pre-existing quantitative models of the magnetotail region by including representation of the downtail magnetopause current system; this model was based on in situ measurements. Later, the series of successive improvements published by Tsyganenko et al. (see publications from 1987 through 2004) revolutionized the research field and the analytical methods described by Tsyganenko et al. have proven to agree increasingly well with space-based and ground-based measurements; use of the Tsyganenko magnetic field models is ubiquitous in

space plasma physics research. The archetypical near-Earth field contributions produced by the Tsyganenko field models can be seen in Figure 1.3. The dipolar field on the dayside is compressed under the dynamic pressure of the solar wind. On the nightside, the field is stretched into the magnetotail configuration; this stretching results from tangential stresses on the magnetopause. ${ }^{12}$ The time dependent tilt of Earth's dipole field relative to the orbital plane is ignored in the creation of Figure 1.3.

\subsubsection{Sources and Characteristics of Magnetospheric Plasma}

The Dungey cycle, originally described by Dungey, ${ }^{14}$ is widely accepted to be the most important mechanism through which solar wind energy is transferred into Earth's magnetosphere. ${ }^{13}$ As discussed in the preceding section, the solar wind plasma is primarily electrons, protons, and alpha particles. During a southward-oriented IMF (negative $B_{z}$ in most coordinate systems), which is opposite in direction to Earth's northward-oriented dipolar field, magnetic reconnection may occur at the subsolar point or at variable locations along the magnetopause. After such a dayside reconnection event, the field tension is released and the reconnected field lines transport plasma tailward along the outer edge of the magnetosphere. 
Solar wind plasma and magnetic flux are transported into the magnetotail by deposition into the plasma mantle and lobes. The increased plasma and magnetic field pressure results in a highly stretched magnetotail, which given some perturbation may produce a local X-line, also called a magnetic null or neutral point. This unstable magnetic configuration results in magnetotail reconnection, typically at distances centered at $20 R_{E}$ near the midnight meridian, which ultimately feeds the night-side ion and electron plasma sheets due to the Earthward outflow jet. ${ }^{15}$ The Earthward reconnection exhaust causes bursty injections of fast, hot plasma into the inner magnetosphere, which are known to be associated with substorm activity, bursty bulk flows, and additional impulsive processes. ${ }^{16,17}$ See Nishida ${ }^{18}$ for a compact review on the importance of magnetic reconnection in Earth's magnetosphere. During periods of enhanced convection, which directly correspond to enhanced geomagnetic activity levels, the plasma sheet is the primary source for feeding fresh electrons and ions into the inner magnetosphere. The plasma sheet is a highly dynamic structure; the shape and characteristic plasma parameters depend on solar wind conditions, magnetic field topology, and are known to correlate with various geomagnetic indices (see Section 1.2). Under average conditions, the inner edge of the plasma sheet is located in the 8-12 $R_{E}$ range on the midnight meridian.

The Earth itself is being increasingly acknowledged as an important source of magnetospheric plasma. ${ }^{19,20}$ During periods of enhanced geomagnetic activity, ions of ionospheric origin (primarily $\mathrm{O}^{+}$) upwell into the magnetosphere due to enhanced ambipolar electric fields in the upper ionosphere. Ionospheric upwelling is weak during low-activity periods, but can source very significant $\mathrm{O}^{+}$fluxes during intervals of heightened geomagnetic activity. Young et al. ${ }^{21}$ found that the storm-time $\mathrm{O}^{+} / \mathrm{H}^{+}$ratio can exceed $50 \%$ during highly active periods occurring near solar maximum. Daglis ${ }^{22}$ confirmed that the ring current may be 
dominated by $\mathrm{O}^{+}$ions during the most intense periods of the storm; the ring current is the principle indicator of geomagnetic disturbances, and is discussed in greater detail later in this chapter.

The fundamentally time dependent nature of solar activity is reflected in solar wind plasma parameters (e.g., speed, density, temperature, ion composition). In addition to regular variations, such as the 11 year solar cycle, transient features in the solar wind associated with large-scale disturbances are also present. The two most important types of disturbance are highly geoeffective, and frequently result in intense space weather activity. Geoeffectiveness refers to how efficiently solar wind energy can be transported or otherwise converted to energy within the magnetosphere. ${ }^{23}$

A coronal mass ejection (CME) is driven by magnetic reconnection in the solar atmosphere. Magnetic energy stored in the surface magnetic fields is abruptly converted to bulk kinetic energy and thermal energy of solar plasma ejected into interplanetary space. These ejecta can have average mass of around $10^{12} \mathrm{~kg}$, move through space at speeds well above $1000 \mathrm{~km} / \mathrm{s}$, and have average kinetic energies exceeding $10^{23}$ Joules. ${ }^{24}$ Intense magnetic fields are frequently associated with these structures; 20-30 nT IMF magnitudes are common, and are occasionally found to be as high as $60 \mathrm{nT}$. These strong fields can be extremely efficient in transferring solar wind plasma into Earth's magnetosphere.

A corotating interaction region (CIR) is formed when fast solar wind streams, which emanate from coronal holes, interact with the slower, upstream solar wind. This interaction results in a local compression of the plasma and magnetic field; the compression region is called a CIR. A diagram illustrating the formation of a CIR is shown in Figure 1.4. The magnetospheric response to a high-speed stream (HSS) is considerably different than for CME-driven events. Most notably, CIR-driven geomagnetic storms have a much longer 
duration and Earth's magnetosphere can remain in a disturbed state for days or weeks. In addition, CIR-driven events usually have a comparatively low to moderate maximum instantaneous intensity, but significant amounts of energy are transferred to the magnetosphere because of the long duration; in fact, CIR-driven storms can result in more efficient energy coupling between the solar wind and magnetosphere than for CME-driven events. ${ }^{25}$
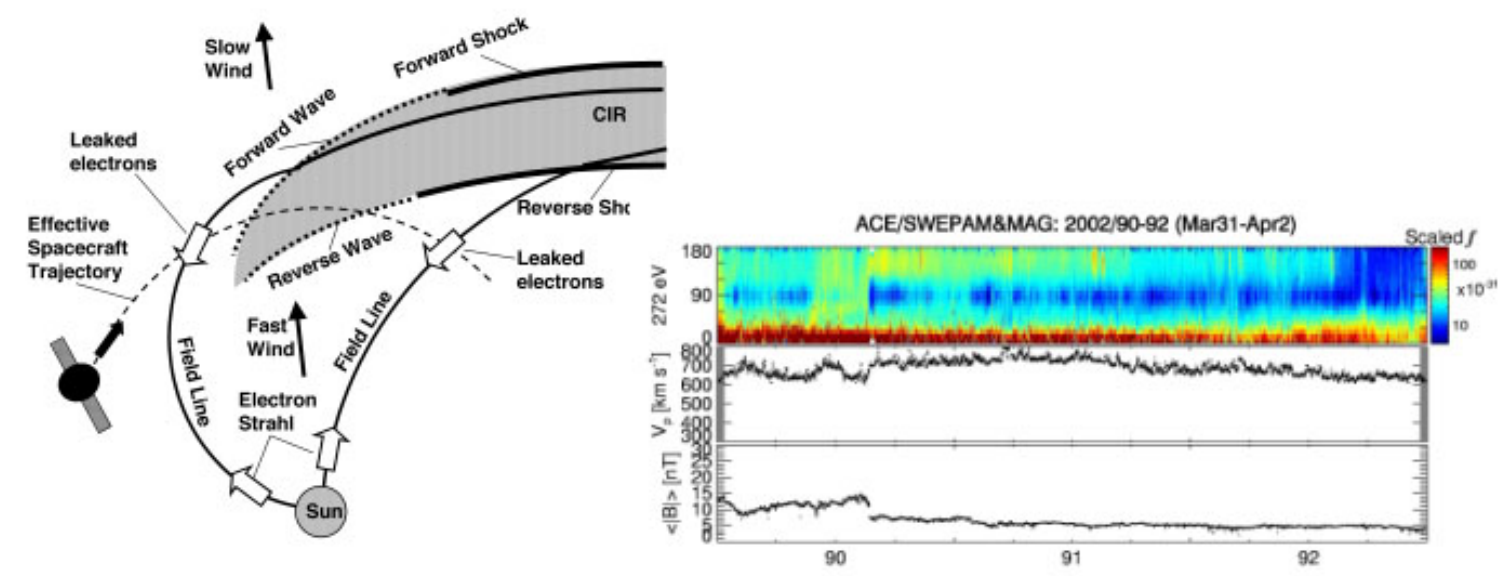

Figure 1.4: Diagram illustrating the structure and formation of a CIR through the interaction of fast and slow solar wind streams. From Steinberg et al. ${ }^{26}$

The highly variable character of plasma entering the storm-time inner magnetosphere results in a rather complex picture inside of the inner plasma sheet edge. A well-established metric used to indicate the intensity of geomagnetic activity is called the 'Disturbance storm time' index (Dst index), which is highly correlated with the strength of the ring current. The ring current is a roughly toroidal, westward-directed current system encircling the Earth and spanning from $\sim 2-9 R_{E}$; the magnetic field perturbations associated with the ring current are diamagnetic and thus decrease the nominal ground magnetic field as the ring current intensity increases. The Dst index is a measure of the magnetic disturbance, typically given in $\mathrm{nT}\left(10^{-9}\right.$ Tesla), and is directly related to the total ring current energy. ${ }^{27}$ This current system is a kinetic region that is highly dynamic in space and time, reflecting conditions in 
the solar wind and elsewhere in the magnetosphere. The ring current is composed of ions and electrons; the ions originate in the plasma sheet and the ionosphere, as previously discussed. Electrons do not contribute significantly to the ring current, because of their low energy density and fast loss timescales. Ring current ions generally have energies of a few $\mathrm{keV}$ up to hundreds of keV. During moderate magnetic storms, the Dst index can drop as low as -100 $\mathrm{nT}$, whereas during severe, typically CME-driven storms the Dst can fall below -300 nT. Geomagnetic storms of this magnitude (-300 nT) are somewhat rare, occurring only a few times per solar cycle. See Daglis et al. ${ }^{20}$ for a thorough exposition on the ring current, its storm-time morphology and ion composition, and its broader role in magnetospheric physics.

\subsection{Scope of Current Research}

As emphasized in Section 1.1, the plasma sheet is an important source of inner magnetospheric plasma, especially during periods of enhanced geomagnetic activity. Many authors have studied the connection between the state of the plasma sheet and the solar wind conditions, i.e., how the density, temperature, and pressure of the plasma sheet correlate with the state of the solar wind; see e.g., Baumjohann et al. ${ }^{28}$ Borovsky et al. ${ }^{29}$ Tsyganenko and Mukai, ${ }^{30}$ and Wing and Newell. ${ }^{31,32}$ Spacecraft observations indicate that the plasma sheet tends to be cold and dense during periods of prolonged northward IMF. ${ }^{33-35}$ When the IMF is southward, magnetic reconnection controls loading and unloading of the plasma sheet through the Dungey cycle, and during prolonged southward periods the plasma sheet becomes hotter and less dense as geomagnetic activity increases. ${ }^{32,33}$ In reality these prolonged, steady-state configurations are idealized representations of Earth's plasma sheet, intentionally studied to shed light on simplified physical connections between the solar wind, 
the plasma sheet, and the inner magnetosphere. It is well known that the solar wind-driven plasma sheet is highly dynamic during storm time, and also that plasma sheet parameters exhibit strong dependence on geomagnetic activity levels. Baumjohann et al. ${ }^{28}$ characterized the average dependence of central plasma sheet ion temperatures on activity levels and reported that ion temperatures during active periods increase by a factor of 3-5 over those found during quiet periods. Wang et al. ${ }^{36}$ performed a statistical analysis using Geotail data to understand how the nightside plasma sheet structure varies under the influence of different IMF $B_{z}$ conditions. The $B_{z}$ orientation and duration is known to be the main driver of storms, and they found that hotter nightside ion temperatures occur for shorter (longer) intervals of sustained northward (southward) $B_{z}$. The authors correlated dusk-dawn asymmetries with expectations from drift theory and also found that active-time plasma sheet ion temperatures increase by a factor of roughly 3-5 compared to low-activity intervals.

The spatial structure of the plasma sheet may also change on variable timescales due to substorm activity, magnetic reconnection, or magnetic field dipolarizations. ${ }^{37,38}$ Keesee et $a l .{ }^{39}$ used energetic neutral atom observations to map the convection of a hot, localized structure during the main phase of the 22 July 2009 event. Those observations are apparently related to bursty bulk flows occurring in the substorm expansion phase. Large-scale magnetic reconfigurations resulting from, e.g., substorm activity, are responsible for the injection of magnetic flux and plasma into the inner magnetosphere and may produce, through Faraday's Law, intense inductive electric fields. In addition, the plasma sheet may exhibit dusk-dawn asymmetries in density and temperature, ${ }^{32,36,40,41}$ further complicating the nonlinear coupling between magnetospheric regions and processes.

The underlying nonlinear coupling in magnetospheric physics presents challenges, but computational models may be used to gain insights into the fundamental physics. When 
performing simulations of the inner magnetosphere, it is critical to supply accurate boundary conditions since they reflect mechanisms and processes outside of the simulation domain. Empirical models of plasma sheet particle distributions which are based on statistical averages of many in situ spacecraft measurements are commonly used to establish nightside boundary conditions. However such statistical plasma sheet models smooth out transient physics associated with phenomena including substorm processes and by-products of magnetotail reconnection. Understanding the effects caused by substorm activity or reconnection events in the storm-time magnetosphere thus requires a plasma sheet boundary condition model that is tailored to a specific time frame. For simulations of a specific event, this is ideally achieved by using temporally and spatially resolved spacecraft observations for the full simulation interval. In practice, incorporating such observations into numerical simulations will be complicated by limited spacecraft coverage. This is especially the case when using in situ measurements for boundary conditions for a specific event, because of the fundamentally local nature of those measurements. Nonetheless, others have successfully used event-specific, in situ satellite measurements as boundary conditions to inner magnetospheric models in the past. Zaharia et al. ${ }^{42}$ used LANL Magnetospheric Plasma Analyzer (MPA) and Synchronous Orbit Particle Analyzer (SOPA) data from geosynchronous orbit to supply boundary conditions for simulations of the 21-25 October 2001 event using the UNH-RAM model. ${ }^{43}$ The LANL satellites are stationed at geosynchronous orbit and thus do not provide a clear picture at 8-12 $R_{E}$ (the Earthward edge of the plasma sheet, and outer boundary in most inner magnetospheric codes), nor are MPA/SOPA data publicly available for events occurring after 2007. However, it is increasingly accepted that event-specific boundary conditions are of critical importance when performing magnetospheric simulations.

Quiet-time and storm-time computational studies of the inner magnetosphere have been 
performed in order to understand the effects of plasma sheet density and temperature on the state of the ring current. Ebihara and Ejiri ${ }^{44}$ found that storm-time ring current buildup is insensitive to constant plasma sheet ion temperatures above $3 \mathrm{keV}$. These simulations were performed using a dipole magnetic field, a Volland-Stern convection electric field ${ }^{45,46}$ coupled to the Boyle et al. ${ }^{47}$ polar cap potential, and used solar wind data provided by WIND as the model inputs. Rice Convection Model (RCM) simulations discussed by Garner ${ }^{48}$ improve on the Ebihara and Ejiri model by including the more realistic Hilmer and Voigt magnetic field model ${ }^{49}$ and a self-consistent electric field. Garner found that more intense electric shielding and stronger Region 2 currents are found when colder temperatures are provided as the plasma sheet boundary condition. Cold ion populations in the plasma sheet are dominated by the Earthward $\vec{E} \times \vec{B}$ drift since virtually no particles in the population have high enough energy to experience substantial gradient-curvature drift; see Section 2.1 for an explanation of fundamental plasma drifts. A hotter population, however, has a larger relative fraction of higher energy ions and thus fewer ions penetrate to lower $L$-shells, ${ }^{50}$ resulting in a relative decrease in pressure at low $L$ values. This results in a weaker shielding field, a weaker ring current, and a more diffuse pressure distribution in the ring current. It is reasonable to expect that including spatial variation in the ion temperature boundary conditions would modify the azimuthal and radial structure of the ring current, the global electric field, and the distribution of energy in the inner magnetosphere.

Chen et al. ${ }^{51}$ presented results from magnetically self-consistent drift-loss ring current simulations using ion temperature boundary conditions determined from time-averaged Geotail measurements for two separate cases. In both cases (a cold, dense case and a hot, tenuous case), the simulations focus on ring current preconditioning, i.e., the first few hours of the main phase of a storm, during northward IMF. The boundary conditions were held constant 
in time, but exhibited azimuthal variation and dawn-dusk asymmetry. The authors investigated the MLT dependence of the plasma sheet conditions on the formation of the storm-time ring current. They found that cold ion populations in the post-midnight quadrant produced pressure enhancements in the post-midnight sector of the ring current, and that hot, tenuous boundary conditions produced a more azimuthally-uniform pressure distribution on the night side. Thus, we expect spatio-temporal dependence in the temperature boundary conditions to play an important role when simulating the full time frame of a geomagnetic storm.

\subsection{Research Goals}

The content of this dissertation regards remotely imaged, spatiotemporally resolved magnetospheric ion temperatures. The temperatures are derived from time-resolved, 2-D maps of energetic neutral atom (ENA) fluxes in the plane of Earth's magnetic equator. First, results of Comprehensive Ring Current Model (CRCM) simulations using such ion temperatures as boundary conditions are presented and examined. Time-dependent density and temperature boundary conditions for CRCM are typically provided by a statistical plasma sheet model, but the CRCM may be easily customized to admit user-defined values. For example, another unique method for determining boundary conditions involves coupling separate magnetospheric models, such as the two-way coupled BATS-R-US + CRCM model described by Glocer et al. ${ }^{52}$ The BATS-R-US global MHD code is used to represent the global magnetosphere while CRCM is used to model the inner magnetosphere, and the two disparate models provide feedback to one another across the shared boundary. While this method improves upon a purely statistical approach, it can not incorporate event-specific inner plasma sheet observations for a given simulation time frame, which is the primary focus of our current 
research. The ion temperature boundary conditions used in the new simulations are derived using the method described by Scime et al. ${ }^{53,54}$ for the medium energy ENA instrument (MENA) on the Imager for Magnetopause-to-Aurora Global Exploration (IMAGE) mission, and later applied to ENA measurements from the TWINS mission by Keesee et al. ${ }^{39,40}$ Results from these simulations are compared to otherwise identical simulations that use ion temperatures calculated from the Tsyganenko and Mukai ${ }^{30}$ plasma sheet model. Second, ion temperatures determined from ENA maps are accumulated over a 31-month interval of time and correlated with the simultaneous solar wind and IMF parameters. Such an analysis provides a sense of the average magnetospheric response due to specific types of solar wind driving.

A description of the central tools and methods is presented in Chapter 2 of this manuscript. This includes a discussion of the computational model used to simulate Earth's inner magnetosphere, a section devoted to the computations required to perform various numerical analyses, and two sections detailing the mission specifics and instrumentation aboard the TWINS and THEMIS spacecraft. Chapter 3 contains a discussion of boundary condition models available for use in conjunction with the computational model, a discussion of the theory of determining ion temperatures through space-based charge-exchange imaging, and a discussion of the specifics of implementing such ion temperature data into the computational model's framework. Chapter 4 provides an overview and analysis of two specific geomagnetic storms. Simulation results of the 22 July 2009 and 30-31 October 2013 events are presented, with emphases on data-model comparisons and key differences observed between ion temperature boundary condition models. Chapter 5 contains a discussion of correlations between solar wind parameters and global magnetospheric ion temperatures averaged over a 31-month time frame spanning 01 Jan 2011 to 31 Jul 2013. The results from the parametric 
study contained therein illustrate the reliability of this method, as a collection of well-known results from in situ measurements and theoretical treatments are reproduced for a variety of distinct solar wind and IMF conditions. Chapter 6 contains a summary and discussion of this research. 


\section{References}

[1] M.-B. Kallenrode, Space Physics: An Introduction to Plasmas and Particles in the Heliosphere and Magnetospheres. Springer, 3rd ed., 2004.

[2] M. G. Kivelson, Handbook of the Solar-Terrestrial Environment, ch. Planetary Magnetospheres, pp. 469-492. Springer-Verlag, 2007.

[3] V. H. Hansteen, Heliophysics: Plasma Physics of the Local Cosmos, ch. The solar atmosphere, pp. 195-224. Cambridge University Press, 2009.

[4] H. Alfven, "On the existence of electromagnetic-hydrodynamic waves," Arkiv foer Matematik, Astronomi och Fysik, vol. 39, no. 2, 1943.

[5] S. J. Bame, A. J. Hundhausen, J. R. Asbridge, and I. B. Strong, "Solar wind ion composition," Physical Review Letters, vol. 20, no. 8, pp. 393-395, 1968.

[6] E. N. Parker, "The hydrodynamic theory of solar corpuscular radiation and stellar winds," The Astrophysical Journal, vol. 132, pp. 821-866, 1960.

[7] C. T. Russell, The Solar Wind and the Earth, ch. The Magnetosphere, pp. 73-100. Terra Scientific Publishing Co., 1987.

[8] U. R. Christensen, Heliophysics: Evolving Solar Activity and the climates of Space and Earth, ch. Planetary fields and dynamos, pp. 179-215. Cambridge University Press, 2010.

[9] A. T. Y. Lui, "Solar wind influence on magnetotail configuration and dynamics," in Solar Wind Magnetosphere Coupling: Proceeds of the Chapman Conference (Y. Kamide 
and J. A. Slavin, eds.), vol. 126 of Astrophysics and Space Science Library, pp. 671-690, Terra Scientific Publishing Co., 1986.

[10] W. P. Olson and K. A. Pfitzer, "A quantitative model of the magnetospheric magnetic field," Journal of Geophysical Research, vol. 79, no. 25, pp. 3739-3748, 1974.

[11] N. A. Tsyganenko and A. V. Usmanov, "Determination of the magnetospheric current system parameters and development of experimental geomagnetic field models based on data from imp and heos satellites," Planetary Space Sciences, vol. 30, p. 985, 1982.

[12] S. W. H. Cowley, Physics of the Magnetopause, vol. 90 of Geophysical Monograph Series, ch. Theoretical Perspectives of the Magnetopause: A Tutorial Review, pp. 29-43. American Geophysical Union, 1995.

[13] W. D. Gonzalez, J. A. Joselyn, Y. Kamide, H. W. Kroehl, G. Rostoker, B. T. Tsurutani, and V. M. Vasyliunas, "What is a geomagnetic storm?," Journal of Geophysical Research, vol. 99, no. A4, pp. 5771-5792, 1994.

[14] J. W. Dungey, "Interplanetary magnetic field and the auroral zones," Physical Review Letters, vol. 6, p. 47, 1961.

[15] Y. Asano, T. Mukai, M. Hoshino, Y. Saito, H. Hayakawa, and T. Nagai, "Statistical study of thin current sheet evolution around substorm onset," Journal of Geophysical Research, vol. 109, no. A5, 2004.

[16] W. Baumjohann, R. Nakamura, R. Schodel, and K. Dierschke, Disturbances in Geospace: The Storm-Substorm Relationship, vol. 142 of Geophysical Monographs, ch. Substorms, Storms, and the Storm-Time Plasma Sheet, pp. 55-58. American Geophysical Union, 2003. 
[17] V. Angelopoulos, C. F. Kennel, F. V. Coroniti, R. Pellat, M. G. Kivelson, R. J. Walker, C. T. Russell, W. Baumjohann, W. C. Feldman, and J. T. Gosling, "Statistical characteristics of bursty bulk flow events," Journal of Geophysical Research, vol. 99, no. A11, pp. 21257-21280, 1994.

[18] A. Nishida, Handbook of the Solar-Terrestrial Environment, ch. Magnetic Reonnection, pp. 279-310. Springer-Verlag, 2007.

[19] C. R. Chappell, T. E. Moore, and J. H. W. Jr., "The ionosphere as a fully adequate source of plasma for the earth's magnetosphere," Journal of Geophysical Research, vol. 92, pp. 5896-5910, 1987.

[20] I. A. Daglis, R. M. Thorne, W. Baumjohann, and S. Orsini, "The terrestrial ring current: Origin, formation, and decay," Reviews of Geophysics, vol. 37, pp. 407-438, 1999.

[21] D. T. Young, H. Balsinger, and J. Geiss, "Correlations of magnetospheric ion composition with geomagnetic and solar activity," Journal of Geophysical Research, vol. 87, no. A11, pp. 9077-9096, 1982.

[22] I. A. Daglis, Magnetic Storms, ch. The role of magnetosphere-ionosphere coupling in magnetic storm dynamics, pp. 107-116. American Geophysical Union, 1997.

[23] N. E. Turner, E. J. Mitchell, D. J. Knipp, and B. A. Emery, Recurrent Magnetic Storms: Corotating Solar Wind Streams, ch. Energetics of Magnetic Storms Driven by Corotating Interaction Regions: A Study of Geoeffectiveness, pp. 113-124. No. 167 in Geophysical Monograph Series, American Geophysical Union, 2006.

[24] P. J. Cargill and L. K. Harra, Handbook of the Solar-Terrestrial Environment, ch. Coronal Mass Ejection, pp. 117-132. Springer-Verlag, 2007. 
[25] G. Lu, Recurrent Magnetic Storms: Corotating Solar Wind Streams, ch. High-Speed Streams, Coronal Mass Ejections, and Interplanetary Shocks: A Comparative Study of Geoeffectiveness, pp. 97-111. No. 167 in Geophysical Monograph Series, American Geophysical Union, 2006.

[26] "Suprathermal electrons in high-speed streams: Counter-streaming on open field lines at 1 au," 2003.

[27] W. Baumjohann and R. A. Treumann, Basic Space Plasma Physics. Imperial College Press, 1997.

[28] W. Baumjohann, G. Paschmann, and C. A. Cattell, "Average plasma properties in the central plasma sheet," Journal of Geophysical Research, vol. 94, no. A6, pp. 6597-6606, 1989.

[29] J. E. Borovsky, M. F. Thomsen, and R. C. Elphic, "The driving of the plasma sheet by the solar wind," Journal of Geophysical Research, vol. 107, no. A8, pp. 17617-17639, 1998.

[30] N. A. Tsyganenko and T. Mukai, "Tail plasma sheet models derived from geotail particle data," Journal of Geophysical Research, vol. 108, no. A3, p. 1136, 2003.

[31] S. Wing and P. T. Newell, "Central plasma sheet ion properties as inferred from ionospheric observations," Journal of Geophysical Research, vol. 103, no. A4, pp. 6785-6800, 1998.

[32] S. Wing and P. T. Newell, "2d plasma sheet ion density and temperature profiles for northward and southward imf," Geophysical Research Letters, vol. 29, no. 9, p. 1307, 2002. 
[33] T. Terasawa, M. Fujimoto, T. Mukai, I. Shinohara, Y. Saito, T. Yamamoto, S. Machida, S. Kokubun, A. J. Lazarus, J. T. Steinberg, and R. P. Lepping, "Solar wind control of density and temperature in the near-earth plasma sheet: Wind/geotail collaboration," Geophysical Research Letters, vol. 24, no. 8, pp. 935-938, 1997.

[34] D. H. Fairfield, R. P. Lepping, E. W. Hones, S. J. Bame, and J. R. Asbridge, "Simultaneous measurements of magnetotail dynamics by imp spacecraft," Journal of Geophysical Research, vol. 86, p. 1396, 1981.

[35] W. Lennartsson, "A scenario for solar wind penetration of earth's magnetic tail based on ion composition data from the isee 1 spacecraft," Journal of Geophysical Research, vol. 97 , no. 19, p. $221,1992$.

[36] C. P. Wang, L. R. Lyons, J. M. Weygand, T. Nagai, and R. W. McEntire, "Equatorial distributions of the plasma sheet ions, their electric and magnetic drifts, and magnetic fields under different interplanetary magnetic field bz conditions," Journal of Geophysical Research, vol. 111, no. A04215, 2006.

[37] S. Ohtani, "Earthward expansion of tail current disruption: Dual-satellite study," Journal of Geophysical Research, vol. 103, pp. 6815-6825, 1998.

[38] V. A. Sergeev, D. G. Mitchell, C. T. Russell, and D. J. Williams, "Structure of the tail plasma/current sheet at 11 re and its changes in the course of a substorm," Journal of Geophysical Research, vol. 98, no. A10, pp. 17345-17365, 1993.

[39] A. M. Keesee, J. G. Elfritz, D. J. McComas, and E. E. Scime, "Inner magnetosphere convection and magnetotail structure of hot ions imaged by ena during a hss-driven storm," Journal of Geophysical Research, vol. 117, no. A00L06, pp. 1-9, 2012. 
[40] A. M. Keesee, N. Buzulukova, J. Goldstein, D. J. McComas, E. E. Scime, H. Spence, M. C. Fok, and K. Tallaksen, "Remote observations of ion temperatures in the quiet time magnetosphere," Geophysical Research Letters, vol. 38, no. L03104, pp. 1-5, 2011.

[41] Y. Zheng, A. T. Y. Lui, and M. C. Fok, "Effects of plasma sheet properties on stormtime ring current," Journal of Geophysical Research, vol. 115, no. A08220, 2010.

[42] S. Zaharia, M. F. Thomsen, J. Birn, M. H. Denton, V. K. Jordanova, and C. Z. Cheng, "Effect of storm-time plasma pressure on the magnetic field in the inner magnetosphere," Geophysical Research Letters, vol. 32, no. L03102, pp. 1-4, 2005.

[43] V. K. Jordanova, J. U. Kozyra, A. F. Nagy, and G. V. Khazanov, "Kinetic model of the ring current-atomsphere interactions," Journal of Geophysical Research, vol. 102, no. A7, pp. 14279-14291, 1997.

[44] Y. Ebihara and M. Ejiri, "Simulation study on fundamental properties of the stormtime ring current," Journal of Geophysical Research, vol. 105, no. A7, pp. 15843-15859, 2000.

[45] H. Volland, "A semiempirical model of large-scale magnetospheric electric fields," Journal of Geophysical Research, vol. 78, pp. 171-181, 1973.

[46] D. P. Stern, "Models of the earth's electric field," Tech. Rep. X-602-74-159, NASA Goddard Space Flight Center, Greenbelt, MD, 1974.

[47] C. B. Boyle, P. H. Reiff, and M. R. Hairston, "Empirical polar cap potentials," Journal of Geophysical Research, vol. 102, no. A1, pp. 111-125, 1997. 
[48] T. W. Garner, "Numerical experiments on the inner magnetospheric electric field," Journal of Geophysical Research, vol. 108, no. A10, p. 1373, 2003.

[49] R. V. Hilmer and G. H. Voigt, "A magnetospheric magnetic field model with flexible current systems driven by independent physical parameters," Journal of Geophysical Research, vol. 100, p. 5613, 1995.

[50] C. McIlwain, Earth's Magnetospheric Processes, ch. Plasma convection in the vicinity of the geosynchronous orbit, p. 268. D. Reidel Pub. Comp., 1972.

[51] M. C. Chen, C. P. Wang, M. Schulz, and L. R. Lyons, "Solar-wind influence on mlt dependence of plasma sheet conditions and their effects on storm time ring current formation," Geophysical Research Letters, vol. 34, p. L14112, 2007.

[52] A. Glocer, M. Fok, X. Meng, G. Toth, N. Buzulukova, S. Chen, and K. Lin, "Crcm + bats-r-us two-way coupling," Journal of Geophysical Research, vol. 118, pp. 1635-1650, 2013.

[53] E. E. Scime, A. M. Keesee, J. M. Jahn, J. L. Kline, C. J. Pollock, and M. Thomsen, "Remote ion temperature measurements of earth's magnetosphere: Medium energy neutral atom (mena) images," Geophysical Research Letters, vol. 29, no. 10, p. 1438, 2002.

[54] E. E. Scime and A. M. Zaniewski, "Charge exchange imaging of space plasmas," Review of Scientific Instruments, vol. 75, no. 10, pp. 3526-3530, 2004. 


\section{Chapter 2}

\section{Tools and Methods}

\subsection{Inner Magnetospheric Numerical Models}

Early studies laid the foundation for qualitative understanding of magnetospheric and ionospheric processes in relation to the solar wind. In particular, the seminal work by Axford and Hines ${ }^{1}$ outlined the basic underlying principles of magnetospheric convection and its relation to ionospheric currents and quasi-steady solar wind forcing. In conjunction with the proposed model of Dungey, ${ }^{2}$ a new era of probing magnetospheric processes was ushered in. Later, Fejer ${ }^{3}$ expanded upon earlier work by emphasizing the importance of capturing the ring current's feedback on the ionospheric system. More complete pictures of the inner magnetosphere were later discussed in a quantitative context by Vasyliunas ${ }^{4,5}$ and Wolf. $^{6}$ In these works, Vasyliunas and Wolf illustrate the typical starting point for modeling the phys-

ical processes occurring in the inner magnetosphere as a function of drivers and boundary conditions. Figure 2.1 illustrates the flow of the algorithm, adapted from Vasyliunas. The boxes in that diagram correspond to fields and plasma moments which are to be computed. The connecting lines between adjacent boxes correspond to equations which must be solved; 
the arrows on these lines illustrate the feedback direction. The red text indicates how the plasma boundary conditions and driver terms, such as the magnetic field model or equivalent current systems, couple into the algorithm. In this scenario, boundary conditions are defined either by the particle pressure at the boundary locations or, equivalently, by the boundary plasma density and temperature. Starting by prescribing initial conditions for the distribution functions and fields, the cycle moves forward in time by making a counter-clockwise loop. Assuming the magnetospheric electric and magnetic fields are known everywhere, a kinetic equation can be solved to update the distribution functions in conjunction with the prescribed boundary conditions. The kinetic equation can be based on Liouville's theorem, or can be a variation of the Boltzmann transport equation. The boundary conditions can be taken from spacecraft data, or may be defined from an empirical model; details are found in Chapter 3. With the updated distribution function, the components of the total plasma pressure tensor $\overleftrightarrow{P}$ may be calculated and combined with knowledge of the magnetic field to give the perpendicular drift current $\vec{J}_{\perp}$. Exploiting the zero divergence of total current density, one obtains parallel current density $J_{\|}$and thus the field aligned currents at the upper ionospheric boundary. The ionospheric electric field is then computed through the inductive changes due to the changing magnetic field and the field aligned currents fed into an ionospheric Ohm's Law. Then by using the generalized Ohm's Law, the magnetospheric electric field can be updated through a magnetic field line tracing algorithm. At this point, the algorithm can be iterated forward in time. The mathematics and assumptions associated with each step of this process may be found in the original Vasyliunas $^{4}$ paper.

A host of different inner magnetospheric numerical models exist, all based on the $V a$ syliunas loop to varying degrees, for simulating the physics of Earth's inner magnetosphere. Each model is designed to study specific spatial regions, physical processes, and timescales. 


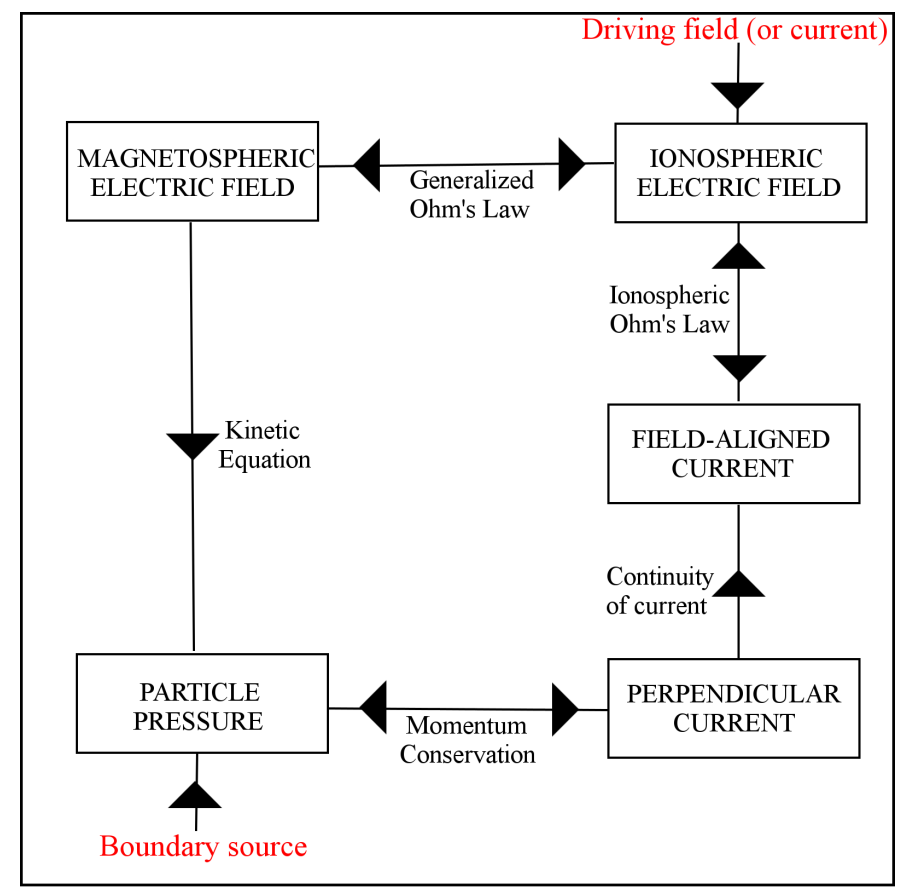

Figure 2.1: Flow chart for the Vasyliunas loop algorithm.

In general there is strong coupling between different magnetospheric regions (suggested by Figure 2.1), but it is not always possible or necessary to simulate every region and interaction within each model. Thus, different numerical models are appropriate for different studies.

One prevailing model, the Rice Convection Model (RCM), ${ }^{7,8}$ is the direct result of one of the earliest attempts to investigate magnetospheric physics using iterative computing. An improved, more complete adaptation of the Vasyliunas loop is shown in Figure 2.2, which is taken from Harel et al. ${ }^{7}$ The authors compute the components of the drift current for discrete energies, and update the magnetic field model to reflect field re-configurations for each substorm phase. In the original work, the electric field was calculated from the ionospheric potential, but RCM was later updated to calculate these self-consistently by including induction. Later, the RCM was generalized to permit anisotropic pitch angle distributions and coupled to the Fok et al. ${ }^{9}$ kinetic model to become a more comprehensive inner magneto- 


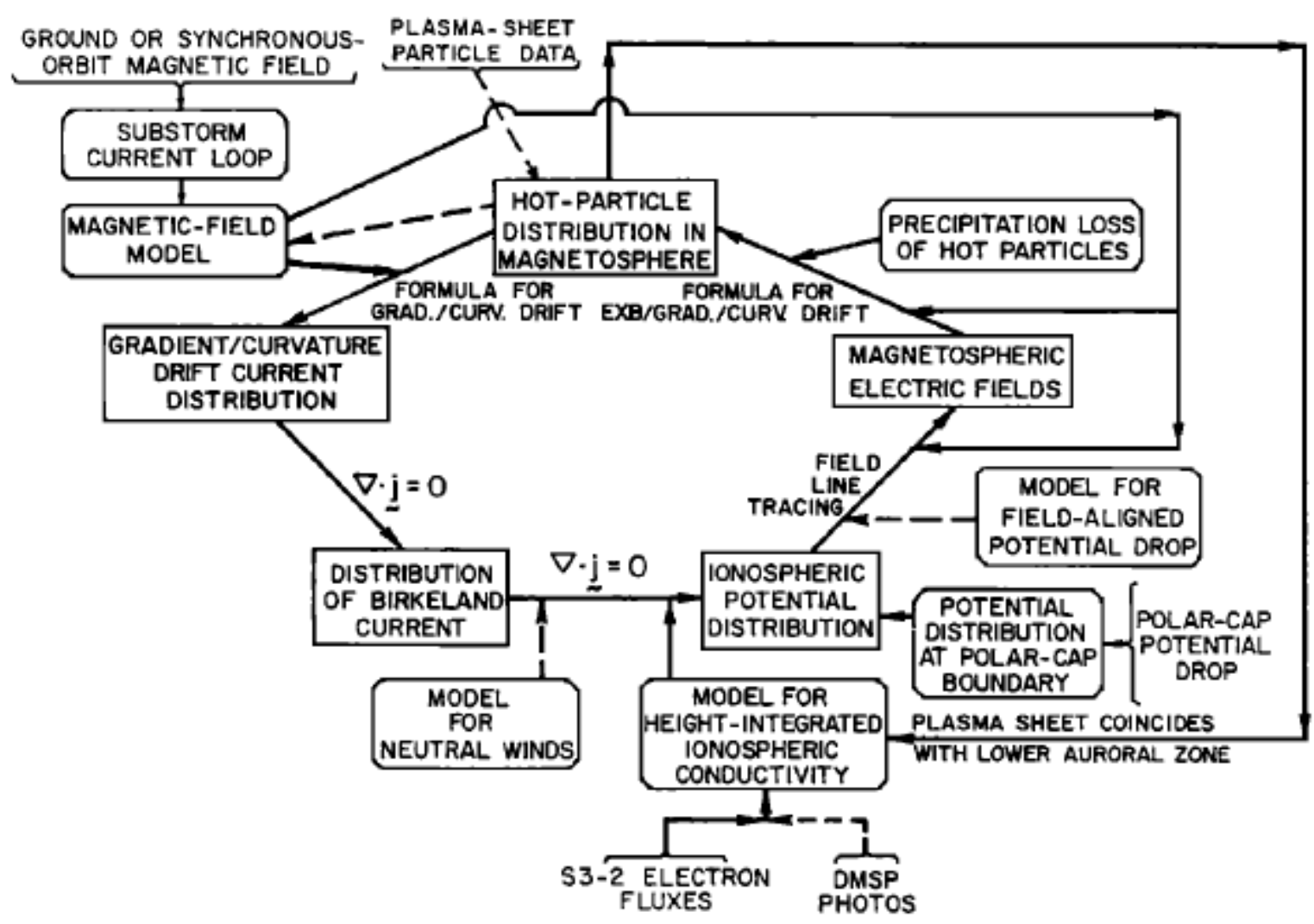

Figure 2.2: Flow chart for the RCM loop algorithm. From Harel et al.

spheric numerical model, called the Comprehensive Ring Current Model (CRCM).

An extensive description of CRCM is given by Fok et al. ${ }^{10}$ The RCM component of the code is responsible for self-consistently calculating electric fields, while the Fok model advances the distribution functions of each species. This is done by solving the bounceaveraged Boltzmann transport equation

$$
\frac{\partial \bar{f}_{\sigma}}{\partial t}+\left\langle\dot{\lambda_{i}}\right\rangle \frac{\partial \bar{f}_{\sigma}}{\partial \lambda_{i}}+\left\langle\dot{\phi}_{i}\right\rangle \frac{\partial \bar{f}_{\sigma}}{\partial \phi_{i}}=-v \sigma_{c x}\left\langle n_{H}\right\rangle \bar{f}_{\sigma}-\left(\frac{\bar{f}_{\sigma}}{0.5 \tau_{b}}\right)_{L C}+\left(\frac{\partial f_{\sigma}}{\partial t}\right)_{\text {boundary }}
$$

for the average phase space density of a given species $\sigma, \bar{f}_{\sigma}$, between mirror points on a field 
line while conserving the first and second adiabatic invariants. Here, $\bar{f}_{\sigma}=\bar{f}_{\sigma}(M, K, \lambda, \phi, t)$, where $M$ and $K$ are the first and second adiabatic invariants, ${ }^{11} \lambda$ is magnetic latitude in the ionosphere, $\phi$ is ionospheric magnetic local time, $t$ is time, and $\langle x\rangle$ indicates a bounceaveraged quantity. The first adiabatic invariant $M$ for a given species $\sigma$ is defined by

$$
M_{\sigma}=\frac{m_{\sigma} v_{\perp}^{2}}{2 B}
$$

where $m_{\sigma}$ is the mass of the particle, $v_{\perp}$ is the perpendicular particle velocity, and $B$ is the local magnetic field strength. The quantity $M$ is considered invariant if changes in the system are slow compared with the gyro-orbit timescales. At the magnetic equator, magnetospheric protons at geosynchronous orbit $\left(6.6 R_{E}\right)$ in a dipole field have a gyroperiod of $T \approx 0.6$ seconds. The second adiabatic invariant has a few interchangeable forms. Here we choose the form commonly referred to as $K$, defined as

$$
K=\oint p_{\|} d s
$$

where the integral is performed along a magnetic field line between the two mirror points. Here $p_{\|}$is the parallel particle momentum. Note that $K=0$ for equatorially mirroring particles, and particles in the loss cone have a $K$ value that diverges to infinity since these particles precipitate into the ionosphere.

The CRCM uses an empirical model to specify the high-latitude electric potential boundary condition, which maps to the magnetic equator to specify the time-dependent cross-tail convection electric field at the outer boundary. In CRCM, the corotation electric field is taken into account but it is not necessary to calculate it self-consistently as there exists a well-known analytical result which may be separated out of the governing Hamiltonian. ${ }^{10}$ 
The CRCM includes charge exchange, loss cone, and magnetopause losses, all shown on the right-hand side (RHS) of equation 2.1; charge exchange decay rates are determined using the Rairden et al. ${ }^{12}$ exospheric neutral hydrogen model, as it has been found to not appreciably deviate from recent, more physical models. ${ }^{13}$ The second term on the RHS accounts for particles within the bounce loss cone, and the final term on the RHS accounts for sources and losses at the simulation boundaries. In those terms, $\sigma_{c x}$ is the energy-dependent charge exchange cross section, $n_{H}$ is the average local neutral hydrogen density and $\tau_{b}$ is the bounce period. The bounce averaging provides a 2-D magnetic-equatorial projection of the particle distribution out to roughly 8-10 $R_{E}$ on the night-side, which corresponds to the dynamic boundary of the model. For the simulations discussed in this manuscript, we use an ionospheric altitude of $120 \mathrm{~km}$, zero dipole tilt angle, the Tsyganenko and Stern 1996 (T96) magnetic field model, ${ }^{14}$ and the Weimer model $^{15}$ for electric potentials at the high latitude boundary, which is just above $69^{\circ}$ magnetic latitude.

The CRCM simulations discussed here have a dynamic outer boundary that is typically located between 8-10 $R_{E}$ during the simulations, corresponding to the closed field line region of the inner magnetosphere. The location of the outer boundary is spatially dynamic because magnetic field lines may change shape in the inner magnetosphere while they remain essentially fixed at ionospheric footpoints. Plasma boundary conditions may be imposed in a number of ways, either by using empirical models or by using event-specific observations; a discussion of temperature boundary conditions typically used in CRCM is found in chapter 3.

Boundary densities are also required to fully specify the time-dependent particle distributions in each boundary cell. For the simulations compared and discussed in Chapter 4, we specify ion density boundary conditions with the Tsyganenko and Mukai model. It 
is necessary to keep density boundary conditions the same for both simulations to isolate the influence that each ion temperature boundary condition model has on the results. The Tsyganenko and Mukai model is a natural choice for boundary densities since one set of simulations uses Tsyganenko and Mukai ion temperatures and the models for $n_{i}$ and $T_{i}$ are calculated from the same set of observational data.

Because CRCM assumes that the first and second adiabatic invariants are conserved, it implicitly assumes that changes in the fields, plasma, or drivers do not occur on gyroperiod or bounce period timescales. Thus, any gyro-resonant or bounce-resonant wave activity cannot be accurately modeled by CRCM. CRCM timescales are primarily those on the order of hours (for Earth's parameters), i.e., plasma drift timescales. A discussion of the three fundamental plasma drifts follows.

Despite the complexity and strong coupling between so many disparate regions and processes, a lot can be learned about the core physics by using analytical approaches. This of course requires simplifying assumptions, but nevertheless useful and reasonable physics emerge. By restricting our time frame of concern to certain intervals 'short' enough, we give ourselves a much more simple mathematical task while still admitting the physics that occurs within such a time interval.

Because of the strong time-dependence and myriad nonlinear effects in the magnetospheresolar wind system, it is very difficult to succeed in the application of analytic methods to the global system. It is true however that one may make simplifying assumptions and learn quite a lot about the behavior of magnetospheric plasma. Here we discuss characteristic properties of plasma particles that remain effectively constant over certain time periods under 'typical' magnetospheric conditions.

By considering the magnetospheric electric and magnetic fields, one may simply calculate 
the drifts of equatorially-mirroring particles and gain some useful insight into the transport processes that govern the distribution of plasma in the inner magnetosphere. Assuming the plasma is perfectly conducting, the electric field is given by

$$
\vec{E}=-\vec{v} \times \vec{B}
$$

Under typical conditions on the nightside in the magnetic equatorial plane, the plasma velocity $\vec{v}$ is Earthward $\left(+\hat{X}_{\mathrm{GSM}}\right)$ and the magnetic field is primarily in the $+\hat{Z}_{\mathrm{GSM}}$, which gives a convection electric field that points from dawn to dusk, which corresponds to the $+\hat{Y}_{\mathrm{GSM}}$ direction. There are three fundamental drifts that particles experience: $\vec{E} \times \vec{B}$ drift, gradient drift, and curvature drift. These drift velocities are given respectively by

$$
\begin{gathered}
\vec{v}_{\vec{E} \times \vec{B}}=\frac{\vec{E} \times \vec{B}}{B^{2}} \\
\vec{v}_{\nabla B}=s_{q} \frac{1}{2} v_{\perp} r_{L} \frac{\vec{B} \times \nabla B}{B^{2}} \\
\vec{v}_{R}=\frac{m v_{\|}^{2}}{q B^{2}} \frac{\vec{R}_{c} \times \vec{B}}{R_{c}^{2}}
\end{gathered}
$$

Here $s_{q}=1$ for positively charged species and $s_{q}=-1$ for negatively charged species, $v_{\perp}$ $\left(v_{\|}\right)$is the magnitude of perpendicular (parallel) component of the particle velocity, $r_{L}$ is the Larmor radius, and $\vec{R}_{c}$ is the radius of curvature vector of the magnetic field $\vec{B}$. Note that $v_{\vec{E} \times \vec{B}}$ has no energy or species dependence, so all magnetospheric particles experience an identical local $\vec{E} \times \vec{B}$ drift. In contrast, the gradient and curvature drifts depend on the particle charge, so electrons and ions drift in different directions. Electrons gradient-curvature 
drift from midnight towards dawn, whereas positively-charged ions gradient-curvature drift from midnight towards dusk. Also note that the gradient-curvature drift depends on particle energy.

\subsection{Computations used for CRCM Data Analysis}

The primary output from CRCM simulations are the particle fluxes $\Gamma_{\sigma}\left(\vec{r}, t, E^{\prime}, \alpha\right)$, which are resolved by species $\sigma$, time $t$, magnetic local time (MLT), latitude $\lambda$ or equatorial radial location $r$, kinetic energy $E^{\prime}$, and pitch angle $\alpha$. Depending on what analysis is being performed, it may be preferable to think of spatial location in terms of latitude and MLT, or in terms of equatorial radius and MLT. For example, it is conventional to look at Birkeland currents (region 2 currents) mapped to the ionosphere, such that latitude is useful, whereas fluxes, pressure and density are typically mapped out to the magnetic equatorial plane so radial location is more appropriate. The two representations are equivalent to the extent that the magnetic field is correct, as the plasma data are mapped along field lines from the ionosphere out to the equatorial grid. Because both representations are useful, both data sets are written out to separate files for easy access. The geocentric solar magnetic (GSM) coordinate system is used in the relevant computations in Chapters 4 and 5. The orientation of this coordinate system is as follows. The $\hat{X}_{\mathrm{GSM}}$ component points sunward from Earth (positive on the dayside, negative on the nightside), the $\hat{Z}_{\mathrm{GSM}}$ component is aligned with

Earth's magnetic dipole axis, and the $\hat{Y}_{\mathrm{GSM}}$ coordinate completes the right-handed system (positive towards dusk).

If instantaneous pitch angle distributions are not important, one may obtain pitch angleaveraged flux $J_{\alpha}$ with the integral 


$$
J_{\alpha}\left(\vec{r}, E^{\prime}, t\right)=\int_{-\pi / 2}^{\pi / 2} \sin (\alpha) d \alpha \Gamma_{\sigma}\left(\vec{r}, E^{\prime}, t, \alpha\right)
$$

The quantity $J_{\alpha}$ in equation 2.8 has dimensions of differential number flux, \# / (area time energy solid-angle). The common convention in space physics is to use $1 /\left(\mathrm{cm}^{2}\right.$ sec $\mathrm{keV}$ sr) for these units. It is often desirable to take moments over plasma distribution functions in order to perform analyses. Since CRCM includes ions and electrons with energies up to hundreds of $\mathrm{keV}$, relativistic effects are important. Beginning with the distribution function $f_{\sigma}(\vec{r}, \vec{p}, t)$, one may compute the particle density with ${ }^{16}$

$$
n(\vec{r}, t)=\int f_{\alpha}(\vec{r}, \vec{p}, t) d^{3} p
$$

where $f_{\alpha}$ is the pitch angle-averaged phase space density. Given a pitch angle-averaged distribution, one may use $d^{3} p=4 \pi p^{2} d p$ and thus obtain

$$
n(\vec{r}, t)=4 \pi \int p^{2} \frac{J_{\alpha}\left(\vec{r}, E^{\prime}, t\right)}{p^{2}} d p
$$

where the relativistically-correct relation between differential particle flux and phase space density

$$
f(\vec{r}, \vec{p}, t)=\frac{J\left(\vec{r}, E^{\prime}, t\right)}{p^{2}}
$$

has been used. ${ }^{16}$ From elementary relativity, ${ }^{17}$ it is well known that $\left(E^{\prime}+E_{0}\right)^{2}=p^{2} c^{2}+E_{0}^{2}$, where $E_{0}=m c^{2}$ is the particle rest energy and $c$ is the speed of light, and thus

$$
p=\frac{1}{c} \sqrt{E^{\prime}} \sqrt{E^{\prime}+2 E_{0}}
$$


and thus the relation between the differential momentum element $d p$ and differential kinetic energy element $d E^{\prime}$ is

$$
d p=\frac{\left(E^{\prime}+E_{0}\right)}{c \sqrt{E^{\prime}} \sqrt{E^{\prime}+2 E_{0}}} d E^{\prime}
$$

which gives

$$
n(\vec{r}, t)=\frac{4 \pi}{c} \int \frac{d E^{\prime}}{\sqrt{E^{\prime}}} \frac{E^{\prime}+E_{0}}{\sqrt{E^{\prime}+2 E_{0}}} J_{\alpha}\left(E^{\prime}, \vec{r}, t\right)
$$

for the density moment. Here the energies are given in $\mathrm{keV}$, the differential number flux in $1 /\left(\mathrm{keV} \mathrm{cm}^{2} \mathrm{sec} \mathrm{sr}\right), c$ in $\mathrm{cm} / \mathrm{s}$, and thus the number density $n$ has units of $1 / \mathrm{cm}^{3}$. Compare this result with the density moment in the non-relativistic limit, given by

$$
n(\vec{r}, t)=2 \pi \sqrt{2 m} \int \frac{d E^{\prime}}{\sqrt{E^{\prime}}} J_{\alpha}\left(E^{\prime}, \vec{r}, t\right)
$$

Other moments, such as temperature or mean kinetic energy may be computed once the density is known. Although the concept of thermodynamic temperature breaks down when the distribution function becomes non-Maxwellian, the concept of 'kinetic temperature' is still useful in describing the spread in velocity, momentum, or energy. ${ }^{18}$ The kinetic temperature $k_{B} T(\vec{r}, t)=P(\vec{r}, t) / n(\vec{r}, t)$ is commonly used, and the well-known relation between mean energy and temperature ${ }^{19}$

$$
k_{B} T(\vec{r}, t)=\frac{2}{3}<E^{\prime}(\vec{r}, t)>=\frac{2}{3 n(\vec{r}, t)} \int d^{3} p E^{\prime} f_{\alpha}(\vec{r}, \vec{p}, t)
$$

is used in both the Maxwellian and non-Maxwellian cases. Substituting the result from equation 2.13, one will obtain 


$$
k_{B} T(\vec{r}, t)=\frac{8 \pi}{3 c n(\vec{r}, t)} \int d E^{\prime} J_{\alpha}(\vec{r}, E, t) \frac{\sqrt{E^{\prime}}\left(E^{\prime}+E_{0}\right)}{\sqrt{E^{\prime}+2 E_{0}}}
$$

Compare this with the non-relativistic temperature moment, given by

$$
k_{B} T(\vec{r}, t)=\frac{4 \pi \sqrt{2 m}}{3 n(\vec{r}, t)} \int d E^{\prime} \sqrt{E^{\prime}} J_{\alpha}\left(\vec{r}, E^{\prime}, t\right)
$$

In both equation 2.17 and 2.18 , the temperature $k_{B} T$ has units of keV, provided cgs units are used for $c$ and $n$ and energies are also expressed in $\mathrm{keV}$.

The relativistic pressure $P(\vec{r}, t)$ is readily calculated with $P(\vec{r}, t)=n(\vec{r}, t) k_{B} T(\vec{r}, t)$, resulting in

$$
P(\vec{r}, t)=\frac{8 \pi}{3 c} \int d E^{\prime} \sqrt{E^{\prime}} J_{\alpha}\left(E^{\prime}, \vec{r}, t\right) \frac{E^{\prime}+E_{0}}{\sqrt{E^{\prime}+2 E_{0}}}
$$

for the pressure moment with units of $\mathrm{keV} / \mathrm{cm}^{3}$. To convert to units of nanoPascals $\left(10^{-9}\right.$ Joules $/ \mathrm{m}^{3}$ ), which is the conventional choice, a prefactor is multiplied through giving

$$
P(\vec{r}, t)=\frac{8 \pi e 10^{15}}{3 c} \int d E^{\prime} \sqrt{E^{\prime}} J_{\alpha}\left(E^{\prime}, \vec{r}, t\right) \frac{E^{\prime}+E_{0}}{\sqrt{E^{\prime}+2 E_{0}}}
$$

where $e$ is the proton charge.

The moment calculations shown in equations 2.14, 2.17 and 2.20 are used in Chapter 4 for processing data generated from CRCM for geomagnetic storm simulations. Those integrations are normally truncated to energy bounds between $E_{1}$ and $E_{2}$, usually corresponding to the low-energy portion of the energy spectrum with $E_{1} \approx 0 \mathrm{keV}$ and $E_{2} \approx 25-40 \mathrm{keV}$. Details on specific integration bounds for different spacecraft measurements and simulation results are discussed in chapters 3 and 4 . 


\subsection{Spacecraft Observations}

\subsubsection{TWINS}

The Two Wide-Angle Imaging Neutral-Atom Spectrometers (TWINS), ${ }^{20}$ a NASA Explorer mission of opportunity, consists of a pair of satellites (TWINS 1 and 2) in high-altitude, high-inclination Molniya orbits. Each satellite images Earth's magnetosphere independently, although stereoscopic, 3-D imaging has been a key feature of the TWINS mission. Each spacecraft contains a Lyman- $\alpha$ detector for measurements of geocoronal neutral hydrogen, environmental sensors that measure the local charged particle environment, and an energetic neutral atom (ENA) imager for remote sensing of neutral atoms through charge exchange with ions. The ENA imager on each spacecraft houses two independent heads, referred to as 'towards' and 'away' heads. The assembly is mounted on a rotating actuator which sweeps out $180^{\circ}$ every 60 seconds in a back-and-forth motion, sweeping out an approximately Earthcentered viewing cone. Figure 2.3 shows the TWINS instrument on the left, and illustrates the field of view (FOV) when deployed on the host spacecraft (right). The time-of-flight resolved $1-100 \mathrm{keV}$ ENA spectrum is detected by the TWINS ENA imagers with $4^{\circ}$ by $4^{\circ}$ angular resolution and 1-2 minute time resolution. An illustration of the TWINS orbits is shown in Figure 2.4; the three-axis stabilized spacecraft have an orbital period of just under 12 hours. The perigee and apogee altitudes are $\sim 1000 \mathrm{~km}$ and $\sim 7.2 \mathrm{R}_{E}$, respectively.

Early observations of anomalies in in situ ion particle data prompted the idea of using energetic neutral atoms to secondarily image magnetospheric ion populations through the charge exchange process. ${ }^{21,22}$ The Astrid microsatellite mission ${ }^{23}$ provided the first publicly accessible proof of concept for this method of imaging the terrestrial magnetosphere. ${ }^{24}$ This mission was the first to include a suite of two dedicated, onboard ENA imagers; the satellite 


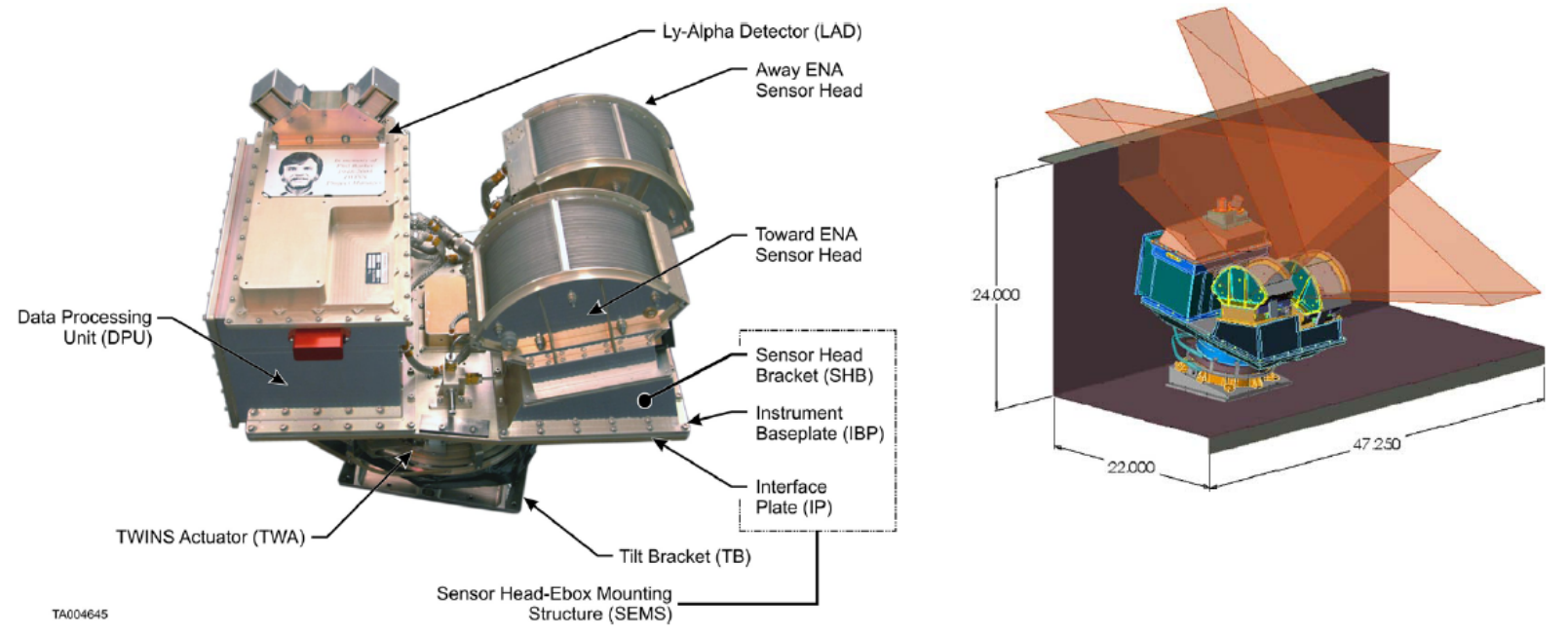

Figure 2.3: The TWINS instrument (left), showing the actuator and two heads. A depiction of the TWINS instrument when deployed, illustrating the FOV during operation. From McComas et al. ${ }^{20}$

flew in a circular polar orbit at $1000 \mathrm{~km}$ altitude and remained operational for 5 weeks. Astrid's ENA imagers covered the 13-140 keV and 0.1-70 keV energy ranges and operated with angular resolutions of $2.5^{\circ}$ by $25^{\circ}$ and $4.6^{\circ}$ by $11.5^{\circ}$, respectively. Later, the reality that more advanced space-based ENA imagers with better energy and angular resolution could realistically be deployed in Earth's magnetosphere came to fruition with the Imager for Magnetopause-to-Aurora Global Exploration (IMAGE) mission, ${ }^{25}$ which launched in 2000 and continued to transmit data until the end of 2005. Aboard the IMAGE spacecraft was a suite of ENA imagers, which constituted the first successful long-term attempt at continuous ENA imaging within Earth's magnetosphere. The Low-Energy, Medium-Energy, and HighEnergy Neutral Atom Imagers (LENA, ${ }^{26}$ MENA, ${ }^{27}$ and HENA ${ }^{28}$ respectively) covered the 10-500 eV, 1-30 keV, and 10-500 keV energy ranges and operated with typical angular resolution of $4^{\circ}$ by $4^{\circ}$ (the same as TWINS). The TWINS ENA imagers are closely based 


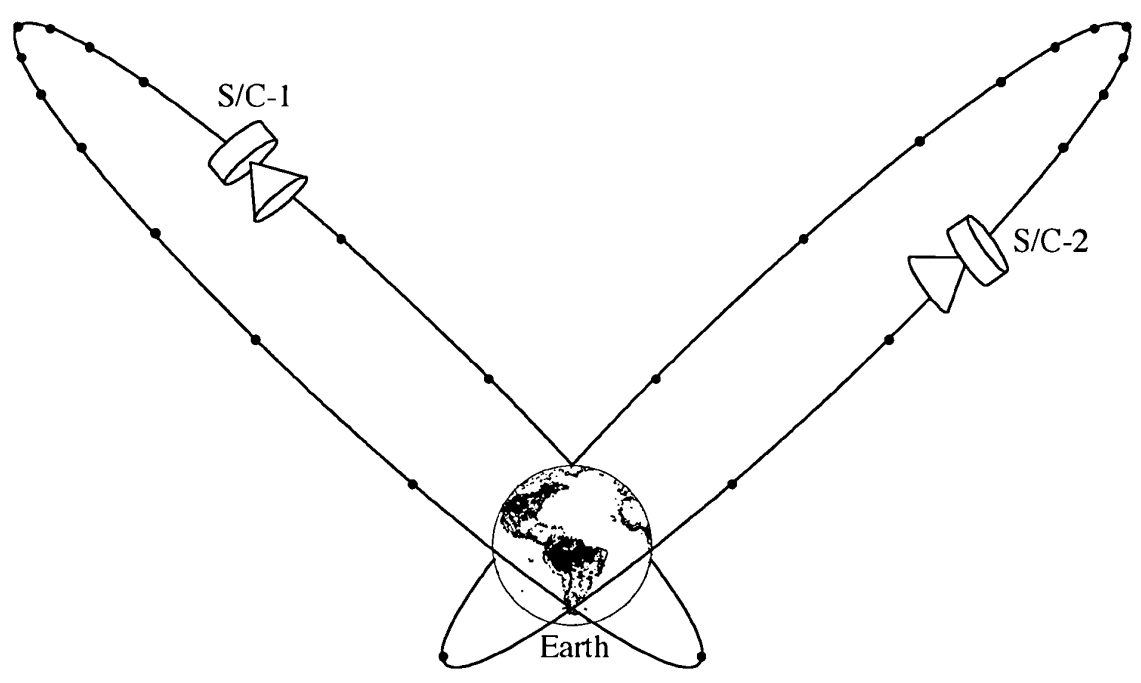

Figure 2.4: Sketch of the Molniya orbits of both TWINS spacecraft, from McComas et $a{ }^{20}$

on IMAGE/MENA instrument design, although improvements were made to the TWINS sensor heads to offer higher sensitivity and improved mechanical design. ${ }^{20}$

Given the TWINS instruments' wide fields of view and angular resolution, maps of energyresolved ENA measurements can be used to infer magnetospheric ion temperatures through fits of ENA intensity versus energy. Scime et $a l .{ }^{29}$ first presented temperature maps based on MENA instrument data, which were found to be in agreement with in situ observations at geosynchronous orbit and also with temperatures computed through full inversions of the same MENA data set. ${ }^{30}$ Zaniewski et $a l .{ }^{31}$ applied this technique in a superposed epoch analysis of 39 geomagnetic storms and derived statistically-averaged maps binned by substorm phases; this study provided the space physics community with the first glimpse into global magnetospheric response using ENA imaging. The time-resolved ion temperatures can be calculated at nearly any location and time, provided that ENA counting statistics meet significance requirements. This is accomplished by integrating measured fluxes for each instrument pixel over multiple instrument actuator sweeps. The time integration interval is 
adjusted to obtain good counting statistics, with a trade-off between fast imaging and lower uncertainty; typical integration intervals are 20-60 minutes. Our method, as applied to the TWINS data, sets a fixed bin size at $0.5 R_{E}$ by $0.5 R_{E}$ in the magnetic equatorial plane (GSM coordinates) that is filled by mapping the ENA flux along the line of sight of each instrument pixel. The details of this procedure are outlined further in Chapter 3.3.

See Brandt et al. ${ }^{32}$ for an overview of the relatively short history of ENA imaging, and thus for the background context upon which the TWINS mission is built. See Goldstein and McComas ${ }^{33}$ for an overview of research conducted in connection with TWINS data products during the first five years of operation, 2008-2013.

\subsubsection{THEMIS}

The Time History of Events and Macroscale Interactions during Substorms (THEMIS) mission $^{34}$ consists of 5 spacecraft, each of which is outfitted with an identical instrument suite. In addition to the spacecraft constellation, ground-based arrays and All-Sky Imagers located in North America are used extensively in mapping and correlating space-based observations with phenomena at Earth. ${ }^{35,36}$ The primary objectives of the THEMIS mission are to establish when and where substorms begin, determine how the individual components of the substorm interact, determine how substorms power the aurora, and identify how the localized current disruptions couple to global substorm phenomena.

A principle aim of the THEMIS mission is to provide scientific data products that distinguish between the two prevailing substorm models; the current-disruption model proposes that a cross-tail current disruption in the near-Earth magnetotail begins the substorm process ${ }^{37}$ while the near-Earth neutral line model proposes that magnetotail reconnection initiates the process and subsequently leads to the current disruption and substorm onset. ${ }^{38}$ 
This is a long-standing and controversial topic within the space physics community and has proven to be a very difficult problem to fully address. Outside of specifically investigating substorms, the THEMIS mission has proven to be extremely useful in providing in situ data for general purposes; this is especially true given the fact that LANL MPA and SOPA geosynchronous data has not been available since 2007.

Each of the five THEMIS spacecraft are outfitted with ion and electron electrostatic energy analyzers (ESA), ${ }^{39}$ ion and electron solid state telescopes (SST), ${ }^{34}$ electric field instruments (EFI), ${ }^{40}$ search coil magnetometers $(\mathrm{SCM}){ }^{41}$ and fluxgate magnetometers (FGM). ${ }^{42}$

Separate ESA instrument heads measure ions and electrons in the energy range of $\sim 1$ $\mathrm{eV}-25 \mathrm{keV}$ (ions) and $\sim 1 \mathrm{eV}-30 \mathrm{keV}$ (electrons). The SST contains two heads, one for electrons and one for ions, and measures superthermal particles in the $25 \mathrm{keV}-6 \mathrm{MeV}$ energy range. The SST, like the ESA instrument, resolves by energy and solid angle. These two instruments have 3 operational modes for taking plasma measurements: full mode, burst mode, and reduced mode. In full mode, particle distributions are measured with high angular resolution and with time resolution of a few minutes. In burst mode, particle measurements are taken with high angular resolution and time resolution of $\sim 1$ second, but this mode is only activated in short, intermittent intervals. In reduced mode, particle measurements are taken with low angular resolution and $\sim 3$ second time resolution. In this research, the ESA and SST are of particular interest, as they allow us to measure energy spectra of plasma constituents. These in situ measurements may then be compared with simulation results of real events. Specifically, time-resolved in situ energy spectra obtained by combining ESA and SST measurements can be compared with energy spectra predicted by numerical models. Also, plasma moments may be taken over observed energy spectra, providing in situ densities, pressures and temperatures which may be compared with moments taken from simulation 
data. Such comparisons are shown in Chapter 4.

The THEMIS Data Analysis Suite (TDAS) is a comprehensive, IDL-based access and analysis package for THEMIS data; details regarding use, installation, and numerical routines contained therein may be found through 'themis.ssl.berkeley.edu'. TDAS gives access to all THEMIS data products, including all three data modes and moments taken for each mode. TDAS also permits convenient manipulation of THEMIS data. For example, TDAS allows users to easily remove sunlight contamination from SST energy spectra, and remove background ion populations from ESA data. THEMIS data obtained through TDAS will be used for data-model comparisons in conjunction with simulated CRCM data in Chapter 4.

Readers are referred to the Angelopoulos $^{34}$ review article (and references therein) for a complete discussion of the importance and context of the THEMIS mission, the fine details of the on-board instrumentation, and the mission's role in resolving open questions in the ongoing substorm debate. 


\section{References}

[1] W. I. Axford and C. O. Hines, "A unifying theory of high-latitude geophysical phenomena and geomagnetic storms," Canadian Journal of Physics, vol. 39, no. 10, pp. 1433$1464,1961$.

[2] J. W. Dungey, "Interplanetary magnetic field and the auroral zones," Physical Review Letters, vol. 6, p. 47, 1961.

[3] J. A. Fejer, "Theory of the geomagnetic daily disturbance variations," Journal of Geophysical Research, vol. 69, no. 1, pp. 123-137, 1964.

[4] V. M. Vasyliunas, "Mathematical models of magnetospheric convection and its coupling to the ionosphere," in Particles and Fields in the Magnetosphere (B. McCormac, ed.), pp. 60-71, D. Reidel, Norwell, Mass., 1970.

[5] V. M. Vasyliunas, The Earth's Magnetospheric Processes, ch. The interrelationship of magnetospheric processes, pp. 29-38. D. Reidel Pub. Comp., Dordrecht, 1972.

[6] R. A. Wolf, "Effects of ionospheric conductivity on convective flow of plasma in the magnetosphere," Journal of Geophysical Research, vol. 75, no. 25, pp. 4677-4698, 1970.

[7] M. Harel, R. A. Wolf, P. H. Reiff, and M. Smiddy, Quantitative Modeling of Magnetospheric Processes, vol. 21 of Geophysical Monograph, ch. Computer Modeling of Events in the Inner Magnetosphere, pp. 499-512. American Geophysical Union, 1979.

[8] M. Harel, R. A. Wolf, P. H. Reiff, R. W. Spiro, W. J. Burke, F. J. Rich, and M. Smiddy, "Quantitative simulation of a magnetospheric substorm," Journal of Geophysical Research, vol. 86, pp. 2217-2241, 1981. 
[9] M. C. Fok and T. E. Moore, "Ring current modeling in a realistic magnetic field configuration," Geophysical Research Letters, vol. 24, pp. 1775-1778, 1997.

[10] M. C. Fok, R. A. Wolf, R. W. Spiro, and T. E. Moore, "Comprehensive computational model of earth's ring current," Journal of Geophysical Research, vol. 106, no. A5, p. $8417,2001$.

[11] J. G. Roederer, Dynamics of geomagnetically trapped radiation. Springer-Verlag, 1970.

[12] R. L. Rairden, L. A. Frank, and J. D. Craven, "Geocoronal imaging with dynamics explorer," Journal of Geophysical Research, vol. 91, no. A12, pp. 13613-13630, 1986.

[13] R. Ilie, R. M. Skoug, H. O. Funsten, M. W. Liemohn, J. J. Bailey, and M. Gruntman, "The impact of geocoronal density on ring current development," Journal of Atmospheric and Solar-Terrestrial Physics, vol. 99, pp. 92-103, 2012.

[14] N. A. Tsyganenko and D. P. Stern, "Modeling the global magnetic field of the largescale birkeland current systems," Journal of Geophysical Research, vol. 101, no. A12, pp. 27187-27198, 1996.

[15] D. R. Weimer, "An improved model of ionospheric electric potentials including substorm perturbations and applications to the geospace environment modeling november 24, 1996, event," Journal of Geophysical Research, vol. 106, no. A1, pp. 407-416, 2001.

[16] M.-B. Kallenrode, Space Physics: An Introduction to Plasmas and Particles in the Heliosphere and Magnetospheres. Springer, 3rd ed., 2004.

[17] W. G. V. Rosser, Introductory Relativity. Butterworth and Co., 1967. 
[18] W. Baumjohann and R. A. Treumann, Basic Space Plasma Physics. Imperial College Press, 1997.

[19] R. K. Pathria, Statistical Mechanics. Elsevier, 2nd ed., 2006.

[20] D. J. McComas, F. Allegrini, J. Baldonado, B. Blake, P. C. Brandt, J. Burch, J. Clemmons, W. Crain, D. Delapp, R. DeMajistre, D. Everett, H. Fahr, L. Friesen, H. Funsten, J. Goldstein, M. Gruntman, R. Harbaugh, R. Harper, H. Henkel, C. Holmlund, G. Lay, D. Mabry, D. Mitchell, U. Nass, C. Pollock, S. Pope, M. Reno, S. Ritzau, E. Roelof, E. Scime, M. Sivjee, R. Skoug, T. S. Sotirelis, M. Thomsen, C. Urdiales, P. Valek, K. Viherkanto, S. Weidner, T. Ylikorpi, M. Young, and J. Zoennchen, "The two wideangle imaging neutral-atom spectrometers (twins) nasa mission-of-opportunity," Space Science Reviews, vol. 142, pp. 157-231, 2009.

[21] E. C. Roelof, D. G. Mitchell, and D. J. Williams, "Energetic neutral atoms (e near $50 \mathrm{kev)} \mathrm{from} \mathrm{the} \mathrm{ring} \mathrm{current:} \mathrm{Imp} \mathrm{7/8} \mathrm{and} \mathrm{isee} \mathrm{1,"} \mathrm{Journal} \mathrm{of} \mathrm{Geophysical} \mathrm{Research,}$ vol. 90, no. A11, pp. 10991-11008, 1985.

[22] D. Hovestadt and M. Scholer, "Radiation belt-produced energetic hydrogen in interplanetary space," Journal of Geophysical Research, vol. 81, p. 5039, 1976.

[23] S. Grahn and A. Rathsman, "Astrid: An attempt to make the microsatellite a useful tool for space science," in Proc. of the 11th Annual AIAA/USU Conf. on Small Satellites, (Logan, Utah), September 1995.

[24] S. Barabash, O. Norberg, R. Lundin, S. Olsen, K. Lundin, P. C. Brandt, E. C. Roelof, C. J. Chase, B. H. Mauk, H. Koskinen, and J. Ryno, Measurement Techniques in Space Plasmas: Fields, ch. Energetic Neutral Atom Imager on the Swedish Micro Satellite 
Astrid, pp. 257-262. No. 103 in Geophysical Monograph Series, American Geophysical Union, 1998.

[25] J. L. Burch, "Image mission overview," Space Science Reviews, vol. 91, pp. 1-14, 2000.

[26] T. E. Moore, D. J. Chornay, M. R. Collier, F. A. Herrero, J. Johnson, M. A. Johnson, J. W. Keller, J. F. Laudadio, J. F. Lobell, K. W. Ogilvie, and P. Rozmarynowski, "The low energy neutral atom imager for image," Space Science Reviews, vol. 91, pp. 155-195, 2000.

[27] C. J. Pollock, K. Asamura, J. Baldonade, M. M. Balkey, P. Parker, J. L. Burch, E. J. Korpela, J. Cravens, G. Dirks, M. C. Fok, H. O. Funsten, M. Grande, M. Gruntman, J. Hanley, J. M. Jahn, M. Jenkins, M. Lampton, M. Marckwordt, D. J. McComas, T. Mukai, G. Penegor, S. Pope, S. Ritzau, M. L. Schattenburg, E. Scime, R. Skoug, W. Spurgeon, T. Stecklein, S. Storms, C. Urdiales, P. Valek, J. T. M. van Beek, S. E. Weidner, M. Wuest, M. K. Young, and C. Zinsmeyer, "Medium energy neutral atom (mena) iimage for the image mission," Space Science Reviews, IMAGE Special Issue, vol. 91, pp. 113-154, 2000.

[28] D. G. Mitchell, S. E. Jaskulek, C. E. Schlemm, E. P. Keath, R. E. Thompson, B. E. Tossman, J. D. Boldt, J. R. Hayes, G. B. Andrews, and N. Paschalidis, "High energy neutral atom (hena) imager for the image mission," Space Science Reviews, vol. 91, pp. $67-112,2000$.

[29] E. E. Scime, A. M. Keesee, J. M. Jahn, J. L. Kline, C. J. Pollock, and M. Thomsen, "Remote ion temperature measurements of earth's magnetosphere: Medium energy 
neutral atom (mena) images," Geophysical Research Letters, vol. 29, no. 10, p. 1438, 2002.

[30] X. X. Zhang, J. D. Perez, T. Chen, C. Wang, P. C. Brandt, D. G. Mitchell, and Y. L. Yang, "Proton temperatures in the ring current from ena images and in situ measurements," Geophysical Research Letters, vol. 32, no. L16101, 2005.

[31] A. M. Zaniewski, X. Sun, A. Gripper, E. E. Scime, J. M. Jahn, and C. J. Pollock, "Evolution of remotely measured inner magnetospheric ion temperatures during a geomagnetic storm," Journal of Geophysical Research, vol. 111, no. A10221, pp. 1-11, 2006.

[32] P. C. Brandt, D. G. Mitchell, E. C. Roelof, S. M. Krimigis, C. P. Paranicas, B. H. Mauk, J. Saur, and R. DeMajistre, "Ena imaging: See the invisible," Johns Hopkins APL Technical Digest, vol. 26, no. 2, p. 143, 2005.

[33] J. Goldstein and D. J. McComas, "Five years of stereo magnetospheric imaging by twins," Space Science Reviews, vol. 180, pp. 39-70, 2013.

[34] V. Angelopoulos, "The themis mission," Space Science Reviews, vol. 141, pp. 5-34, 2008.

[35] C. T. Russell, P. J. Chi, D. J. Dearborn, Y. S. Ge, B. Kuo-Tiong, J. D. Means, D. R. Pierce, K. M. Rowe, and R. C. Snare, "Themis ground-based magnetometers," Space Science Reviews, vol. 141, pp. 389-412, 2008.

[36] S. B. Mende, S. E. Harris, H. U. Frey, V. Angelopoulos, C. T. Russell, E. Donovan, B. Jackel, M. Greffen, and L. M. Peticolas, "The themis array of ground-based observa- 
tories for the study of auroral substorms," Space Science Reviews, vol. 141, pp. 357-387, 2008.

[37] A. T. Y. Lui, R. E. Lopez, S. M. Krimigis, R. W. McEntire, L. J. Zanetti, and T. A. Potemra, "A case study of magnetotail current sheet disruption and diversion," Geophysical Research Letters, vol. 15, no. 7, pp. 721-724, 1988.

[38] D. N. Baker, T. I. Pulkkinen, V. Angelopoulos, W. Baumjohann, and R. L. McPherron, "Neutral line model of substorms: Past results and present view," Journal of Geophysical Research: Space Physics, vol. 101, no. A6, pp. 12975-13010, 1996.

[39] J. P. McFadden, C. W. Carlson, D. Larson, M. Ludlam, R. Abiad, B. Elliott, P. Turin, M. Marckwordt, and V. Angelopoulos, "The themis esa plasma instrument and in-flight calibration," Space Science Reviews, vol. 141, pp. 277-302, 2008.

[40] J. W. Bonnell, F. S. Mozer, G. T. Delory, A. J. Hull, R. E. Ergun, C. M. Culley, V. Angelopoulos, and P. R. Harvey, "The electric field instrument (efi) for themis," Space Science Reviews, vol. 141, pp. 303-0341, 2008.

[41] A. Roux, O. L. Contel, C. Coillot, A. Bouabdellah, B. de la Porte, D. Alison, S. Ruocco, and M. C. Vassal, "The search coil magnetometer for themis," Space Science Reviews, vol. 141, pp. 265-275, 2008.

[42] H. U. Auster, K. H. Glassmeier, W. Magnes, O. Aydogar, D. Constantinescu, D. Fischer, K. H. Fornacon, E. Georgescu, P. Harvey, O. Hillenmaier, R. Kroth, M. Ludlam, Y. Narita, K. Okrafka, F. Plaschke, I. Richter, H. Schwarzi, B. Stoll, A. Valavanoglu, and M. Wiedemann, "The themis fluxgate magnetometer," Space Science Reviews, vol. 141, pp. 235-264, 2008. 


\section{Chapter 3}

\section{Implementation of Boundary}

\section{Conditions}

\subsection{Background}

Boundary conditions are critically important when using numerical models to probe physical systems. In plasma simulations, users must typically define field and particle boundary conditions appropriate for a given system. For example, there may be plasma sources or losses at a boundary, or changes in the magnetic and electric field configurations. In this regard, boundary conditions may be applied to represent physical processes that occur outside of a simulation domain but still have significant impacts within the simulation domain. Non-adiabatic processes such as substorm activity and magnetic reconnection in the magnetotail can trigger localized heating and bulk flows, ${ }^{1,2}$ which are convected through the inner magnetosphere and contribute to the ring current. To accurately represent such processes, appropriate plasma and field boundary conditions must be supplied. ${ }^{3}$ For inner magneto- 
spheric simulations, such as CRCM, such processes are ultimately driven by solar wind forcing and resultant plasma sheet and magnetotail dynamics. Yang et $a{ }^{4}{ }^{4}$ and Liemohn et al. ${ }^{5}$ performed simulations of the 18 April 2002 global sawtooth oscillation event while not actually simulating the downtail event per se. Yang et al. carefully calculated periodic signatures in plasma sheet density and temperature and superimposed these signatures onto outer boundary conditions computed from a mixture of empirical models discussed in sections 3.2.1-3.2.2. Liemohn et al. performed simulations of the same event while supplying boundary conditions provided by time-resolved in situ observations from geosynchronous orbit.

In addition to using boundary conditions to effectively simulate the effects of impulsive external drivers, like tail reconnection events, boundary conditions also play an important role in understanding how weaker, transient plasma sheet features affect the ring current, and also how the ring current responds to time-dependent features during geomagnetic storms. Thus, supplying different boundary conditions to an inner magnetospheric model can result in fundamentally different outputs, and can provide insights into the re-distribution of plasma sheet populations within the inner magnetosphere. In section 3.2, attention is called to existing boundary condition models commonly used in magnetospheric simulations. Outlined in section 3.3 is the theory behind a novel method which is central to this dissertation; this method uses charge exchange imaging to construct global, 2-D ion temperature maps and introduces vast improvements over existing empirically-derived boundary condition models. 


\subsection{Empirical Boundary Conditions in Space Plasma} Simulations

Plasmas are, perhaps surprisingly, often found to obey relatively simple statistical distributions, such as Maxwell-Boltzmann and kappa distributions. This is the case in many plasmas found in Earth's magnetosphere and in the solar wind, including the case of low collisionality. ${ }^{6}$ A phase space description of an isotropic, 3D Maxwell-Boltzmann distribution function for a species $\sigma$ is given by

$$
f_{\sigma}(\vec{r}, \vec{v}, t)=\frac{n_{\sigma}(\vec{r}, t)}{\left(2 \pi k_{B} T_{\sigma}(\vec{r}, t) / m_{\sigma}\right)^{3 / 2}} \exp \left[\frac{-m_{\sigma}\left(\vec{v}-\vec{u}_{\sigma}(\vec{r}, t)\right)^{2}}{k_{B} T_{\sigma}(\vec{r}, t)}\right]
$$

where $n_{\sigma}$ is the local number density of species $\sigma, k_{B}$ is Boltzmann's constant, $T_{\sigma}$ is the local temperature, $\vec{r}$ is the spatial independent variable, $\vec{v}$ is the velocity independent variable, $\vec{u}(\vec{r}, t)$ is the drift velocity vector, $t$ is the temporal independent variable, $m_{\sigma}$ is the particle

mass for species $\sigma$, and $f_{\sigma}$ is the phase space density. A further generalization follows, which is of frequent interest in space plasmas. For a drifting, gyrotropic distribution with different parallel and perpendicular temperatures (relative to the local direction of the magnetic field), $T_{\|}$and $T_{\perp}$ respectively, the distribution function is given by ${ }^{7}$

$$
f_{\sigma}(\vec{r}, \vec{v}, t)=\frac{\left(m_{\sigma} / 2 \pi k_{B}\right)^{3 / 2} n_{\sigma}(\vec{r}, t)}{T_{\sigma, \perp}(\vec{r}, t) \sqrt{T_{\sigma, \|}(\vec{r}, t)}} \exp \left[\frac{-m_{\sigma}\left(v_{\|}-u_{\sigma, \|}\right)^{2}}{2 k_{B} T_{\sigma, \|}(\vec{r}, t)}\right] \exp \left[\frac{-m_{\sigma}\left(\vec{v}_{\perp}-\vec{u}_{\sigma, \perp}\right)^{2}}{k_{B} T_{\sigma, \perp}(\vec{r}, t)}\right]
$$

It is important to note that when parallel and perpendicular temperatures are equal, one recovers the isotropic distribution given in Equation 3.1.

A kappa distribution is described by ${ }^{8}$ 


$$
f_{\kappa, \sigma}(\vec{r}, \vec{v}, t)=\frac{n_{\sigma}(\vec{r}, t)}{2 \pi\left(\kappa w_{\kappa, \sigma}^{2}\right)^{3 / 2}} \frac{\Gamma(\kappa+1)}{\Gamma\left(\frac{\kappa-1}{2}\right) \Gamma(3 / 2)}\left(1+\frac{v^{2}}{\kappa w_{\kappa, \sigma}^{2}}\right)^{-(\kappa+1)}
$$

where the spectral index $\kappa$ controls the slope of the suprathermal tail, $\Gamma(x)$ is the gamma function, and $w_{\kappa, \sigma}=\sqrt{(2 \kappa-3) k_{B} T_{\sigma}(\vec{r}, t) / \kappa m_{\sigma}}$. Kappas are quite similar to a MaxwellBoltzmann distribution; the characteristic difference is the suprathermal tail present in kappa distributions. In Figure 3.1, a comparison is shown between a Maxwellian (red) and 4 different kappa distributions (black, blue, green, yellow); Figure 3.1 is from Pierrard and Lazar. ${ }^{8}$ All distributions are normalized to $\left.f(v)\right|_{v=0}=1$, and the velocity (x-axis) is given in terms of the thermal speed $\sqrt{k_{B} T / m}$. As usual the spread of the distribution in velocity or energy is a proxy for the temperature. From the definition of $\omega_{\kappa, \sigma}$ following equation 3.3, it may readily be inferred that $f(v)$ is not well-defined for $\kappa \leq \frac{3}{2}$. Also, as $\kappa$ approaches infinity a kappa distribution $f_{\infty, \sigma}$ becomes a Maxwellian distribution.

Space-based instruments cannot directly measure particle distribution functions. Instead, particle fluxes $J(E, \Omega, \vec{r}, t, \alpha)$ are typically measured which are resolved in position $\vec{r}$, time $t$, energy $E$, pitch angle $\alpha$ and solid angle $\Omega$. The pitch-angle averaged differential particle flux is related exactly to the particle distribution function through equation 2.11, and therefore the two descriptions may be considered interchangeable.

The plasma sheet is the primary plasma source for the inner magnetosphere models, and thus it is important to capture realistic spatiotemporal variations in that region to understand the connection between the character of the plasma sheet and the resulting makeup of the storm-time ring current. Historically, there have been many attempts to understand how the character of the plasma sheet correlates to solar activity, including Baumjohann et al., ${ }^{1}$ Angelopoulos et al., ${ }^{2}$ Goertz and Baumjohann,${ }^{9}$ and Lennartsson and Shelley. ${ }^{10}$ Additional, 


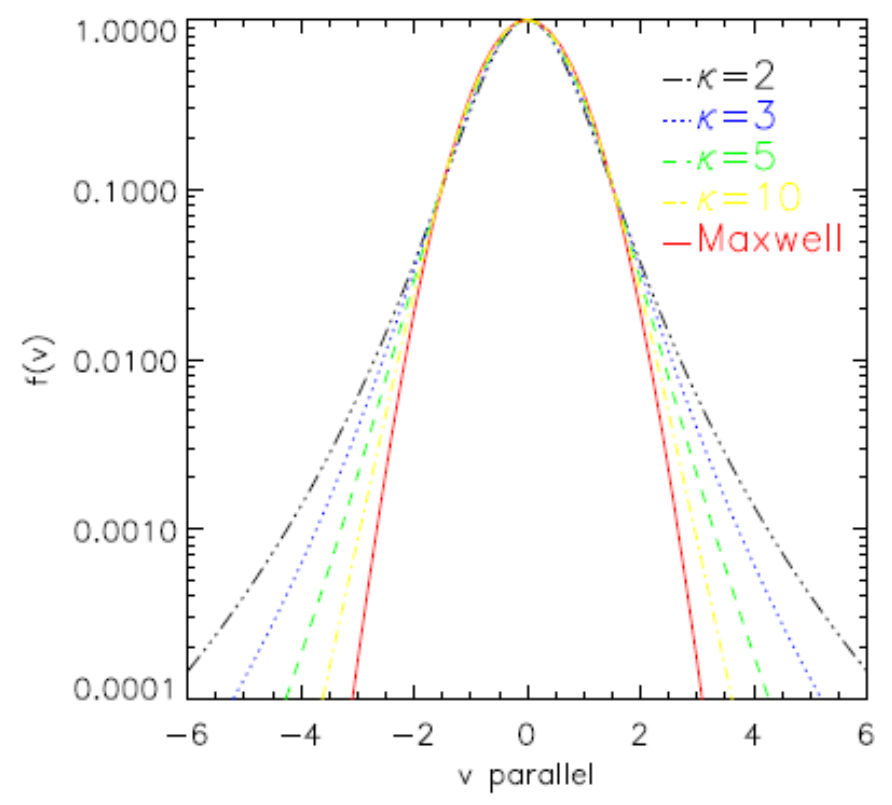

Figure 3.1: Comparison of a Maxwellian distribution function with various kappa distributions. Figure taken from Pierrard and Lazar. ${ }^{8}$

more recent models have been developed using a variety of observations and are discussed in the following sections.

\subsubsection{Borovsky Model}

The Borovsky et al. statistical model ${ }^{11}$ was developed to understand the connection between the solar wind plasma and different plasma sheet regions by comparing simultaneous observations of two different groups of satellites. First, Fast Plasma Experiment ${ }^{12}$ and fluxgate magnetometer ${ }^{13}$ measurements from ISEE-2 in the central plasma sheet were correlated with Solar Wind Plasma Experiment ${ }^{14}$ and helium magnetometer ${ }^{15}$ observations from ISEE-3 in the upstream solar wind. The neutral sheet encounters were restricted to downtail distances between 17.5 and $22.5 R_{E}$ and the neutral sheet was chosen so that encounters with the nearby plasma sheet boundary layer and low latitude boundary layer were filtered out of 
the data set. Second, geosynchronous Magnetospheric Plasma Analyzer ${ }^{16}$ and Synchronous Orbit Particle Analyzer measurements from the 1989-046, 1990-095, 1991-080, and 1994-084 LANL satellites made in the midnight, near-Earth plasma sheet were correlated with solar wind observations from the solar Plasma Faraday Cup aboard IMP-8 and the Solar Wind Experiment $^{17}$ on the WIND mission. The ISEE-2 and -3 data set included March and April 1979, and the LANL/IMP-8/WIND data set covered November 1993 through January 1994.

Of particular concern to inner magnetospheric modeling efforts, specifically for supplying outer boundary conditions to inner magnetospheric models, are the plasma sheet density $n_{p s}$ and plasma sheet temperature $T_{p s}$. The Borovsky et al. study found that for these intervals, the magnetotail neutral sheet temperature obeyed

$$
T_{p s}=-3.65+0.0190 v_{s w}
$$

where $T_{p s}$ is in $\mathrm{keV}$ and $v_{s w}$ is in $\mathrm{km} / \mathrm{sec}$. The authors also determined that the geosynchronous midnight temperature obeyed

$$
T_{\text {geo }}=2.17+0.0223 v_{s w}
$$

with correlation coefficients of $R=0.51$ and $R=0.56$, respectively. In addition, the magnetotail neutral sheet density (in $1 / \mathrm{cm}^{3}$ ) obeyed

$$
n_{p s}=0.0785 n_{s w}^{0.62}
$$

and the geosynchronous midnight density obeyed

$$
n_{\text {geo }}=0.292 n_{s w}^{0.49}
$$


with correlation coefficients of $R=0.74$ and $R=0.67$, respectively. These simple dependencies on time-shifted solar wind parameters have been exploited for use in inner magnetospheric simulations by many authors, but this model has important limitations. Specifically, the lack of spacecraft coverage in the region between geosynchronous orbit and $17.5 R_{E}$ severely limits the ability to use this model for inner magnetospheric boundary conditions without making further assumptions. This is the case because most kinetic inner magne-

tospheric numerical models, including CRCM, have outer boundaries between 8-12 $R_{E} \cdot{ }^{18}$ In addition, this statistical study did not include any variation in MLT, which also limits the models' effectiveness in providing accurate boundary conditions to a numerical model, since cross-tail variation in plasma sheet properties is well-documented. ${ }^{1,2,19}$ Finally, this model's usefulness is also limited by the small data sets. The ISEE- 2 and -3 data set included 2 months of data approximately 8-9 months prior to the solar cycle 21 maximum (December 1979), and the LANL/IMP-8/WIND data set included 3 months of data approximately 2.5 years before the end (minimum), and 3.5 years after the maximum, of solar cycle 22. Due to the highly varied solar activity between the two data sets, it is important to note that the plasma sheet measurements were made under very different conditions than the geosynchronous measurements. Nonetheless, this model has proved to be very useful in computational studies ${ }^{20-23}$ although more recent statistical models have been developed using a much larger data set over continuous changes in solar activity.

\subsubsection{Tsyganenko \& Mukai Model}

Tsyganenko and $\mathrm{Mukai}^{24}$ made significant improvements over prior statistical models by using 3.5 years of Geotail ${ }^{25}$ data between November 1994 and April 1998. Observations in the central plasma sheet were made with Geotail's Low-Energy-Plasma (LEP) ${ }^{26}$ and magnetic 


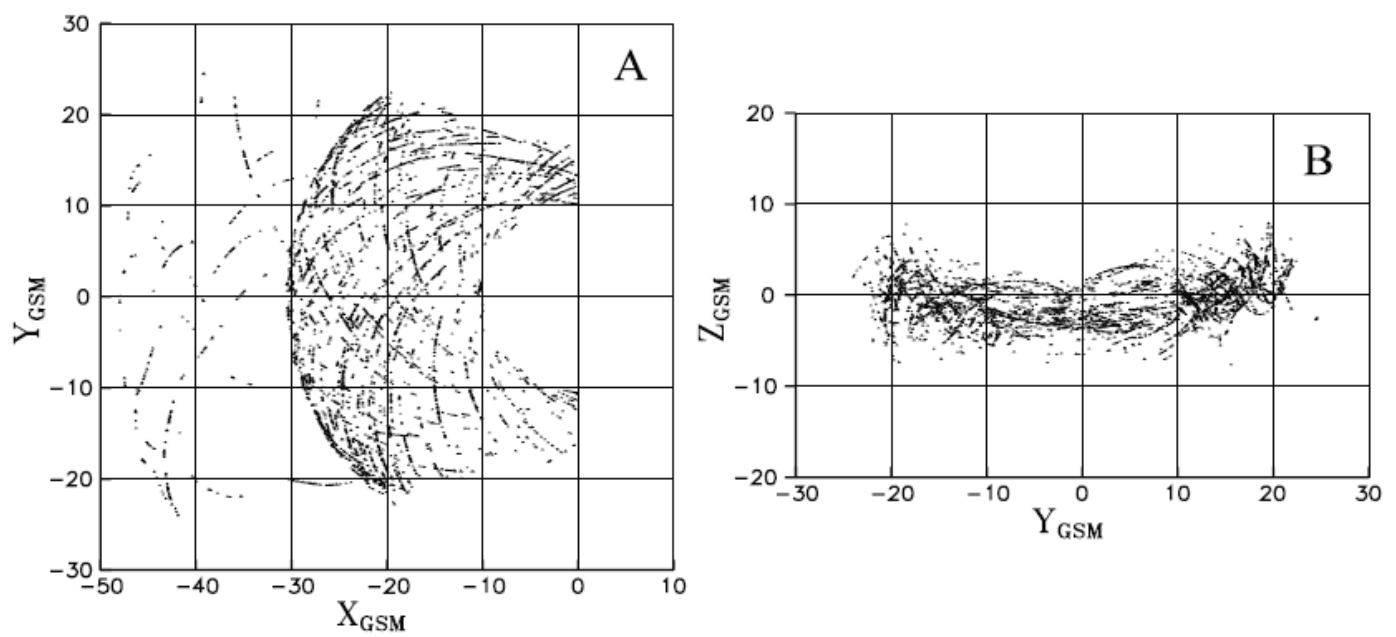

Figure 3.2: Distribution of the central plasma sheet crossings in the modeling data set: (a) projected on the equatorial (GSM XY) plane, and (b) in the cross-tail (GSM YZ) plane. Taken from Tsyganenko and Mukai. ${ }^{24}$

field $^{27}$ instruments, while concurrent solar wind and IMF observations were made with the IMP-8 and WIND spacecraft. Tsyganenko and Mukai used the Geotail instrument data to perform multivariate optimizations to determine the 2-D distributions of central plasma sheet parameters as functions of solar wind and interplanetary magnetic field parameters. The Geotail spacecraft covered radial distances of $10-50 R_{E}$ as well as a large range of nightside MLT; Figure 3.2 illustrates the data obtained for all central plasma sheet crossings which were used for their statistical analysis. Although the spacecraft coverage area does not include geosynchronous orbit, Geotail sampled the inner plasma sheet at near-Earth radial distances not included in the Borovsky et al. ${ }^{11}$ analysis.

The LEP data set used in their study covered the 0-40 keV energy range. The authors note that excluding $>40 \mathrm{keV}$ ions may underestimate temperature moments, but not by more than $5 \%$ if the calculated temperature is less than $8 \mathrm{keV}$. Upon testing multiple analytic functions to represent plasma sheet dependence upon solar wind parameters, the authors 
adopted a central plasma sheet temperature dependence of the form

$$
\begin{gathered}
T_{p s}=A_{1} v_{s w}^{*}+A_{2} B_{N}^{*}+A_{3} B_{S}^{*}+A_{4} \exp \left[-\left(A_{9} v_{s w}^{A_{15}}+A_{10} B_{N}^{*}+A_{11} B_{S}^{*}\right)\left(\rho^{*}-1\right)\right] \\
{\left[A_{5} v_{s w}^{*}+A_{6} B_{N}^{*}+A_{7} B_{S}^{*}+A_{8} \exp \left[-\left(A_{12} v_{s w}^{* A_{16}}+A_{13} B_{N}^{*}+A_{14} B_{S}^{*}\right)\left(\rho^{*}-1\right)\right]\right] \sin ^{2} \phi}
\end{gathered}
$$

where the values and errors for the coefficients $A_{k}$ may be obtained from the original publication. To get a sense of the statistical deviation in the temperature model, note that the correlation coefficient associated with this choice (equation 3.8) was $R=0.71$, with the average root-mean-square (RMS) temperature and RMS deviation being $3.79 \mathrm{keV}$ and $1.42 \mathrm{keV}$, respectively. Each of the 16 coefficients in the temperature equation has an associated error estimate calculated from the fitting procedure, also presented in their Table 1. Here $B_{N}^{*}$ $\left(B_{S}^{*}\right)$ is the normalized value of the northward (southward) component of the IMF, $v_{s w}^{*}$ is the normalized solar wind speed and $\rho^{*}$ is the normalized radial distance from Earth. $B_{N}^{*}$ and $B_{S}^{*}$ are both defined to be positive, i.e., $B_{N}=B_{Z} H\left(B_{Z}\right)$ and $B_{S}=-B_{Z} H\left(-B_{Z}\right)$ where $H$ is the Heaviside operator. ${ }^{28}$ The normalization factors are $5 \mathrm{nT}$ for $B_{N}$ and $B_{S}, 500 \mathrm{~km} / \mathrm{sec}$ for $v_{s w}$ and $10 R_{E}$ for $\rho$. The variable $\phi=-\tan ^{-1}\left(Y_{\mathrm{GSM}} / X_{\mathrm{GSM}}\right)$ is the angular deviation from midnight; positive $\phi$ tends toward dusk and negative $\phi$ tends dawnward. Note that the $\sim \sin ^{2} \phi$ dependence in equation 3.8 produces temperatures that are dusk-dawn symmetric about the midnight meridian. The authors' choice of mathematical forms for the fits permitted dawn-dusk asymmetries, but no such statistically significant asymmetry emerged from their analysis. The authors reported that a possible dawn-dusk asymmetry was observed within $\sim 1 R_{E}$ of the inner boundary $\left(\rho=10 R_{E}\right.$ ), but due to relatively sparse Geotail coverage and the level of temperature fluctuations in this region, the dawn-dusk asymmetry could 
not be reported with certainty.

Using the same set of central plasma sheet data, Tsyganenko and Mukai found a corresponding formula for the plasma sheet density $n_{p s}$ of the form

$$
\begin{aligned}
n_{p s}= & \left(A_{1}+A_{2} n_{s w}^{*^{A_{10}}}+A_{3} B_{N}^{*}+A_{4} v_{s w}^{*} B_{S}^{*}\right) \rho^{*^{A_{8}}} \\
& +\left(A_{5} n_{s w}^{*^{A_{11}}}+A_{6} B_{N}^{*}+A_{7} v_{s w}^{*} B_{S}^{*}\right) \rho^{*^{A_{9}}} \sin ^{2} \phi
\end{aligned}
$$

where these coefficients $A_{k}$ are not the same as the ones used equation 3.8. Again, the values and errors for each of these coefficients may be obtained from the Tsyganenko and Mukai report. The correlation coefficient reported by the authors was $R=0.57$. The normalization factor for $n_{s w}$ is $10 \mathrm{~cm}^{-3}$.

The time interval used in this analysis (November 1994 - April 1998) covers the late declining phase of solar cycle 22 through the beginning of increasing activity during solar cycle 23, and as such is not completely representative of other solar cycles or solar maximum. The TM plasma sheet model is only valid for the following solar wind conditions: $-5<B_{z}<+5$ $\mathrm{nT}, 300<v_{s w}<600 \mathrm{~km} / \mathrm{s}, 5<n_{s w}<20 \mathrm{~cm}^{-3}$. It is well-known that during active geomagnetic periods, the solar wind parameters at Earth frequently fall outside of these ranges. The authors' thorough statistical analysis has, however, supplied the space physics community with the first 2-D empirical model for determining average plasma sheet parameters from solar wind and IMF data. The results are of significant importance for inner magnetospheric modeling, especially since the near-Earth coverage was not included in prior data sets.

Although the data sets used in the Borovsky et al. and Tsyganenko and Mukai studies varied in coverage areas, solar cycle phase, and valid parameter regimes, a comparison be- 


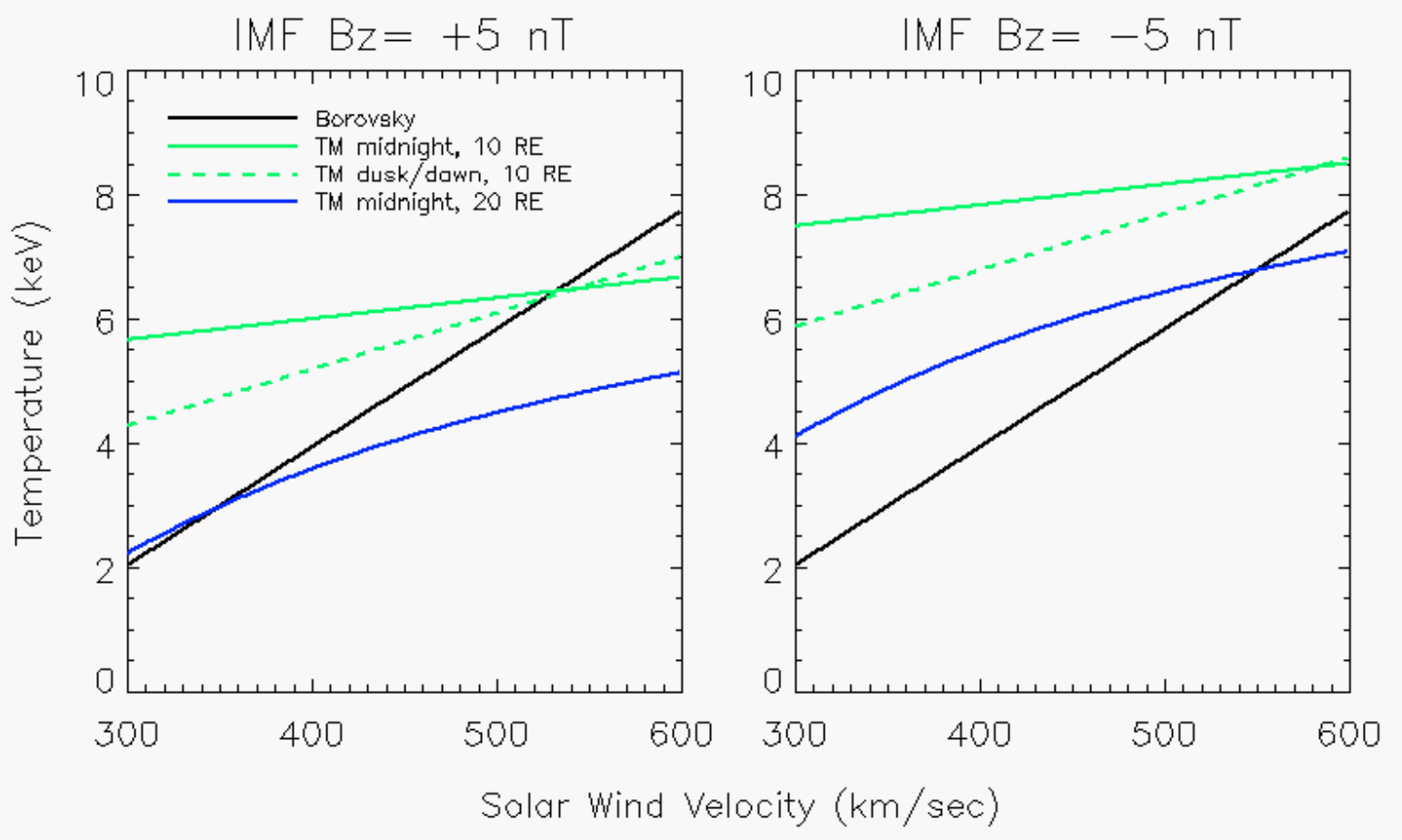

Figure 3.3: Comparison of plasma sheet ion temperatures at $X_{\mathrm{GSM}}=-20 R_{E}, Y_{\mathrm{GSM}}=0$ $R_{E}$ generated from the Borovsky et al. model (black) and the Tsyganenko and Mukai model (blue). The solid (dashed) green line shows TM temperatures at $10 R_{E}$ along the midnight (dawn or dusk) meridian. The northward (southward) IMF case is shown on the left (right).

tween these two models is in order. A comprehensive comparison cannot easily be made, as the functional form of the TM temperature model (equation 3.8) is simultaneously sensitive to multiple solar wind parameters and spatial location. Figure 3.3 shows the fundamental comparison of model temperatures at $20 R_{E}$ on the midnight meridian, where the TM model data is shown by the solid blue line, and the Borovsky et al. model data is shown in black. This comparison is done for the test cases of northward (left) and southward (right) IMF, each with 5 nT magnitude. The solid green lines show the TM model temperatures at 10 $R_{E}$ on the midnight meridian (the inner boundary of the TM model), and the dashed green line shows TM temperatures at $10 R_{E}$ on the dusk or dawn meridians. For northward IMF 
(left), the Borovsky et al. model predicts a hotter plasma sheet at $20 R_{E}$ than the TM model for nearly all solar wind speeds, while the TM model produces higher temperatures at this location during intervals of southward IMF (right). In fact, the TM model produces higher temperatures everywhere for southward IMF than for northward IMF, while the Borovsky et al. model temperature depends solely on the solar wind speed and thus has no IMF dependence. Recall that the Borovsky et al. plasma sheet data set was obtained near solar maximum, while the TM data set covered solar minimum, which certainly results in a discrepancy between the two models.

\subsubsection{Ionospheric Measurements}

Wing and $\mathrm{Newell}^{29}$ demonstrated that ionospheric observations made by a collection of polarorbiting satellites can be mapped back to the magnetotail plasma sheet, and showed that measurements taken in this way are in good agreement with average plasma sheet properties found from statistical studies based on in situ observations.

In their study the authors used ion particle measurements from electrostatic analyzers in the Special Sensor for Precipitating Particles, version 4 (SSJ4) instrument suites onboard DMSP satellites. The electrostatic analyzers detect ions with energies of $3-30 \mathrm{keV} .{ }^{30}$ The satellite orbits were polar configurations at $835 \mathrm{~km}$ altitude, and the data set covered the

full year of 1992, corresponding to an interval immediately following solar maximum of cycle 22. The authors took statistical averages of ion pressure, density and temperature over the full one-year time interval, thus giving an average response based on the solar wind conditions. The Tsyganenko 1989 (T89) ${ }^{31}$ magnetic field model was used in conjunction with corrections developed by Sergeev and Gvozdevsky ${ }^{32}$ to map ionospheric moments back to the equatorial magnetotail, the morphology of which is controlled by solar wind parameters and 
magnetospheric activity indices. ${ }^{29}$

In the original study the authors binned the inferred plasma sheet properties by the latitude of the ion isotropy boundary (DMSP b2i boundary), ${ }^{33}$ a proxy for the stretching of the near-Earth magnetotail, and thus also a proxy for geomagnetic activity level. In a later study, Wing and Newell ${ }^{34}$ performed a complementary analysis by binning the same set of 1992 data by the north-south orientation of the IMF, as well as by b2i $>64^{\circ}$, again producing plasma sheet moments consistent with in situ observations. ${ }^{1,10,35,36}$ Later, Wing et $a l .{ }^{37}$ expanded the analysis of the 1992 year and reported the detection of significant dawn-dusk asymmetries in plasma sheet temperature, density and pressure for the same time interval.

The techniques presented in these studies improve upon in situ based models in a variety of ways. First, the orbital period of each DMSP spacecraft is approximately 100 minutes, and each spacecraft samples a wide range of $L$-shells during each orbit. Thus, using ionospheric satellites provides greater spatial coverage, given a magnetic field model, in a much shorter time than in situ satellites can manage. Second, the SSJ4 instrument suite samples the 19-point ion energy spectrum once per second, which in effect provides excellent spatial resolution. The aforementioned Wing and Newell and Wing et al. reports fix the spatial resolution at $1 \times 1 R_{E}^{2}$ in the equatorial plane.

\subsection{Determination of Ion Temperature Boundary Con- ditions from ENA maps}

Empirical or statistical plasma sheet models, such as those discussed in sections 3.2.1-3.2.3, are extraordinarily important from a modeling perspective, but important limitations exist 
and must be considered. Empirical models built up from in situ measurements present an average response in the statistical sense, because the samples are taken over such a long time interval. Event-specific or transient features are not represented well in such data sets because they are effectively averaged over during statistical analysis.

Spatiotemporally resolved measurements from a specific event can also be supplied as boundary conditions to numerical simulations, but this requires either multi-point in situ measurements or remote imaging. Multi-point measurements taken from collections of MPA satellites have been used by Liemohn et al. ${ }^{38}$ and Zaharia et al. ${ }^{39,40}$ as plasma sheet inputs to RAM and RCM simulations for the purposes of (1) discerning the relative importance of storm-time ring current loss mechanisms and (2) modeling self-consistent, anisotropic field-plasma feedback and thus producing equilibrium snapshots of the storm-time inner magnetosphere, respectively. Chen et al. ${ }^{41}$ used 20-minute Geotail averages of proton density and temperature to examine how MLT dependence affects the preconditioning and formation of the ring current. Since Geotail only took measurements beyond $\approx 8 R_{E}$, Chen et al. employed a mapping technique based on an empirical magnetic field model to calculate particle drifts and thus prescribe boundary conditions at geosynchronous orbit. All of the above studies use in situ based boundary conditions that are supplied to their respective numerical models at geosynchronous orbit. As an alternative, remote observations made through charge exchange imaging can provide more complete coverage of the magnetosphere and thus provide temporally-resolved boundary conditions at any desired locations in space. A discussion of how energetic neutral atom (ENA) images may be used to determine ion temperature boundary conditions follows.

Consider ENA emission collected by a specific instrument pixel, that is, ENA emission collected along a particular line of sight subtending a solid angle $\Omega_{s}$. Let $S(\vec{x}, \vec{v}) d \vec{v}$ be the 
birth rate of neutral atoms at position $\vec{x}$ with velocity $\vec{v}$ within the velocity element $d \vec{v}$. This birth rate includes neutral atoms generated by ionization and charge exchange; neutral atoms may be ionized through collisions with electrons or protons, and charge exchange may only occur through proton-neutral collisions. Let $\sigma_{e i}, \sigma_{p i}$, and $\sigma_{c x}$ be the cross sections of electron ionization, proton ionization, and charge exchange respectively.

The instrument neutral collection rate $[\# / \mathrm{sec}]$ in the energy element $d E$ is given by

$$
F(E) d E=v^{2} d v \Omega_{s} \int e^{-\int_{z}^{a} \alpha(l) d l} S(\vec{x}, \vec{v}) d \vec{x}
$$

where the exponential factor $e^{-\int_{z}^{a} \alpha(l) d l}$ accounts for attenuation of neutral flux due to the various collisional processes occurring along the neutral's path from the birth location to the detector ( $a$, the upper integration limit), where the line-of-sight direction is along $l$. The attenuation coefficient $\alpha(l)$ is given by

$$
\alpha(l)=\frac{1}{v}\left(\left\langle\sigma_{e i} v_{e}\right\rangle_{v} n_{e}+\left\langle\sigma_{p i} v_{i}\right\rangle_{v} n_{i}+\left\langle\sigma_{c x} v_{i}\right\rangle_{v} n_{i}\right)
$$

Considering the extremely low density of ions and electrons with high enough energies to ionize and liberate electrons from impacted neutral atoms, charge exchange collisions dominate this attenuation term. Thus, the production rate of neutrals due to charge exchange collisions between the neutral hydrogen background and an incident ion with velocity $\vec{v}_{i}$ is given by

$$
\int d^{3} v_{H} \sigma_{c x}\left(\left|\vec{v}_{i}-\vec{v}_{H}\right|\right)\left|\vec{v}_{i}-\vec{v}_{H}\right| f_{H}\left(\vec{x}, \vec{v}_{H}\right)
$$

which gives a total neutral birth rate of 


$$
S\left(\vec{x}, \vec{v}_{i}\right)=f_{i}\left(\vec{x}, \vec{v}_{i}\right) \int d^{3} v_{H} \sigma_{c x}\left(\left|\vec{v}_{i}-\vec{v}_{H}\right|\right)\left|\vec{v}_{i}-\vec{v}_{H}\right| f_{H}\left(\vec{x}, \vec{v}_{H}\right)
$$

where $f_{H}$ and $f_{i}$ are the neutral hydrogen and ion distribution functions respectively. If, prior to the collision, the ion is considerably more energetic than the neutral atom, equation 3.13 simplifies considerably because $\left|\vec{v}_{i}-\vec{v}_{H}\right| \approx v_{i}$ and therefore equation 3.13 becomes

$$
S\left(\vec{x}, \vec{v}_{i}\right)=f_{i}\left(\vec{x}, \vec{v}_{i}\right) \sigma_{c x}\left(v_{i}\right) v_{i} \int d^{3} v_{H} f_{H}\left(\vec{x}, \vec{v}_{H}\right)
$$

and the integral is easily identifiable as the zeroth moment of the neutral distribution function, known as the neutral density $n_{H}(\vec{x})$. Thus

$$
S\left(\vec{x}, \vec{v}_{i}\right)=f_{i}\left(\vec{x}, \vec{v}_{i}\right) \sigma_{c x}\left(v_{i}\right) v_{i} n_{H}(\vec{x})
$$

Noting that upon charge exchange, the incident ion retains its energy upon becoming a neutral atom, we may now rewrite equation 3.10 for the total neutral collection rate in the differential energy element $d E$ as

$$
F(E) d E=v^{2} \Omega_{s} d v \sigma_{c x}(E) v \int d^{3} x_{H} f_{i}(\vec{x}, \vec{v}) n_{H}(\vec{x}) e^{-\int_{z}^{a} \alpha(l) d l}
$$

The spatial integral is bounded in the $\hat{x}, \hat{y}$ directions by the spatial extent of an instrument pixel. Given sound spatial resolution, one may assume that the neutral emission is uniform in $\hat{x}$ and $\hat{y}$ across the volume of plasma contributing to the measurements made by a single pixel and thus $d x d y=\Delta x \Delta y=\Delta A$. Also noting that $v^{3} d v=2 E d E / m^{2}$ gives

$$
F(E) d E=\frac{2 E d E}{m^{2}} \Delta A \sigma_{c x}(E) \Omega_{s} \int d z f_{i}(z, \vec{v}) n_{H}(z) e^{-\int_{z}^{a} \alpha(l) d l}
$$


A suitable ion distribution function $f_{i}(\vec{v})$ may now be assumed and substituted into the equation 3.17 integrand. In the case of a Maxwellian description, i.e.,

$$
f_{i}(z, \vec{v})=\frac{n_{i}(z) m_{i}^{3 / 2}}{\left(2 \pi k_{B} T_{i}(z)\right)^{3 / 2}} e^{-m v^{2} / 2 k_{B} T_{i}(z)}
$$

which is readily inferred from equation 3.1, one may infer the primary contribution to the spatial integral in equation 3.17 by differentiating the integrand with respect to $z$ and setting the result equal to zero. This yields

$$
\frac{1}{n_{i}(z)} \frac{d n_{i}(z)}{d z}+\frac{1}{n_{H}(z)} \frac{d n_{H}(z)}{d z}-\frac{3}{2 T_{i}(z)} \frac{d T_{i}(z)}{d z}+\frac{E}{k_{B} T_{i}^{2}(z)} \frac{d T_{i}(z)}{d z}+\alpha=0
$$

The high energy portion of the energy spectrum, $F(E)$, is dominated by the hottest region along that line of sight, ${ }^{42,43}$ i.e., where $d T_{i}(z) / d z=0$ and $T_{i}(z)$ is a maximum. Let the $z$ value corresponding to the hottest region be $z=z^{*}$. The neutral flux expression from equation 3.17 then reduces to

$$
F(E) d E=E d E \Delta A \xi \sigma_{c x}(E) \Omega_{s} \frac{n_{i}\left(z^{*}\right) n_{H}\left(z^{*}\right)}{\sqrt{2 m_{i}}\left(\pi k_{B} T_{i}\left(z^{*}\right)\right)^{3 / 2}} e^{-E / k_{B} T_{i}\left(z^{*}\right)} e^{-\int_{z^{*}}^{a} \alpha(l) d l}
$$

where $\xi$ is the characteristic thickness of the hottest region along the line of sight. In low density plasmas, the attenuation term $e^{\int_{z^{*}}^{a} \alpha(l) d l} \approx 1$, and we use $C=\xi \Delta A \Omega_{s}$ to denote a purely geometric factor which accounts for the instrument viewing perspective. This implies that the ion and neutral density derivatives are also approximately zero at $z=z^{*}$. Thus, 


$$
\frac{F(E) d E}{C \sigma_{c x}(E) E d E} \approx \frac{n_{i}\left(z^{*}\right) n_{H}\left(z^{*}\right)}{\sqrt{2 m_{i}}\left(\pi k_{B} T_{i}\left(z^{*}\right)\right)^{3 / 2}} e^{-E / k_{B} T_{i}\left(z^{*}\right)}
$$

and

$$
\ln \left(\frac{F(E) d E}{C \sigma_{c x}(E) E d E}\right) \approx \ln \left(\frac{n_{i}\left(z^{*}\right) n_{H}\left(z^{*}\right)}{\sqrt{2 m_{i}}\left(\pi k_{B} T_{i}\left(z^{*}\right)\right)^{3 / 2}}\right)-\frac{E}{k_{B} T_{i}\left(z^{*}\right)}
$$

The peak ion temperature, $T_{i}\left(z^{*}\right)$ along the line of sight may be determined by fits against the 'corrected' observed energy spectra given on the LHS of equation 3.22. Temperatures calculated in this manner are inherently isotropic i.e., parallel and perpendicular temperatures are assumed equal. ${ }^{42}$ Note that fluxes in the 1-32 keV energy range are used for such temperature fits when using TWINS ENA data. It has been found empirically within our research group that the quality of the fits suffer when higher energy bins are included, giving artificially high temperatures. In addition, this energy range is consistent with the $0-40 \mathrm{keV}$ energy range used for the TM model. Temperatures are calculated in this manner for each spatial pixel, where each pixel corresponds to a single instrument look direction. A global map of ENA fluxes as observed from the spacecraft location is constructed, after collecting the ENA flux over the desired number of instrument actuator sweeps. Upon applying the algorithm discussed in this section, a global map of time-integrated ENA flux is obtained. Examples of these 'fisheye' maps viewed from the spacecraft vantage point can be found in Figure 2 of Keesee et al. ${ }^{44}$ Corresponding ENA fluxes are then determined in the magnetic equatorial plane by mapping along the line-of-sight of each instrument pixel, given the time-dependent spacecraft location and look direction. A cartoon depiction of the TWINS instruments taking LOS-integrated measurements is shown in Figure 3.4; there the TWINS 2 LOS is shown to pass through both optically thick and optically thin regions of the inner 
magnetosphere. Particles precipitating into the relatively dense atmosphere and ionosphere generate low altitude emission (LAE), whereas emission from trapped ring current ions occurs in a less dense, optically thin region. The GSM equatorial (x-y) plane is divided into 0.5 $R_{E} \times 0.5 R_{E}$ pixels. The areas of intersection between the field of view (FOV) of each instrument pixel and each equatorial pixel are calculated. The ENA flux multiplied by the square of the line-of-sight magnitude is subdivided into the equatorial pixels which it intersects; the overlapping area determines the fraction which is placed into a particular equatorial bin. A given equatorial bin may share intersections with multiple instrument pixel FOVs; the final energy-resolved ENA fluxes in each equatorial pixel are taken as averages over the contributing instrument FOVs. An example projection of an IMAGE ENA instrument pixel is shown in Figure 3.5.

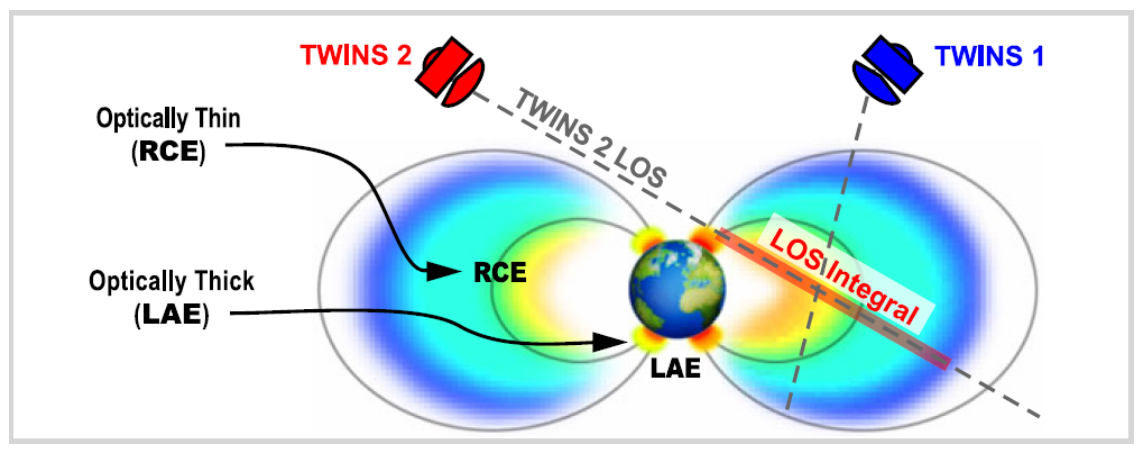

Figure 3.4: Cartoon illustration of the TWINS instrument FOVs from high altitude. Lines of sight from arbitrary instrument pixels are shown with dashed lines. From Goldstein and McComas. ${ }^{45}$

The fundamental time step for advancing distribution functions and boundary conditions in CRCM is 10 seconds, and thus temperature boundary conditions for each CRCM boundary cell are linearly interpolated from one ENA-derived temperature map to the next. This interpolation is used to ensure smooth changes in boundary conditions and thereby prevent artificially steep phase space gradients, which drive the simulations unstable. To assign 


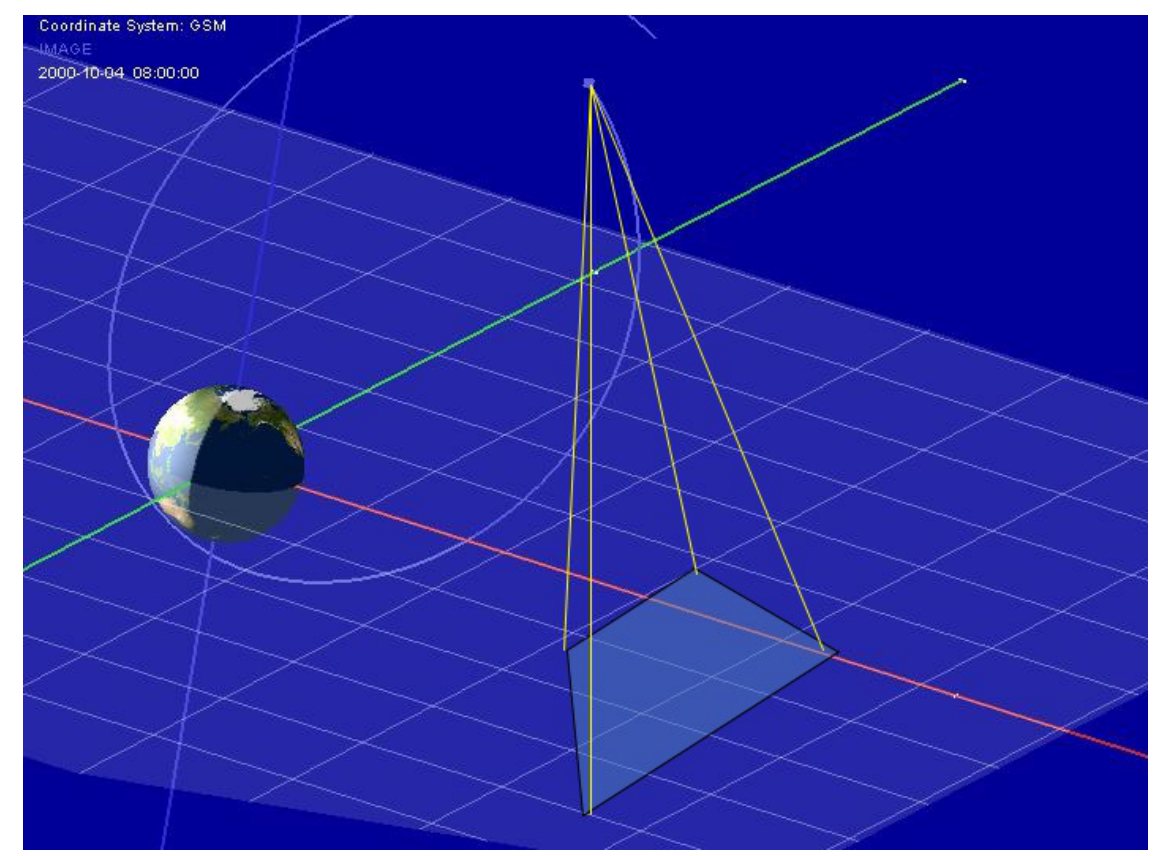

Figure 3.5: Illustration of a single IMAGE ENA instrument pixel is projected into the GSM equatorial plane. Figure courtesy of Amy Keesee.

ion temperatures in bins where no temperature data is available (outside the instrument field of view), we first take available data and mirror about the $Y_{\mathrm{GSM}}=0$ axis, ensuring that existing data is not overwritten. Although dusk-dawn asymmetries are prolific in the inner magnetosphere, this mirroring method is consistent with the TM method, which is arguably the most comprehensive statistical plasma sheet model currently available. Any remaining pixels which have no ion temperature defined are assigned the mean temperature of the full map in the region $X_{\mathrm{GSM}}, Y_{\mathrm{GSM}}=[-20,20] R_{E}$. Examples of 'before and after' temperature maps are shown in chapter 4.

Instead of assuming a Maxwellian distribution in equation 3.18, a kappa distribution (equation 3.3) could be chosen. The same set of assumptions would be valid, giving the following result in place of equation 3.21: 


$$
\frac{F(E) d E}{C \sigma_{c x}(E) E d E} \approx \frac{\Gamma(\kappa+1) \sqrt{2 m_{i}}}{(2 \kappa-3)^{3 / 2} \Gamma\left(\frac{\kappa-1}{2}\right)} \frac{n_{i}\left(z^{*}\right) n_{H}\left(z^{*}\right)}{\left(\pi k_{B} T_{i}\left(z^{*}\right)\right)^{3 / 2}}\left(1+\frac{2 E}{(2 \kappa-3) k_{B} T_{i}\left(z^{*}\right)}\right)^{-1-\kappa}
$$

Thus, for a kappa distribution

$$
\begin{aligned}
\ln \left(\frac{F(E) d E}{C \sigma_{c x}(E) E d E}\right) \approx \ln & \left(\frac{\Gamma(\kappa+1) \sqrt{2 m_{i}}}{(2 \kappa-3)^{3 / 2} \Gamma\left(\frac{\kappa-1}{2}\right)} \frac{n_{i}\left(z^{*}\right) n_{H}\left(z^{*}\right)}{\left(\pi k_{B} T_{i}\left(z^{*}\right)\right)^{3 / 2}}\right) \\
& -(1+\kappa) \ln \left(1+\frac{2 E}{(2 \kappa-3) k_{B} T_{i}\left(z^{*}\right)}\right)
\end{aligned}
$$

provides the corrected energy spectrum on the LHS, and fits can be used to determine the peak ion temperature $T_{i}\left(z^{*}\right)$. Note that this form requires the determination of the $\kappa$ free parameter as well, which will potentially introduce additional uncertainties into the fitting process. This is a well-known problem in nonlinear fitting algorithms. Alternatively, a user may opt to specify a value of $\kappa$ and then perform the fit to obtain $T_{i}\left(z^{*}\right)$, but such a choice should not be made arbitrarily.

The techniques discussed here may be applied to ENA measurements taken in optically thin environments, such as outside of the plasmapause in Earth's magnetosphere. Several efforts have been made to validate temperatures computed in this way with in situ data. Specifically, Scime et al. ${ }^{46}$ showed that ion temperatures calculated using ENA image processing techniques were in nearly perfect agreement with storm-time measurements taken by MPA spacecraft at geosynchronous orbit. Keesee et al. ${ }^{47}$ performed a comparison of MENA temperatures with in situ temperatures obtained from MPA and Geotail, and also performed a comparison with predictions from the Borovsky et al. empirical model. In addition, several 
authors have applied this technique to ENA images produced using the imagers aboard the two TWINS spacecraft. ${ }^{44,48-50}$

Using these event-specific boundary conditions in a ring current model provides a number of advantages over statistical models. One drawback of using statistical models is that these models have limitations on the parameter regimes in which the models are valid (see Section 3.2.2). In addition, the TM model is valid only at distances between 10 and 50 $R_{E}$, which leaves an important observational gap between geosynchronous orbit and $10 R_{E}$. Finally, the TM model is also solely based on data from solar cycles 22 and 23, and thus this plasma sheet model is not completely appropriate for other cycles or for conditions during solar maximum. Thus, when performing numerical simulations to study the stormtime coupling between the solar wind, the plasma sheet, and the inner magnetosphere, it is necessary to find a more appropriate description of the plasma at the outer boundary. Using boundary conditions that are determined using ENA measurements circumvents the formidable statistical limitations, and implementation of these new boundary conditions is not particularly challenging. In addition, these temperature maps serve to bridge the important spatial gap between geosynchronous orbit and $10 R_{E}$, a region where in situ data is sparse.

In the next chapter, results from CRCM simulations using these boundary conditions are presented in comparison with TWINS and THEMIS data. 


\section{References}

[1] W. Baumjohann, G. Paschmann, and C. A. Cattell, "Average plasma properties in the central plasma sheet," Journal of Geophysical Research, vol. 94, no. A6, pp. 6597-6606, 1989.

[2] V. Angelopoulos, C. F. Kennel, F. V. Coroniti, R. Pellat, H. E. Spence, M. G. Kivelson, R. J. Walker, W. Baumjohann, W. C. Feldman, J. T. Gosling, and C. T. Russell, "Characteristics of ion flow in the quiet state of the inner plasma sheet," Geophysical Research Letters, vol. 20, no. 16, pp. 1711-1714, 1993.

[3] R. W. Hockney and J. W. Eastwood, Computer Simulation Using Particles. IOP Publishing, 1988.

[4] J. Yang, F. R. Toffoletto, R. A. Wolf, S. Sazykin, R. W. Spiro, P. C. Brandt, M. G. Henderson, and H. U. Frey, "Rice convection model simulation of the 18 april 2002 sawtooth event and evidence for interchange instability," Journal of Geophysical Research, vol. 113, no. A11214, pp. 1-15, 2008.

[5] M. W. Liemohn, J. U. Kozyra, A. J. Ridley, M. F. Thomsen, M. G. Henderson, P. C. Brandt, and D. G. Mitchell, "Modeling the ring current response to a sawtooth oscillation event," Journal of Atmospheric and Solar-Terrestrial Physics, vol. 69, pp. 67-76, 2007.

[6] W. Baumjohann and R. A. Treumann, Basic Space Plasma Physics. Imperial College Press, 1997.

[7] M.-B. Kallenrode, Space Physics: An Introduction to Plasmas and Particles in the Heliosphere and Magnetospheres. Springer, 3rd ed., 2004. 
[8] V. Pierrard and M. Lazar, "Kappa distributions: theory and applications in space plasmas," Solar Physics, vol. 267, no. 1, pp. 153-174, 2010.

[9] C. K. Goertz and W. Baumjohann, "On the thermodynamics of the plasma sheet," Journal of Geophysical Research, vol. 96, no. A12, pp. 20991-20998, 1991.

[10] W. Lennartsson and E. G. Shelley, "Survey of 0.1- to 16-kev/e plasma sheet ion composition," Journal of Geophysical Research, vol. 91, no. A3, pp. 3061-3076, 1986.

[11] J. E. Borovsky, M. F. Thomsen, and R. C. Elphic, "The driving of the plasma sheet by the solar wind," Journal of Geophysical Research, vol. 107, no. A8, pp. 17617-17639, 1998.

[12] S. J. Bame, J. R. Asbridge, H. E. Felthauser, J. P. Glore, G. Paschmann, P. Hemmerlich, K. Lehmann, and H. Rosenbauer, "Isee-1 and isee-2 fast plasma experiment and the isee-1 solar wind experiment," IEEE Transactions on Geoscience Electronics, vol. 16, pp. 216-220, 1978.

[13] C. T. Russell, "The isee 1 and 2 fluxgate magnetometers," IEEE Transactions on Geoscience Electronics, vol. 16, p. 239, 1978.

[14] S. J. Bame, J. R. Asbridge, H. E. Felthauser, J. P. Glore, H. L. Hawk, and J. Chavez, "Isee-c solar wind plasma experiment," IEEE Transactions on Geoscience Electronics, vol. 16, p. 160, 1978.

[15] A. M. A. Frandsen, B. V. Conner, J. van Amersfoort, and E. J. Smith, "The isee-c vector helium magnetometer," IEEE Transactions on Geoscience Electronics, vol. 16, p. 195, 1978. 
[16] S. J. Bame, D. J. McComas, M. F. Thomsen, B. L. Baraclough, R. C. Elphic, J. P. Glore, J. T. Gosling, J. C. Chavez, E. P. Evans, and F. J. Wymer, "Magnetospheric plasma analyzer for spacecraft with constrained resources," Review of Scientific Instruments, vol. 64, p. 1026, 1993.

[17] K. W. Ogilvie, D. J. Chornay, R. J. Fritzenreiter, F. Hunsaker, J. Keller, J. Lobell, G. Miller, J. D. Scudder, E. C. S. Jr., R. B. Torbert, D. Bodet, G. Needell, A. J. Lazarus, J. T. Steinberg, J. H. Tappan, A. Mavretic, and E. Gergin, "Swe, a comprehensive plasma instrument for the wind spacecraft," Space Science Reviews, vol. 71, p. 55, 1995.

[18] A. Glocer, M. Fok, X. Meng, G. Toth, N. Buzulukova, S. Chen, and K. Lin, "Crcm + bats-r-us two-way coupling," Journal of Geophysical Research, vol. 118, pp. 1635-1650, 2013.

[19] V. Angelopoulos, C. F. Kennel, F. V. Coroniti, R. Pellat, M. G. Kivelson, R. J. Walker, C. T. Russell, W. Baumjohann, W. C. Feldman, and J. T. Gosling, "Statistical characteristics of bursty bulk flow events," Journal of Geophysical Research, vol. 99, no. A11, pp. 21257-21280, 1994.

[20] T. W. Garner, R. A. Wolf, R. W. Spiro, W. J. Burke, B. G. Fejer, S. Sazykin, J. L. Roeder, and M. R. Hairston, "Magnetospheric electric fields and plasma sheet injection to low l-shells during the 4-5 june 1991 magnetic storm: Comparison between the rice convection model and observations," Journal of Geophysical Research, vol. 109, no. A02214, pp. 1-19, 2004.

[21] N. Buzulukova and V. Vovchenko, "Modeling of proton nose structures in the inner 
magnetosphere with a self-consistent electric field model," Journal of Atmospheric and Solar-Terrestrial Physics, vol. 70, no. 2-4, pp. 503-510, 2008.

[22] Y. Zheng, A. T. Y. Lui, M. C. Fok, B. J. Anderson, P. C. Brandt, and D. G. Mitchell, "Controlling factors of region 2 field-aligned current and its relationship to the ring current: Model results," Advances in Space Research, vol. 41, pp. 1234-1242, 2008.

[23] Y. Zheng, A. T. Y. Lui, and M. C. Fok, "Effects of plasma sheet properties on stormtime ring current," Journal of Geophysical Research, vol. 115, no. A08220, 2010.

[24] N. A. Tsyganenko and T. Mukai, "Tail plasma sheet models derived from geotail particle data," Journal of Geophysical Research, vol. 108, no. A3, p. 1136, 2003.

[25] A. Nishida, "The geotail mission," Geophysical Research Letters, vol. 21, no. 25, pp. 2871-2873, 1994.

[26] T. Mukai, S. Machida, Y. Saito, M. Hirahara, T. Terasawa, N. Kaya, T. Obara, M. Ejiri, and A. Nishida, "The low energy particle (lep) experiment onboard the geotail satellite," Journal of Geomagnetism and Geoelectricity, vol. 46, pp. 669-692, 1994.

[27] S. Kokubun, T. Yamamoto, M. H. Acuna, K. Hayashi, K. Shiokawa, and H. Kawano, "The geotail magnetic field experiment," Journal of Geomagnetism and Geoelectricity, vol. 46, pp. 7-21, 1994.

[28] M. Abramowitz and I. A. Stegun, eds., Handbook of Mathematical Functions With Formulas, Graphs, and Mathematical Tables, vol. 55 of Applied Mathematics Series. National Bureau of Standards, 1964. 
[29] S. Wing and P. T. Newell, "Central plasma sheet ion properties as inferred from ionospheric observations," Journal of Geophysical Research, vol. 103, no. A4, pp. 6785-6800, 1998.

[30] D. A. Hardy, L. K. Schmitt, M. S. Gussenhoven, F. J. Marshall, H. C. Yeh, T. L. Shumaker, A. Hube, and J. Pantazis, "Precipitating electron and ion detectors (ssj/4) for the block 5d/flights 6-10 dmsp satellites: Calibration and data presentation," Tech. Rep. AFGL-TR-84-0317, Air Force Geophys. Lab., Hanscom Air Force Base, 1984.

[31] N. A. Tsyganenko, "A magnetospheric magnetic field model with a warped tail current sheet," Planetary Space Sciences, vol. 37, pp. 5-20, 1989.

[32] V. A. Sergeev and B. B. Gvozdevsky, "Mt-index: A possible new index to characterize the magnetic configuration of magnetotail," Annales Geophysicae, vol. 13, pp. 1093$1103,1995$.

[33] P. T. Newell, Y. I. Feldstein, Y. I. Galperin, and C. I. Meng, "Morphology of nightside precipitation," Journal of Geophysical Research, vol. 101, pp. 10737-10748, 1996.

[34] S. Wing and P. T. Newell, "2d plasma sheet ion density and temperature profiles for northward and southward imf," Geophysical Research Letters, vol. 29, no. 9, p. 1307, 2002.

[35] T. Terasawa, M. Fujimoto, T. Mukai, I. Shinohara, Y. Saito, T. Yamamoto, S. Machida, S. Kokubun, A. J. Lazarus, J. T. Steinberg, and R. P. Lepping, "Solar wind control of density and temperature in the near-earth plasma sheet: Wind/geotail collaboration," Geophysical Research Letters, vol. 24, no. 8, pp. 935-938, 1997. 
[36] W. Lennartsson, “A scenario for solar wind penetration of earth's magnetic tail based on ion composition data from the isee 1 spacecraft," Journal of Geophysical Research, vol. 97, no. 19, p. 221, 1992.

[37] S. Wing, J. R. Johnson, P. T. Newell, and C. I. Meng, "Dawn-dusk asymmetries, ion spectra, and sources in the northward interplanetary magnetic field plasma sheet," Journal of Geophysical Research, vol. 110, no. A08205, 2005.

[38] M. W. Liemohn, J. U. Kozyra, V. K. Jordanova, G. V. Khazanov, M. F. Thomsen, and T. E. Cayton, "Analysis of early phase ring current recovery mechanisms during geomagnetic storms," Geophysical Research Letters, vol. 26, no. 18, pp. 2845-2848, 1999.

[39] S. Zaharia, M. F. Thomsen, J. Birn, M. H. Denton, V. K. Jordanova, and C. Z. Cheng, "Effect of storm-time plasma pressure on the magnetic field in the inner magnetosphere," Geophysical Research Letters, vol. 32, no. L03102, pp. 1-4, 2005.

[40] S. Zaharia, V. K. Jordanova, M. F. Thomsen, and G. D. Reeves, "Self-consistent modeling of magnetic fields and plasmas in the inner magnetosphere: Application to a geomagnetic storm," Journal of Geophysical Research, vol. 111, no. A11S14, pp. 1-14, 2006.

[41] M. C. Chen, C. P. Wang, M. Schulz, and L. R. Lyons, "Solar-wind influence on mlt dependence of plasma sheet conditions and their effects on storm time ring current formation," Geophysical Research Letters, vol. 34, p. L14112, 2007.

[42] I. H. Hutchinson, Principles of Plasma Diagnostics. Cambridge University Press, 1987.

[43] E. E. Scime and S. Hokin, "Design and calibration of a fast time resolution charge exchange analyzer," Review of Scientific Instruments, vol. 63, p. 4527, 1992. 
[44] A. M. Keesee, J. G. Elfritz, D. J. McComas, and E. E. Scime, "Inner magnetosphere convection and magnetotail structure of hot ions imaged by ena during a hss-driven storm," Journal of Geophysical Research, vol. 117, no. A00L06, pp. 1-9, 2012.

[45] J. Goldstein and D. J. McComas, "Five years of stereo magnetospheric imaging by twins," Space Science Reviews, vol. 180, pp. 39-70, 2013.

[46] E. E. Scime, A. M. Keesee, J. M. Jahn, J. L. Kline, C. J. Pollock, and M. Thomsen, "Remote ion temperature measurements of earth's magnetosphere: Medium energy neutral atom (mena) images," Geophysical Research Letters, vol. 29, no. 10, p. 1438, 2002.

[47] A. M. Keesee, E. E. Scime, and M. Moldwin, "Remote measurements of ion temperatures in the terrestrial magnetotail," Journal of Geophysical Research, vol. 113, no. A00A03, pp. 1-9, 2008.

[48] A. M. Keesee, N. Buzulukova, J. Goldstein, D. J. McComas, E. E. Scime, H. Spence, M. C. Fok, and K. Tallaksen, "Remote observations of ion temperatures in the quiet time magnetosphere," Geophysical Research Letters, vol. 38, no. L03104, pp. 1-5, 2011.

[49] A. M. Keesee, J. G. Elfritz, D. J. McComas, and E. E. Scime, "Superposed epoch analyses of ion temperatures during cme and cir/hss-driven storms," Journal of Atmospheric and Solar-Terrestrial Physics, vol. 115-116, pp. 67-78, 2013.

[50] J. G. Elfritz, A. M. Keesee, N. Buzulukova, M. C. Fok, and E. E. Scime, "First results using twins-derived ion temperature boundary conditions in crcm," Journal of Geophysical Research: Space Physics, vol. 119, 2014. 


\section{Chapter 4}

\section{Event Studies}

The importance of understanding magnetospheric dynamics during space weather events has been emphasized in Chapter 1. Studying the magnetospheric response during a specific interval of increased geomagnetic activity, ultimately driven by structures in the solar wind, is a key element of space physics research. After all, impulsive short-duration events such as coronal mass ejections (CMEs) are one of the biggest threats to space-based technology and infrastructure. In addition, high-speed streams (HSSs) pose space weather threats, and it is increasingly accepted that both types of geomagnetic storm drivers affect magnetospheric regions in unique ways. Event studies frequently serve as the key metric in space physics research, especially through the use of data-model comparisons. Typical event studies employ space-based and ground-based observations in conjunction with results from numerical simulations to probe the magnetospheric response during real events and improve the community's ability to forecast and nowcast space weather impacts.

In the following two sections of this chapter, simulations and observations of two separate geomagnetic storms are discussed. First, the 22 July 2009 high-speed stream-driven event is analyzed in Section 4.1, followed by a discussion of the 30 October 2013 CME-driven storm 
in Section 4.2. These two specific events were selected for analysis for a variety of reasons. First, TWINS observations are only available beyond 2008, constraining the selection pool to somewhat recent events. Second, it is advantageous to analyze a moderate CME-driven storm alongside an HSS-driven storm with similar geomagnetic effectiveness; such a study is intended to shed new light on how storm-time magnetospheric ion temperatures evolve in response to the two distinct solar wind driving mechanisms.

\subsection{The 22 July 2009 Event}

\subsubsection{Solar Wind Parameters}

On 22 July 2009 a southward oriented IMF reached Earth's magnetosphere and was followed by a high-speed stream (HSS). At the time, this was the most intense storm observed during the minimum of solar cycle 23. Following 2000 UT on 21 July 2009 a gradual decrease in the Sym-H index was observed, during which time the IMF $B_{z}$ was weak and southward. Near 0100 UT on 22 July, the southward IMF $B_{z}$ increased in magnitude, the solar wind density $n_{s w}$ increased, and the Sym-H index concurrently decreased. The main phase persisted until 0530 UT when the IMF turned northward. The northward turning resulted in a shortlived recovery from 0530-0715 UT. The IMF again turned southward from 0715-0900 UT resulting in a second Sym-H minimum. After 0900 UT, the recovery phase began. During the beginning of the recovery phase, the IMF was primarily northward, and the AL index indicates enhanced substorm activity. After 1130 UT, solar wind conditions remained steady for the duration of the recovery. Figure 4.1 shows Advanced Composition Explorer (ACE) data shifted to the bow shock nose and geomagnetic indices for the relevant time interval of our simulations; ACE data is obtained through the OMNIWeb interface. ${ }^{1}$ The Sym-H index 
indicates the storm phase, and the AU and AL indices show moderate substorm activity. The solar wind density $n_{s w}$ and speed $v_{s w}$ show clear high-speed stream signatures starting late in the main phase, ${ }^{2}$ and the IMF $B_{y}$ and $B_{z}$ both show storm-time reversals.

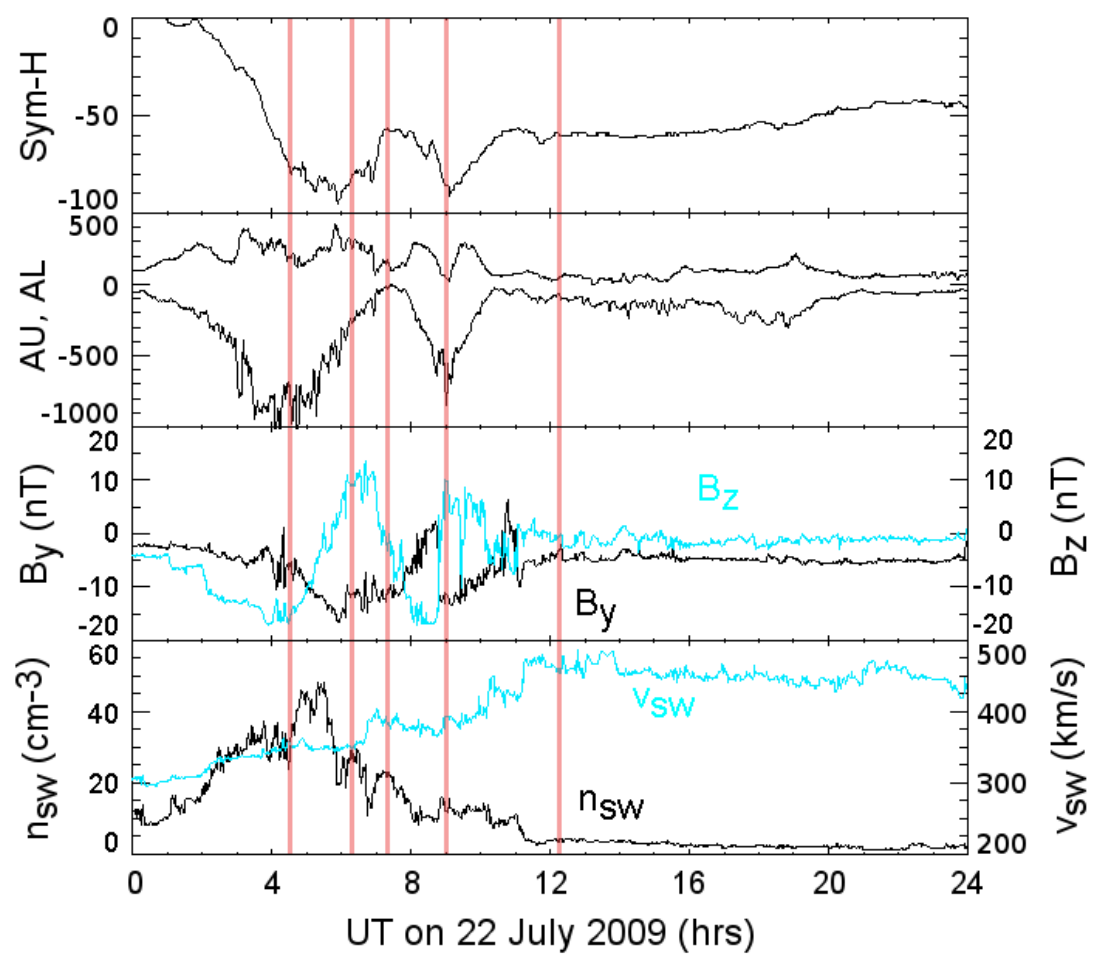

Figure 4.1: Geomagnetic indices and ACE data provided by OMNIWeb for the day of 22 July 2009. Red vertical bars indicate the times selected for analysis.

As is suggested by the AU and AL indices in Figure 4.1, observations of several substorms were recorded during this event. Table 4.1 shows the specific substorm times and groundbased observation locations, as provided through the SuperMAG substorm database. ${ }^{3,4}$ Four substorms are reported from 0249 UT to 0420 UT, which all have localized onset locations near $10 R_{E}$ and $\sim 5$ MLT in the magnetic equatorial plane. The T96 magnetic field model ${ }^{5}$ is used for the field line tracing, starting with the ground magnetic latitude and ground MLT provided by SuperMAG. The substorms observed at 0829 UT and 0854 UT map farther 
downtail and closer to midnight.

\begin{tabular}{ccc|cc}
\hline $\begin{array}{c}\text { Substorm timing and location } \\
\text { from SuperMAG }\end{array}$ & \multicolumn{2}{c}{$\begin{array}{c}\text { Equatorial location } \\
\text { from CRCM-T96 }\end{array}$} \\
\hline UT & Mag Lat [deg] & MLT [hrs] & $R_{e q}\left[R_{E}\right]$ & MLT $_{e q}[\mathrm{hrs}]$ \\
\hline 0249 & 66.48 & 4.62 & 10.5 & 4.5 \\
0317 & 66.48 & 5.07 & 10.8 & 4.7 \\
0347 & 67.40 & 5.95 & 10.9 & 5.5 \\
0420 & 66.48 & 6.10 & 10.5 & 5.6 \\
0829 & 66.33 & 1.74 & 24.0 & 1.1 \\
0854 & 66.33 & 2.16 & 22.5 & 1.6 \\
\hline
\end{tabular}

Table 4.1: Substorm parameters obtained through SuperMAG for 22 July 2009. The left 3 columns indicate the time at which the substorm was observed (left), the magnetic latitude of the ground observation (center) and the magnetic local time of the ground observation (right). The right 2 columns show the corresponding radius $R_{e q}$ (left) and $\mathrm{MLT}_{e q}$ (right) of the ground observation point mapped to the equatorial plane using the T96 field model.

The 22 July 2009 storm has been studied in many prior publications, including Fok et al., ${ }^{6}$ Valek et al., ${ }^{7,8}$ Ilie et al., ${ }^{9}$ Perez et al., ${ }^{10}$ and Ganushkina et al., ${ }^{11}$ and the reader is referred to these articles for informative discussions on the solar wind conditions and the magnetospheric response during this event. Significant portions of the forthcoming study of this event are taken from Elfritz et al. ${ }^{12}$ Fok et al. used CRCM to examine the impact of different magnetic field models (static and dynamic) on the inner magnetosphere during this event. ${ }^{6}$ Their simulations used the TM boundary condition model; the authors emphasize the importance of a dynamic field model for producing simulated TWINS ENA fluxes and THEMIS-D fluxes that favorably compare with observations. In addition, the ion temperatures discussed in this paper were used by Keesee et al. ${ }^{13}$ to study magnetospheric ion temperature evolution 
during the main phase of this storm, as discussed in Section 4.1.2. New simulations of this storm are presented here to provide validation against these prior results. Our data-model comparison with THEMIS-D is in direct agreement with their results, shown in Section 4.1.4. It should be noted that the TM plasma sheet model is statistically invalid for most of this storm, because one or more of the solar wind conditions fall outside of the statistical limits discussed in Section 3.2.2 (see Figure 4.1).

\subsubsection{Storm-time Ion Temperatures}

2-D ion temperature maps derived from TWINS ENA images are presented in Figures 4.24.7 and discussed in the context of the corresponding storm phases. In each figure, 2-D ion temperature maps generated from single-spacecraft observations are shown in the top row. These observations are then mirrored about the midnight-noon meridian for equatorial pixels which have no ion temperature defined; this is the first step in preparing boundary conditions for CRCM. Remaining 'dataless' pixels are then filled with the average temperature, as discussed in Section 2.1. In the top row, the spacecraft identifier (TWINS 1 or TWINS 2) is labeled in the upper-right corner of each panel. It is important to note that maps for every integration period (20 actuator sweeps) are not necessarily shown. In the bottom row, the location of the CRCM simulation outer boundary is plotted over the temperature map in black for each time. The CRCM boundary conditions are updated in 10 second time steps, and the temperature in each pixel is interpolated linearly between the temperatures given in successive maps. Such successive maps are presented here as Figures 4.2-4.7, and the timestamp for each map is denoted above each panel. The timestamp is the center of the integration interval, explained in more detail in Section 3.3.

Keesee et al. ${ }^{13}$ presented 2-D maps of equatorially-projected ion temperatures for the 

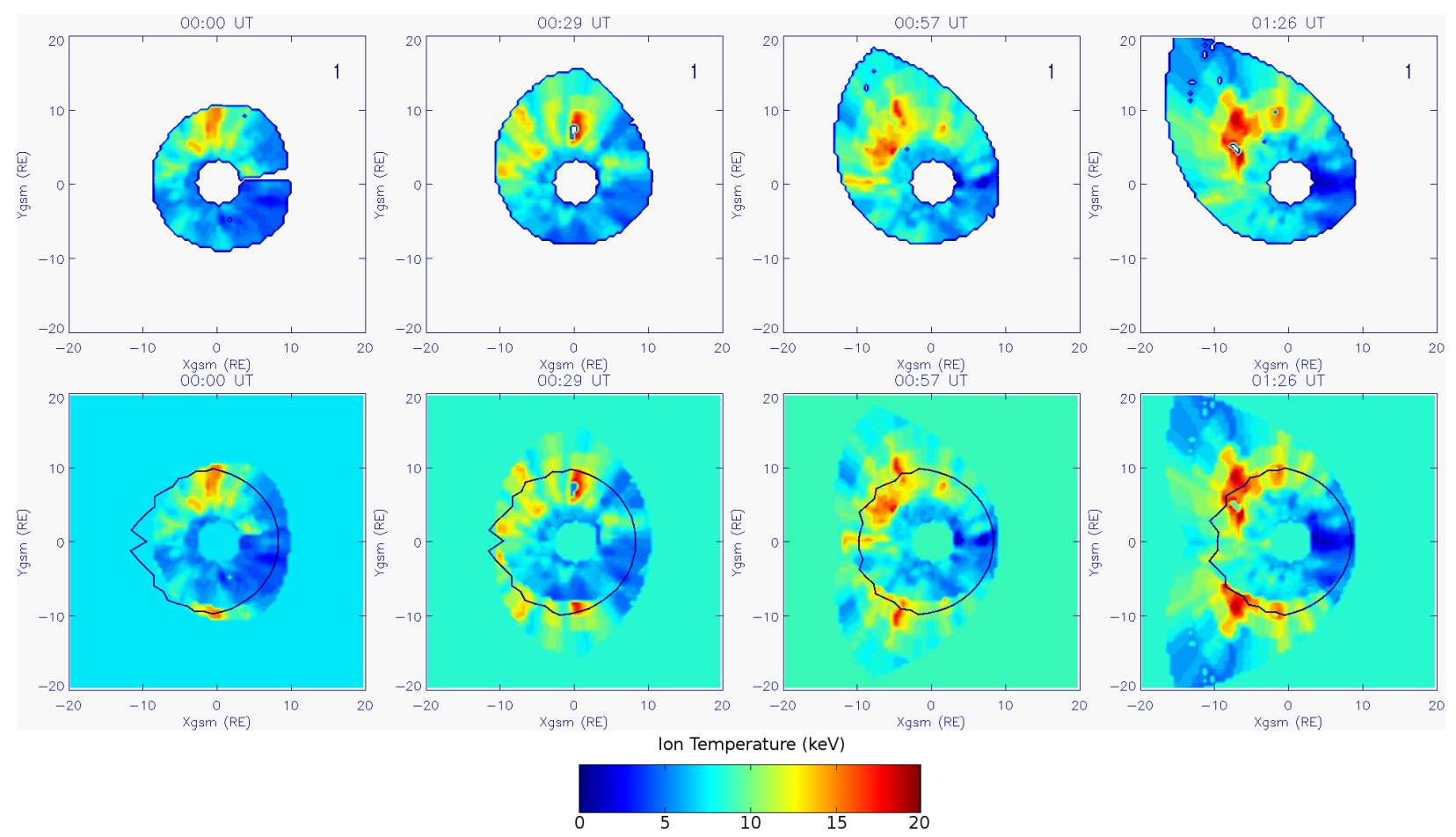

Figure 4.2: ENA-derived equatorial ion temperature maps for 0000-0126 UT on 22 Jul 2009. The top row shows the raw temperature calculations, and the bottom row shows the temperatures used for CRCM boundary conditions; good data are mirrored about the noon-midnight meridian and pixels with missing data are filled with averages. The time-varying CRCM outer boundary is overplotted in the bottom row.

interval 0000-0544 UT, corresponding to the main phase of this event. The authors reported observations of dawnward-directed convection of hot ions from the dusk sector, a feature which is characteristic of HSS-driven events. ${ }^{14,15}$ That time interval is decomposed into shorter intervals (faster cadence) between successive maps here, where maps are presented based on integration intervals of 20 actuator sweeps; the Keesee et. al. study presented temperature maps derived from 40 sweep intervals. Data corresponding to that time interval are shown in Figures 4.2-4.4.

Ion temperature maps generated from TWINS 1 ENA data are shown for 0000-0126 UT in Figure 4.2. During this time the dusk-side temperatures are considerably hotter than 

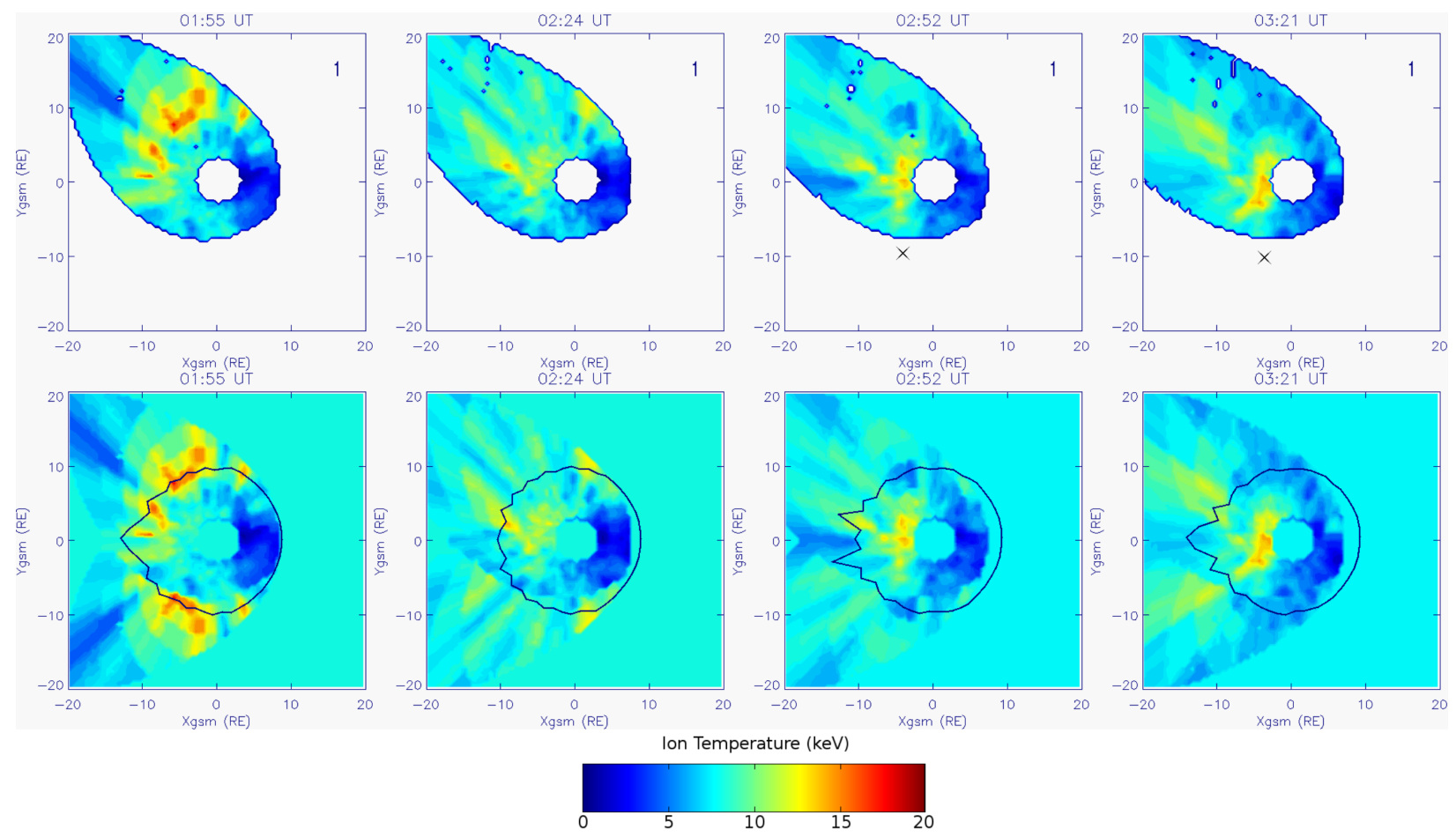

Figure 4.3: ENA-derived equatorial ion temperature maps for 0155-0321 UT on 22 Jul 2009. The top row shows the raw temperature calculations, and the bottom row shows the temperatures used for CRCM boundary conditions; good data are mirrored about the noon-midnight meridian and pixels with missing data are filled with averages. The time-varying CRCM outer boundary is overplotted in the bottom row.

those at dawn, although the TWINS 1 field of view limited coverage in the dawn sector. Near the end of this interval, the Sym-H index turned negative indicating the beginning of the main phase; this Sym-H decrease was induced by a strengthening southward IMF $B_{z}$ component. By $0200 \mathrm{UT}, B_{z}$ again decreases, to $\approx-12 \mathrm{nT}$, as the main phase continues. In Figure 4.3 we find that the hot dusk-side patch from Figure 4.2 convects dawnward across the midnight meridian as the peak temperature decreases. This decrease is due to ions of different energy following different drift paths, which spatially disperses hot, localized populations when strong convection fields are present in the inner magnetosphere. ${ }^{16,17}$ During this interval 0155-0321 UT, the IMF $B_{z}$ continues to decrease while the solar wind ram 

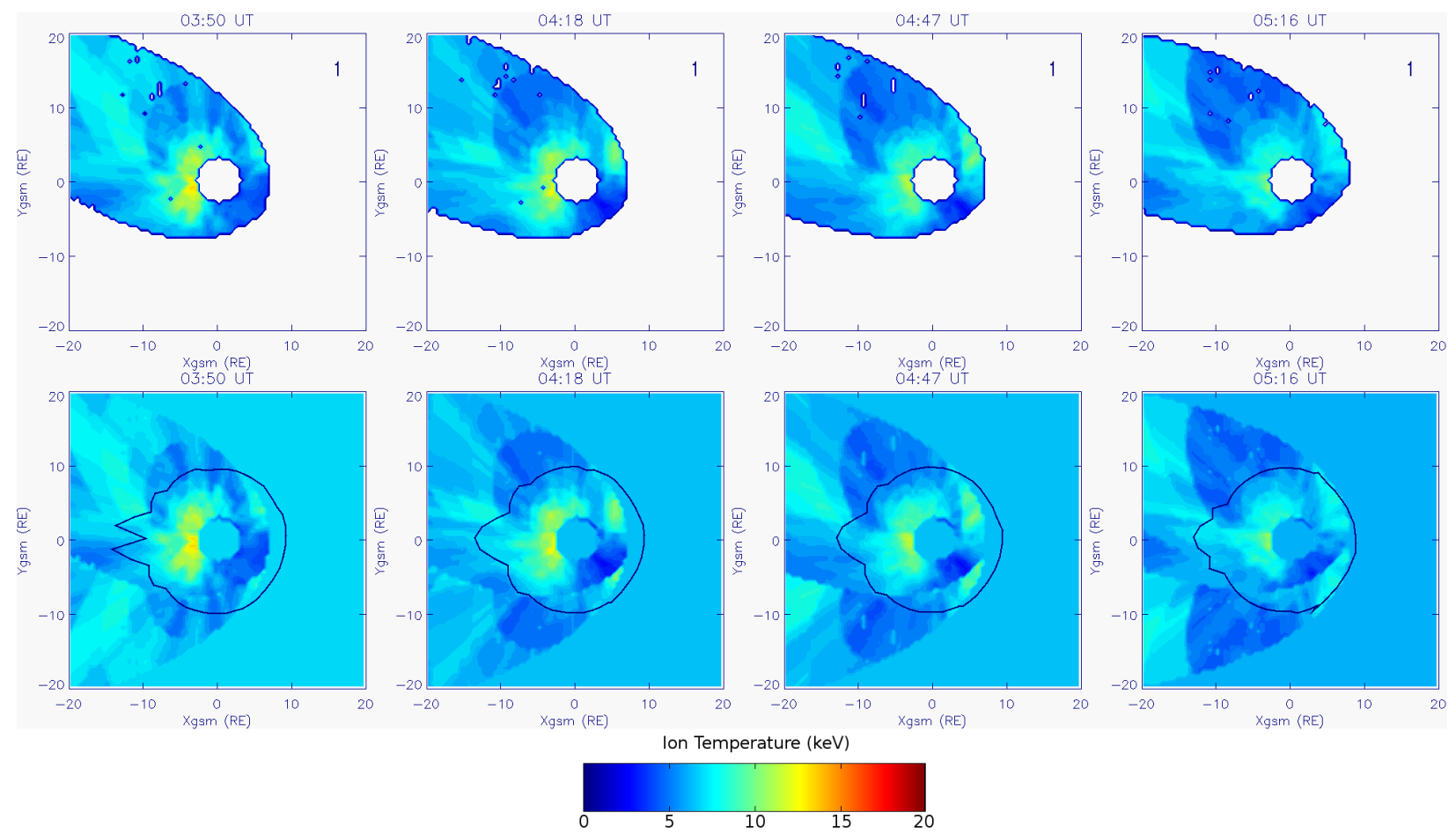

Figure 4.4: ENA-derived equatorial ion temperature maps for 0350-0516 UT on 22 Jul 2009. The top row shows the raw temperature calculations, and the bottom row shows the temperatures used for CRCM boundary conditions; good data are mirrored about the noon-midnight meridian and pixels with missing data are filled with averages. The time-varying CRCM outer boundary is overplotted in the bottom row.

pressure increases. The AL index drops below $500 \mathrm{nT}$, and semi-regular substorm activity is recorded, as is shown in Table 4.1. Any heating associated with the substorm at 0249 UT is not necessarily visible in the 0252 UT-centered temperature map in Figure 4.3. The black $\mathrm{X}$ in the top panel shows the location of the substorm onset mapped to the GSM equatorial plane. Similarly, the black X in the 0321 UT-centered panel shows the equatorial location of the substorm detected at 0317 UT. In both cases, the TWINS 1 field of view is not ideal for observations in that particular location over that time interval. Thus, localized heating or injection of hot ions associated with a substorm in those regions is not captured for use as boundary conditions. 

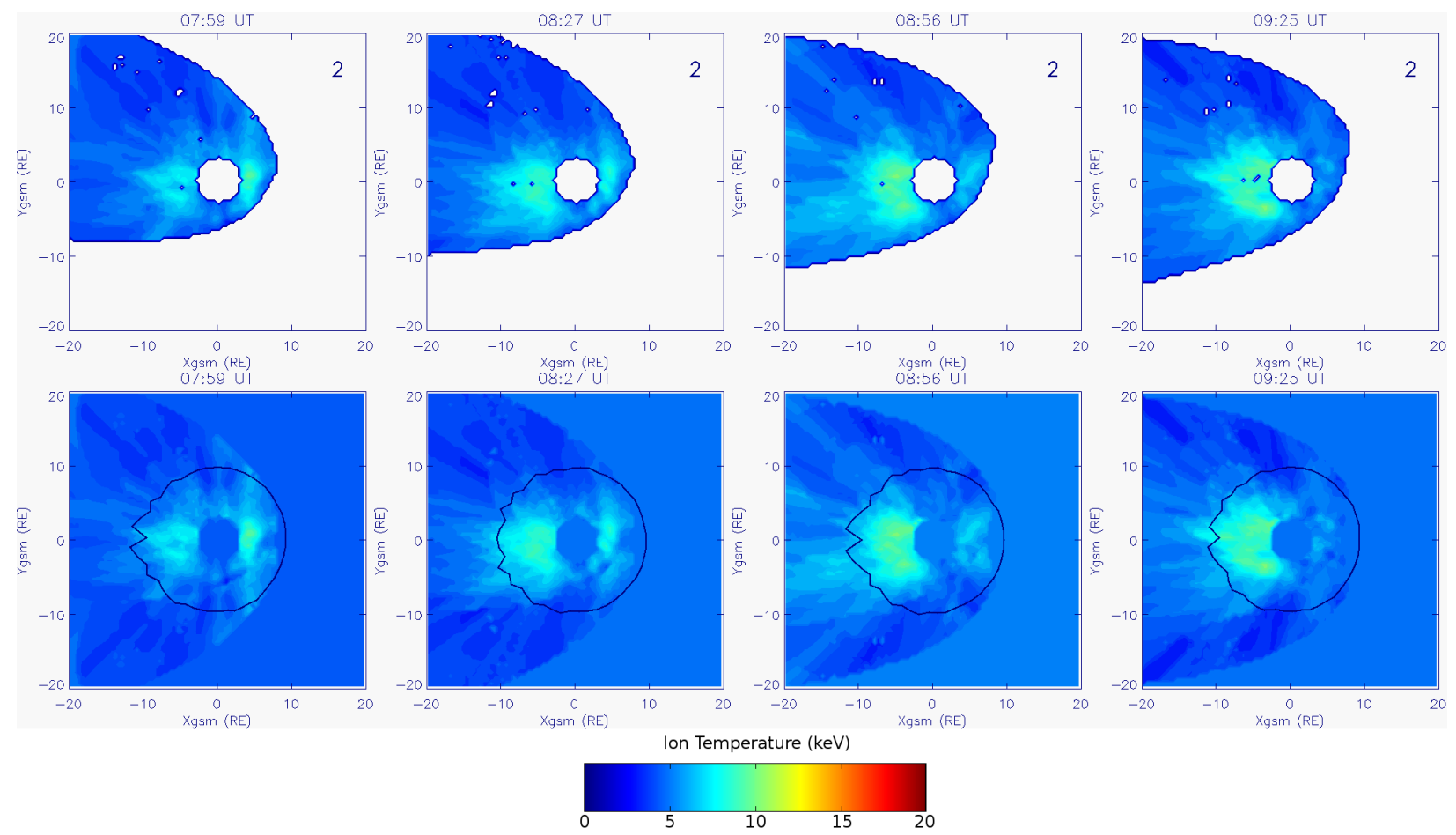

Figure 4.5: ENA-derived equatorial ion temperature maps for 0759-0925 UT on 22 Jul 2009. The top row shows the raw temperature calculations, and the bottom row shows the temperatures used for CRCM boundary conditions; good data are mirrored about the noon-midnight meridian and pixels with missing data are filled with averages. The time-varying CRCM outer boundary is overplotted in the bottom row.

Temperatures shown in Figure 4.4 correspond to the end of the main phase, ending with the northward IMF turning at 0530 UT. Over this interval the peak ion temperatures decrease, although a warm population inside of geosynchronous orbit is sustained. The 0420 UT substorm at 10.5 $R_{E}$ and 5.6 MLT does not appear to show up in the temperature map (at $0418 \mathrm{UT}$ ). This is because ions sourced at that equatorial location would immediately drift towards noon and be lost at the dayside magnetopause; see the drift paths discussed later in this chapter. Thus, the region through which the ions would drift is not within the TWINS 1 coverage area during this interval. In addition, TWINS 2 was too close to perigee to provide useful ENA images. As with the earlier substorms, any local substorm-related 
effects that could be found in the ion temperature map are not included in the boundary conditions because those regions were outside of the TWINS field of view.

Temperatures for the interval 0759-0925 UT are shown in Figure 4.5, during which time IMF $B_{z}$ was first strongly southward $(-15 \mathrm{nT})$ and turned strongly northward $(+9 \mathrm{nT})$ by 0900 UT. A substorm expansion phase is observed early in this interval (Figure 4.1), and substorms were detected through SuperMAG at 0829 UT and 0854 UT (see Table 4.1). The substorms both occurred near 1.5 MLT and $23.0 R_{E}$, which is just beyond the spatial extent of the temperature map. However, it may easily be identified that over this interval the peak inner magnetospheric ion temperatures are localized to the 0-2 MLT sector, and ion drift paths in that region map back to the inferred substorm onset location. Thus, the ENA-based temperature maps over this interval 0800-0930 UT may be capturing some ion heating due to the substorm activity observed during this time. The inner magnetospheric population at, for example, the region near geosynchronous midnight is heated from $\sim 7 \mathrm{keV}$ at $0759 \mathrm{UT}$ to $\sim 11 \mathrm{keV}$ at $0856 \mathrm{UT}$. This change in local temperature over the course of the substorm expansion phase is in good agreement with Baumjohann et al., ${ }^{18,19}$ wherein the authors explain why a 2-3 keV ion temperature increase can be expected during a substorm expansion phase. In the bottom row of Figure 4.5 we see that the heating is primarily confined to the region just inside of the simulation boundary, but the boundary does sample some of the nearby warmer population in the midnight-dawn quadrant. Observational signatures of the substorm activity during this interval were also picked up by THEMIS spacecraft, and will be discussed in Section 4.1.4 of this Chapter.

Ion temperature maps for the early recovery phase are provided in Figure 4.6, corresponding to the interval 1051-1436 UT. Global TWINS 2 temperatures remain very cool from roughly 1000-1230 UT, as illustrated at 1051 UT. Over this interval, the solar wind 

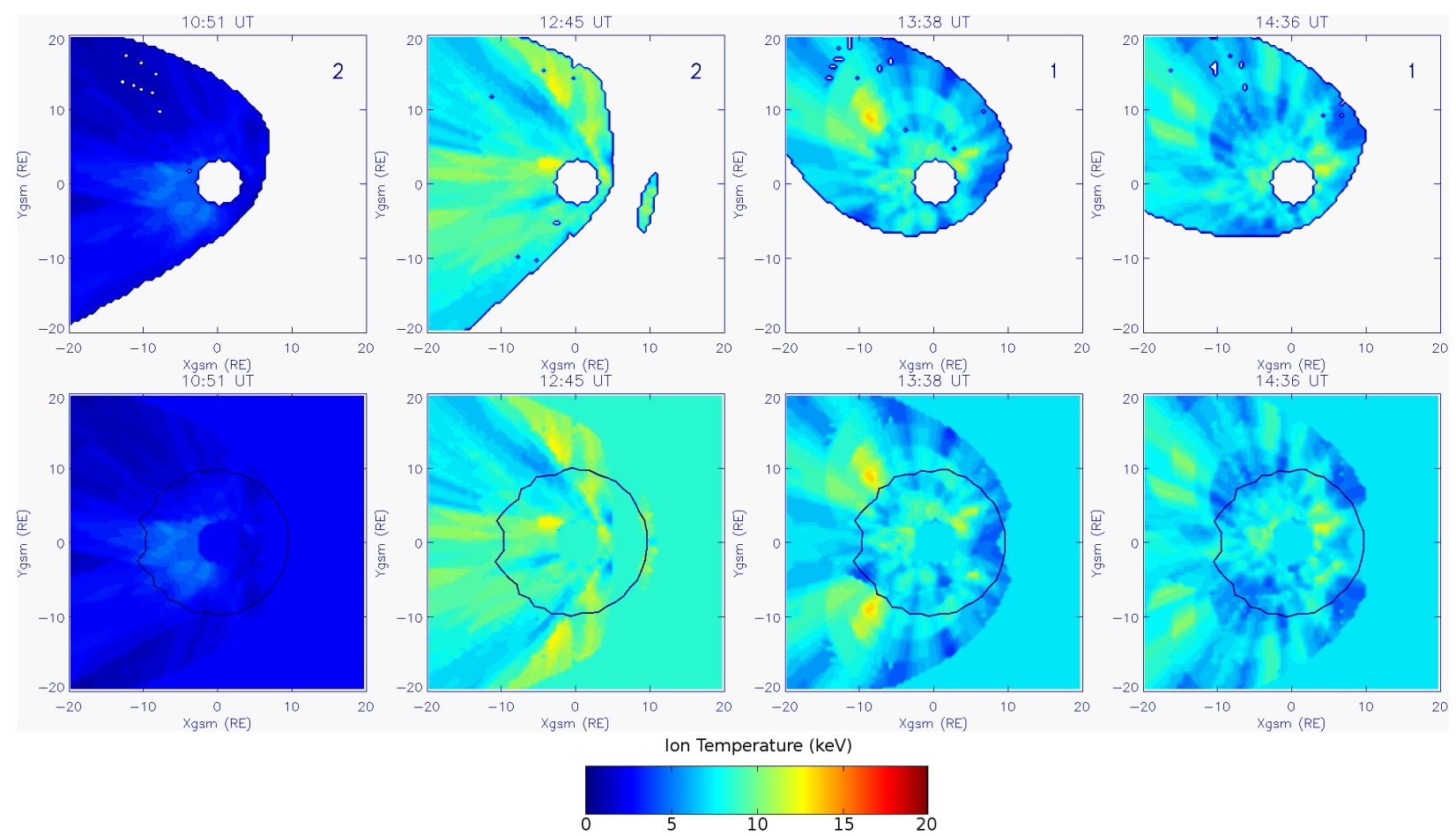

Figure 4.6: ENA-derived equatorial ion temperature maps for 1051-1436 UT on 22 Jul 2009. The top row shows the raw temperature calculations, and the bottom row shows the temperatures used for CRCM boundary conditions; good data are mirrored about the noon-midnight meridian and pixels with missing data are filled with averages. The time-varying CRCM outer boundary is overplotted in the bottom row.

ram pressure decreases by a factor of approximately three, allowing the magnetosphere to expand. During this process, stored magnetic energy is released and heats the existing magnetospheric ion population. This is evident by $1245 \mathrm{UT}$ and later into the recovery phase. The global temperatures remain elevated and roughly constant in time, as observed by TWINS 1 and 2, until about 1800 UT. After this time only TWINS 2 was in a near-apogee viewing location and TWINS 1 data is not available for the late recovery phase beyond about 1740 UT. Figure 4.7 shows the late recovery phase temperatures. A rather large, perhaps artificial, increase in peak global temperature is found in the final two panels, and the premidnight sector is found to be significantly cooler than the post-midnight temperatures. This 
'artificial' relative increase could be due to our comparing disparate spacecraft observations. As discussed in Section 2.3.1, the TWINS 1 and 2 ENA imagers are not cross-calibrated in real time, and thus it is not unusual to see differences in overall temperature magnitudes when comparing TWINS 1 with TWINS 2.
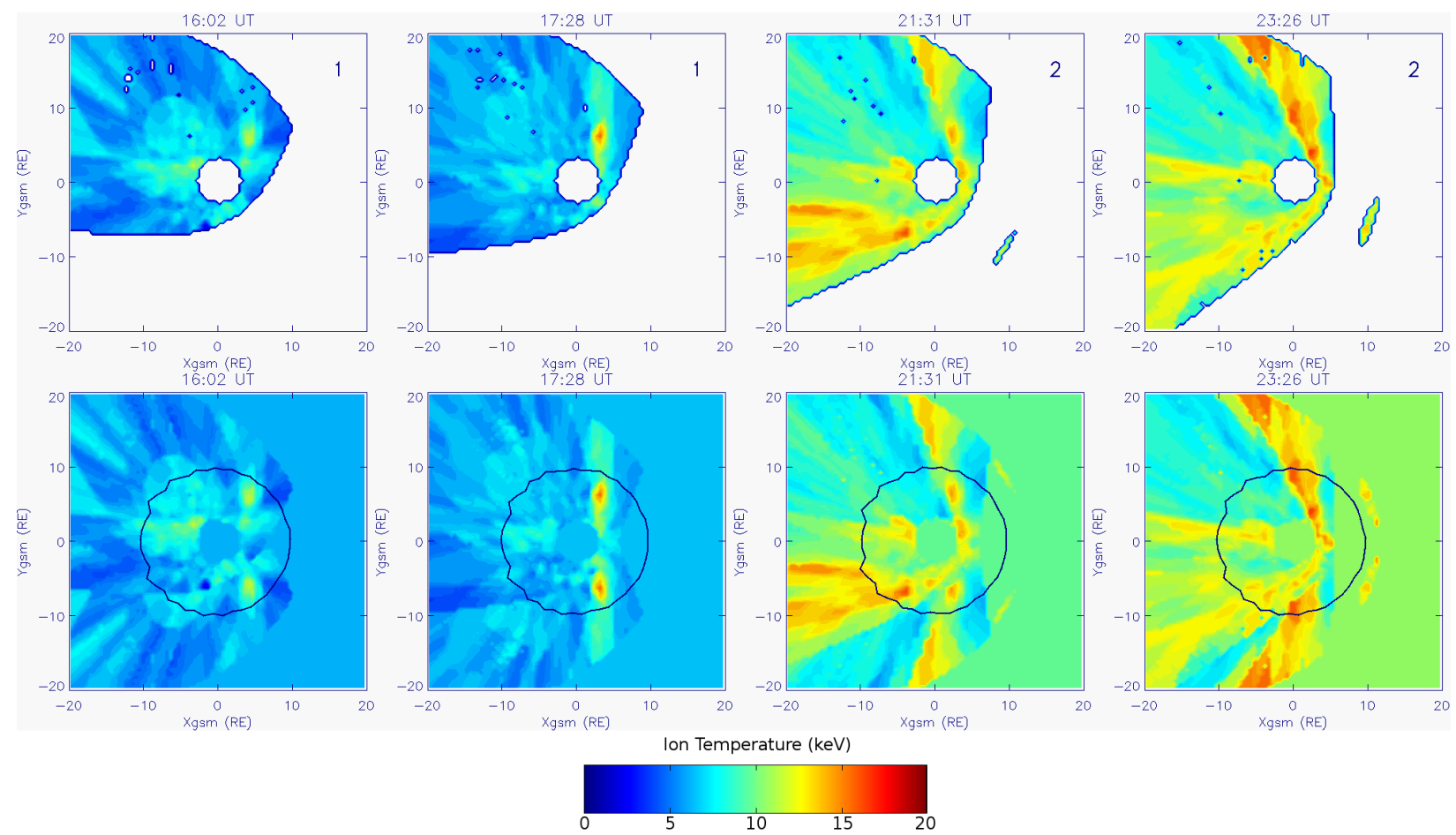

Figure 4.7: ENA-derived equatorial ion temperature maps for 1602-2326 UT on 22 Jul 2009. The top row shows the raw temperature calculations, and the bottom row shows the temperatures used for CRCM boundary conditions; good data are mirrored about the noon-midnight meridian and pixels with missing data are filled with averages. The time-varying CRCM outer boundary is overplotted in the bottom row.

Figure 4.8 shows a comparison between the ENA-derived ion temperature boundary conditions $T_{E N A}$ and the TM ion temperature boundary conditions $T_{T M}$, in the bottom and top panels respectively. Since the TM model is technically only valid beyond $10 R_{E}$, we assign $10 R_{E}$ ion temperatures when the CRCM outer boundary for that simulation is located inside of $10 R_{E}$. Note the fine spatial and temporal structure visible in the $T_{E N A}$ 
panel; these features are embedded in larger scale, slowly-varying temperature fluctuations. These larger scale variations appear to have similar characteristic timescales as $T_{T M}$, but very different magnitudes, and are also similar to solar wind timescales during this event (see Figure 4.1). One example of this fine spatial-temporal variation can be seen in the bottom panel of Figure 4.8 near midnight around 0800 UT. At that time and location the average boundary temperature is approximately $5 \mathrm{keV}$, but a superimposed dynamic warmer patch $(8 \mathrm{keV})$ is visible near midnight. Multiple substorms occurring during that time interval are likely responsible for this warm injection, as discussed with reference to Figure 4.5. We also see these fast, transient structures in the ENA-derived temperatures when comparing $T_{T M}$ and $T_{E N A}$ boundary conditions for other event simulations, including the 2010 Jun 4, 2011 May 28, and 2013 Oct 30-31 events. The larger-scale, slower features are still found to vary on similar timescales during those events, while showing different magnitudes and still remaining consistent with solar wind timescales. While we do not expect these fine transient structures to have a significant, long-term impact on the storm-time ring current, it is evident that the time-dependent energy distributions will certainly be affected. However a temporary, localized change in boundary temperature will in general only affect energy distributions on timescales comparable to drift timescales, which are typically hours to tens of hours for ion energies of a few $\mathrm{keV}$. The present analysis does not include timescales of this order, as we focus our investigation to key times which are separated by periods of hours. Thus, a more detailed analysis is required to comment on any effects associated with these transient features, and such an analysis is beyond the scope of this work.

Early in the initial southward $B_{z}$ phase, $T_{E N A}$ are considerably higher than $T_{T M}$, with a hot $(\sim 20 \mathrm{keV})$ patch in the pre-midnight sector. The enhanced dusk-side ion temperatures are consistent with the superposed epoch analysis of HSS-driven storms performed by Den- 

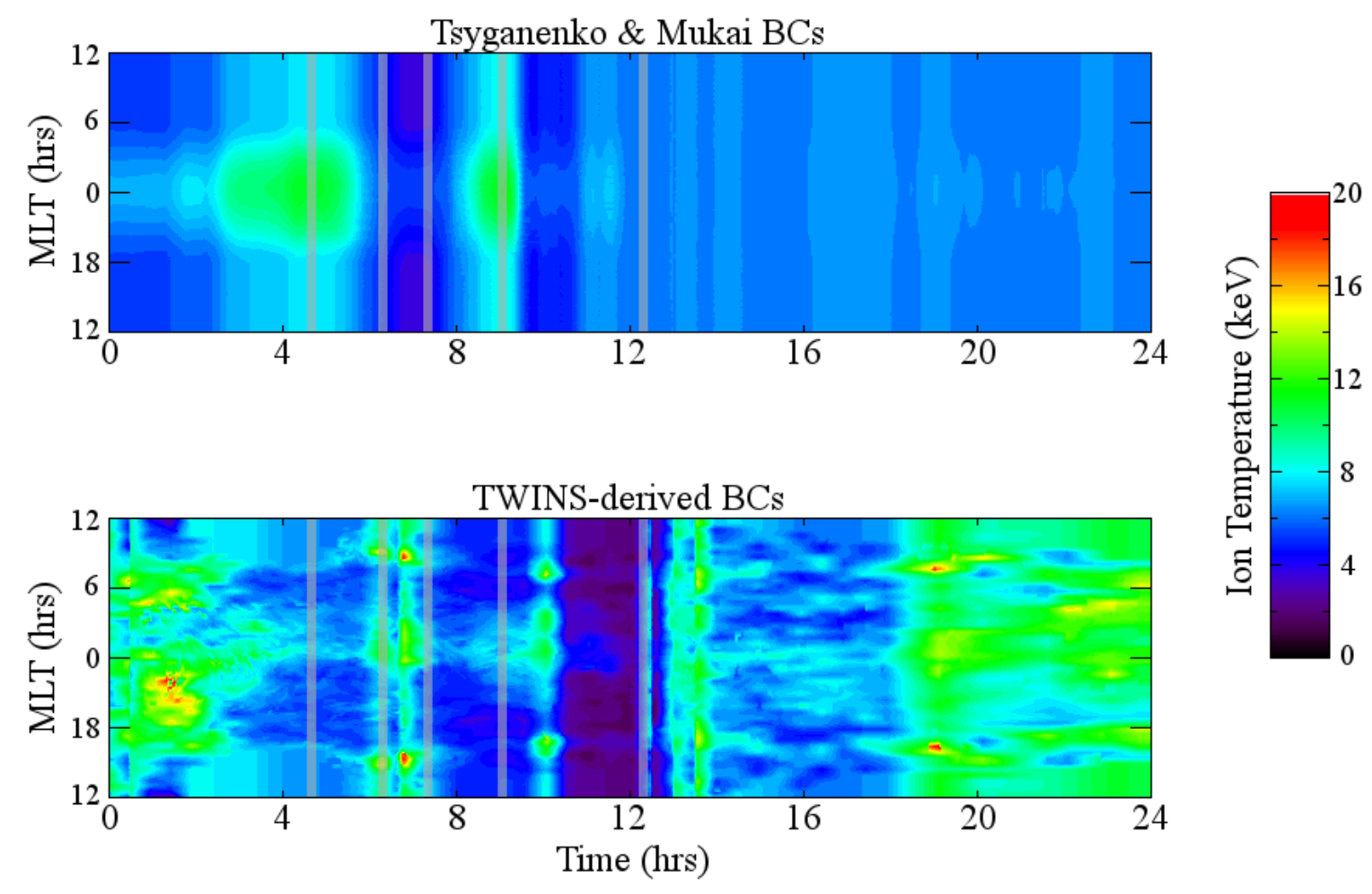

Figure 4.8: Ion temperature boundary conditions used in the TM (top) and ENA (bottom) simulations. ENA-derived temperatures (bottom) are interpolated to a 10second time step. Temperature is plotted on a $20 \mathrm{keV}$ colorbar versus time and MLT. Gray vertical bars indicate times selected for analysis, the same as in Figure 4.1

ton and Borovsky. ${ }^{14}$ Note that the TM model produces temperatures that are highest at, and symmetric about, midnight. Starting near $0200 \mathrm{UT}, T_{T M}$ increases globally and $T_{E N A}$ decreases globally while maintaining a weak dawn-dusk asymmetry until 0330 UT. During the northward phase after the Sym-H minimum at $0530 \mathrm{UT}$, hotter $T_{E N A}$ are briefly found at the simulation boundary, while the TM model predicts rather rapid cooling down to roughly $3 \mathrm{keV}$. Following the subsequent southward turning at $0715 \mathrm{UT}$, the distribution at the $T_{E N A}$ boundary abruptly cools, while the $T_{T M}$ boundary gradually heats again. During the initial short northward phase beginning at 0930 UT, hotter populations are found at dawn and dusk in the ENA-derived temperatures. A global decrease in $T_{E N A}$ follows, where they 
remain constant at $2 \mathrm{keV}$ for nearly 2 hours. The extended recovery phase follows, where $T_{E N A}$ show large-scale ion heating while still exhibiting small-scale structure. Another global increase in $T_{E N A}$ occurs at $1800 \mathrm{UT}$, which persists through the end of the simulation time frame.

In the following section, we present a comparison of the simulated $\mathrm{H}^{+}$pressure, and the energy- and time-dependence of the $\mathrm{H}^{+}$flux within the simulation domain. Our comparison highlights differences observed between simulations using the different outer boundary condition models previously discussed.

\subsubsection{Simulation Results}

All simulations discussed here begin at 2000 UT on 21 July 2009 with an empty magnetosphere, and run for 28 hours through 0000 UT on 23 July 2009. In this section, we focus on the 24 hour period of 22 July. Figure 4.9 shows a comparison of Sym-H* values for this period. The Sym-H* calculated from 1-minute OMNIWeb data is in black, and is the pressure-corrected Sym-H with contributions from magnetopause currents and currents induced within the Earth removed. The functional relation for converting observed Sym-H data (courtesy of OmniWeb) to Sym-H* is given by ${ }^{6,20}$

$$
\mathrm{Sym}-\mathrm{H}^{*}=\frac{\mathrm{Sym}-\mathrm{H}}{1.5}-15.8 \sqrt{P_{s w}}+20
$$

where $P_{s w}$ is the solar wind pressure in nanoPascals $(\mathrm{nPa})$. The solid red and blue lines correspond to Sym-H* values calculated by feeding the total simulation energy into the

Dessler-Parker-Sckopke (DPS) relation; ${ }^{21,22}$ the dashed red and blue lines show the contribution to Sym-H* from the total energy inside of geosynchronous orbit, also calculated using 
the DPS relation. The dotted red and blue lines show the same as the solid lines, except with losses due to the changing magnetic field also subtracted off. During the main phase and early recovery phase, higher boundary ion temperatures result in an increase in the total simulation energy and thus a corresponding decrease in the predicted Sym- $\mathrm{H}^{*}$. An example of this is found in the initial, brief recovery phase from 0600-0730 UT (see Figure 4.9). During this time, the $T_{E N A}$ boundary conditions are considerably hotter than those given by the TM model (see Figure 4.8), resulting in a pronounced Sym-H* minimum that better agrees with the observed Sym-H*; note that such a pronounced Sym- $\mathrm{H}^{*}$ minimum is not present in the simulation utilizing $T_{T M}$ boundary conditions.

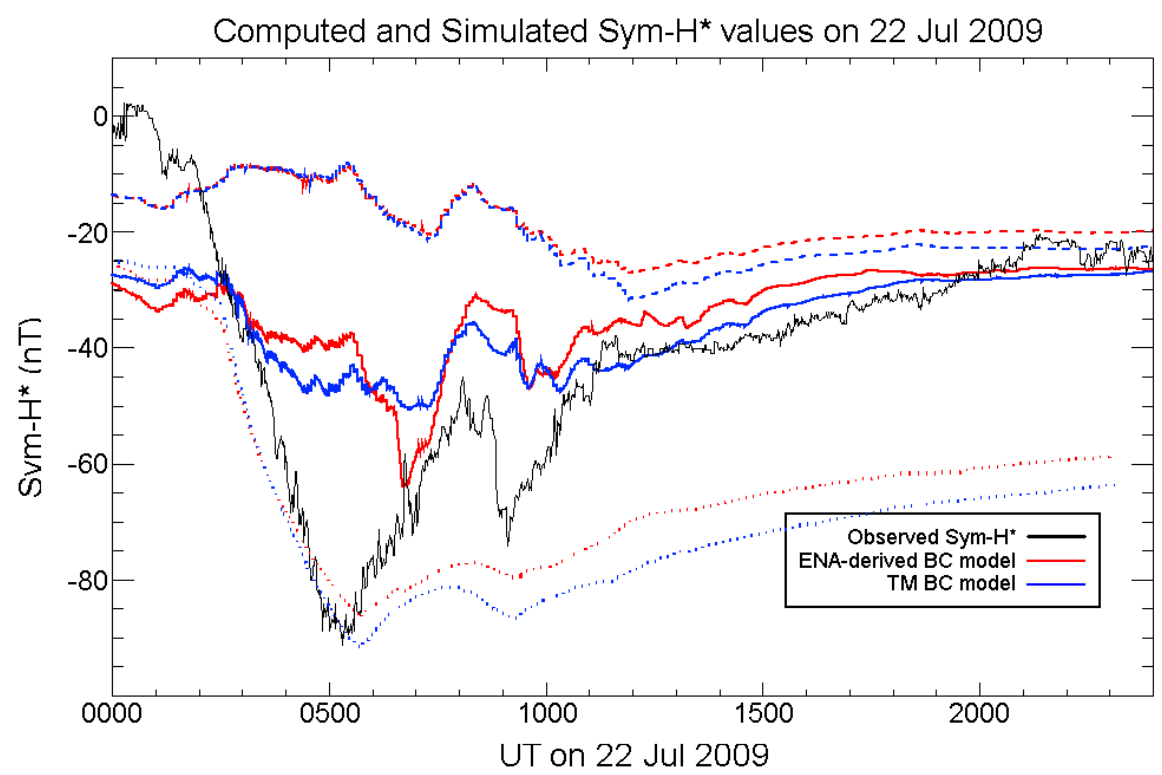

Figure 4.9: Comparison of Sym-H* values for the 22 Jul 2009 event. The black line shows the OmniWeb Sym-H values corrected by contributions of the solar wind pressure, magnetopause currents and currents induced within the Earth. The red and blue solid (dashed) lines show the total energy contained within the simulation domain (within geosynchronous orbit). The dotted lines show simulated Sym-H* with losses due to the changing magnetic field removed.

Figure 4.10 shows $\mathrm{H}^{+}$drift paths for a southward $B_{z}$ interval (0430 UT, left column) 


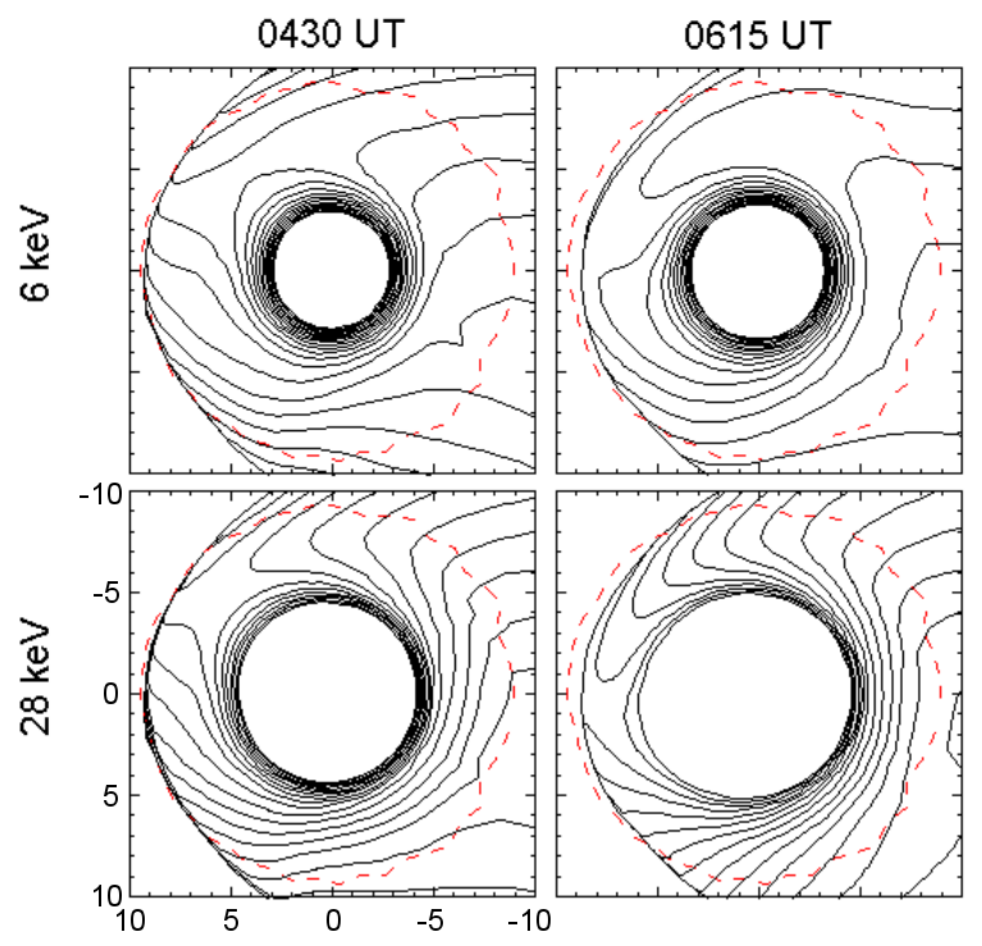

Figure 4.10: Comparison of drift paths for $6 \mathrm{keV}$ (top row) and $28 \mathrm{keV}$ (bottom row) protons during an interval of strong southward IMF (0430 UT, left column) and a strong northward interval (0615 UT, right column). The red dashed line indicates the location of the simulation's dynamic outer boundary at each time. Each panel shows the $\mathrm{x}-\mathrm{y}$ plane in GSM coordinates.

and a northward $B_{z}$ interval (0615 UT, right column) to provide context for the following discussion, where the spatial distribution of simulated fluxes is interpreted in terms of fundamental plasma drifts. Since a drifting particle changes its kinetic energy along its drift path due to conservation of the first adiabatic invariant, one must specify the particle energy at a specific location to compute the drift paths. In the top row, drift paths are presented for 6 $\mathrm{keV}$ protons referenced at geosynchronous midnight. In the bottom row, the same location is chosen but for $28 \mathrm{keV}$ protons. The important feature that distinguishes low-energy and high-energy particle drifts is that higher energy particles experience a westward (duskward) gradient-curvature drift that dominates over the effect of the Earthward and dawnward $\vec{E} \times \vec{B}$ 
drift. The red dashed lines superimposed on the plots indicate the dynamic outer boundary of the simulation at the chosen times.

Figure 4.11 shows a comparison of simulated $\mathrm{H}^{+}$pressure $P$ with units of $\mathrm{nPa}$. Pressure is computed to include relativistic effects, and is calculated from pitch-angle averaged flux $J_{\alpha}$ with Equation 2.20. In these calculations, we take $E_{1} \approx 0 \mathrm{keV}$ and $E_{2}=250 \mathrm{keV}$, the full range of energy available from the CRCM data. The TM run is in the top row and the ENA run is in the bottom row, and the times shown for each column are the same as those indicated by vertical lines in Figures 4.1 and 4.8. The sun is to the left, and the white traces indicate geosynchronous orbit at $6.6 R_{E}$. The two boundary condition models produce $\mathrm{H}^{+}$ pressures that are very similar in their spatial configuration and in time, although some differences can be observed. Specifically the peak pressure from each boundary condition model occurs at nearly identical $L$-shells for all times shown, but the MLT dependence at 0715 UT and 0900 UT varies between boundary condition models. At 0715 UT the peak pressure occurs near $L=4.8$, and the $T_{T M}\left(T_{E N A}\right)$ run shows its region of highest pressure skewed towards dusk (dawn). This comparative shift in MLT could be explained by a hotter (cooler) population convecting in from the inner plasma sheet edge in the $T_{T M}\left(T_{E N A}\right)$ run, which is opposite from the actual boundary conditions shown in Figure 4.1, but convection is weak during the interval 0530-0715 UT and thus the boundary conditions have only a minor impact on the ring current during this period. As will be shown in the discussion of Figures 4.12 and 4.13 , the proton pressure in the $T_{T M}\left(T_{E N A}\right)$ run at $0715 \mathrm{UT}$ is dominated by the contribution of higher (lower) energy flux, resulting in ion drifts that are consistent with the relative shifts in pressures shown in Figure 4.11c. At 0900 UT and 1210 UT the peak pressure occurs near $L=4.3$; the difference at $0900 \mathrm{UT}$ is more subtle, but the $T_{E N A}$ run shows a slight duskward enhancement, while the $T_{T M}$ shows a more dawn-dusk symmetric 
pressure. At 1100 UT the boundary condition models result in very similar pressures on the dawn side, but the $T_{T M}$ run shows a slight enhancement in the noon-dusk quadrant, indicating more complete dayside ring current closure at that time.

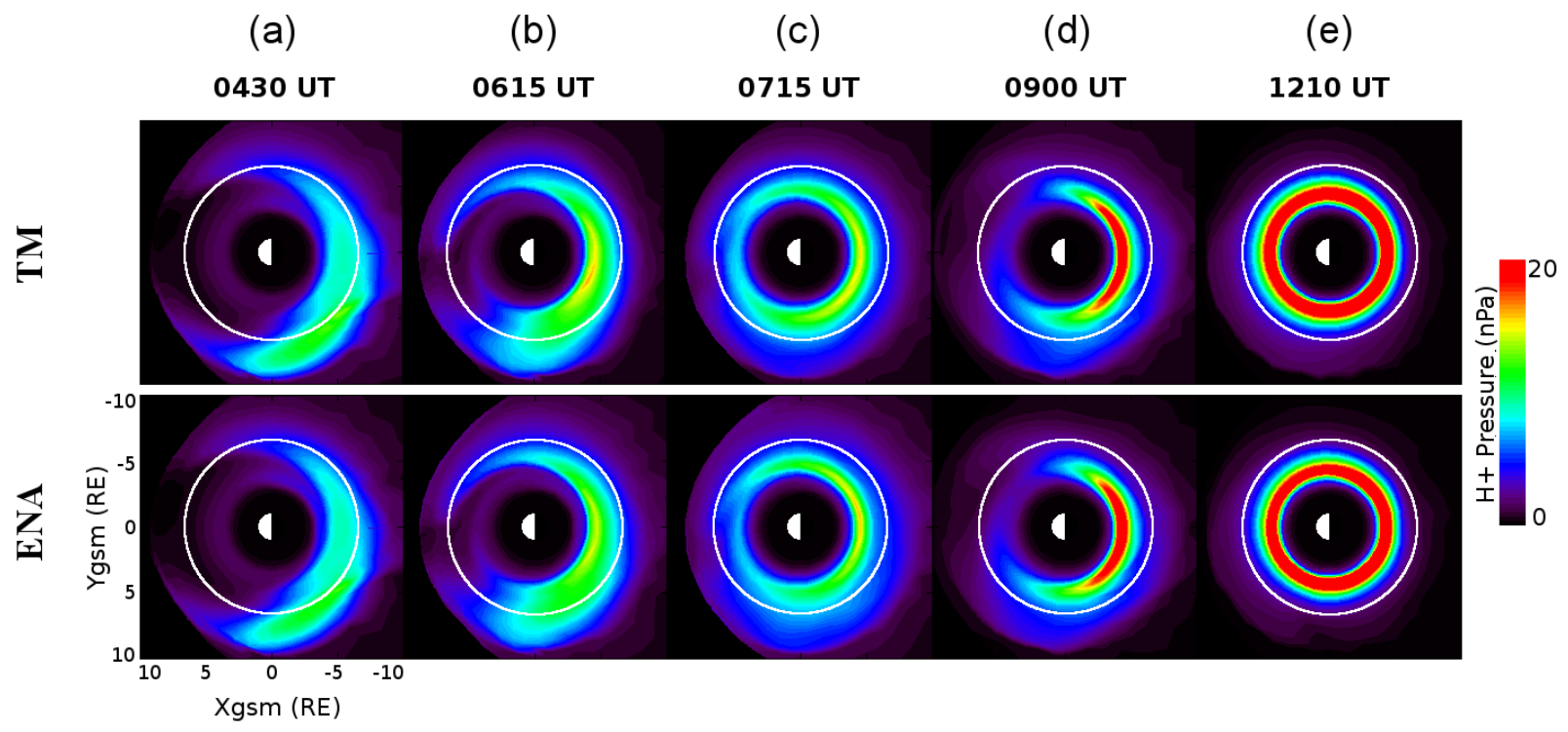

Figure 4.11: $\mathrm{H}^{+}$pressure $(\mathrm{nPa})$ using ion temperature boundary conditions from the TM model (top row), and ENA-derived model (bottom row) at five time steps (columns). Spatial coordinates in GSM are shown at the bottom left panel. In each panel, the sun is to the left and the white traces indicate geosynchronous orbit.

Figure 4.12 shows a comparison of simulated proton fluxes $\left(1 / \mathrm{keV} \mathrm{cm}^{2} \mathrm{sr} s\right)$ in the $6-18$ keV energy range (hereafter referred to as $12 \mathrm{keV}$ fluxes), and Figure 4.13 shows the fluxes for the 18-39 keV energy range (28 keV fluxes). During the initial southward $B_{z}$ interval from 0430-0530 UT, the midnight temperatures are $T_{T M} \approx 12 \mathrm{keV}$ and $T_{E N A} \approx 7 \mathrm{keV}$. As plasma is convected in during this period, the colder, largely low-energy population in the $T_{E N A}$ run is expected to $\vec{E} \times \vec{B}$ drift primarily dawnward. This is found to be the case in Figure 4.12a; the $T_{E N A}$ run produces larger $12 \mathrm{keV}$ fluxes than the $T_{T M}$ run, most noticeably in the midnight-dawn quadrant. In addition, the $12 \mathrm{keV}$ flux from the $T_{E N A}$ run has a localized peak (around $L=5-6.6$ ) while the flux provided by the $T_{T M}$ boundary conditions shows a 
much more diffuse distribution in $L$-shell. The $28 \mathrm{keV}$ fluxes however agree in magnitude and in spatial distribution during this time period, as shown in Figure 4.13a.

When the IMF turns northward near 0530 UT, inner magnetospheric drift paths change from open to closed, resulting in higher dayside fluxes until the subsequent southward turning at $0715 \mathrm{UT}$. At $0715 \mathrm{UT}$ (see Figure 4.12c), the $T_{E N A}$ run produces $12 \mathrm{keV}$ fluxes that are larger than those in the $T_{T M}$ run in both the post-dawn sector and at dusk. In addition, the $12 \mathrm{keV} \mathrm{H}^{+}$flux at midnight is more diffuse in the $T_{T M}$ run, and the flux peaks much closer to geosynchronous orbit than in the $T_{E N A}$ run. This stronger nightside penetration found in the $T_{E N A}$ run, despite the hotter boundary conditions, is due in part to the negligible convection associated with northward IMF. Because convection is effectively turned off during the northward interval from 0530-0715 UT, there are negligible Earthward fluxes from the plasma sheet during this time and thus the boundary conditions have a minimal impact on the energy dependence of inner magnetospheric fluxes. The colder plasma found at the $T_{E N A}$ simulation boundary during the earlier interval of southward IMF (ending at 0530 UT) penetrates to lower $L$-shells than in the $T_{T M}$ run and subsequently moves along closed drift paths after the northward turning at 0530 UT. Thus, it is not simply the temperature boundary conditions that determine how fluxes are distributed in energy during the event, but also the corresponding convection field strength. The $T_{T M}$ run shows slightly higher $28 \mathrm{keV}$ fluxes near dusk compared to the $T_{E N A}$ run (Figure $4.13 \mathrm{c}$ ), but the two boundary conditions produce otherwise spatially similar flux in the $28 \mathrm{keV}$ energy range.

The IMF turns strongly southward at $0800 \mathrm{UT}$, resulting in enhanced convection until another northward turning at 0845 UT. During this southward period the $T_{E N A}$ boundary conditions are cooler than those used in the $T_{T M}$ run. At 0900 UT the $T_{T M}$ run produces an enhancement of the $12 \mathrm{keV}$ flux in the pre-midnight sector (Figure 4.12d), consistent with a 


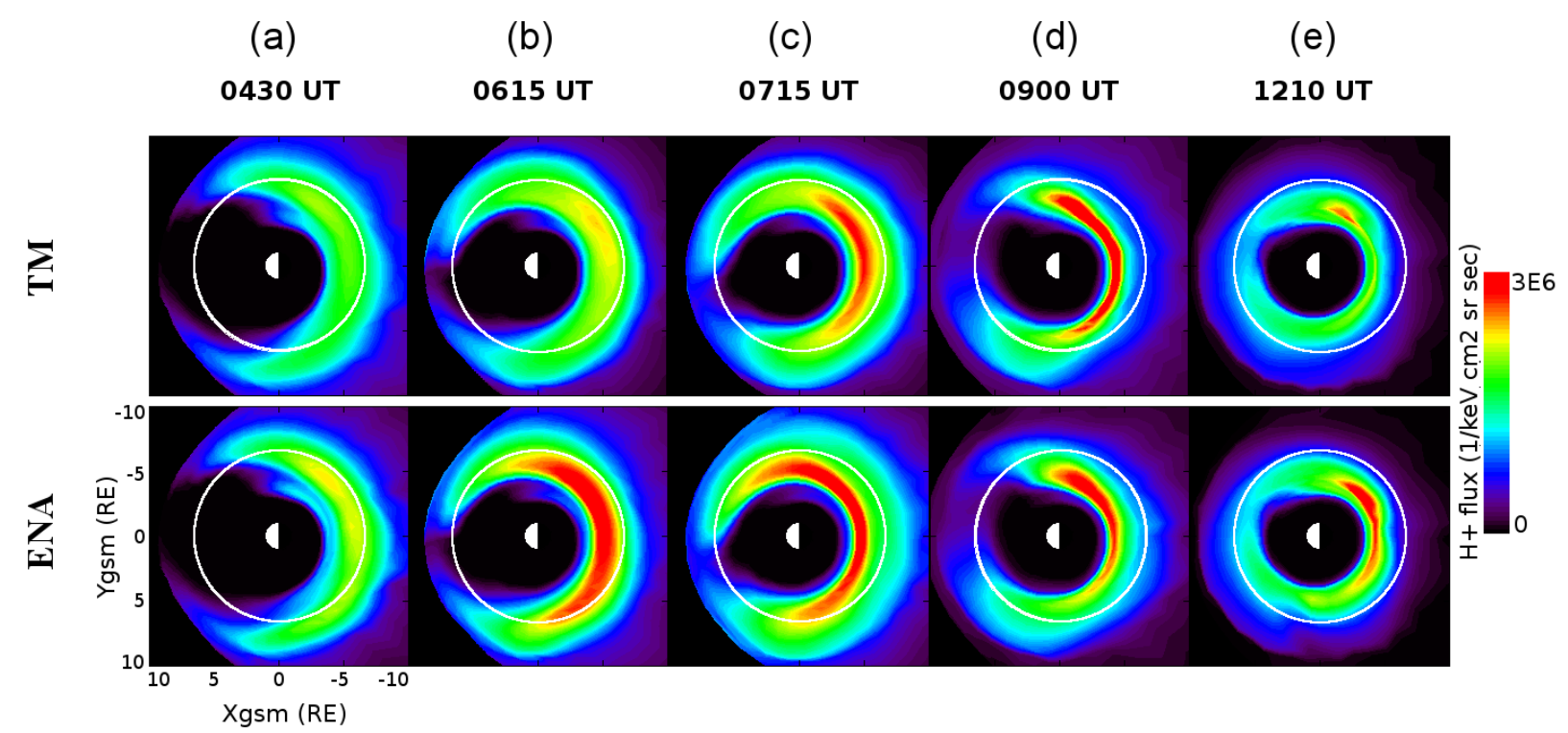

Figure 4.12: Simulated $6-18 \mathrm{keV}(12 \mathrm{keV}) \mathrm{H}^{+}$flux $\left(1 / \mathrm{keV} \mathrm{cm}^{2} \mathrm{sr} \mathrm{sec}\right)$ using ion temperature boundary conditions from the TM model (top row), and ENA-derived model (bottom row) at five time steps (columns). Spatial coordinates in GSM are shown at the bottom left.

higher-energy population experiencing westward gradient-curvature drift. The $28 \mathrm{keV}$ fluxes are similar in spatial structure and in magnitude (Figure 4.13d), although the region of peak pressure in the $T_{E N A}$ run covers a slightly larger extent in $L$-shell. At $0900 \mathrm{UT}$, the Sym-H reaches its second minimum, marking the beginning of the long recovery phase.

The IMF $B_{z}$ turns weakly southward for one hour beginning at $1000 \mathrm{UT}$, by which time clear high-speed stream signatures were observed by ACE (see Figure 4.1). At 1100 UT, the IMF $B_{z}$ abruptly relaxes to zero and the solar wind conditions become effectively steadystate. At this time, $T_{E N A} \approx 3-4 \mathrm{keV}$ and $T_{T M} \approx 6-8 \mathrm{keV}$, and the ring current begins to close as transport to the dayside is increased. At $1210 \mathrm{UT}$, there is an abrupt increase in nightside $T_{E N A}$ from $3 \mathrm{keV}$ to $8 \mathrm{keV}$. The $12 \mathrm{keV}$ ion fluxes in Figure 4.12e are consistent with these preceding boundary conditions; the $T_{E N A}$ run produces a strong gradient-curvature drift enhancement in the dusk-midnight sector out to $L=7$ due to the larger relative fraction of 


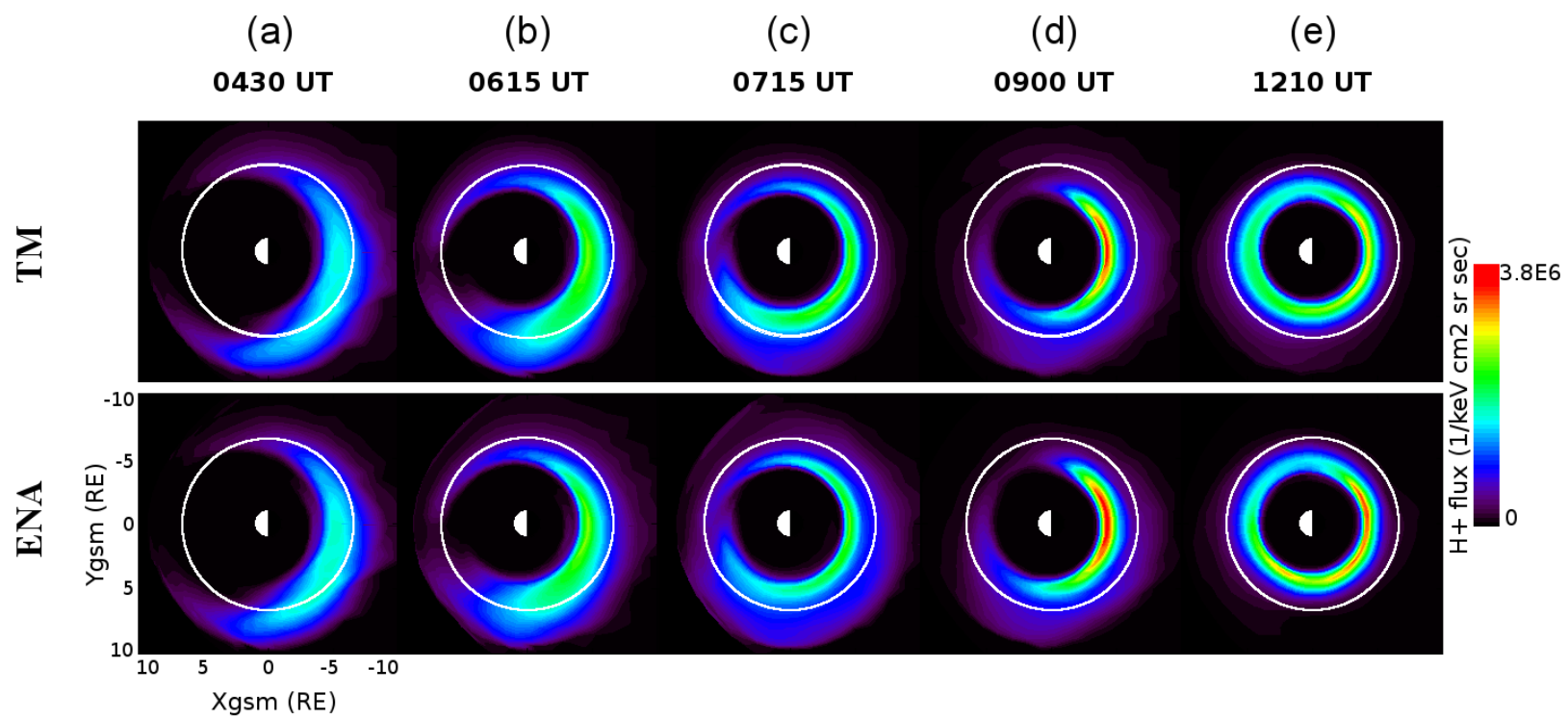

Figure 4.13: Simulated $18-39 \mathrm{keV}(28 \mathrm{keV}) \mathrm{H}^{+}$flux $\left(1 / \mathrm{keV} \mathrm{cm}^{2} \mathrm{sr} \mathrm{sec}\right)$ using ion temperature boundary conditions from the TM model (top row), and ENA-derived model (bottom row) at five time steps (columns). Spatial coordinates in GSM are shown at the bottom left.

high-energy protons, while the colder population convected in for the $T_{T M}$ run primarily experiences the $\vec{E} \times \vec{B}$ drift and convects to the dawn side. Higher $28 \mathrm{keV}$ fluxes at dusk and midnight are also produced in the $T_{E N A}$ run at $1210 \mathrm{UT}$, again consistent with a hotter boundary population.

To provide context for the comparison with observations in the next section, we present simulated differential fluxes at geosynchronous orbit in Figure 4.14. Each panel (a-e) of Figure 4.14 shows how the fluxes at geosynchronous orbit are distributed in energy and magnetic local time for each boundary condition model. At 0430-0615 UT the peak $T_{E N A}$ fluxes are larger in magnitude than those from the $T_{T M}$ run, although the distributions in MLT are similar. The $T_{E N A}$ run produces a $3 \mathrm{keV}$ enhancement near 20 MLT which is not found in the $T_{T M}$ run, indicating a localized low-energy population in the dusk-midnight quadrant. This feature is visible at both $0430 \mathrm{UT}$ and $0615 \mathrm{UT}$ (see Figure 4.14a, 4.14b). At 
0715 UT (Figure 4.14c), multiple differences may be observed. First, the TM run produces much higher $12 \mathrm{keV}$ fluxes at midnight than the ENA run; this is due to the more diffuse flux produced by the hotter TM boundary conditions prior to the northward turning near 0530 UT, which is consistent with the discussion of Figure 4.13c. In addition the ENA run shows a significant depletion of low-energy, 3-4 keV flux at midnight as well. In the dawn-noon quadrant, the TM run produces a depletion of low-energy flux. At 0900 UT (Figure 4.14d), both boundary condition models produce peak fluxes at 6 and 18 MLT (dawn and dusk), although the $T_{T M}$ run shows slightly larger absolute magnitudes. The $T_{T M}$ run also produces a low-energy $(1 \mathrm{keV})$ enhancement at dawn and fluxes near midnight that are considerably lower than those found in the $T_{E N A}$ run. Later in the recovery phase at $1210 \mathrm{UT}$ (Figure 4.14e), the fluxes calculated for each of the two boundary conditions models are similar in magnitude $(\approx 1.3 \mathrm{E} 6)$ but the energy dependence of flux at any given MLT is quite different; in particular, 1-10 keV fluxes in the pre-midnight sector are significantly higher in the $T_{E N A}$ run although both simulations produce a drop-out centered at midnight.

\subsubsection{Data-Model Comparisons}

In this section we take pitch-angle averaged fluxes obtained from CRCM and perform comparisons with two distinct observational data sets. Figure 4.15 shows a data-model comparison of ion temperatures at geosynchronous orbit for all MLT at each of the 5 times previously discussed. Here the temperatures calculated from CRCM fluxes (red and blue curves) are actually the "kinetic temperatures" $k_{B} T$ which may be calculated for any arbitrary distribution function (see e.g., Baumjohann and Treumann ${ }^{23}$ ) with the familiar $k_{B} T(\vec{r})=P(\vec{r}) / n(\vec{r})$, where $P$ is the trace of the pitch-angle averaged pressure tensor (which are presented in Figure 4.11; see equation 2.20) and $n$ is the local density, given by Equation 2.14. 


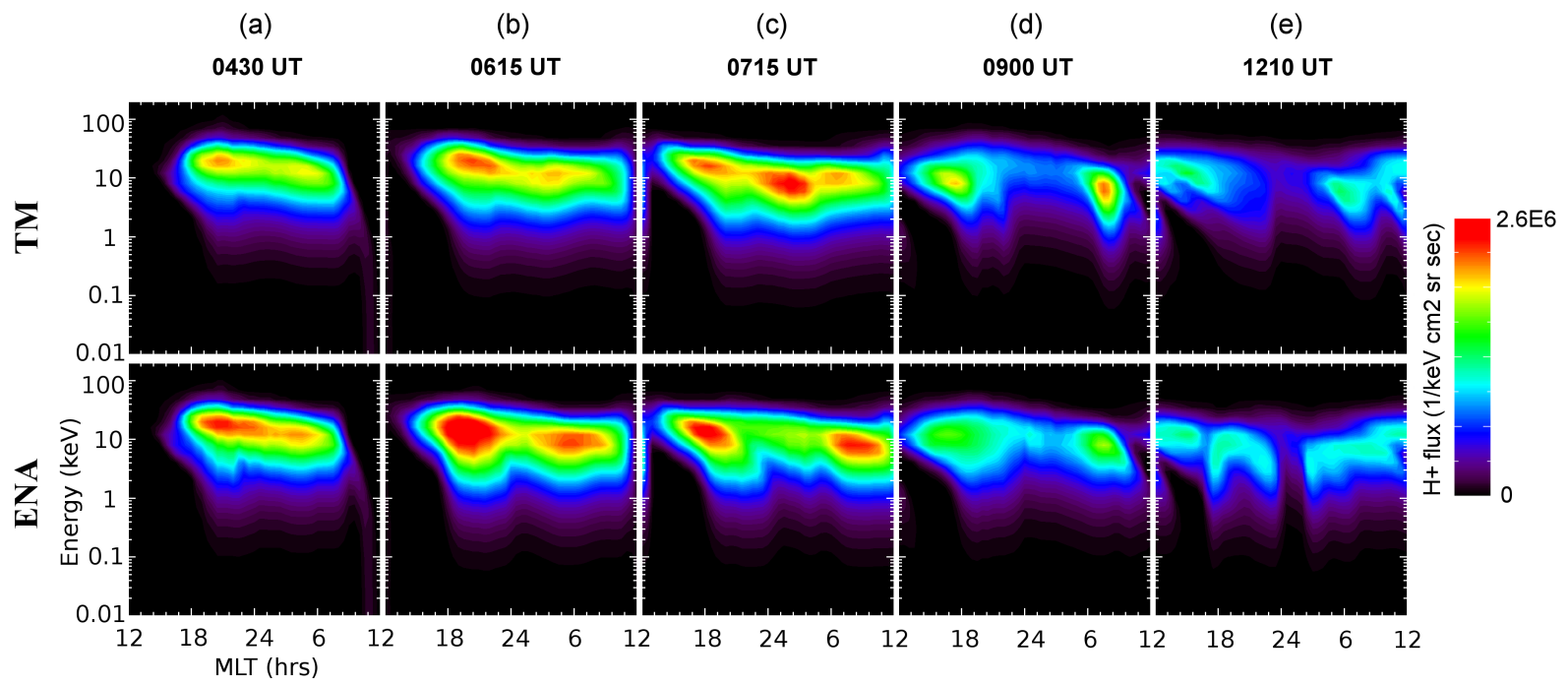

Figure 4.14: Simulated $\mathrm{H}^{+}$fluxes interpolated to geosynchronous orbit, as a function of energy and MLT, using the TM (top row) and ENA-derived (bottom row) boundary condition model. The colorbar is on a linear scale.

The temperature integrations based on CRCM fluxes are taken in the 0-25 keV energy range so as to be consistent with the THEMIS temperature moment integrations. These simulated geosynchronous temperatures are compared with geosynchronous observations extracted from TWINS ENA maps, which are shown in black. The TWINS-based temperatures are computed by integrating over the $0-32 \mathrm{keV}$ energy range. Keesee et al. ${ }^{13}$ discussed convection of hot ions during the main phase of this event, but only Figure 4.15a overlaps with that study. Zhang et al. ${ }^{24}$ showed clear dusk-dawn temperature asymmetries at geosynchronous orbit during moderate storms at solar minimum. They use MPA data to conduct a superposed epoch analysis and find that near zero epoch the observed dusk (dawn) temperatures are 9-10 keV (4-5 keV). Here we illustrate how well the CRCM simulations and TWINS observations agree with their results.

For all times shown (Figures 4.15a-e) both boundary condition models produce overall hotter (cooler) populations at dusk (dawn), which is consistent with our expectations from 
(a)

(b)

(c)

(d)

(e)

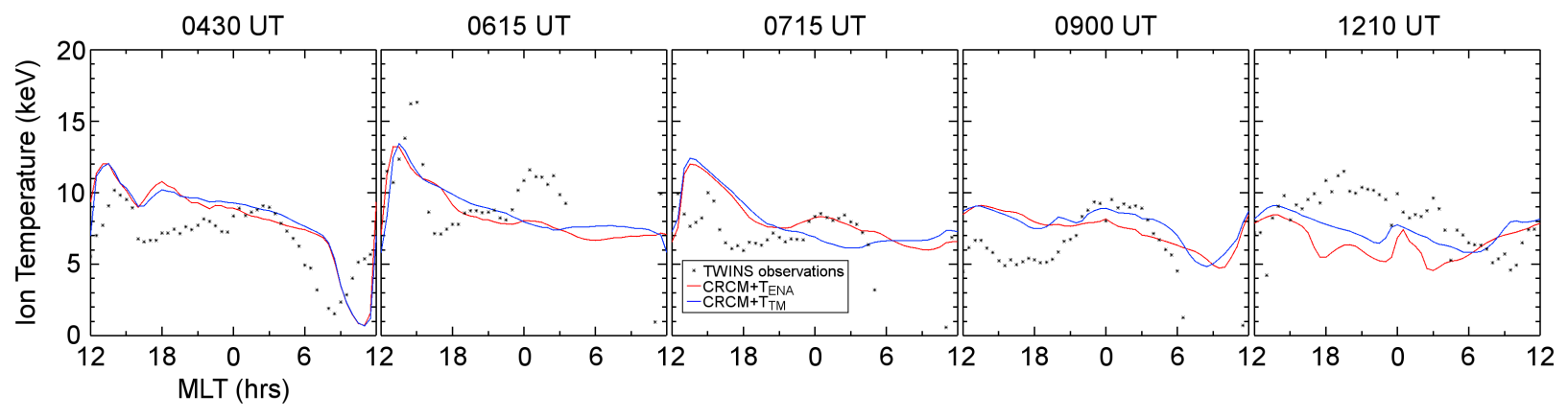

Figure 4.15: Data-model comparison of temperatures calculated at geosynchronous orbit for each boundary condition model. The red (blue) trace corresponds to the ENAderived (TM) boundary condition model. The black data represent geosynchronous temperatures obtained from ENA-derived temperature maps.

elementary drift theory (see e.g., Garner ${ }^{25}$ ) and with the observations in Zhang et al. ${ }^{24}$ and Wing et al. ${ }^{26}$ During the 0430-0530 UT period, recall that the IMF was southward and thus convection was near its strongest of the event, and also that $T_{T M}=12 \mathrm{keV}, T_{E N A}=7 \mathrm{keV}$, so it is natural to expect the temperatures at geosynchronous orbit, $T_{T M \text {,geo }}$ and $T_{E N A \text {,geo }}$ to reflect what is convecting in from the plasma sheet. At 0430 UT (Figure 4.15a), we find that the $T_{T M}$ run (blue) produces slightly higher nightside temperatures, $T_{T M, g e o}$, than the $T_{E N A}$ run. The simulated temperatures are in good general agreement with the Zhang et al. results, as 0430 UT was near the zero epoch time used in their study. Both simulations also show localized, hot populations (peaks) in the dusk-midnight quadrant. This peak is not obvious in the TWINS observations in the dusk sector, but those observations do show a localized hot peak near 3 MLT. Here, both simulations produce a dusk-dawn asymmetry that is oppositely directed than the TWINS observations. Keesee et al. ${ }^{13}$ demonstrated that this hot structure, which is found in the TWINS observations (Figure 4.15a), in fact convected across midnight from dusk and may be associated with a substorm expansion from 00000400 UT. Denton and Borovsky ${ }^{15}$ also identified this as a feature which is characteristic of 
HSS-driven events shortly after the time of convection onset. Overall, the $T_{E N A}$ run shows a slightly cooler $T_{E N A, g e o}$ at 0615 UT than at $0430 \mathrm{UT}$, which is particularly evident near dusk. Unfortunately, the dawn sector was not within the TWINS field of view during this time, hence the absence of dawn-side data (black) in Figure 4.15b. Note the observed hot patch from 0-2 MLT, which is also found, more subtly, in the simulated temperature using $T_{E N A}$ boundary conditions. At the end of the ensuing northward phase, Figure $4.15 \mathrm{c}$ shows that the $T_{T M, g e o}$ are significantly cooler than those found during the preceding southward phase (Figure 4.15a). This is true for the $T_{E N A, g e o}$ as well, with the exception of a weakly localized hot patch found in the midnight sector. This hot patch, also found at 0615 UT, agrees nicely with the TWINS observations as the $T_{T M}$ simulation produces the coolest temperatures in this region. In the pre-midnight sector, the $T_{E N A}$ simulation again agrees more closely with the TWINS observations in that the ion temperature curve is roughly constant from dusk to midnight. The magnitudes are not in perfect agreement, but the spatial distribution in this quadrant is reproduced well by the $T_{E N A}$ simulation. During the interval of southward IMF from 0715-0900 UT, both boundary condition models show nightside heating (Figure 4.15d), but where that heating occurs in MLT depends on the boundary condition model. The $T_{E N A, g e o}$ near midnight remain roughly the same as at 0715 UT, but temperatures increase slightly near dusk which gives a temperature versus MLT picture that is similar to the earlier southward phase (Figure 4.15a). During this southward interval the $T_{T M}$ run produces significant cooling in the dawn sector as well as significant heating around midnight. The observed temperature magnitudes near midnight are matched well by the $T_{T M}$ model, and geosynchronous temperatures from $T_{T M}$ agree better in terms of how the temperature changes in MLT, although both simulations seem to correctly capture the cold $\vec{E} \times \vec{B}$ drifting populations found convecting to the dayside via dawn. Later in the 
recovery phase, at $1210 \mathrm{UT}$ (Figure 4.15e), $T_{E N A, g e o}$ are largely similar to $T_{E N A, g e o}$ at 0900 UT, with a cooler population in the midnight-dawn sector. During the interval 0900-1200 UT, the temperature boundary conditions decrease significantly in both models, which is ultimately reflected in the calculated ion temperatures at geosynchronous orbit during that time. When the $T_{E N A}$ increases abruptly at $1210 \mathrm{UT}$, the impact at geosynchronous orbit is small because the convection field is very weak at that time. Both boundary condition models produce temperatures that approximately agree with the TWINS observations in that the dusk-dawn asymmetry is reproduced, although the pre-midnight $T_{E N A}$ model results better match the warm bump in the observed temperatures.
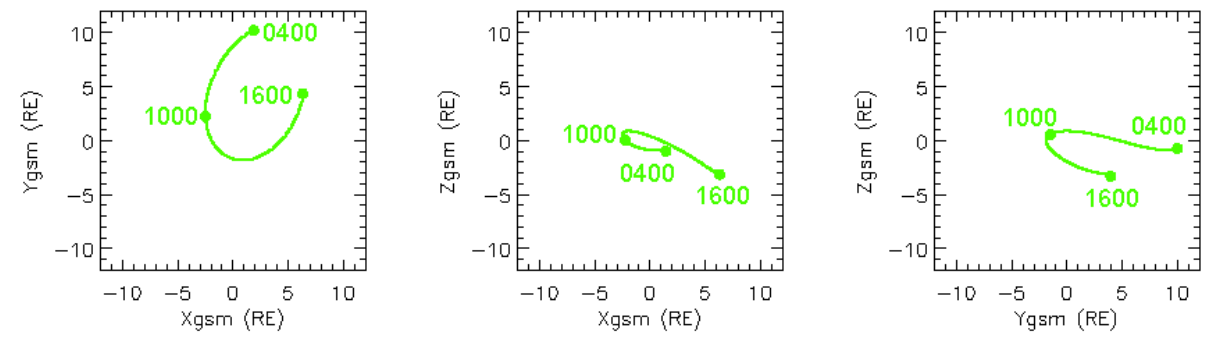

Figure 4.16: THEMIS-D orbit plots ( $\mathrm{x}-\mathrm{y}, \mathrm{x}-\mathrm{z}, \mathrm{y}-\mathrm{z}$ ) in GSM coordinates for 0400-1600 UT during the 22 Jul 2009 event.

In addition to the comparison with remotely imaged observations by TWINS, we also present a comparison of our simulation results with in situ data. THEMIS-D and THEMIS-E (TH-D, TH-E) both sampled the inner magnetosphere during subsets of this 24-hour period. First the focus is on results from TH-D, and comparisons of TH-E data directly follow. THD entered the magnetosphere through the dusk magnetopause flank at 0400 UT, passing through the storm-time ring current in the pre-midnight sector, reaching geosynchronous orbit (19 MLT) at $0810 \mathrm{UT}$, crossing the midnight meridian around $1055 \mathrm{UT}$ at $L=2$, and passing through the pre-noon ring current during the recovery phase. Orbit plots for TH-D 
in the $\mathrm{x}-\mathrm{y}, \mathrm{x}-\mathrm{z}$, and $\mathrm{y}-\mathrm{z}$ planes (all in GSM coordinates) are shown in Figure 4.16.

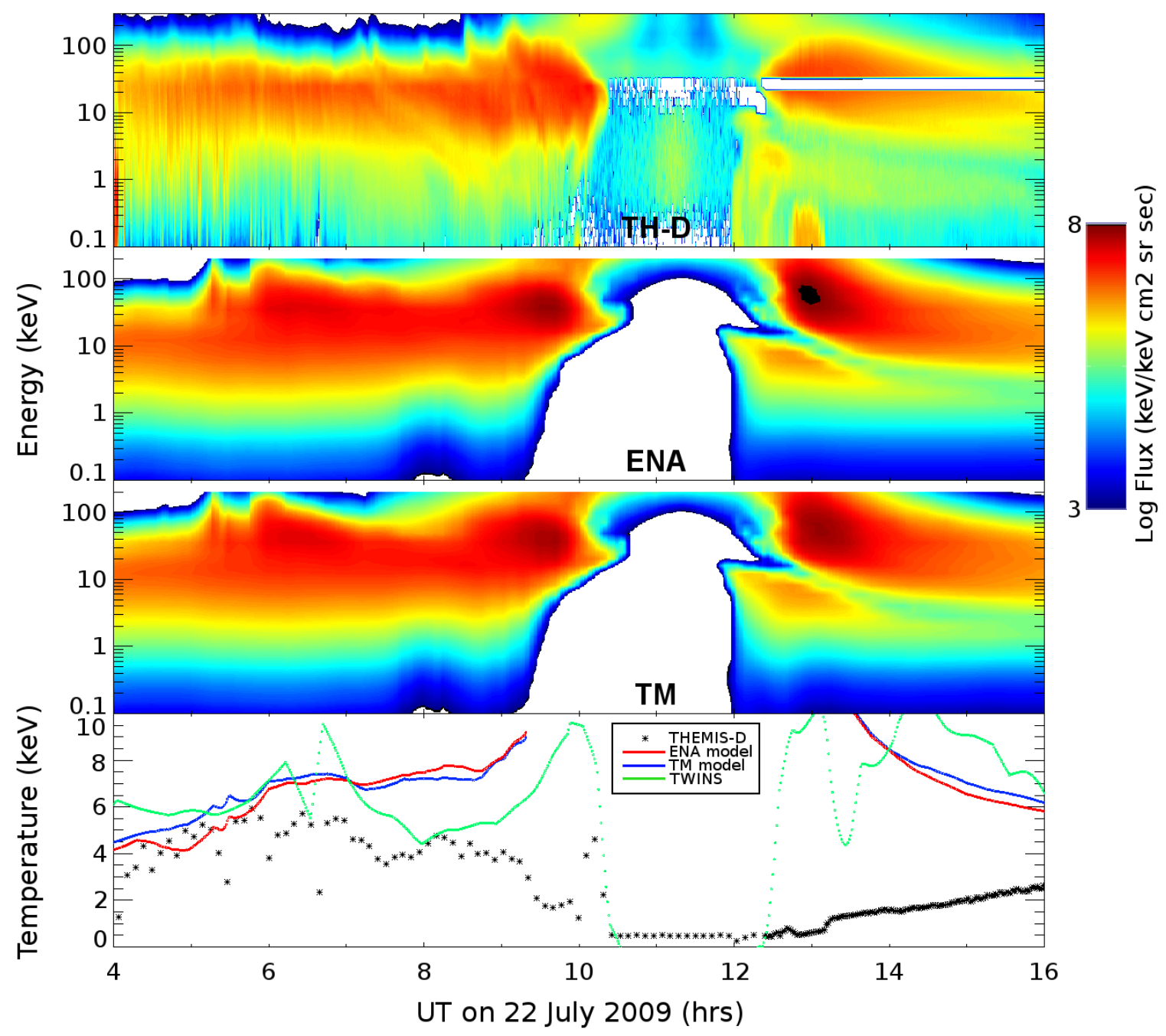

Figure 4.17: Observed (top) and simulated (middle two) THEMIS-D spectrograms for 22 Jul 2009 covering the 0400-1600 UT interval. Top panel shows the combined spectrogram from ESA+SST data. Middle panels show simulated spectrograms from CRCM simulations using the ENA and TM boundary condition models. Bottom panel shows observed and simulated temperature moments. Black data are temperatures provided by TDAS, as observed by THEMIS-D. The green data are observed temperatures extracted from TWINS 2-D temperature maps. Red (blue) data are simulated temperatures using the ENA (TM) boundary condition model.

The top panel of Figure 4.17 shows the TH-D energy flux spectrogram generated by combining background subtracted measurements from the ESA instrument with SST instru- 
ment measurements which have sun contamination removed. The second and third panels of Figure 4.17 are simulated TH-D spectrograms generated from CRCM fluxes produced by the $T_{E N A}$ and $T_{T M}$ boundary condition models respectively. The bottom panel of Figure 4.17 shows the data-model comparison of ion temperature moments computed over the energy range of the ESA instrument (0-25 keV for ions). The black data show the TH-D temperature moment provided through TDAS, the red (blue) data are temperatures computed by applying Equation 2.17 to CRCM fluxes produced using the $T_{E N A}\left(T_{T M}\right)$ boundary condition model, and the green data show the temperatures extracted from TWINS ENAderived maps (see Figures 4.2-4.7). Overall, both simulations reproduce the major features of the ring current observed by the TH-D spacecraft during this time interval. The simulated TH-D temperatures generally agree with the observed temperature, although the omission of a plasmasphere module in our simulations means that cold plasmaspheric populations $(\sim 1 \mathrm{eV})^{27}$ at low $L$-shells are not captured in the CRCM simulations. This causes the ion temperature moments to diverge when TH-D is deep in the inner ring current (0900-1300 $\mathrm{UT})$.

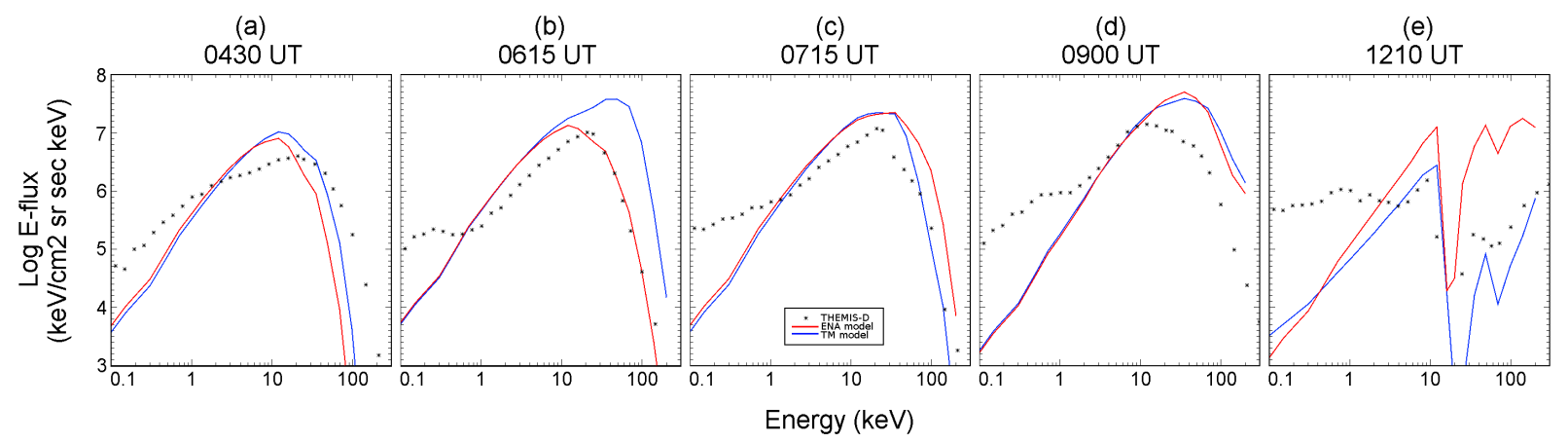

Figure 4.18: Observed and simulated THEMIS-D energy spectra at selected times. Black data are ESA+SST measurements from TH-D, and red (blue) data correspond to simulated TH-D spectra using the ENA (TM) boundary condition model.

From 0415-0545 UT, the $T_{T M}$ produces ion temperatures roughly $20 \%$ higher than those 
from the $T_{E N A}$ model. Note that TH-D was located near the dusk meridian magnetopause boundary at $0415 \mathrm{UT}$, and that the $T_{T M}$ boundary conditions were hotter during this interval (see Figure 4.8). As TH-D was not within the magnetosphere prior to about 0400 UT, the spacecraft was unable to detect any effects associated with the earlier substorms. During the 0415-0545 UT interval, the southward orientation of the IMF enhanced convection from the plasma sheet, and the hotter $T_{T M}$ boundary conditions result in higher fluxes in the high-energy range. This feature is evidenced in Figure 4.18a, which shows a comparison of observed and simulated energy spectra at 0430 UT. The simulated spectra agree with one another below $10 \mathrm{keV}$, but fluxes above $10 \mathrm{keV}$ from the $T_{E N A}$ boundary condition model drop off at lower energies than fluxes from the $T_{T M}$ model. The simulated fluxes differ, roughly, by an order of magnitude in the 20-100 keV energy range. In addition, neither model represents the extent of the high-energy tail found in the TH-D data at this time. As TH-D moves through the ring current to lower $L$-shells and towards the midnight meridian, a steady increase in observed and simulated temperatures is found (Figure 4.17, bottom panel). By $0615 \mathrm{UT}$, the $T_{E N A}$ and $T_{T M}$ model yield similar temperatures $\left(7 \mathrm{keV}\right.$ for $T_{T M}$, $6.5 \mathrm{keV}$ for $T_{E N A}$ ), while the TH-D moment gives a local ion temperature of $4.8 \mathrm{keV}$. Figure 4.18b shows the energy spectra at 0615 UT; as before, both simulations give nearly identical spectra in the low energy $(<10 \mathrm{keV})$ range. However the $T_{E N A}$ model agrees identically with the high-energy tail found in the TH-D SST data, while the $T_{T M}$ model fluxes are a factor of 10-100 higher than the observations. At 0715 UT, the end of the northward interval, a dip in the simulated and observed temperatures occurs (Figure 4.17, bottom panel), although the decrease in the observed temperature is more pronounced. TH-D was in the dusk-midnight quadrant at this time and the preceding $T_{E N A}$ boundary conditions were considerably hotter, and thus the simulated high energy flux is expected to be higher in the dusk quadrant for 
the $T_{E N A}$ run. As shown in Figure $4.18 \mathrm{c}$, the two simulations agree identically up to 30 $\mathrm{keV}$ at $0715 \mathrm{UT}$. However the $T_{E N A}$ model produces a high-energy enhancement, which is consistent with our expectations. Note that during the time frame 0715-0900 UT, THEMIS was near geosynchronous orbit (19 MLT) and observed constant temperatures (4 keV) during this interval. In this regard, the TWINS and TH-D data are in good agreement (see Figure $4.15 \mathrm{c}, 4.15 \mathrm{~d}$ ) as TWINS also provides constant $5 \mathrm{keV}$ temperatures in this region.

The post-midnight substorms at 0829 UT (1.1 MLT) and 0854 UT (1.6 MLT) are manifest as a dispersive negative energy slope in the TH-D energy-time spectrogram from 0800-0900 UT (Figure 4.17, top), as emphasized and discussed by Fok et al. ${ }^{6}$ This negative slope is commonly observed for post-midnight injections that occur when a spacecraft is located in the pre-midnight sector. Energetic ions gradient-curvature drift duskward, with high-energy ions arriving at the detector location before lower-energy ions. ${ }^{6}$ The negative slope feature is reasonably reproduced by both boundary condition models, most visibly in the $<3 \mathrm{keV}$ range (Figure 4.17, middle panels). Although these CRCM simulations do not include a self-consistent magnetic field, and thus cannot reproduce complete substorm physics, the increase in boundary temperatures for both models near 0800 UT (Figure 4.8) serves to approximate the post-midnight injection. In the bottom panel observations, both the TH-D and TWINS temperatures appear to respond to the substorm activity in this time interval. The TWINS temperatures show a roughly linear increase in this region, while the TH-D moments show a hot patch seemingly superimposed on a $<4 \mathrm{keV}$ background. While the $T_{T M}$ simulation gives a constant temperature over this interval, the $T_{E N A}$ model results show a local increase in ion temperature; as this relative change in local ion temperature is better modeled in the $T_{E N A}$ run, it is apparent that the TWINS observations provide more realistic boundary conditions for this interval. 
At $0900 \mathrm{UT}$, the nightside boundary conditions are roughly equal, and thus produce similar energy spectra across the entire energy range as shown in Figure 4.18d. Above 10 keV, the magnitudes of the simulated spectra do not agree well with observations. After 0900 UT, TH-D moves to lower $L$-shells through the post-midnight inner ring current edge and crosses the inner edge on its outbound pass beginning around 1200 UT. Another spectral signature found in the THEMIS spectrogram is a type of ion spectral gap (ISG) frequently called a drift hole. McIlwain ${ }^{28}$ explained this feature as being caused at geosynchronous orbit by enhanced proton drift times due to oppositely directed $\vec{E} \times \vec{B}$ and gradient-curvature drifts for ions within a narrow energy band. The affected energy range, wherein an ion flux dropout occurs, is a function of ion location as well since the particle energy changes to conserve the first adiabatic invariant. Shirai et al. ${ }^{29}$ provided additional insight with Akebono observations of ion flux dropouts from a polar orbit configuration. Thus those measurements sampled a smaller portion of the pitch angle distribution when at high latitudes. Buzulukova et al. ${ }^{30}$ examined the effect of the convection electric field model on the resulting ISGs found in energy-time spectrograms using Interball-2 observations and confirmed the mechanism proposed by McIlwain.

Both simulations reproduce the 10-20 keV drift hole encountered from 1200-1300 UT during this outbound pass in the dawn-noon quadrant. The observed and simulated energy spectra associated with this drift hole are shown in Figure 4.18e. The 'bottom' of the drift hole $(20 \mathrm{keV})$ in the $T_{E N A}$ run agrees better with the observed value, but the $T_{T M}$ model does well in reproducing the 5-10 keV and 30-200 keV energy spectra. Around 1300 UT, TH-D is near noon at $L=4$, sampling the core of the dayside ring current. Here the $T_{E N A}$ model produces higher peak fluxes than the $T_{T M}$ model (Figure 4.17), slightly higher than the colorbar maximum. This is a consequence of the concurrent, abrupt increase 
of boundary ion temperatures in the $T_{E N A}$ model. Unfortunately the magnitudes of the simulated temperatures are unphysical during this time (Figure 4.17, bottom panel), and no direct comparison can be made with the TH-D data.

THEMIS-E traveled along an identical orbit as TH-D, although TH-E lagged in time by about 1 hour. TH-E crossed into the magnetosphere through the duskside flank just before 0500 UT, passing through geosynchronous orbit (19 MLT) around 0715 UT, and later passed through the pre-midnight ring current en route to the distance of closest approach (3 MLT) at 1200 UT. TH-E then passed through the inner ring current edge as it moved sunward across the dawn meridian, and later passed through the core of the dayside ring current and exited the magnetosphere in the pre-dusk flank just after 1800 UT. Figure 4.19 shows the $\mathrm{x}-\mathrm{y}, \mathrm{x}-\mathrm{z}$, and $\mathrm{y}-\mathrm{z}$ projections of the TH-E orbit from 0500-1800 UT.
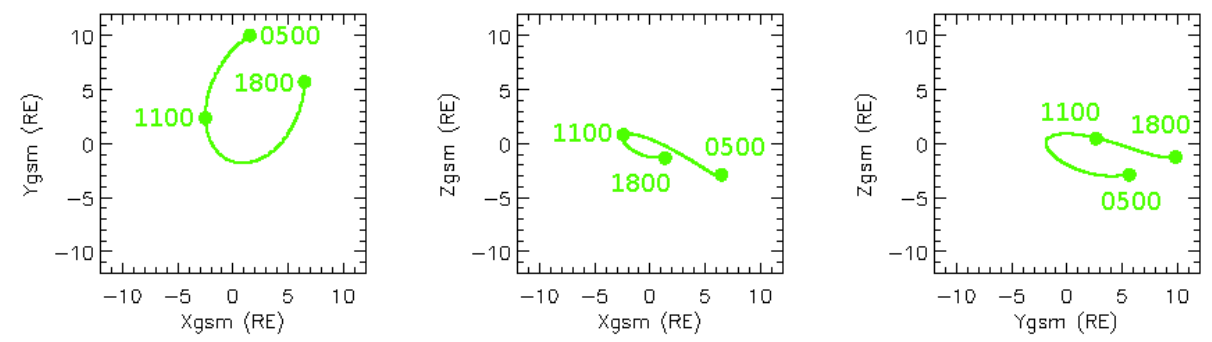

Figure 4.19: THEMIS-E orbit plots ( $\mathrm{x}-\mathrm{y}, \mathrm{x}-\mathrm{z}, \mathrm{y}-\mathrm{z})$ in GSM coordinates for $0500-1800$ UT during the 22 Jul 2009 event. 


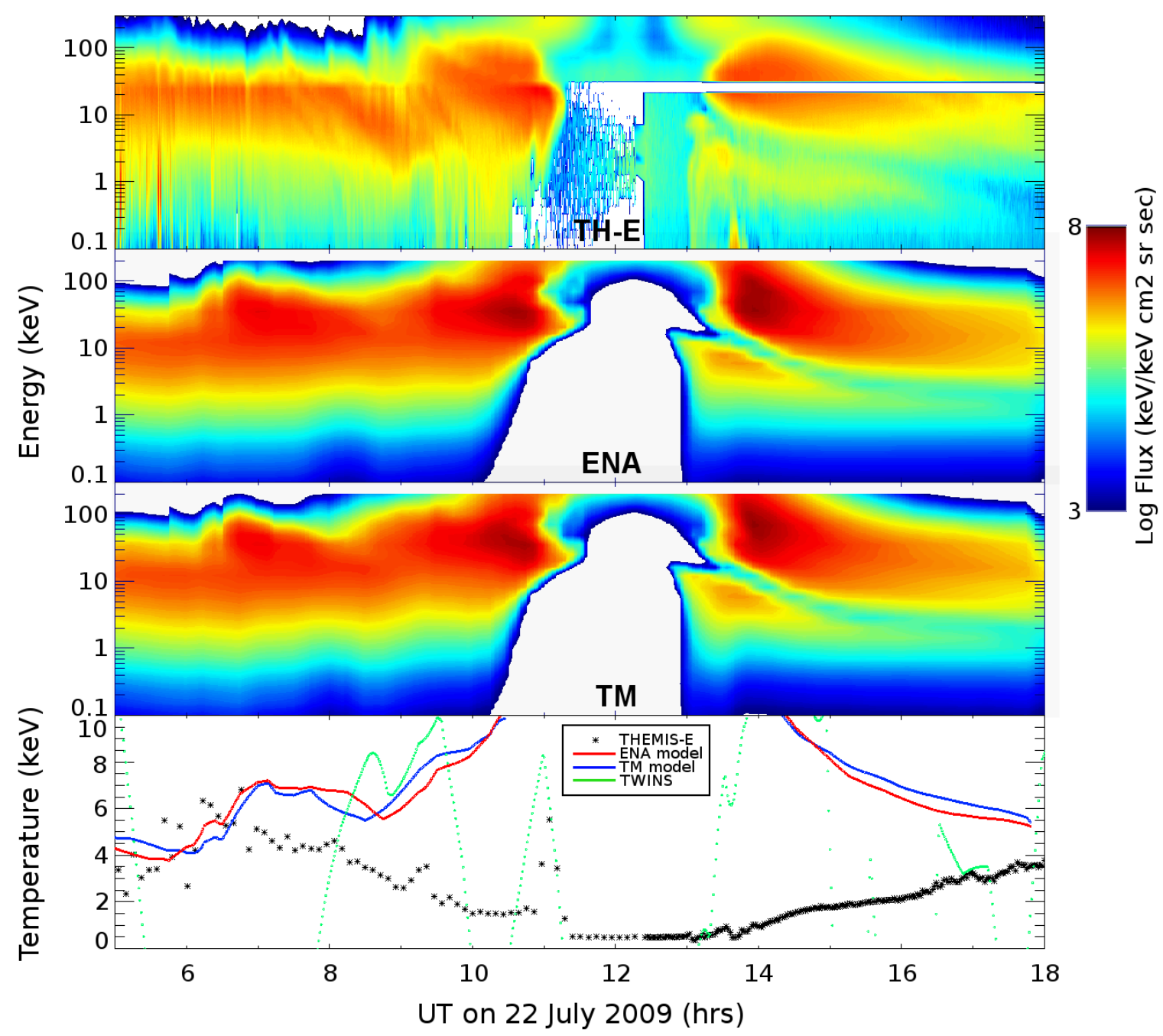

Figure 4.20: THEMIS-E spectrograms and temperature moment comparison. Top panel shows the combined spectrogram from ESA+SST data. Middle panels show simulated spectrograms from CRCM simulations using the ENA and TM boundary condition models. Bottom panel shows observed and simulated temperature moments. Black data are temperatures provided by TDAS, as observed THEMIS-E. The green data are observed temperatures extracted from TWINS 2-D temperature maps. Red (blue) data are simulated temperatures using the ENA (TM) boundary condition model.

Figure 4.20 shows the observed TH-E energy-time spectrogram (top), simulated spectrograms generated from CRCM fluxes using the $T_{E N A}$ and $T_{T M}$ boundary conditions (middle panels), and temperature moments for TH-E and TWINS compared to the simulated temperatures (bottom). The energy ranges contributing to the temperature moments are the 
same as those used in the TH-D discussion (see discussion of Figure 4.17). Overall, the TH-E energy-time spectrogram is similar to that of TH-D (Figure 4.17), but TH-E observed slightly lower ring current energy fluxes than TH-D over the course of the event. TH-E was not sampling the nightside ring current protons during the main phase, causing this relative difference between the two spacecraft (compare the top panels of Figures 4.17 and 4.20).

During the inbound pass from 0600-0800 UT, TH-E observed a relatively homogeneous, $\sim 4-5 \mathrm{keV}$ proton population as indicated by the energy flux spectrogram (Figure 4.20, top) and temperature moment (Figure 4.20, bottom). The spectrograms and temperatures generated from CRCM $\mathrm{H}^{+}$fluxes using both sets of boundary conditions are in reasonable agreement with the observations, although CRCM produces higher fluxes than are measured by TH-E. The simulated spectrograms also show a high-energy enhancement near 0630 UT, coinciding with the period of strongest northward IMF, which is not found in the TH-E data. Note that a corresponding enhancement was found when simulating TH-D energy fluxes (see Figure 4.17), which indicates that the enhancement was not highly localized in space. This simulation artifact contributes to the temperature moments, causing the abrupt, sustained increase in CRCM moments starting at 0630 UT. Temperatures from TWINS ENA maps are not available for comparison during this time interval.

By 0800 UT the IMF had turned southward, resulting in the injections observed by the TH-E spacecraft, i.e., the intermittent negative energy slopes in the top panel of Figure 4.20. The hotter $T_{T M}$ boundary conditions associated with the southward IMF result in a high-energy enhancement in the TM model spectrogram, thus giving higher temperatures than those from the $T_{E N A}$ model. The TWINS ENA temperatures fluctuate between $6-8 \mathrm{keV}$ during this time, and are in decent agreement with the CRCM temperature moments. At $0910 \mathrm{UT}$, TH-E is at geosynchronous orbit and sampling cold outer plasmaspheric $\mathrm{H}^{+}$ions 
in addition to hot ring current ions; this drives the overall temperature calculation down, indicated by the steady decrease in the TH-E moment as the spacecraft moves to lower $L$ shells. The distinct, superimposed hot patch in the TH-E data (Figure 4.20, bottom) is most likely associated with the preceding substorm injections in the post-midnight sector. This feature is also simultaneously present in the TH-D data, as discussed earlier and presented in Figure 4.17.

TH-E continues across the midnight meridian and through the post-midnight sector and at 1300 UT reaches the inner ring current edge at $L=2.6,9.3$ MLT. Along the continued duskward outbound pass through the dayside ring current, both simulations produce similar energy-time spectrograms. Both reproduce the observed drift hole through the full recovery phase, although CRCM yields higher sustained fluxes.

\subsection{The 30-31 October 2013 Event}

\subsubsection{Solar Wind Parameters}

The 30-31 Oct 2013 event was a weak to moderate storm which was driven by a weak, transient coronal mass ejection (CME). This analysis will focus on the interval 30 Oct 2013 (0000 UT) - 31 Oct 2013 (1200 UT); the CRCM simulation results discussed in this section cover that same interval. A characteristic sudden, brief increase in Sym-H just before 0600 UT preceded the main phase. This feature is called a sudden storm commencement (SSC), as originally discussed in the hydromagnetic limit by Dessler et al. ${ }^{31}$ These features (SSCs) are often found to precede the main and initial phases in CME-driven geomagnetic storms, and are caused by the arrival of a shock embedded in the solar wind. ${ }^{32}$ The main phase and recovery phase of this event are characterized by alternating intervals of sustained IMF 
$B_{z}$ orientation. Figure 4.21 shows selected 1-minute resolution OMNIWeb data provided by the ACE satellite, with all parameters time-shifted to the bow shock nose. The minimum Sym-H was -57 nT, which occurred at 2320 UT on 30 October; thus the extended main phase, including another earlier dip in Sym-H followed by a brief recovery, lasted nearly 18 hours. After the Sym-H minimum a slow, steady recovery followed under nearly constant solar wind conditions (weakly northward IMF $B_{z}$ ) for the next 12 hours. In Figure 4.21, red vertical bars are overlaid to indicate the storm times that are chosen for analysis.

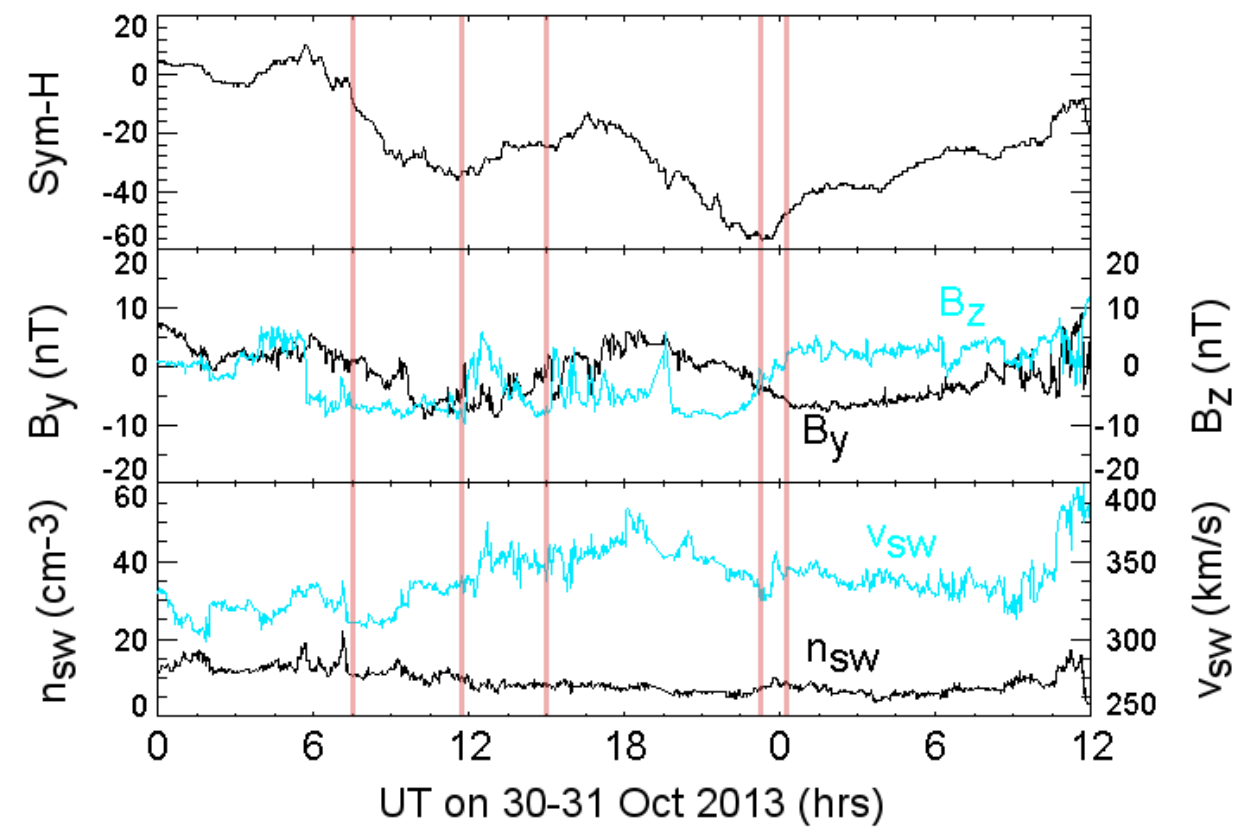

Figure 4.21: OMNIWeb data for the 30-31 Oct 2013 CME-driven geomagnetic storm. Sym-H is shown in the top panel, $B_{y}$ and $B_{z}$ are shown in the center panel, and $n_{s w}$ and $v_{s w}$ are shown in the bottom panel for the 36-hour interval of interest. The red vertical bars indicate times that we select for analysis.

\subsubsection{Storm-time Ion Temperatures}

2-D ion temperatures derived from TWINS 1 and 2 ENA maps are shown in Figures 4.22-

4.26. On average, the ion temperatures provided in these maps are considerably higher than 
those computed for the HSS-driven 22 Jul 2009 event (see Figures 4.2-4.7), with magnetospheric temperatures for this moderate CME-driven storm often averaging $10-15 \mathrm{keV}$. The superposed epoch analysis presented by Keesee et al. ${ }^{33}$ suggests that average inner magnetospheric ion temperatures are higher for moderate $(-78 \mathrm{nT}<\mathrm{Dst}<-41 \mathrm{nT})$ CME-driven storms than for HSS-driven storms in the same Dst range. Denton et al. ${ }^{34}$ also performed a superposed epoch analysis with the intention of discerning how the plasma sheet temperature responds to the two unique drivers (CMEs and HSSs). The authors found that slightly higher plasma sheet ion temperatures are found under the influence of HSS storms, a result in contrast to the Keesee et al. study. Regardless, average global temperatures from both of these studies are in good agreement with the sustained $\approx 10 \mathrm{keV}$ temperatures visible in Figures 4.22-4.26. Therefore, although the 30-31 Oct 2013 storm was rather weak by virtue of the Sym-H index, such elevated ion temperatures are not unexpected, and thus it is useful to understand what effects such high boundary temperatures produce in inner magnetospheric simulations. Ebihara and Ejiri ${ }^{35}$ reported that ring current buildup is insensitive to ion temperature boundary conditions above $3 \mathrm{keV}$, but their numerical simulations used simplified global magnetic and convection field models. The results presented here are in direct contrast to those results, as we find significant differences in the time evolution of the ring current between the two boundary condition models.

Temperature maps covering the pre-storm interval of 0224-0540 UT are shown in Figure 4.22. The average global temperature is found to increase, without a significant corresponding change in solar wind conditions, near 0400 UT. As the ring current flux was very low in this pre-storm phase, it is likely that the counting statistics were poor and thus generated unphysical ion temperatures. Just before $0600 \mathrm{UT}$, the IMF $B_{z}$ turned moderately southward and triggered ring current injections to begin the early main phase. This southward 

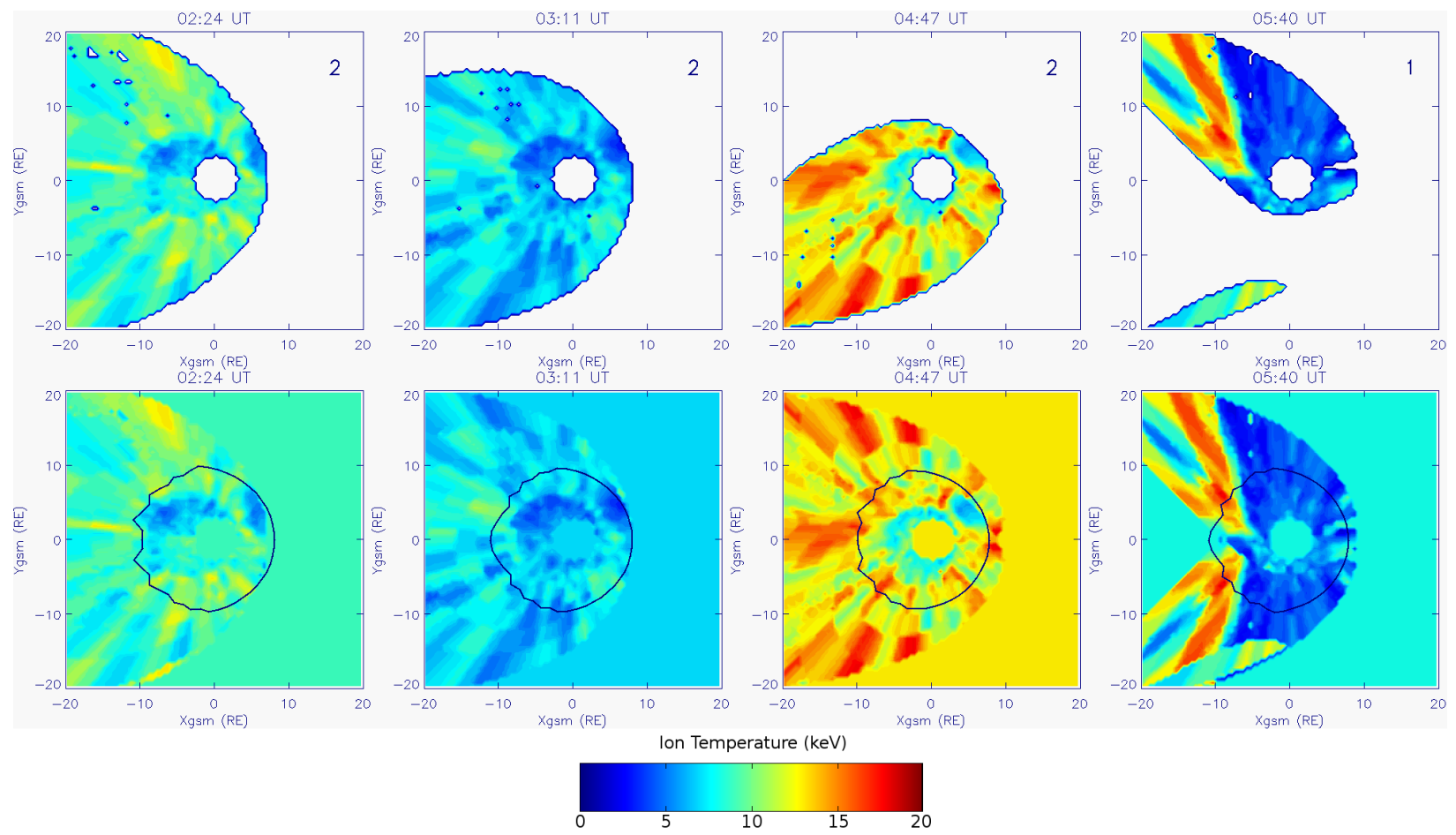

Figure 4.22: ENA-derived equatorial ion temperature maps for 0224-0540 UT on 30 Oct 2013. The top row shows the raw temperature calculations, and the bottom row shows the temperatures used for CRCM boundary conditions; good data are mirrored about the noon-midnight meridian and pixels with missing data are filled with averages. The time-varying CRCM outer boundary is overplotted in the bottom row.

configuration was sustained for about 6 hours; the ENA-based temperature maps for that 6-hour interval are presented in Figure 4.23.

In each panel of Figure 4.23, highly-localized structure is visible in the TWINS 1 maps. In addition, peak ion temperatures of 15-20 keV are considerably higher than expected based on the solar wind conditions and storm phase. Note that some pixels within the TWINS fields of view have no temperatures assigned (top row), as the statistics did not meet the chi-squared minimum. It seems that the temperatures in pixels that neighbor dataless pixels are also unexpectedly high, and thus it is probable that the statistics in these neighboring cells are just above the minimum chi-squared threshold. Regardless of the source of the high 

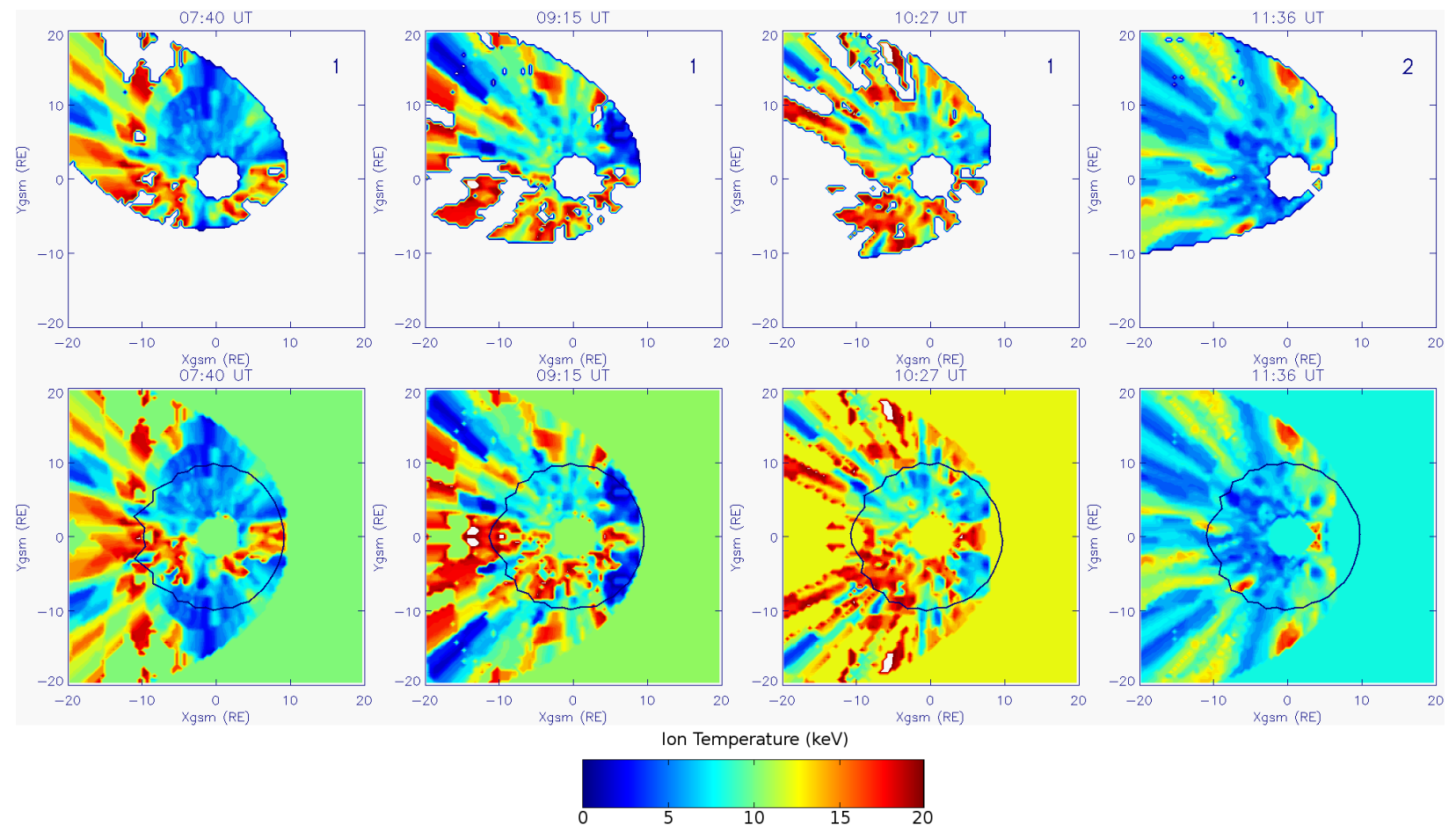

Figure 4.23: ENA-derived equatorial ion temperature maps for $0740-1136$ UT on 30 Oct 2013. The top row shows the raw temperature calculations, and the bottom row shows the temperatures used for CRCM boundary conditions; good data are mirrored about the noon-midnight meridian and pixels with missing data are filled with averages. The time-varying CRCM outer boundary is overplotted in the bottom row.

temperatures, physical or not, the boundary cells which are sampled and supplied to CRCM (the black trace in bottom panels) do capture some of these hot structures and additionally the strong gradients in MLT. Around 1145 UT, the IMF turns northward for a brief interval of $\sim 1$ hour, followed by an interval of weakly alternating IMF $B_{z}$ orientation until 1945 UT. During this interval, the TWINS 2 temperature maps do not provide great coverage of each pixel in the inner magnetosphere, again evidenced in Figure 4.24. The TWINS 2 spacecraft traveled near perigee after about 1600 UT and thus did not permit accurate mapping to the GSM equatorial plane through the neutral exosphere after that time. The Sym-H shows a slow recovery during this interval (see Figure 4.21), until the subsequent southward turning 

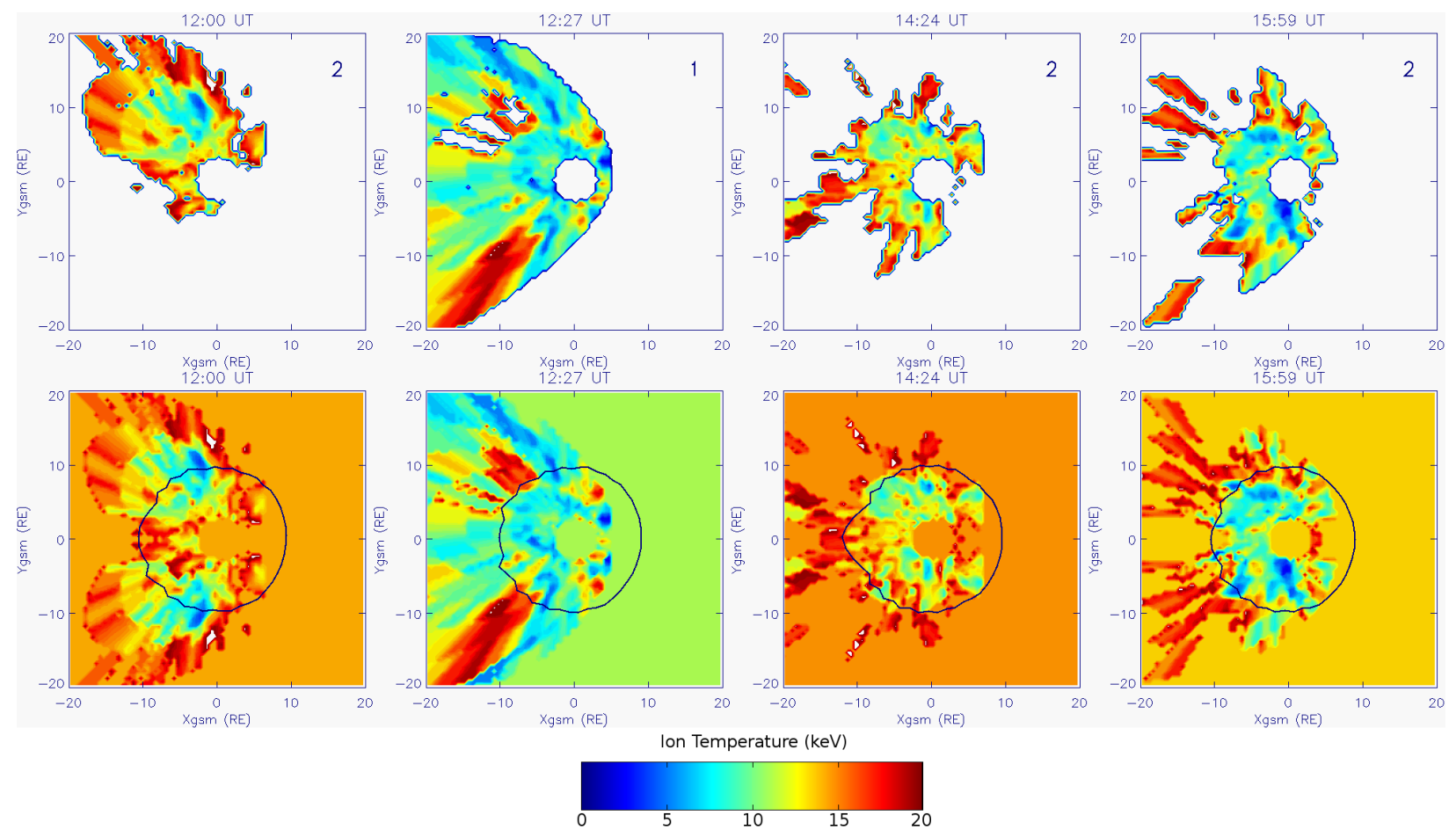

Figure 4.24: ENA-derived equatorial ion temperature maps for 1200-1559 UT on 30 Oct 2013. The top row shows the raw temperature calculations, and the bottom row shows the temperatures used for CRCM boundary conditions; good data are mirrored about the noon-midnight meridian and pixels with missing data are filled with averages. The time-varying CRCM outer boundary is overplotted in the bottom row.

\section{at 1945 UT.}

In Figure 4.25, temperature maps from TWINS 1 are shown for the interval 1738-2049 $\mathrm{UT}$, covering the second interval of decreasing Sym-H. The IMF $B_{z}$ is primarily southward during this time, but some brief northward excursions are also noted in the OMNIWeb data. On average, the TWINS 1 temperature maps during this interval give ion temperatures that are much more consistent with expectations for a geomagnetic event of this magnitude. The TWINS 1 field of view does not cover important nightside regions from 1738-1826 UT, but the duskside portion of the inner magnetosphere that was within view is considerably cooler than in the preceding intervals. As the post-midnight sector comes into the TWINS 1 field 

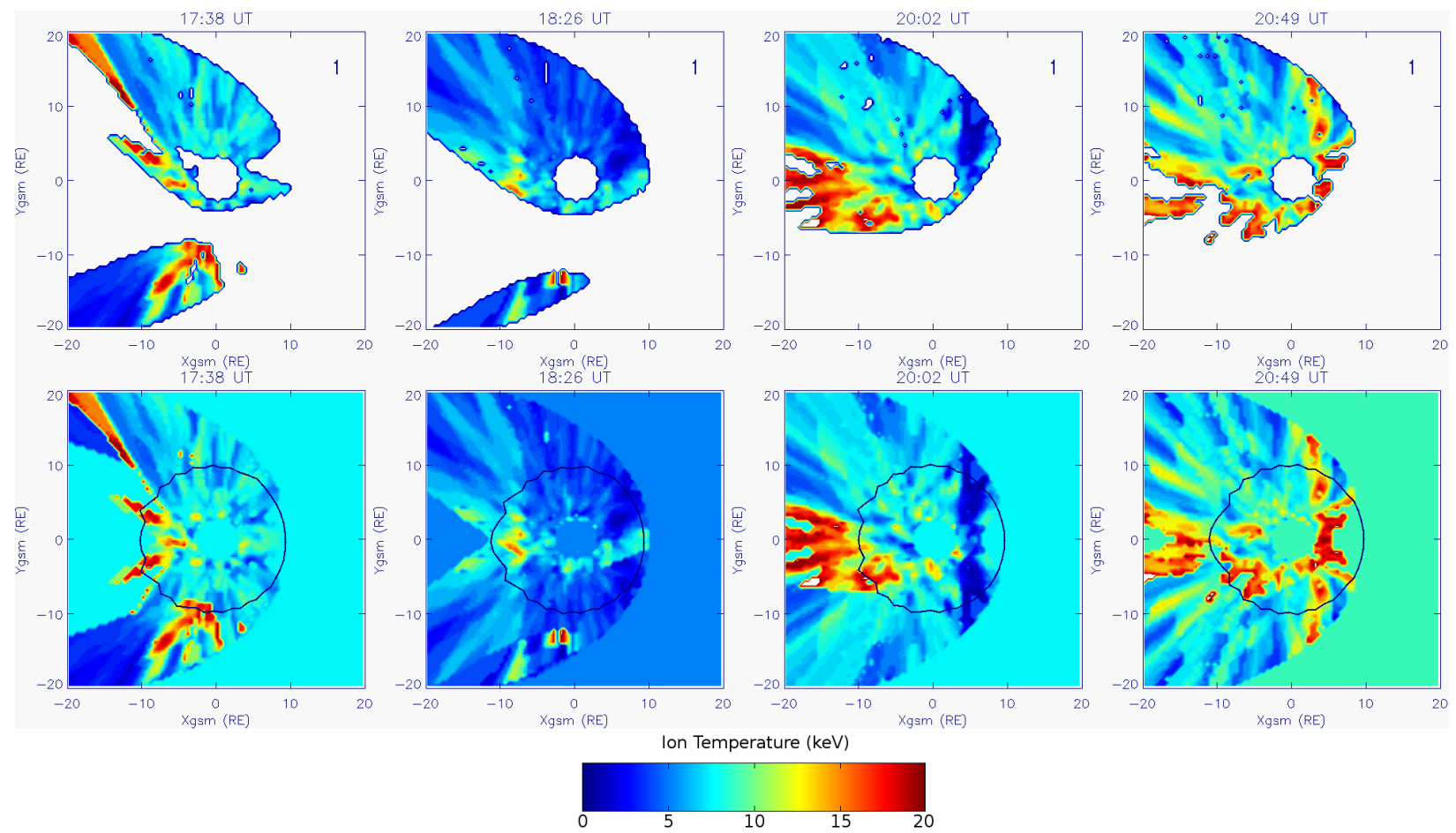

Figure 4.25: ENA-derived equatorial ion temperature maps for 1738-2049 UT on 30 Oct 2013. The top row shows the raw temperature calculations, and the bottom row shows the temperatures used for CRCM boundary conditions; good data are mirrored about the noon-midnight meridian and pixels with missing data are filled with averages. The time-varying CRCM outer boundary is overplotted in black in the bottom row.

of view, around $2000 \mathrm{UT}$, it is found that the post-midnight sector is significantly hotter than the pre-midnight sector, although this effect may be due to instrument head-mismatch, an issue that potentially complicates interpretation of TWINS data (see Section 2.3.1). As TWINS 2 proceeds back out of perigee to higher $L$-shells, the cross-calibration between the two spacecraft is apparent through a visual comparison of TWINS 1 temperatures at 2039 UT (last panel of Figure 4.25) with TWINS 2 temperatures at 2334 UT (first panel of Figure 4.26); ENA-derived ion temperature maps generated independently from both spacecraft are in excellent agreement over this time. After the Sym-H minimum and concurrent northward IMF turning at 0000 UT on 31 Oct 2013, a visible increase in duskside temperatures is 
observed (2600 UT in Figure 4.26).
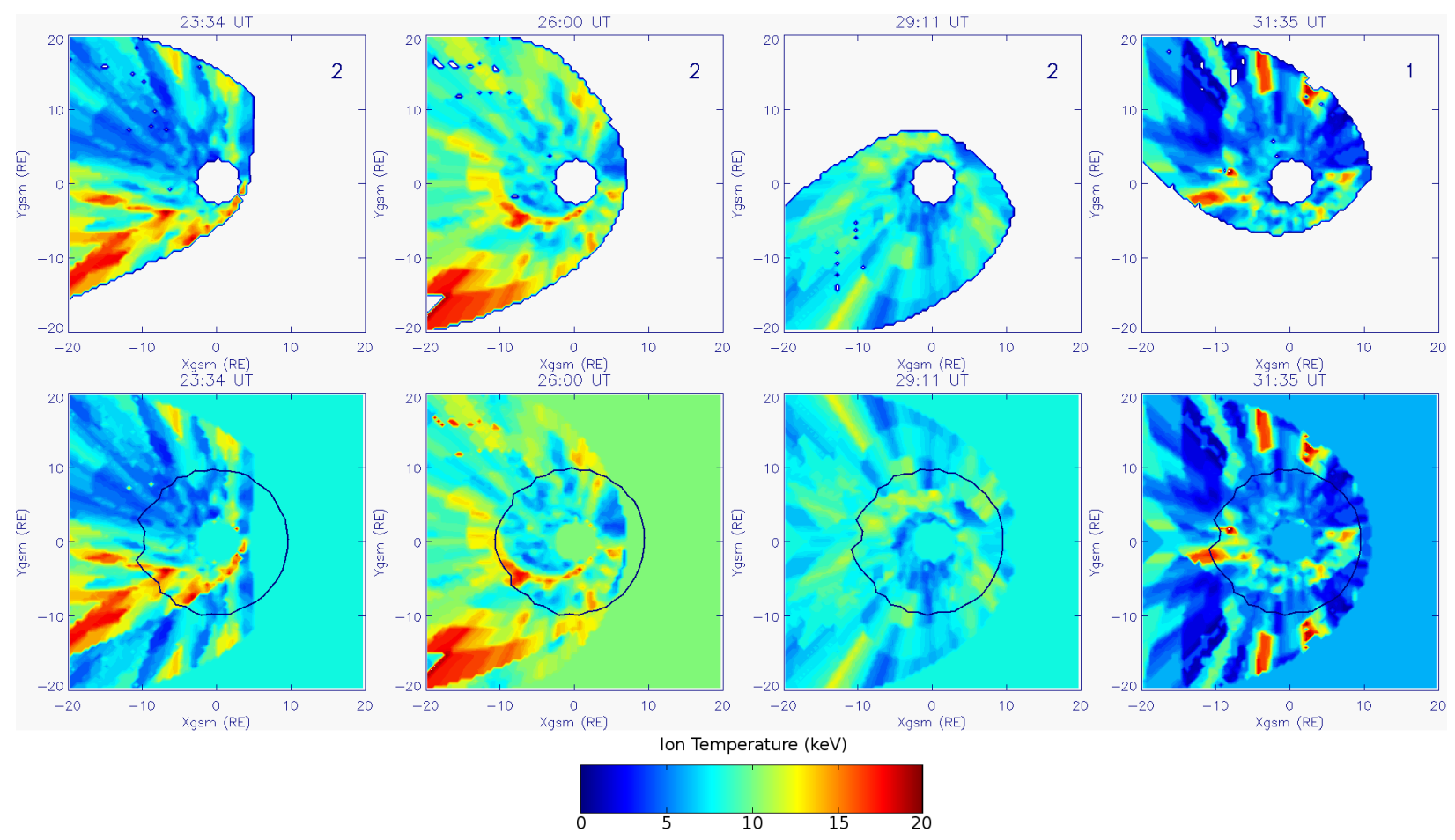

Figure 4.26: ENA-derived equatorial ion temperature maps for 2334-0735 UT on 3031 Oct 2013. The top row shows the raw temperature calculations, and the bottom row shows the temperatures used for CRCM boundary conditions; good data are mirrored about the noon-midnight meridian and pixels with missing data are filled with averages. The time-varying CRCM outer boundary is overplotted in the bottom row.

The ion temperatures sampled at the CRCM outer boundary from the ENA-derived temperature maps in Figures 4.22-4.26 are shown in the bottom panel of Figure 4.27. As a comparison, the ion temperatures provided by the Tsyganenko and Mukai model ${ }^{36}$ are shown in the top panel of Figure 4.27. In general, the global ion temperatures produced by TWINS ENA maps are considerably higher than those given by the Tsyganenko and Mukai model. The TM model temperature boundary conditions at midnight vary only between 6-8 keV. The ENA-derived boundary temperatures are significantly higher for the full 36hour interval, except for short intervals after $0600 \mathrm{UT}, 1800 \mathrm{UT}$ and $3000 \mathrm{UT}$ where the 
temperatures drop to $2-4 \mathrm{keV}$. Outside of these intervals, the TWINS-derived temperatures are generally above $10 \mathrm{keV}$. Contrast this comparison with the 22 July 2009 event study in Section 4.1.2, where on average the two boundary condition models produced very similar temperature ranges (see Figure 4.8). Here we present an analysis of this event, wherein the two boundary condition models give very different ion temperature boundary conditions than the event discussed in Section 4.1.
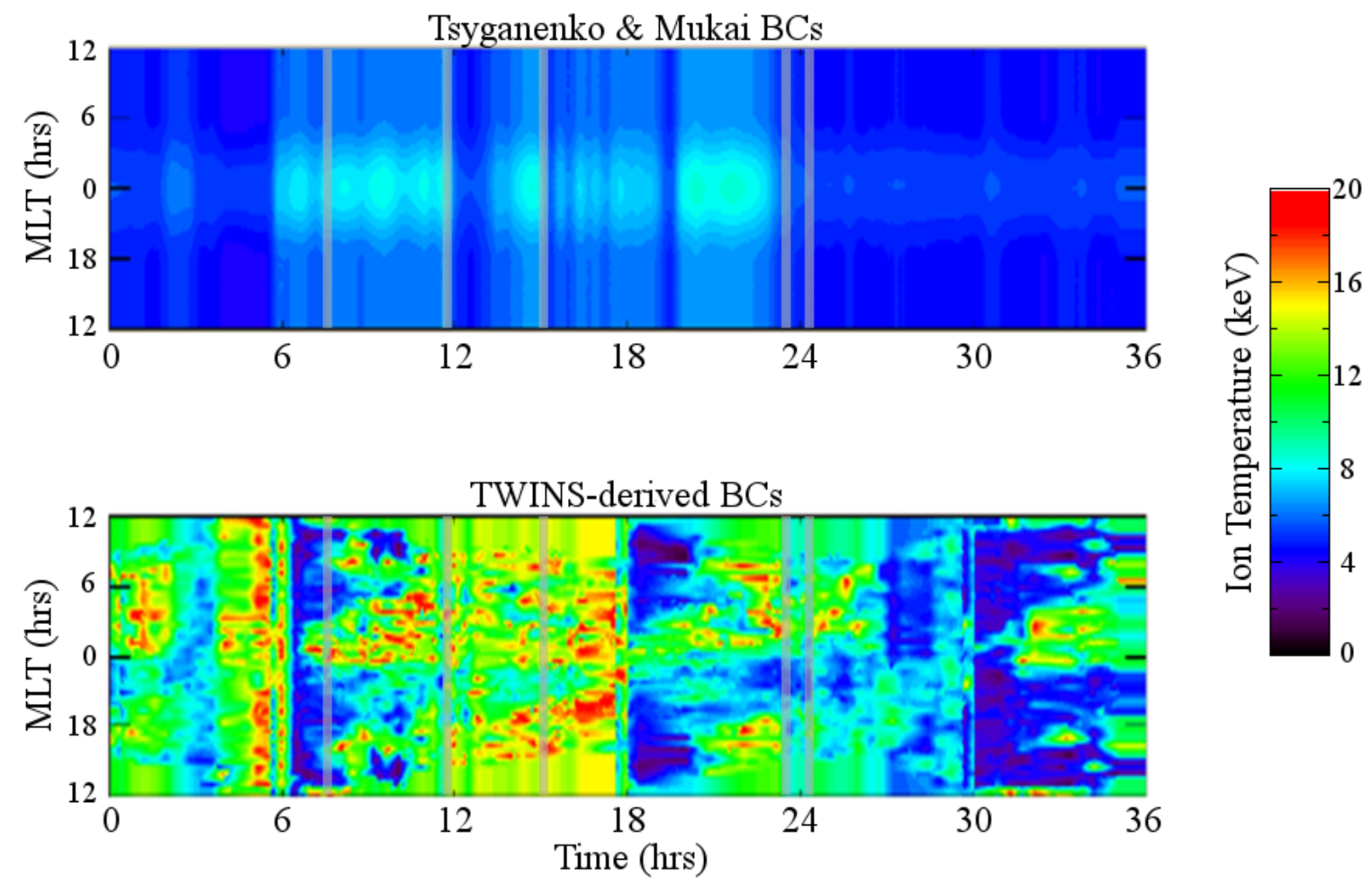

Figure 4.27: Ion temperature boundary conditions for the 30-31 Oct 2013 event. The top panel shows the temperatures provided by the Tsyganenko \& Mukai empirical model, and the bottom panel shows the temperatures calculated using the TWINS ENA data. 


\subsubsection{Simulation Results}

Figure 4.28 shows the simulated $\mathrm{H}^{+}$pressure for both boundary condition models, calculated from the pitch-angle averaged fluxes in Equation 2.20. Simulation results using TM model (ENA-derived) boundary conditions are shown in the top (bottom) row for the five times marked by vertical bars in Figures 4.21 and 4.27. Early in the main phase, the injection from the plasma sheet results in an expected nightside pressure enhancement, most notably in the pre-midnight sector as shown in Figure $4.28 \mathrm{a}$. The peak pressure from the $T_{T M}$ run is found at lower $L$-shells than in the $T_{E N A}$ run, which is due to the colder boundary plasma penetrating deeper into the inner magnetosphere. The localized, hot $\sim 15 \mathrm{keV}$ boundary conditions in the $T_{E N A}$ run produce a pronounced, disparate enhancement just outside of geosynchronous orbit from the dusk meridian to about 21 MLT.

Just before the northward IMF turning at 1200 UT, the Sym-H index shows a local minimum (-36 nT). At $1145 \mathrm{UT}$, the simulated pressure from the $T_{E N A}$ run is considerably more diffuse than in the $T_{T M}$ run (Figure $4.28 \mathrm{~b}$ ). In addition, the peak pressure is somewhat higher in the $T_{T M}$ run and this run also shows a post-midnight enhancement not present in the $T_{E N A}$ run results. All three of these features are expected based on the preceding boundary conditions.

After the subsequent, but brief, northward IMF turning the IMF remains southward until 1500 UT (see Figure 4.21). During the northward interval the inner plasma sheet is loaded with warm, 5-8 keV (hot, 10-16 keV) plasma in the $T_{T M}\left(T_{E N A}\right)$ simulation. Upon the southward turning at $1500 \mathrm{UT}$, the $\mathrm{H}^{+}$pressure reflects the injection of these different plasma populations. Specifically, the $T_{T M}$ run again produces higher peak pressure and the location of that peak is at a lower $L$-shell and more dawnward-shifted than in the $T_{E N A}$ run; peak pressure occurs at $L=4.6,23.5$ MLT using $T_{T M}$ boundary conditions, and at $L=4.9$, 


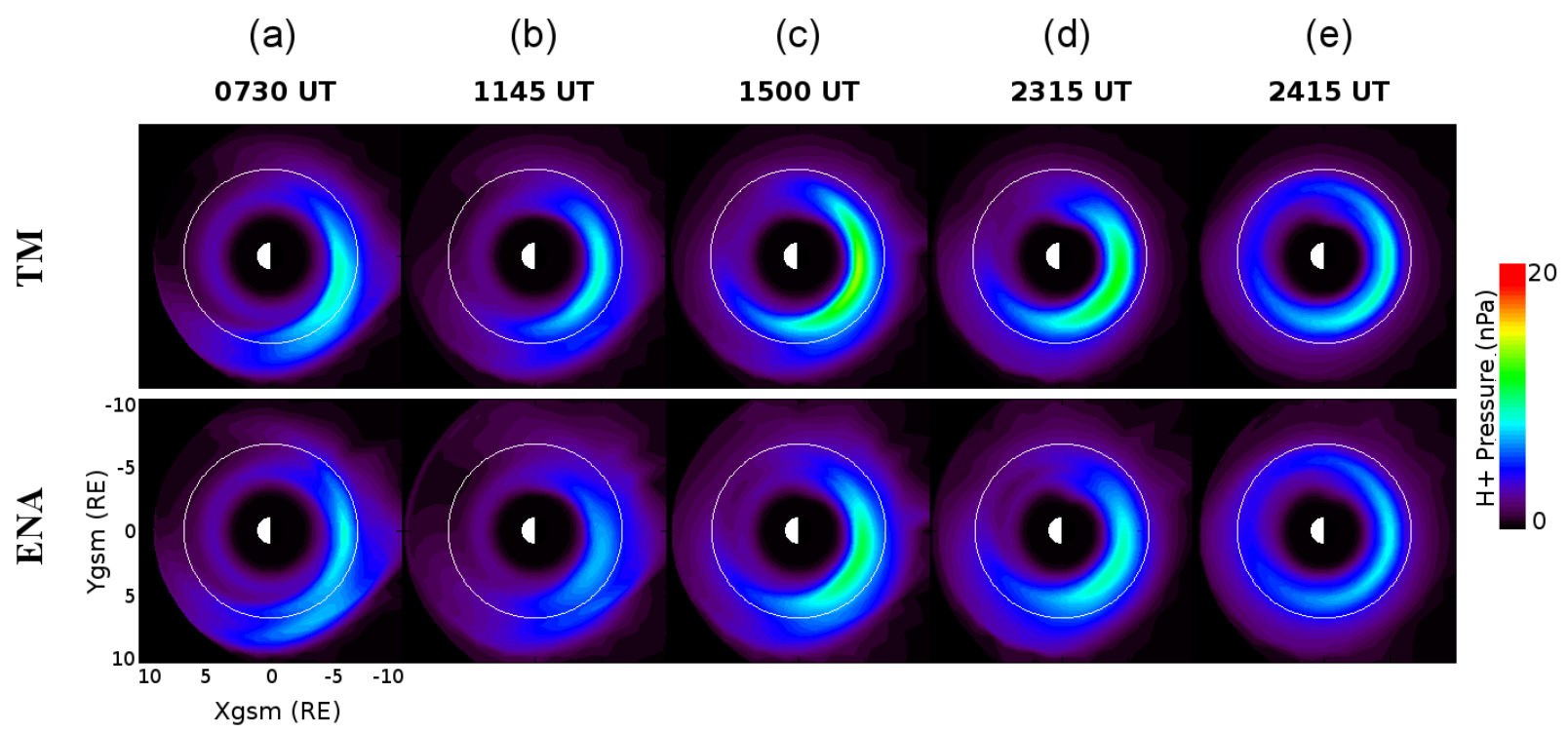

Figure 4.28: $\mathrm{H}^{+}$pressure $(\mathrm{nPa})$ using ion temperature boundary conditions from the TM model (top row), and ENA-derived model (bottom row) at five time steps (columns). Spatial coordinates in GSM are shown at the bottom left panel. In each panel, the sun is to the left and the white traces indicate geosynchronous orbit.

23.0 MLT using $T_{E N A}$ boundary conditions. This signature in the pressure persists through the rest of the main phase, ending with the Sym-H minimum just before 0000 UT on 31 Oct. At the Sym-H minimum (2315 UT, Figure 4.28d) we see evidence of this persisting feature, where the peak pressure remains higher when cooler boundary conditions are supplied.

One hour after the final northward turning (Figure $4.28 \mathrm{e}$ ), the $T_{E N A}$ boundary conditions in the pre-midnight sector are comparable to those provided by the TM model. The ring current begins to close, and the $\mathrm{H}^{+}$pressure starts to become more symmetric in MLT. As the recovery phase continues on, the two boundary condition models produce increasingly similar pressure in terms of both magnitude and spatial distribution.

While considering the storm-time pressure is a well-accepted metric for gauging the inner magnetospheric response during specific events, it is insightful to inspect the contributing distributions in terms of their energy. 6-18 keV (denoted as $12 \mathrm{keV}$ ) and 18-39 keV (28 keV) 
differential fluxes are presented in Figures 4.29 and 4.30, respectively, as the flux in these energy ranges are the primary contributors to total ion pressure.

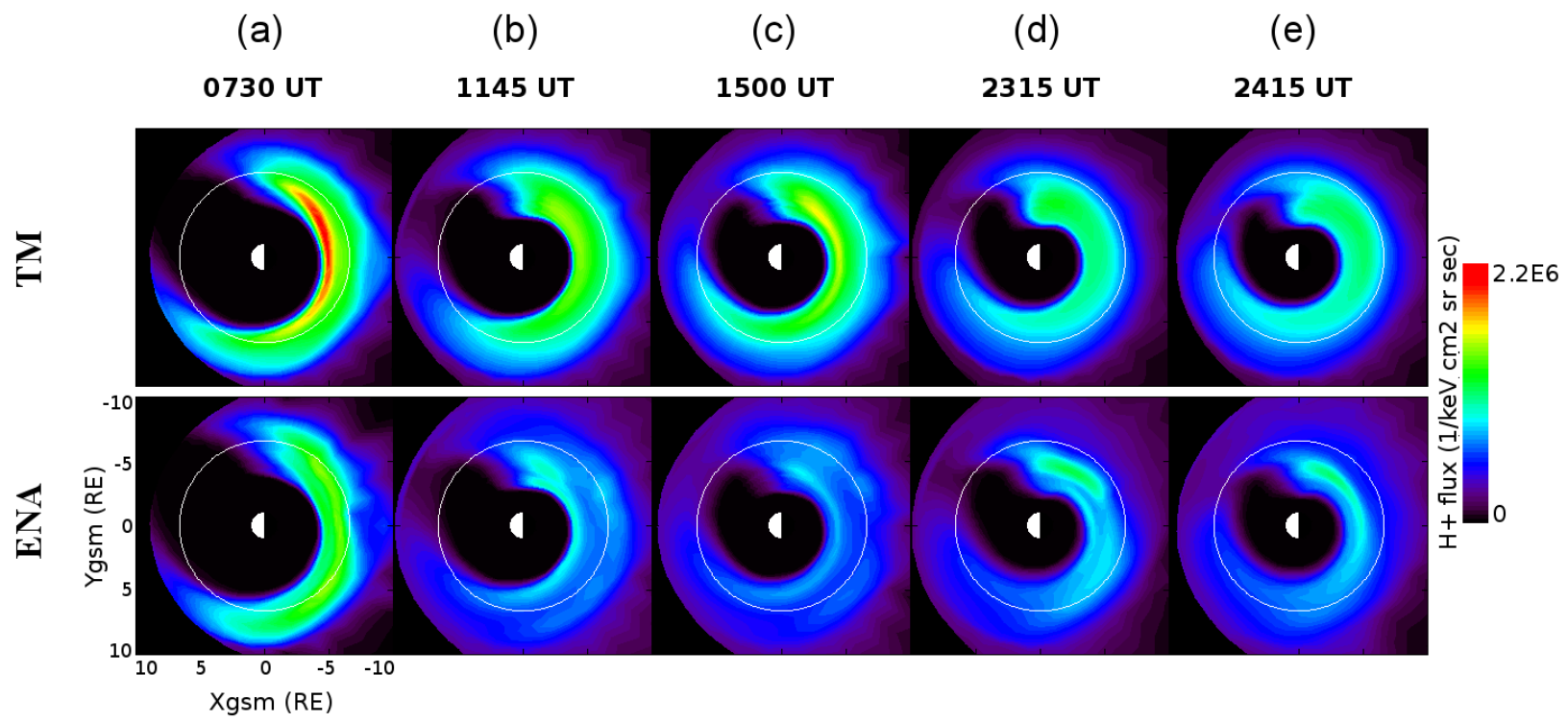

Figure 4.29: Simulated $6-18 \mathrm{keV}(12 \mathrm{keV}) \mathrm{H}^{+}$flux $\left(1 / \mathrm{keV} \mathrm{cm}^{2} \mathrm{sr} \mathrm{sec}\right)$ using ion temperature boundary conditions from the TM model (top row), and ENA-derived model (bottom row) at five time steps (columns). Spatial coordinates in GSM are shown at the bottom left.

Upon examination of the $12 \mathrm{keV}$ fluxes (Figure 4.29), it is obvious that the considerably cooler temperatures provided by the TM empirical model result in significantly enhanced flux in this energy range. In addition, the magnitudes of $28 \mathrm{keV}$ flux (Figure 4.30) are much more similar between boundary condition models. It may be directly inferred that $J(E)$ is increasing with respect to energy over this energy range in the $T_{E N A}$ results, but nearly constant in the $T_{T M}$ results. This indicates that the energy distribution of ion flux, $J(E)$, has a peak near the middle of this energy range in the $T_{T M}$ run, but has a peak at higher energy in the $T_{E N A}$ run. Since, from equation 2.11, $J(E) \sim E e^{-E / k_{B} T}$ for a Maxwellian distribution, the peak occurs where $\frac{d J}{d E}=0$, which occurs at $E=k_{B} T$. Thus the peak flux occurs at higher energy for hotter populations, indicating significantly hotter distributions 


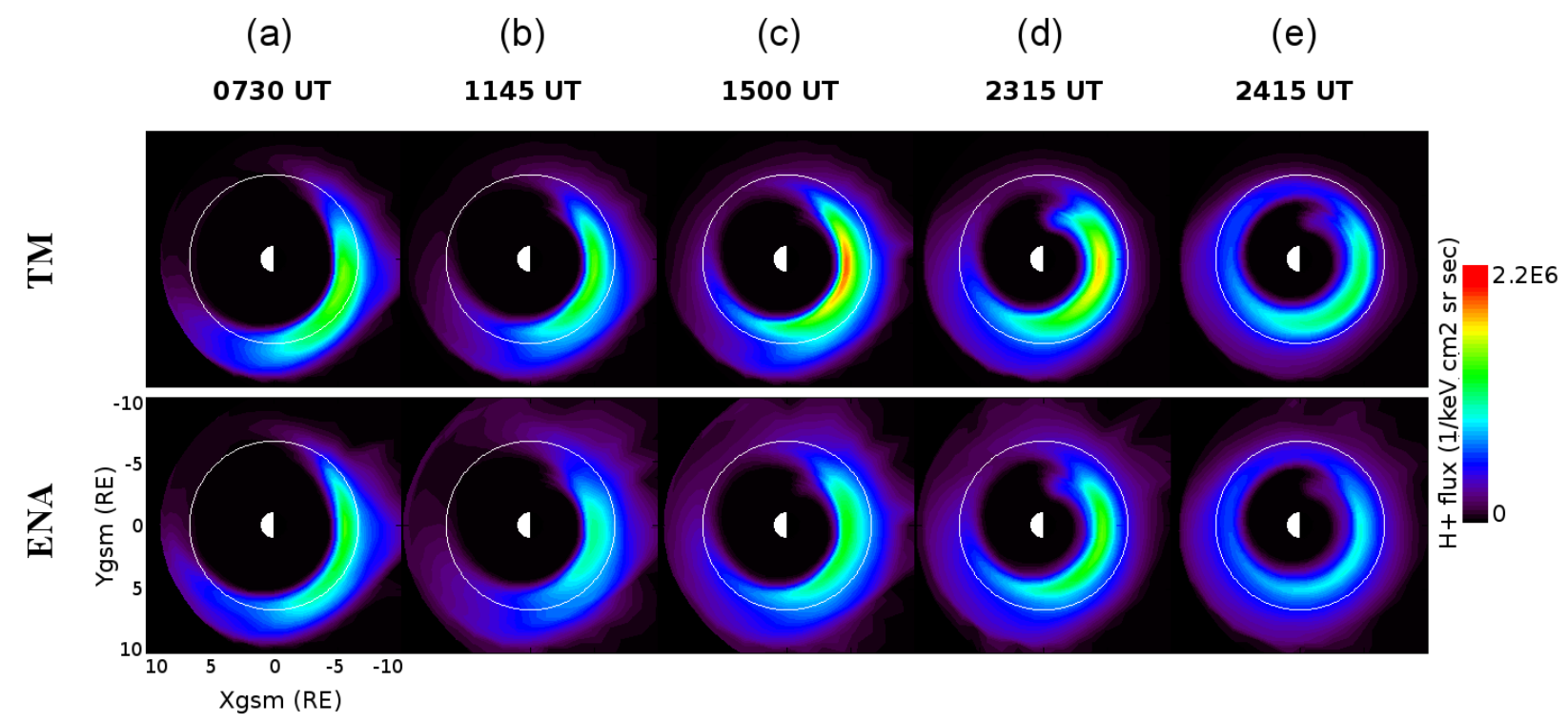

Figure 4.30: Simulated 18-39 $\mathrm{keV}(28 \mathrm{keV}) \mathrm{H}^{+}$flux $\left(1 / \mathrm{keV} \mathrm{cm}^{2} \mathrm{sr} \mathrm{sec}\right)$ using ion temperature boundary conditions from the TM model (top row), and ENA-derived model (bottom row) at five time steps (columns). Spatial coordinates in GSM are shown at the bottom left.

in the $T_{E N A}$ run with the exception of $0730 \mathrm{UT}$.

At $0730 \mathrm{UT}$, shortly after the beginning of the main phase, the $T_{T M}$ and $T_{E N A}$ boundary condition models produce similar $12 \mathrm{keV}$ and $28 \mathrm{keV}$ fluxes (figures 4.29a, 4.30a). The $T_{T M}$ run however shows a $12 \mathrm{keV}$ flux enhancement at the inner ring current edge not found in the $T_{E N A}$ run. The solar wind and IMF parameters remain nearly constant until the brief northward turning at $1145 \mathrm{UT}$; this results in constant $T_{T M} \approx 6-7 \mathrm{keV}$ boundary conditions, whereas the post-midnight $T_{E N A}$ show gradual heating to nearly $20 \mathrm{keV}$ (Figure 4.27). The high $T_{E N A}$ boundary conditions result in a global depletion of $12 \mathrm{keV}$ flux at $1145 \mathrm{UT}$. In fact, the lack of significant $12 \mathrm{keV}$ flux persists throughout the rest of the $T_{E N A}$ simulation (Figures 4.29b-e), due to the consistently hot boundary conditions provided for the full simulation (see Figure 4.27). At $1145 \mathrm{UT}$ the $T_{T M}$ model also provides $28 \mathrm{keV}$ fluxes that are larger in magnitude than those from the $T_{E N A}$ model, although the spatial distributions 
are in agreement. A brief recovery phase follows the northward IMF turning at 1145 UT, followed by a short-lived southward interval which ends at 1500 UT. At 1500 UT, fluxes from both boundary condition models resemble those from the earlier interval of southward IMF, although the magnitudes are slightly higher. The $28 \mathrm{keV}$ flux from the $T_{T M}$ model shows a strong, localized peak near midnight (Figure 4.30c) whereas the $T_{E N A}$ model shows nightside $28 \mathrm{keV}$ fluxes which are roughly constant between $L=4$ and geosynchronous orbit. As the extended main phase continues, the $T_{E N A}$ boundary conditions remain elevated until the brief northward interval just after 1800 UT. During this time, $T_{E N A}$ cool at all MLT until the strong southward IMF again heats the plasma sheet from 2100-2400 UT. The Sym$\mathrm{H}$ minimum occurs at $2315 \mathrm{UT}$, at which time the IMF again turns northward and the $T_{E N A}$ model provides a cooler $\sim 6 \mathrm{keV}$ region in the pre-midnight sector. This cool region subsequently $\vec{E} \times \vec{B}$ drifts towards the dawn sector, resulting in a patchy post-midnight enhancement in the $12 \mathrm{keV}$ flux (Figure 4.29d). The boundary conditions for both models remain the same for the following hour, while the IMF again turns northward and limits convection. The ring current responds by beginning to close on the dayside by trapping higher energy protons, as shown in Figure 4.30e.

Simulated differential fluxes at geosynchronous orbit are presented in Figure 4.31, which are resolved by energy and MLT. The relatively low fluxes produced by the $T_{E N A}$ boundary conditions are again apparent at geosynchronous orbit, much as they were found to be in the preceding discussion of Figures 4.29-4.30. At 0730 UT the large temperature variations in MLT from the $T_{E N A}$ model produce a rather complex picture at geosynchronous orbit (Figure 4.31a). In particular, significant low-energy populations $(2-5 \mathrm{keV})$ are found in the premidnight sector and at dawn, and 10-30 keV fluxes are depleted at midnight. In contrast, the $T_{T M}$ model produces a more dawn-dusk symmetric picture, except for a low-energy feature 
(a)

0730 UT

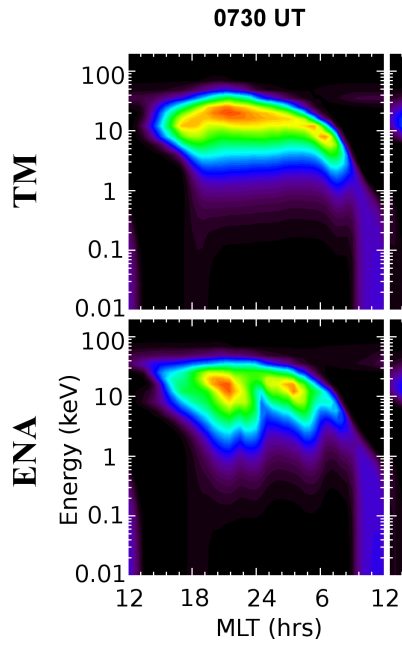

(b)

1145 UT

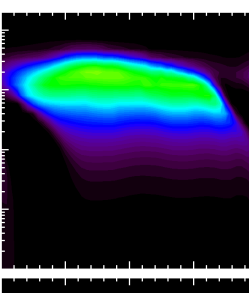

(c)

1500 UT

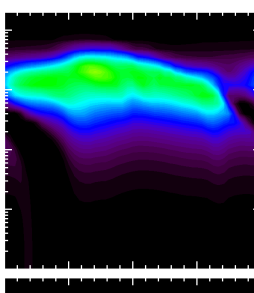

(d)

2315 UT

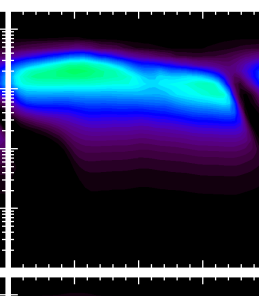

(e)

2415 UT

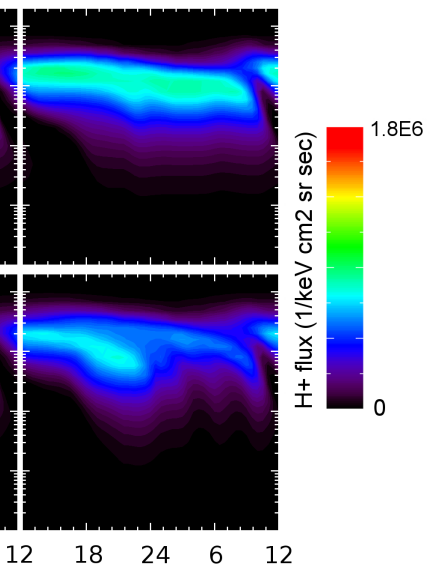

Figure 4.31: Simulated $\mathrm{H}^{+}$fluxes interpolated to geosynchronous orbit, as a function of energy and MLT, using the TM (top row) and ENA-derived (bottom row) boundary condition model. The colorbar is on a linear scale.

near dawn. The distribution of flux at geosynchronous orbit does not change significantly between the two successive intervals of southward IMF at 1145 and 1500 UT; see Figures 4.31b-c. The $T_{T M}$ model yields nearly constant flux across all MLT, whereas the $T_{E N A}$ model produces lower geosynchronous flux with more structure in MLT. Low-energy pre-midnight populations are again found from the $T_{E N A}$ simulations. Around the time interval near Sym-

$\mathrm{H}$ minimum (Figures 4.31d-e), geosynchronous fluxes from the $T_{T M}$ model decrease in overall magnitude, as compared to earlier intervals. In addition, a significant depletion of $<10 \mathrm{keV}$ flux is observed whereas the $T_{E N A}$ boundary conditions result in additional enhancements in this energy range.

\subsubsection{Data-Model Comparisons}

In this section, moments from CRCM pitch-angle averaged fluxes are taken and compared with observational data, as done earlier in Section 4.1.4 for the 22 Jul 2009 storm. Figure 
4.32 shows a data-model comparison of ion temperatures at geosynchronous orbit for all MLT at each of the 5 times discussed in the preceding section. Simulated temperatures at geosynchronous orbit are compared with geosynchronous observations extracted from TWINS ENA maps, which are overlaid in black. As before, the TWINS temperatures are calculated by integrating over the 0-32 keV energy range, and the CRCM moments are taken over the 0-25 $\mathrm{keV}$ range. Following the comparison of geosynchronous temperatures, additional comparisons are made with in situ data from THEMIS-A and THEMIS-D. THEMIS-E data is also available for this event, but is omitted from the forthcoming discussion.

As shown for the 22 July 2009 event, different boundary condition models clearly affect the spatial distribution of simulated geosynchronous temperatures, although the magnitudes at geosynchronous orbit do not strongly correlate to the prescribed boundary temperatures (see discussion of Figure 4.15 in Section 4.1.4). This is also found to be true for the 30 October 2013 event, based on the data presented in Figure 4.32.

(a)

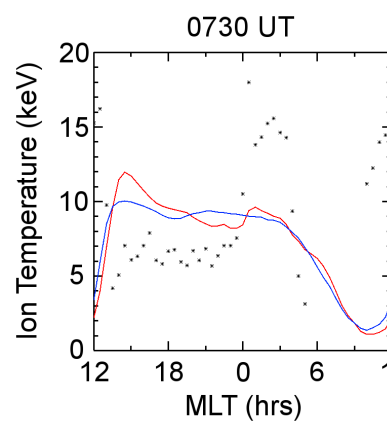

(b) 1145 UT

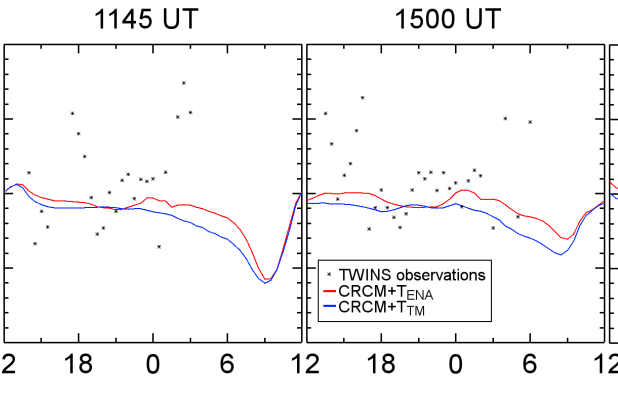

(d) 2315 UT

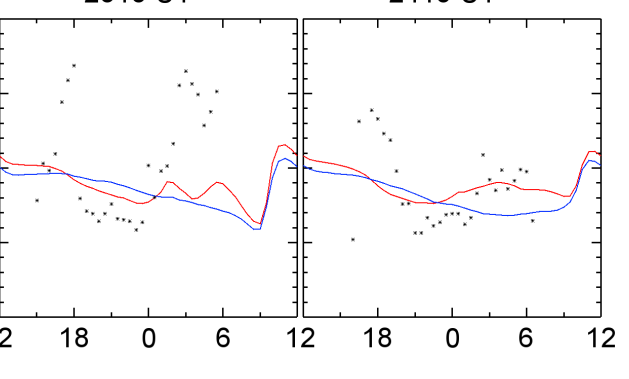

Figure 4.32: Data-model comparison of temperatures calculated at geosynchronous orbit for each boundary condition model. The red (blue) trace corresponds to the ENAderived (TM) boundary condition model. The black data represent geosynchronous temperatures obtained from ENA-derived temperature maps.

At $0730 \mathrm{UT}$ the IMF was southward at $-7 \mathrm{nT}$, marking the beginning of the event main phase. As shown in Figure 4.32a, the nearly constant (in MLT) 6-7 keV boundary conditions 
from the TM model result in geosynchronous temperatures $T_{T M, g e o}$ that are constant $(\sim 9$ $\mathrm{keV}$ ) across the entire dusk sector to about 3 MLT. $T_{E N A, g e o}$ exhibit more localized structure than $T_{T M, g e o}$ at this time, especially on the night side of the dusk-dawn meridian. This is certainly associated with the provided boundary conditions (see Figure 4.27), given the gradients present near midnight in the $T_{E N A}$ data. A $>10 \mathrm{keV}$ boundary population is supplied between 0-3 MLT, whereas temperatures at nearby MLT are 4-5 keV. A corresponding hot post-midnight population is found in the $T_{E N A, g e o}$ data alongside a cool pre-midnight depression, and the orientation of this dusk-dawn asymmetry is in agreement with the TWINS observations. This dawnward-directed temperature gradient smooths out near midnight by 1145 UT, as evidenced by the TWINS observations and the $T_{E N A}$ simulation (Figure 4.32b), although the observed pre-midnight TWINS temperatures are considerably hotter than earlier in the main phase. $T_{E N A, g e o}$ and $T_{T M, g e o}$ are both elevated at dawn as compared to earlier in this southward interval; $T_{E N A, g e o}$ increases from $6.1 \mathrm{keV}$ to $8.1 \mathrm{keV}$ and $T_{T M, g e o}$ increases from 5.7 to $7.0 \mathrm{keV}$ at $6 \mathrm{MLT}$, while both models produce roughly constant $9 \mathrm{keV}$ temperatures at 18 MLT. In the later, brief southward interval at 1500 UT (Figure 4.32c), the models and observations illustrate geosynchronous temperature distributions very similar to the earlier southward interval (Figure 4.32b). The simulated temperatures are again found to agree with TWINS observations, most importantly on the nightside. $T_{E N A, g e o}$ do show a warm post-midnight enhancement not seen in the $T_{T M, g e o}$ results or the TWINS observations. The TWINS FOV is sparse around $L=6.6$ at dawn from $1145-1500$ UT, so the significant midnight-dawn quadrant difference between $T_{E N A, g e o}$ and $T_{T M, g e o}$ cannot be directly compared with TWINS-derived temperatures. Later at Sym-H minimum, a dawnward-directed temperature gradient is observed in the TWINS data at geosynchronous orbit (Figure 4.32d) and throughout the inner magnetosphere (Figure 4.26). The orientation of the asymmetry is 
consistent with the asymmetry found in the early main phase (Figure 4.32a). The influence of the $T_{E N A}$ boundary conditions is apparent at $2315 \mathrm{UT}$, where two localized hot regions are found in $T_{E N A, g e o}$ in the midnight-dawn quadrant. $T_{T M, g e o}$ show an oppositely directed dusk-dawn temperature asymmetry, with a slightly cooler population at dawn than at dusk. One hour later, at $2415 \mathrm{UT}, T_{T M, g e o}$ again show a temperature asymmetry that is oppositely directed from TWINS observations and $T_{E N A, g e o}$ (Figure 4.32), with cooler post-midnight geosynchronous temperatures observed than at 2315 UT. The hot post-midnight patches found in $T_{E N A, g e o}$ at $2315 \mathrm{UT}$ smooth out in that region by $2415 \mathrm{UT}$, and the distribution of $T_{E N A, g e o}$ from 18 MLT to 6 MLT agree exceptionally well.

It is clear from Figures 4.32a-e that simulated proton temperatures at geosynchronous orbit agree much better with TWINS observations when using TWINS-derived boundary conditions. The lack of plasma sheet dynamics in the TM model for this particular event results cannot reproduce the localized, dynamic populations observed at geosynchronous orbit. In addition, the persistent dusk-dawn temperature asymmetry visible in the TWINS observations is only reproduced when using event-specific, spatiotemporally-resolved boundary conditions. In the following discussion, additional data-model comparisons are made between results from CRCM simulations and in situ THEMIS data.
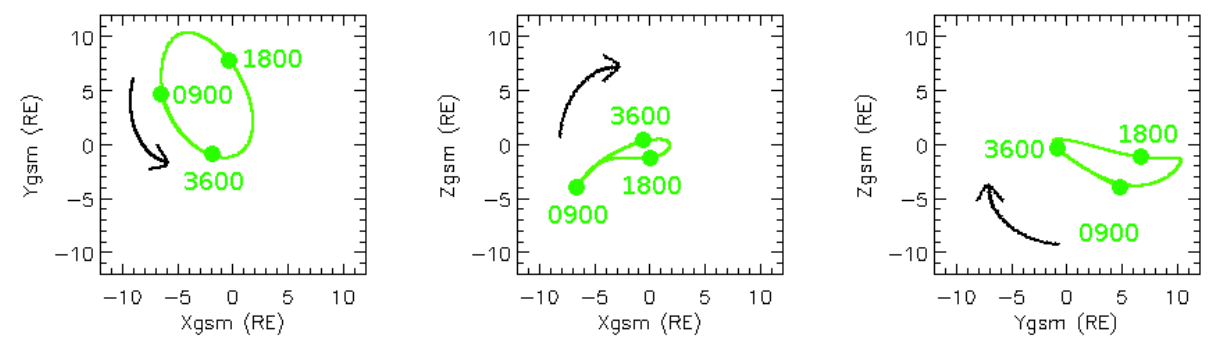

Figure 4.33: THEMIS-A orbit plots (x-y, x-z, y-z) in GSM coordinates for 0900 UT on 30 Oct 2013 to 1200 UT on 31 Oct 2013. 
THEMIS-A and THEMIS-D (TH-A, TH-D) traveled through the inner magnetosphere in similar orbits during this extended event. Here, data from TH-A and TH-D are presented and compared with results from CRCM simulations and with TWINS observations along the THEMIS spacecraft trajectories. The TH-A portion of the following analysis covers the time interval 0900 UT on 30 Oct through 1200 UT on 31 Oct. The TH-D discussion covers 1000 UT on 30 Oct to 0600 UT on 31 Oct. The two spacecraft intermittently crossed the dusk flank magnetopause outside of these time intervals. Figures 4.33 and 4.34 show the TH-A and TH-D orbits, respectively, covering the intervals mentioned; from left to right in each Figure, the GSM x-y, x-z, and y-z projections are shown.
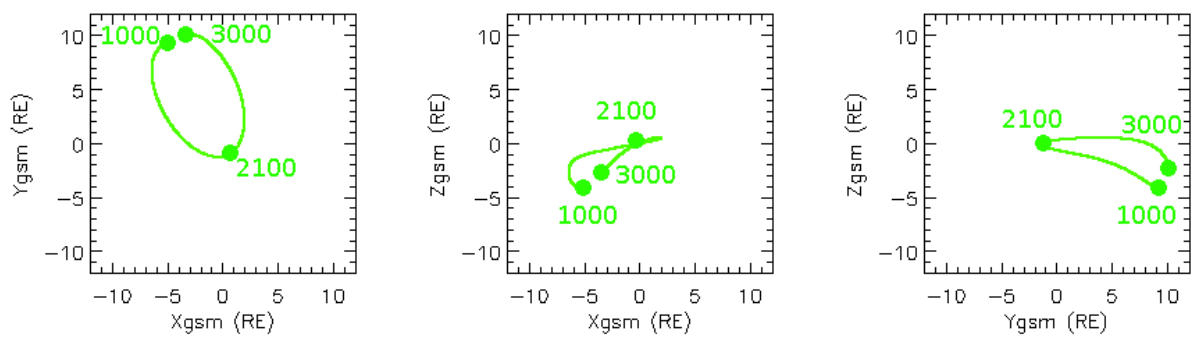

Figure 4.34: THEMIS-D orbit plots ( $\mathrm{x}-\mathrm{y}, \mathrm{x}-\mathrm{z}, \mathrm{y}-\mathrm{z})$ in GSM coordinates for $1000 \mathrm{UT}$ on 30 Oct 2013 to 0600 UT on 31 Oct 2013.

As shown, both spacecraft have apogee near $11.5 R_{E}$ and 20 MLT. TH-A and -D proceed from apogee towards the midnight meridian, making inbound crossings through geosynchronous orbit near 22.2 MLT. The spacecraft then pass through the nightside inner ring current, see perigee near 3 MLT, then proceed along their outbound pass through the postnoon ring current, crossing geosynchronous orbit at 17.5 MLT.

Figures 4.35 and 4.36 show THEMIS energy flux spectrograms (top panel), spectrograms generated from CRCM simulations (middle panels), and comparisons of proton temperature moments taken along the THEMIS trajectories (bottom). These two figures are styled the 
same as in Figures 4.17 and 4.20; the reader is directed to Section 4.1.4 for specifics of the temperature calculations. Here the observations and simulation results presented in Figures 4.35 and 4.36 are discussed in a parallel fashion.

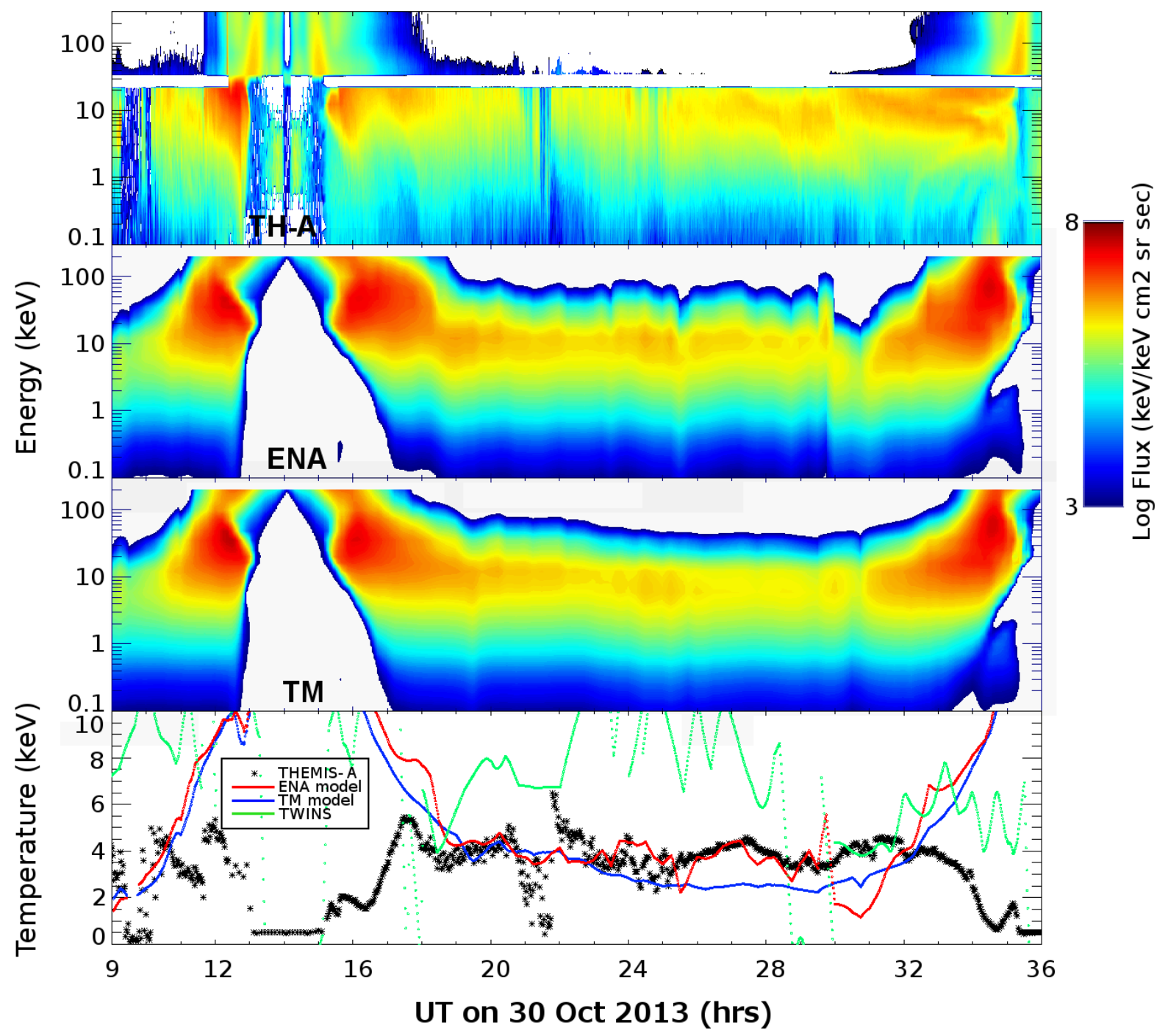

Figure 4.35: Comparison of observed and simulated THEMIS-A spectrograms from 0900 UT on 30 Oct 2013 to 1200 UT on 31 Oct 2013. Top: Observed TH-A energy spectrogram. Middle: Simulated TH-A spectrograms using the TM and ENA boundary condition models. Bottom: Ion temperatures as observed by TH-A (black) and TWINS (green) compared with CRCM results using the TM (blue) and ENA (red) boundary condition model. 


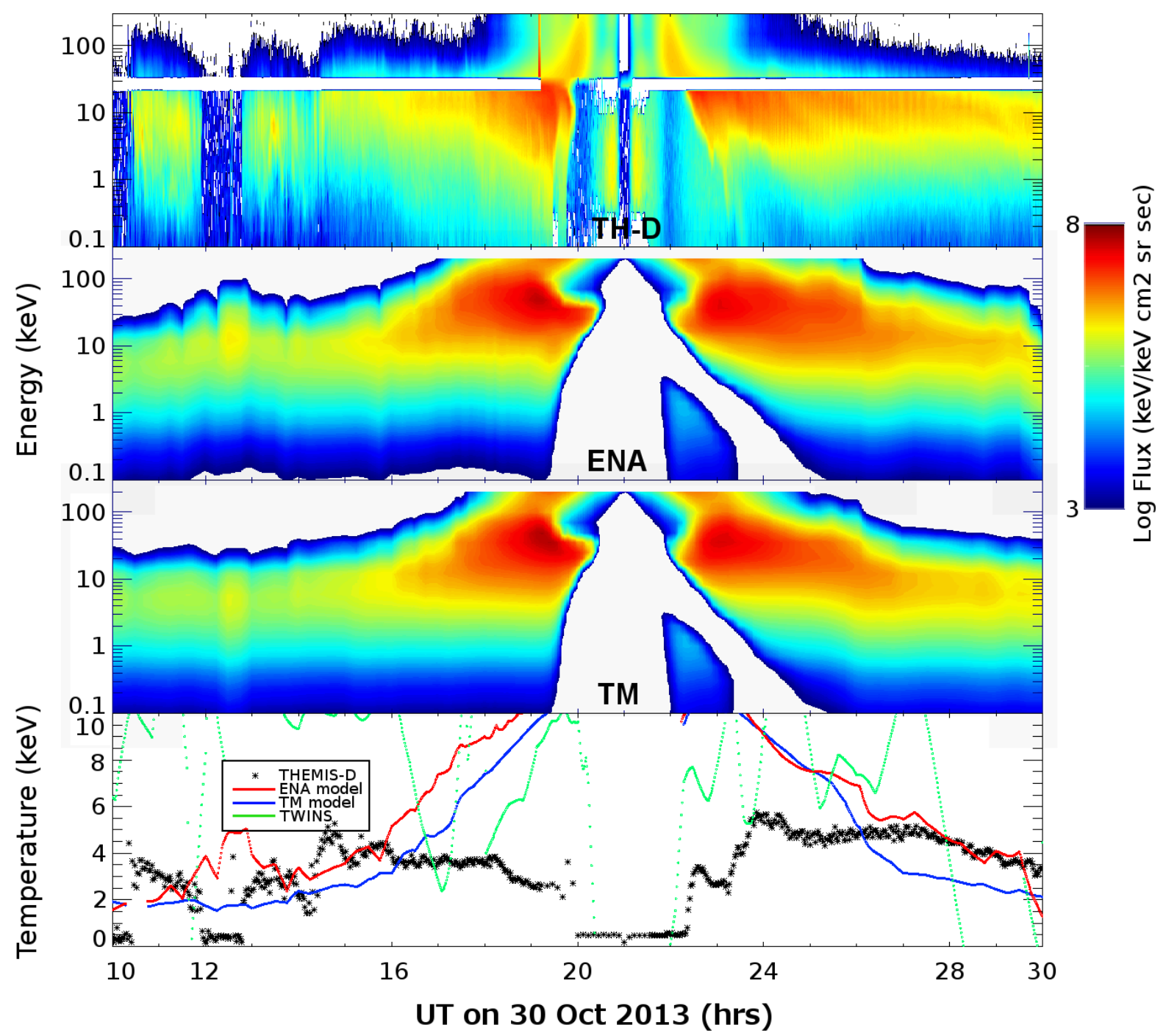

Figure 4.36: Comparison of observed and simulated THEMIS-D spectrograms from 1000 UT on 30 Oct 2013 to 0600 UT on 31 Oct 2013. Top: Observed TH-D energy spectrogram. Middle: Simulated TH-D spectrograms using the TM and ENA boundary condition models. Bottom: Ion temperatures as observed by TH-D (black) and TWINS (green) compared with CRCM results using the TM (blue) and ENA (red) boundary condition model.

TH-A is headed inbound at $0900 \mathrm{UT}$, approaching the ring current core from the premidnight sector. The TH-A spectrogram (Figure 4.35, top) shows a weak outer ring current, beyond $\sim 6 R_{E}$, wherein the fluxes produced by both CRCM simulations over-estimate the 
observed energy flux. TH-A is at geosynchronous orbit (22.5 MLT) at $1120 \mathrm{UT}$, at which time the two CRCM simulations produce similar energy spectra (middle panels) and ion temperature moments (bottom panel). The TWINS moments are a factor of 2-3 higher than those given in situ by TH-A during this inbound pass, but the fluctuations in the TWINS data are in rough agreement with how the TH-A moments fluctuate in time. TH-D was beyond $11 R_{E}$ and near 21 MLT at this time, where it sampled relatively cold $\sim 3 \mathrm{keV}$ ions (see Figure 4.36 , bottom). The hot $T_{E N A}$ boundary conditions result in high energy $(>20$ $\mathrm{keV}$ ) structure in the energy-time spectrogram which is not found in the $T_{T M}$ run. A sharp flux enhancement in the TH-A spectrogram occurs at 1140 UT, indicating a very well-defined outer ring current edge at $\sim 6 R_{E}, 21.4$ MLT. Both CRCM runs show pronounced trapped high-energy $(>25 \mathrm{keV})$ populations that aren't visible from the TH-A SST observations during this initial inbound pass. TH-A continues through the near-midnight ring current towards perigee while TH-D is near apogee. At 1150 UT, the TH-D spectrogram (Figure 4.36) shows a dropout of ion flux at all energies, corresponding in time to the northward turning of the IMF. This feature is sustained in the observed spectrogram for 45 minutes, but not reproduced well in the simulated spectrograms. The northward turning resulted in a compression of the magnetosphere, drawing the dusk-side magnetopause Earthward and thus across the TH-D spacecraft. Therefore TH-D was in the magnetosheath during this 45 minute interval. The modeled magnetopause boundary from the Shue et al. ${ }^{37}$ model, which is used as an outer boundary by CRCM, clearly over-estimates the dusk-side magnetopause standoff distance at this time, explaining the lack of a significant dropout.

As TH-A continues past the inner ring current edge through perigee, TH-D re-enters the magnetosphere at 1245 UT and continues on its inbound trajectory through the premidnight sector. The $T_{E N A}$ model produces higher magnetospheric temperatures than the 
$T_{T M}$ model during the entire TH-D inbound pass, shown in the bottom of Figure 4.36. The TWINS moments also provide considerably higher temperatures during this TH-D inbound leg, intermittently exceeding $10 \mathrm{keV}$ through the pre-midnight sector. In the outer $L$-shells where there are no trapped populations (1245-1530 UT), the computed $T_{T M}$ moments show considerably cooler temperatures than those observed by TH-D, whereas the $T_{E N A}$ moments agree more closely with TH-D temperatures and fluctuations during this interval. Comparing TH-D and simulated moments at 1330 UT and 1530 UT, one may easily infer the general trend of ion heating from $\sim 3 \mathrm{keV}$ to $\sim 4 \mathrm{keV}$. This heating is a consequence of the conservation of the first adiabatic invariant; as the spacecraft travels to lower $L$-shells, the dipolar field increases in strength thus increasing the mean energy of the ions (see Equation 2.2). TH-D reaches the plasmapause at $1600 \mathrm{UT}$, evidenced by the increase in low-energy flux in the TH-D spectrogram (Figure 4.36, top). Here, as with the 22 July 2009 event, the lack of a plasmasphere model causes the simulated temperature moments to diverge. Collisions cannot be neglected in this region, because such collisions break the adiabatic invariance and thus strongly mediate the average plasma parameters in this region.

TH-A entered the post-noon inner ring current at 1515 UT (16.1 MLT) and crossed geosynchronous orbit at $1700 \mathrm{UT}$ (17.7 MLT). TH-D was concurrently entering the core of the ring current in the pre-midnight sector. The post-noon ring current is observed by TH-A to be less intense than predicted by the two CRCM simulations. In addition, both boundary condition models produce larger high-energy $(>25 \mathrm{keV})$ fluxes than was observed in situ by TH-A. The $T_{E N A}$ model produces an abrupt depletion of high-energy flux at 1810 UT, which is a result of the global decrease in temperature boundary conditions (see Figure 4.27). On the night side, TH-D was simultaneously observing a ring current that had intensified since TH-A had observed it earlier. TH-D crossed geosynchronous orbit (22.0 MLT) at 1810 UT 
and passed into the ring current core just after 1900 UT. Here the CRCM simulations overestimate the peak flux, including in the higher energy bins. In the innermost ring current the simulations produce pronounced nose structures, indicating deeper penetration of 25-35 $\mathrm{keV}$ ions than is observed by TH-D (see Buzulukova et al. ${ }^{30}$ and references therein). TH-D then continues towards perigee as TH-A heads towards apogee across the dusk meridian. After crossing geosynchronous orbit, TH-A observes ion temperatures that are in excellent agreement with the $T_{E N A}$ moments. The local heating observed by TH-A from $1830 \mathrm{UT}$ to 2020 UT is more consistent with the $T_{E N A}$ simulated moments; TH-A temperatures increase $30 \%$ from $3.5 \mathrm{keV}$ to $4.5 \mathrm{keV}$, while the $T_{E N A}$ model temperatures increase $12 \%$ from 4.3 $\mathrm{keV}$ to $4.8 \mathrm{keV}$, and the $T_{T M}$ model temperatures increase $25 \%$ from $3.6 \mathrm{keV}$ to $4.5 \mathrm{keV}$. The observed TWINS temperatures are also increasing over this interval, but show a substantial increase up to $8 \mathrm{keV}$; there is somewhat low confidence in these temperatures, as discussed in Section 4.2.2. Sporadic flux dropouts appear in the TH-A data from 2050 UT to 2140 UT which are not reproduced by CRCM, which directly follows the strong, abrupt southward IMF turning. The TH-A spacecraft is near apogee at this time; there is an accompanying dropout in electron ESA and SST data, and a moderate rotation of the local magnetic field (not shown), which suggest a brief magnetopause crossing. As before, the Shue et al. model over-estimates the dusk flank standoff distance; otherwise, CRCM flux would not be available in this brief interval. TWINS moments show a constant $6.7 \mathrm{keV}$ population at this location; this agrees with the two simulations in that no local fluctuations are observed, although the TWINS temperatures are nearly a factor of two higher than the corresponding TH-A observations and CRCM simulations.

TH-D is crossing the post-noon inner ring current edge near the end of the main phase (2200 UT), while TH-A is near apogee. During this final segment of the main phase, TH-A 
and the two CRCM simulations all show pre-midnight temperatures of $3.6 \mathrm{keV}$ (2300 UT) at high $L$-shells; TWINS temperatures show strong fluctuations and are often found to exceed $10 \mathrm{keV}$ during this interval. Meanwhile the post-noon ring current being observed by TH-D shows no sign of the nose type ISG in the spectrogram, although the dispersionless drift hole is still observed in outbound observations and simulations; as with the inbound TH-D pass, the intense nose structure found in this dayside region indicates that trapped inner ring current populations are not well represented by CRCM for this relatively weak event. Minimum Sym-H occurs later at 2315 UT, by which time the simulated $T_{T M}$ temperature moments show considerable cooling along the TH-A trajectory. Contrast this prediction with observed temperature moments and simulated $T_{E N A}$ moments, which show slight overall heating through the early recovery phase along with additional localized fluctuations not captured in the $T_{T M}$ run. At $2400 \mathrm{UT}$, TH-D again crosses geosynchronous orbit (17.5 MLT) and proceeds towards apogee through the pre-midnight quadrant during the extended recovery phase. As with the simultaneous TH-A observations, the $T_{T M}$ model produces significantly cooler simulated TH-D temperatures during this time. TH-D continues across the dusk meridian and later through the dusk-midnight quadrant; the $T_{E N A}$ and TH-D spectrograms and temperature moments are in excellent agreement over this interval, through 3000 UT. An abrupt shift in the energy spectra is observed by TH-A at 3000 UT, which is at $10 R_{E}, 21$ MLT. This shift towards higher temperature populations is probably caused by the rapid change in the IMF $B_{z}$ component. This abrupt change in IMF parameters correlates with a rapid cooling of ion temperature boundary conditions in the $T_{E N A}$ model. That simulation responds by producing a significantly cooler local temperature than is found in the TH-A observations; in the $T_{E N A}$ spectrogram, the peak flux shifts to lower energy at this time, opposite of the shift observed by the TH-A spacecraft. As TH-A continues to 
sample the inner ring current during the late recovery phase, it is evident that the simulated ring current loss mechanisms do not decrease ring current flux rapidly enough to account for observations during this late storm phase. The high ring current flux produced by the two CRCM simulations are unrealistic, as the observed ring current flux had decreased significantly by this time.

\subsection{Event Study Comparison}

A comparative discussion of the HSS-driven event (Section 4.1) and the CME-driven event (Section 4.2) presented in this chapter is in order. Upon comparison of the TWINS-derived, time resolved 2-D ion temperature maps for each event (Figures 4.2-4.7 for the HSS event, and Figures 4.22-4.26 for the CME event), it is apparent that considerably higher magnetospheric temperatures are observed for the CME-driven event. A surprising, related feature emerges in the temperature maps for the CME-driven 30-31 Oct 2013 event: global ion temperatures remain elevated for most of the main phase, although cooler temperatures are found beginning a few hours before the Sym-H minimum. This is also the case for the HSSdriven 22 Jul 2009 event, but to a lesser extent. Keesee et al. ${ }^{33}$ also reported this result in their superposed epoch analyses of ion temperatures during CME-driven and HSS-driven events. Specifically, average ion temperatures during CME-driven events are generally much higher just after onset than near Sym-H minimum, whereas no considerable amount of such cooling was observed in their set of HSS-driven events. Global ion temperatures also in-

crease during the recovery phase of the HSS-driven event, which is less pronounced in the CME-driven event. Again, this result is consistent with the outcome of the Keesee et al. study. Figure 4.37 is taken from that publication; average ion temperatures within the range 
$-20 R_{E}<X_{\mathrm{GSM}}, Y_{\mathrm{GSM}}<20 R_{E}$ are displayed for a superposed epoch analysis of TWINS measurements for 21 CME-driven storms (left) and 15 HSS-driven storms (right).
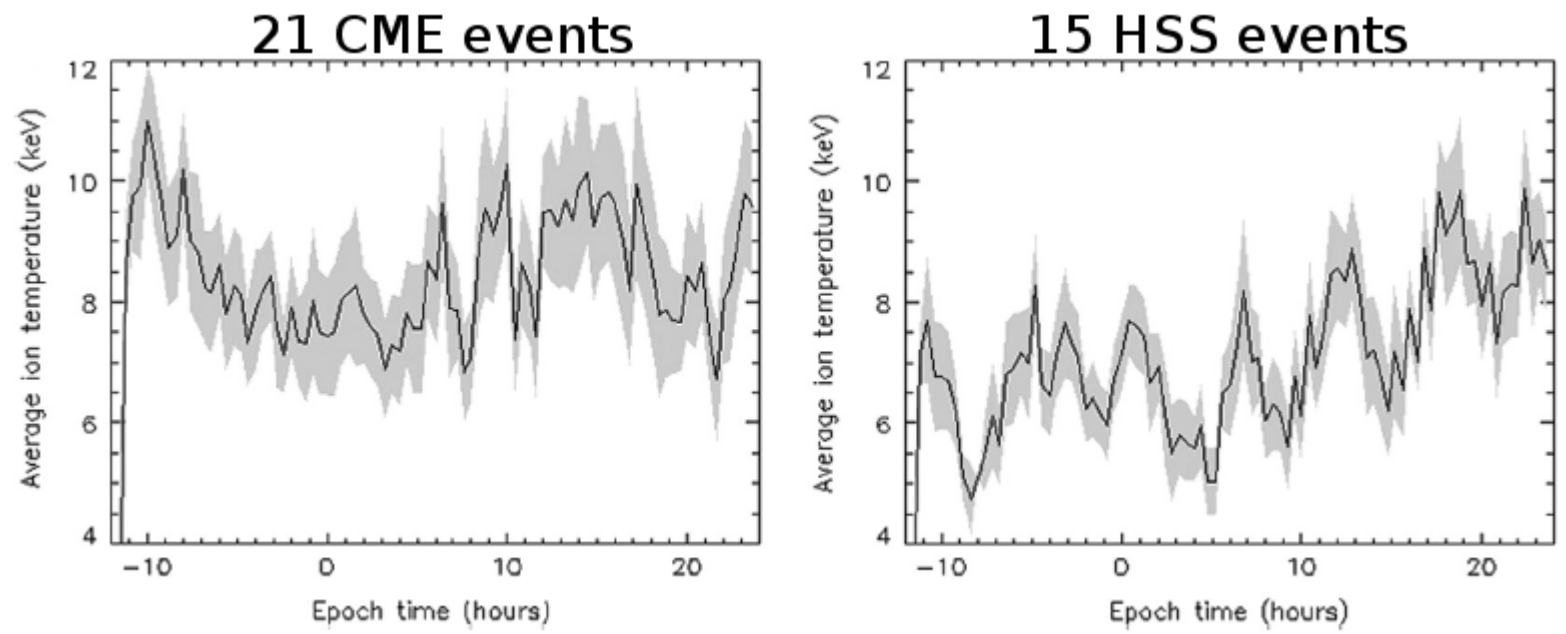

Figure 4.37: Mean ion temperature versus epoch time for 21 CME storms (left) and 15 HSS storms (right). The shaded area represents the mean temperature combined with the calculated error. Figure taken from Keesee et al.; see text.

Dayside geosynchronous temperatures near noon are abruptly elevated in the CME-driven runs near Sym-H minimum (see Figure 4.32d, e), a feature consistent with the superposed epoch analysis of Denton et al., ${ }^{34}$ whereas such localized heating is not found for the HSSdriven runs (see Figures $4.15 \mathrm{a}, \mathrm{b})$. A more complete comparison of the simulated geosynchronous temperatures is presented in Figure 4.38 alongside the geosynchronous hot proton data originally presented in the Denton et al. study. In that figure, the vertical axes correspond to the epoch time in days; zero epoch is the time of minimum Dst, marked by black dotted lines. Local time is shown on the horizontal axes. The simulations did not cover the full 3.5 day time frame, thus the absence of data in the middle and bottom rows. 

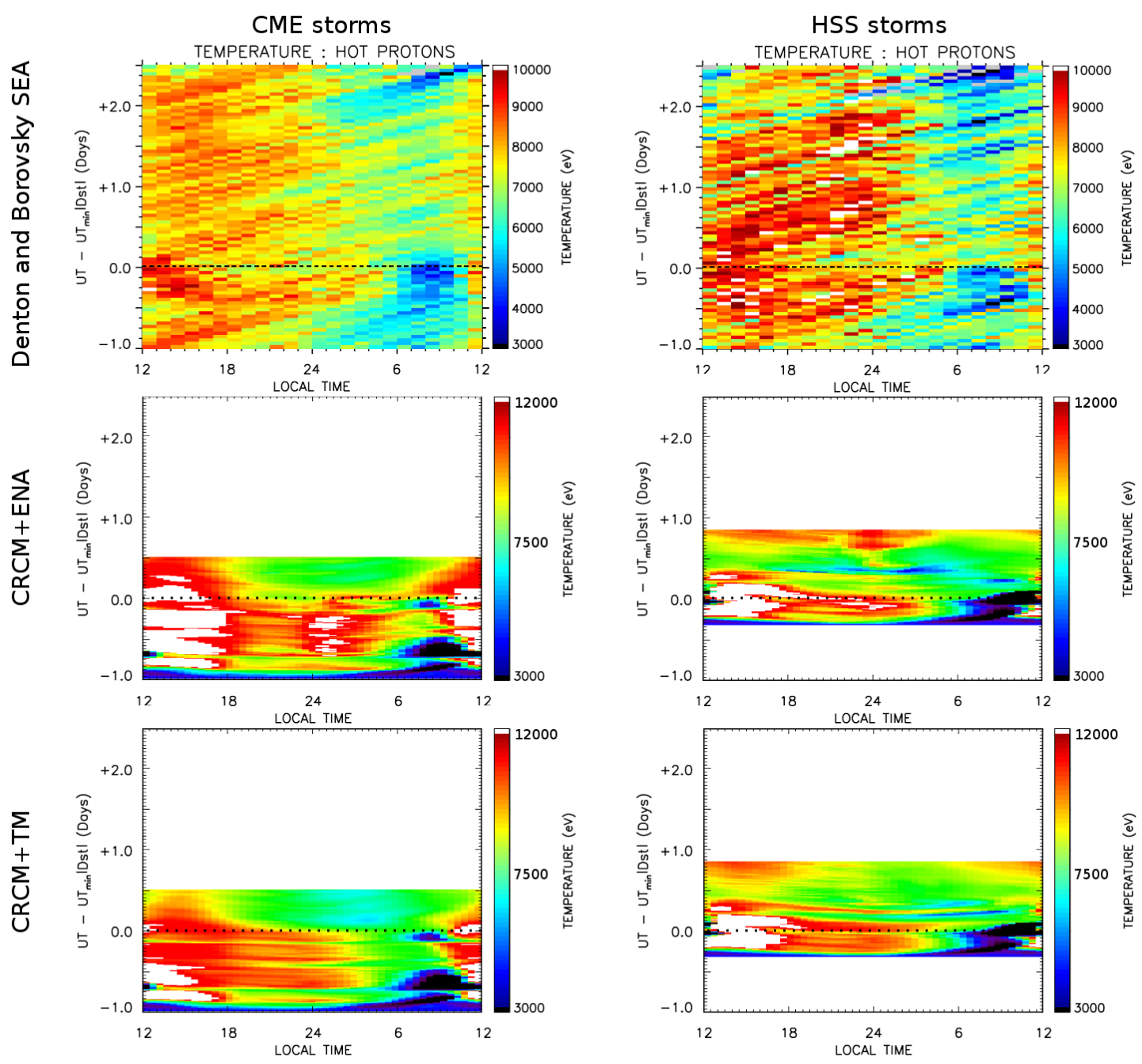

Figure 4.38: Comparison of ion temperatures from the Denton et al. superposed epoch analysis (top row) of CME- and HSS-driven events with CRCM simulations using the two boundary condition models in CRCM.

For the CME-driven events (left column), both boundary condition models produce the highest temperatures in the post-noon sector and the coolest temperatures in the dawnnoon quadrant preceding zero epoch, both agreeing with the observed features reported in the Denton et al. study (top row, left). The $T_{E N A}$ boundary condition model produces hot, $\sim 10 \mathrm{keV}$ post-midnight populations prior to zero epoch which are not present in the 
observations. The simulations also predict rapid nightside cooling immediately following zero epoch. Overall, the simulations predict higher average temperatures for the CME-driven event than for the HSS-driven event, but also reproduce important features present in the observations of HSS events. As with the CME storm, the highest simulated temperatures preceding zero epoch are found from noon across the dusk meridian to midnight. In addition, the nightside temperatures decrease after zero epoch, but this decrease is not as pronounced as in the CME simulations. The $T_{E N A}$ boundary condition model also shows significant recovery phase heating near midnight, which is in better agreement with the geosynchronous observations than the $T_{T M}$ simulation of this HSS-driven event. 


\section{References}

[1] The OMNI data were obtained from the GSFC/SPDF OMNIWeb interface at http://omniweb.gsfc.nasa.gov.

[2] B. T. Tsurutani, R. L. McPherron, W. D. Gonzalez, G. Lu, N. Gopalswamy, and F. L. Guarnieri, Magnetic Storms Caused by Corotating Solar Wind Streams, vol. 167 of Geophysical Monographs, ch. 1, pp. 1-17. American Geophysical Union, 2006.

[3] J. W. Gjerloev, "The supermag data processing technique," Journal of Geophysical Research, vol. 117, p. A09213, 2012.

[4] For the ground magnetometer data we gratefully acknowledge: Intermagnet; USGS, Jeffrey J. Love; CARISMA, PI Ian Mann; CANMOS; The S-RAMP Database, PI K. Yumoto and Dr. K. Shiokawa; The SPIDR database; AARI, PI Oleg Troshichev; The MACCS program, PI M. Engebretson, Geomagnetism Unit of the Geological Survey of Canada; GIMA; MEASURE, UCLA IGPP and Florida Institute of Technology; SAMBA, PI Eftyhia Zesta; 210 Chain, PI K. Yumoto; SAMNET, PI Farideh Honary; The institutes who maintain the IMAGE magnetometer array, PI Eija Tanskanen; PENGUIN; AUTUMN, PI Martin Conners; DTU Space, PI Dr. Jurgen Matzka; South Pole and McMurdo Magnetometer, PI's Louis J. Lanzarotti and Alan T. Weatherwax; ICESTAR; RAPIDMAG; PENGUIn; British Artarctic Survey; McMac, PI Dr. Peter Chi; BGS, PI Dr. Susan Macmillan; Pushkov Institute of Terrestrial Magnetism, Ionosphere and Radio Wave Propagation (IZMIRAN); GFZ, PI Dr. Monika Korte; SuperMAG, PI Jesper W. Gjerloev.

[5] N. A. Tsyganenko and D. P. Stern, "Modeling the global magnetic field of the large- 
scale birkeland current systems," Journal of Geophysical Research, vol. 101, no. A12, pp. 27187-27198, 1996.

[6] M. C. Fok, N. Buzulukova, S. H. Chen, P. W. Valek, J. Goldstein, and D. J. McComas, "Simulation and twins observations of the 22 july 2009 storm," Journal of Geophysical Research, vol. 115, p. A12231, 2010.

[7] P. Valek, P. C. Brandt, N. Buzulukova, M. C. Fok, J. Goldstein, D. J. McComas, J. D. Perez, E. Roelof, and R. Skoug, "Evolution of low-altitude and ring current ena emissions from a moderate magnetospheric storm: Continuous and simultaneous twins observations," Journal of Geophysical Research, vol. 115, no. A11209, pp. 1-8, 2010.

[8] P. W. Valek, J. Goldstein, D. J. McComas, R. Ilie, N. Buzulukova, M. C. Fok, and J. D. Perez, "Oxygen-hydrogen differentiated observations from twins: The 22 july 2009 storm," Journal of Geophysical Research: Space Physics, vol. 118, pp. 3377-3393, 2013.

[9] R. Ilie, R. M. Skoug, H. O. Funsten, M. W. Liemohn, J. J. Bailey, and M. Gruntman, "The impact of geocoronal density on ring current development," Journal of Atmospheric and Solar-Terrestrial Physics, vol. 99, pp. 92-103, 2012.

[10] J. D. Perez, E. W. Grimes, J. Goldstein, D. J. McComas, P. Valek, and N. Billor, "Evolution of cir storm on 22 july 2009," Journal of Geophysical Research, vol. 117, no. A09221, pp. 1-19, 2012.

[11] N. Y. Ganushkina, S. Dubyagin, M. Kubyshkina, M. Liemohn, and A. Runov, "Inner magnetosphere currents during the cir/hss storm on july 21-23, 2009," Journal of Geophysical Research, vol. 117, no. A00L04, pp. 1-18, 2012. 
[12] J. G. Elfritz, A. M. Keesee, N. Buzulukova, M. C. Fok, and E. E. Scime, "First results using twins-derived ion temperature boundary conditions in crcm," Journal of Geophysical Research: Space Physics, vol. 119, 2014.

[13] A. M. Keesee, J. G. Elfritz, D. J. McComas, and E. E. Scime, "Inner magnetosphere convection and magnetotail structure of hot ions imaged by ena during a hss-driven storm," Journal of Geophysical Research, vol. 117, no. A00L06, pp. 1-9, 2012.

[14] M. H. Denton and J. E. Borovsky, "Superposed epoch analysis of high-speed-streamdriven effects at geosynchronous orbit: Hot plasma, cold plasma, and the solar wind," Journal of Geophysical Research, vol. 113, no. A0, p. 7216, 2008.

[15] M. H. Denton and J. E. Borovsky, "The superdense plasma sheet in the magnetosphere during high-speed-stream-driven storms: Plasma transport timescales," Journal of Atmospheric and Solar-Terrestrial Physics, vol. 71, pp. 1045-1058, 2009.

[16] D. J. Southwood, "The role of hot plasma in magnetospheric convection," Journal of Geophysical Research, vol. 82, no. 7A0755, pp. 5512-5520, 1977.

[17] S. R. Elkington, D. N. Baker, and M. Wiltberger, The Inner Magnetosphere: Physics and Modeling, vol. 155 of Geophysical Monographs, ch. Injection of energetic ions during the 31 March 0630 substorm, pp. 147-154. American Geophysical Union, 2005.

[18] W. Baumjohann, Y. Kamide, and R. Nakamura, "Substorms, storms, and the near-earth tail," Journal of Geomagnetism and Geoelectricity, vol. 48, p. 177, 1996.

[19] W. Baumjohann, R. Nakamura, R. Schodel, and K. Dierschke, Disturbances in Geospace: The Storm-Substorm Relationship, vol. 142 of Geophysical Monographs, ch. Substorms, 
Storms, and the Storm-Time Plasma Sheet, pp. 55-58. American Geophysical Union, 2003.

[20] R. K. Burton, R. L. McPherron, and C. T. Russell, "An empirical relationship between interplanetary conditions and dst," Journal of Geophysical Research, vol. 80, pp. 4204$4214,1975$.

[21] A. J. Dessler and E. N. Parker, "Hydromagnetic theory of magnetic storms," Journal of Geophysical Research, vol. 64, pp. 2239-2259, 1959.

[22] N. Sckopke, "A general relation between the energy of trapped particles and the disturbance field near the earth," Journal of Geophysical Research, vol. 71, p. 3125, 1966.

[23] W. Baumjohann and R. A. Treumann, Basic Space Plasma Physics. Imperial College Press, 1997.

[24] J. C. Zhang, M. W. Liemohn, M. F. Thomsen, J. U. Kozyra, M. H. Denton, and J. E. Borovsky, "A statistical comparison of hot-ion properties at geosynchronous orbit during intense and moderate geomagnetic storms at solar maximum and minimum," Journal of Geophysical Research, vol. 111, no. A07206, 2006.

[25] T. W. Garner, "Numerical experiments on the inner magnetospheric electric field," Journal of Geophysical Research, vol. 108, no. A10, p. 1373, 2003.

[26] S. Wing, J. R. Johnson, P. T. Newell, and C. I. Meng, "Dawn-dusk asymmetries, ion spectra, and sources in the northward interplanetary magnetic field plasma sheet," Journal of Geophysical Research, vol. 110, no. A08205, 2005. 
[27] M.-B. Kallenrode, Space Physics: An Introduction to Plasmas and Particles in the Heliosphere and Magnetospheres. Springer, 3rd ed., 2004.

[28] C. McIlwain, Earth's Magnetospheric Processes, ch. Plasma convection in the vicinity of the geosynchronous orbit, p. 268. D. Reidel Pub. Comp., 1972.

[29] H. Shirai, K. Maezawa, M. Fujimoto, T. Mukai, Y. Saito, and N. Kaya, "Monoenergetic ion drop-off in the inner magnetosphere," Journal of Geophysical Research, vol. 102, no. A9, pp. 19873-19881, 1997.

[30] N. Y. Buzulukova, Y. I. Galperin, R. A. Kovrazhkin, A. L. Glazunov, G. A. Vladimirova, H. Stenuit, J. A. Sauvaud, and D. C. Delcourt, "Two types of ion spectral gaps in the quiet inner magnetosphere: Interball-2 observations and modeling," Annales Geophysicae, vol. 20, pp. 349-364, 2002.

[31] A. J. Dessler, W. E. Francis, and E. N. Parker, "Geomagnetic storm suddencommencement rise times," Journal of Geophysical Research, vol. 65, no. 9, pp. 2715$2719,1960$.

[32] L. F. Burlaga and K. W. Ogilvie, "Causes of sudden commencements and sudden impulses," Journal of Geophysical Research, vol. 74, no. 11, pp. 2815-2825, 1969.

[33] A. M. Keesee, J. G. Elfritz, D. J. McComas, and E. E. Scime, "Superposed epoch analyses of ion temperatures during cme and cir/hss-driven storms," Journal of Atmospheric and Solar-Terrestrial Physics, vol. 115-116, pp. 67-78, 2013.

[34] M. H. Denton, J. E. Borovsky, R. M. Skoug, M. F. Thomsen, B. Lavraud, M. G. Henderson, R. L. McPherron, J. C. Zhang, and M. W. Liemohn, "Geomagnetic storms driven 
by icme- and cir-dominated solar wind," Journal of Geophysical Research, vol. 111, p. A07S07, 2006.

[35] Y. Ebihara and M. Ejiri, "Simulation study on fundamental properties of the stormtime ring current," Journal of Geophysical Research, vol. 105, no. A7, pp. 15843-15859, 2000.

[36] N. A. Tsyganenko and T. Mukai, "Tail plasma sheet models derived from geotail particle data," Journal of Geophysical Research, vol. 108, no. A3, p. 1136, 2003.

[37] J. H. Shue, J. K. Chao, H. C. Fu, C. T. Russell, P. Song, K. K. Khurana, and H. J. Singer, "A new functional form to study the solar wind control of the magnetopause size and shape," Journal of Geophysical Research, vol. 102, no. A5, pp. 9497-9511, 1997. 


\section{Chapter 5}

\section{Parametric Analysis of}

\section{Magnetospheric Ion Temperatures}

In this chapter, average magnetospheric ion temperatures are obtained from TWINS ENA measurements for specific ranges of solar wind parameters, IMF parameters, and geomagnetic indices. 'Instantaneous' ENA-derived temperatures (e.g., Figure 4.2) are calculated in the manner discussed in Chapter 3 and used as spatiotemporally resolved boundary conditions for event studies in Chapter 4. Average global ion temperatures are calculated using the same methodology for a 31-month interval of time corresponding to the most recent geomagnetically active period, specifically from 1 Jan 2011 through 31 Jul 2013. This time frame corresponds to the early active period of solar cycle 24 through a near-maximum in solar cycle 24. Although TWINS observations are available beginning in 2008, solar minimum lasted until late 2010 and thus that time frame is neglected in this analysis. Figure 5.1 shows the sunspot number versus time since 2000; the data contained therein is provided through the NOAA Space Weather Prediction Center. Global ion temperature maps are averaged 
over all time intervals within the full 31-month time frame that satisfy the user-defined parametric conditions. Such an analysis provides a 31-month average correlating specific solar wind parameters with the global magnetospheric response.

\section{ISES Solar Cycle Sunspot Number Progression}

Observed data through Apr 2014

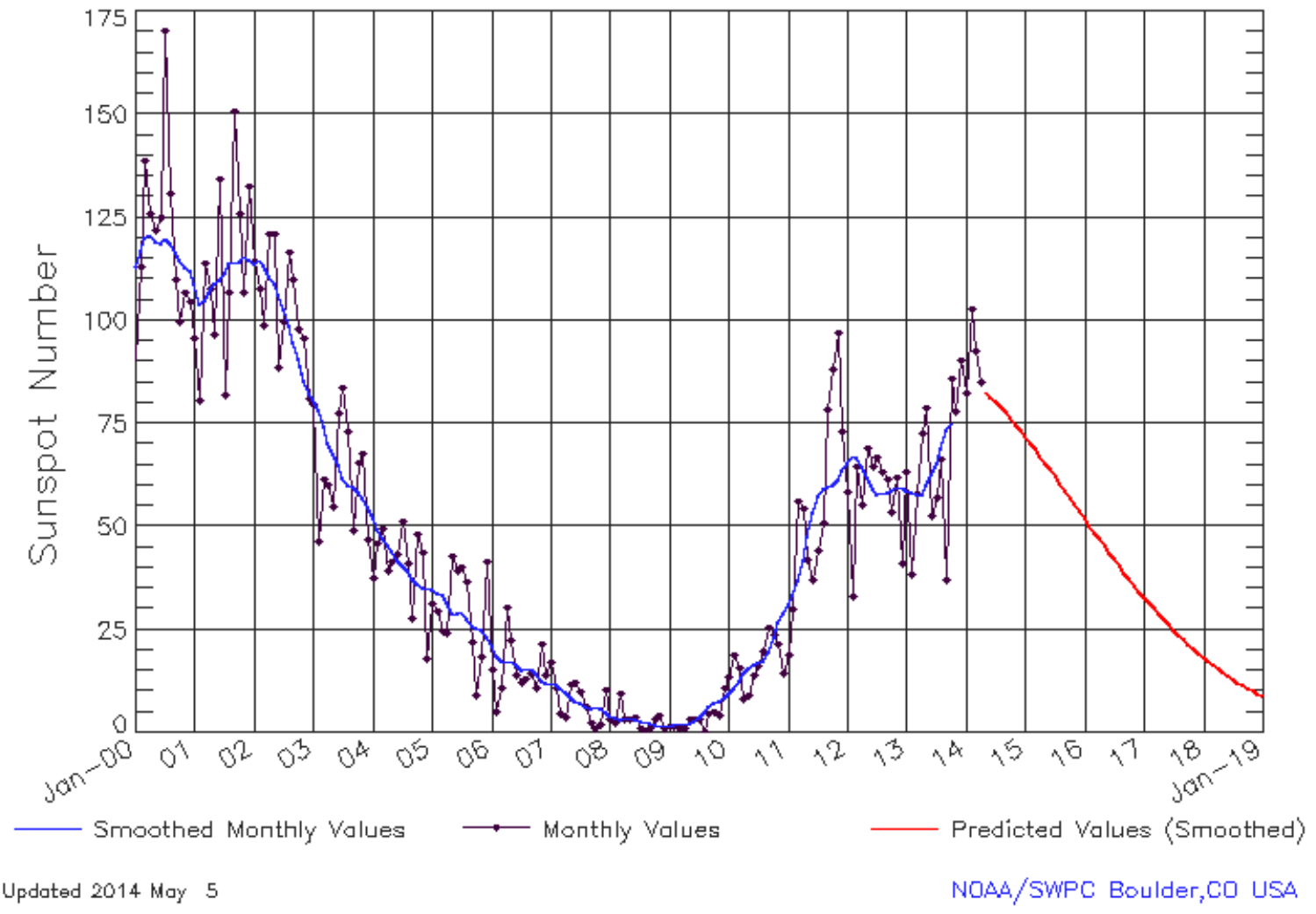

Figure 5.1: Sunspot number versus time since the year 2000, showing the end of solar cycle 23 and the period of increasing activity of solar cycle 24. Figure obtained through NOAA SPWC.

This is the first study using TWINS-based temperatures around solar maximum in this way. A complementary quiet-time study employing TWINS temperatures has been performed by Keesee et al., ${ }^{1}$ where the authors compiled 138 hours of TWINS ENA fluxes from early 2009 (solar minimum, see Figure 5.1) to show that the dusk-dawn asymmetry in ion temperature agrees with the asymmetry predicted by the finite tail width model of Spence 
and Kivelson. ${ }^{2}$ Zaniewski et $a{ }^{3}{ }^{3}$ presented a superposed epoch analysis of 39 geomagnetic storms during 2000-2002 (solar maximum) and binned MENA instrument fluxes and corresponding temperatures by storm phase, and illustrated the reliability of this statistical approach in conjunction with ENA images provided by IMAGE.

A similar analysis based on TWINS measurements is presented in this chapter, which covers a large fraction of the interval of increasing activity for solar cycle 24. This analysis offers improved temporal and spatial resolution than was possible for the Zaniewski et al. study. In addition, this is the first such statistically-averaged study using TWINS ENAderived temperatures during active periods. Initial, unpublished results of this study were presented by Scime and Keesee. ${ }^{4}$ In this chapter, the focus is on intervals with Dst<-30 nT to completely differentiate from and thus complement the Keesee et al. study.

\subsection{TWINS Binning Procedure and the ACE Data Set}

Solar wind data is provided by the ACE solar wind monitor which is located at the L1 Lagrange point; this data is provided through NASA OMNIWeb, and in all cases presented here the data is time-shifted to the bow shock nose. The 31-month period is scanned for intervals in which the solar wind, IMF, and geomagnetic parameters fall into specific bins. Specifically, ACE data is binned by IMF $B_{z}$ orientation (positive or negative) and solar wind dynamic pressure $P_{d y n}$ (high or low). High pressure is defined to be the range $4<P_{d y n}<15$ $\mathrm{nPa}$, and low pressure is defined to be where $0.1<P_{d y n}<4 \mathrm{nPa}$. The IMF components are in GSM coordinates. ACE data is also filtered by activity level by constraining the data set with the Dst geomagnetic index in order to separate magnetospheric ion temperature response during storm phases as well as separate the response for storms of different severity. Since 
ENA fluxes must be integrated in time in order to meet statistical significance requirements, the primary restricting condition is that solar wind parameters must remain within a certain bin for at least 30 instrument actuator sweeps (a little less than one hour). If the solar wind parameters remain within a specified bin for longer than 40 sweeps, the time frame is subdivided into separate intervals. In addition, a constraint on the TWINS satellite locations is applied such that the $Z_{\mathrm{GSM}}$ component of the orbit must be greater than $4 R_{E}$ to ensure that ENA fluxes measured near perigee are omitted from the data sets.

\subsection{Single-Parameter Dependences}

In this section, average 2-D ion temperature maps are presented to show dependence on single indices, solar wind parameters, and IMF parameters. Figures 5.2, 5.3, and 5.4 show simple dependence of ion temperatures on Dst index, solar wind pressure, and IMF $B_{z}$ orientation, respectively, for both TWINS 1 and TWINS 2 spacecraft. Figures 5.2-5.4 are organized as follows. TWINS 1 data are shown in the left column, TWINS 2 data are shown in the middle column, and a combined average of TWINS 1 and 2 is shown in the right column. Different ranges of the parameter of interest are separated into the rows in those figures, and temperatures are on a 0-20 keV colorbar. Each Earth-centered panel within the figures spans $\pm 20 R_{E}$ in $\mathrm{X}$ and $\mathrm{Y}$, both in GSM coordinates. Data near the magnetopause are found to be highly variable because a model magnetopause boundary (Shue et al.) is used to constrain which pixels are within the magnetosphere at each contributing 30-40 sweep time interval. Because the model magnetopause boundary depends solely on solar wind conditions and therefore moves in time, data outside of the mean magnetopause location is sparse. 


\subsubsection{Dst Dependence}

The dependence of global ion temperatures on the Dst index during active periods is presented in Figure 5.2. From top to bottom, average ion temperatures corresponding to the ranges $-30>$ Dst $>-60 \mathrm{nT}$ (weak activity), $-60>$ Dst $>-100 \mathrm{nT}$ (moderate activity), and -100 nT $>$ Dst (strong activity) are shown. In the weak activity range (top row), the TWINS 1 data set is accumulated from 32566 actuator sweeps, covering 638 hours of time in which the Dst index fell within the prescribed range. Similarly, the TWINS 2 data is based on 31512 sweeps and 619 hours in time. For moderately active intervals (middle row), the TWINS 1 map is based on 7829 sweeps (153.8 hours) and TWINS 2 data is based on 7094 sweeps (139 hours). For high-activity intervals where Dst $<-100 \mathrm{nT}$ (bottom row), TWINS 1 data is based on 1769 sweeps (34.8 hours), and TWINS 2 is based on 1415 sweeps (27.8 hours).

During periods of weak geomagnetic activity (top row), TWINS 1 and TWINS 2 measurements both produce higher dawn sector temperatures than those at dusk, although the TWINS 2 temperature map suffers from apparent mismatching between the two instrument sensor heads. The temperatures from each satellite are in good agreement inside of $10 R_{E}$ near dusk. Average ion temperatures between $6.6 R_{E}$ and $10 R_{E}$ on the dusk meridian are found to be $5.0 \mathrm{keV}$ from TWINS 1 and $6.4 \mathrm{keV}$ from TWINS 2. A warm, isolated feature is also present inside of the duskside magnetopause flank in both TWINS 1 and TWINS 2 data sets, although the spatial extent of the region is considerably larger in the TWINS 2 temperature map. In the moderate Dst range, TWINS 1 shows nightside temperatures that are elevated as compared to the weaker activity range (top row); the average midnight and plasma sheet temperature at $10 R_{E}$ observed by TWINS 1 is $7.7 \mathrm{keV}$ for this moderate level of activity; compare that with $6.5 \mathrm{keV}$ observed by TWINS 1 at midnight, $10 R_{E}$ during weaker activity. TWINS 2 observes $8.1 \mathrm{keV}$ ion temperatures at midnight, $10 R_{E}$ 


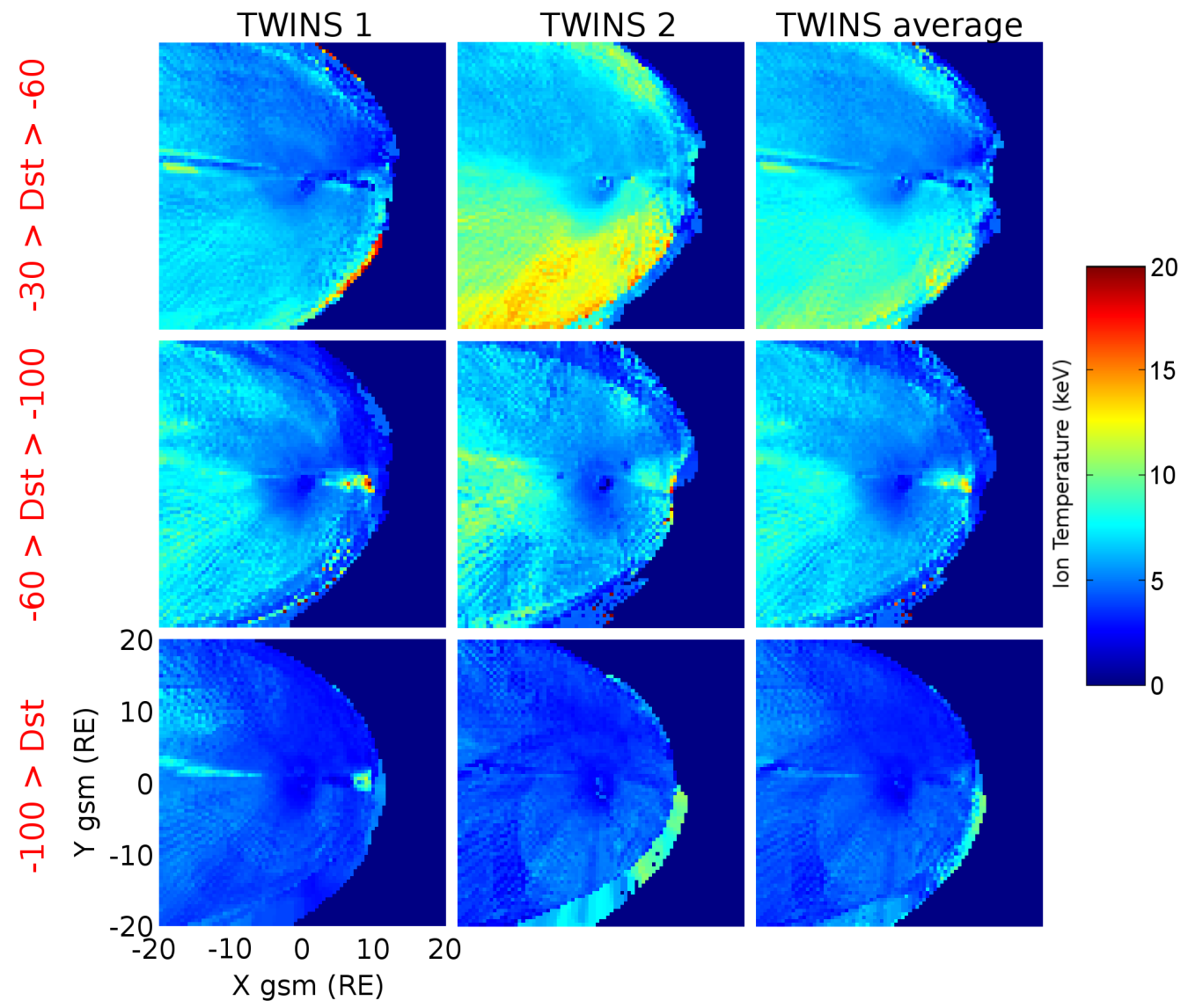

Figure 5.2: Average 2-D ion temperature maps as a function of Dst. From top to bottom, the panels show temperatures for $-60<$ Dst $<-30 \mathrm{nT},-100<$ Dst $<-60 \mathrm{nT}$, Dst $<-100 \mathrm{nT}$. The left and middle columns show temperatures from TWINS 1 and TWINS 2 data, respectively. The right column shows combined, TWINS $1+2$ average temperatures.

for moderate activity. This is slightly higher than found with TWINS 1, but the spatial distribution of ion temperatures as observed by TWINS 1 and 2 are in excellent agreement with each other. Both satellite averages produce a more dawn-dusk symmetric distribution than for the weaker activity range. Patches of hot, $\sim 12 \mathrm{keV}$ ions are observed by TWINS 2 beyond $10 R_{E}$ downtail. TWINS 1 and 2 also both show highly localized, 10-20 keV populations at the dayside magnetopause nose. It is probable that dayside reconnection associated with periods of sustained southward IMF $B_{z}$ is responsible for this heating, since 
such an IMF orientation is required to drive the Dst index into this range. One overarching, immediately apparent feature is that more disturbed conditions do not necessarily correlate one-to-one with higher inner magnetospheric ion temperatures. Both TWINS 1 and 2 show relatively cool, homogeneous $4-5 \mathrm{keV}$ temperatures throughout the magnetosphere for periods of Dst $<-100 \mathrm{nT}$ (bottom row) as compared to periods of weak and moderate geomagnetic activity (top, middle rows). A signature of dayside reconnection is again found in the TWINS 1 data set, specifically the localized $12 \mathrm{keV}$ region near the mean location of the magnetopause nose. An explanation for the considerably cooler temperatures observed for the Dst $<-100 \mathrm{nT}$ range follows. Keesee et al. ${ }^{5}$ performed a superposed epoch analysis of 48 recent storms, covering the time frame from June 2008 to April 2012. It was reported in that study that average ion temperatures near Sym-H minimum were lower than those observed closer to storm commencement and during the early main phase when the storm was driven by a CME. All 13 storms with Dst $<-100$ nT in the 31-month time interval of interest here were driven by CMEs, and in addition the most intense event (24 Apr 2012) had a minimum Sym-H of -150 nT. The mean Sym-H of all 13 of these CME events is -126 nT. The recovery phase for CME-driven storms typically begins immediately following the Sym-H minimum, and none of these 13 events have a so-called 'double dip' in the Sym-H index (for an example, see top panel of Figure 4.1) where the Sym-H remains below -100 nT between successive minima, and thus the Dst $<-100 \mathrm{nT}$ panels in Figure 5.2 are averaged only over intervals near the zero epoch of the Keesee et al. study. Thus the results presented in this parametric analysis are consistent with the findings of Keesee et al. Therefore, based on their findings it reasonable to expect that cooler global ion temperatures would emerge in the present analysis for this Dst range although further corroboration with observations is desirable. 


\subsubsection{Solar Wind Pressure Dependence}

Here, the response of average magnetospheric ion temperatures is computed as a function of the solar wind dynamic pressure. It is well known that solar wind pressure contributes

to the coupling between the solar wind and the terrestrial magnetosphere, ${ }^{6}$ especially on the dawn-dusk asymmetry in the ring current. ${ }^{7,8}$ Here, the 31 -month data set is constrained to active periods only by selecting intervals in which the Dst index is less than $-30 \mathrm{nT}$. Figure 5.3 shows average ion temperatures during active periods for low pressure (bottom row, $0.1<P<4.0 \mathrm{nPa}$ ) and high pressure (top row, $4.0<P<15.0 \mathrm{nPa}$ ). The low pressure data set is based on an accumulation of 31050 TWINS 1 actuator sweeps (609.4 hours), and 34797 sweeps (684.0 hours) for TWINS 2. Much less high pressure data is available; TWINS 1 data is averaged over 1782 sweeps (35.0 hours), while TWINS 2 data is averaged over 1669 sweeps (32.6 hours).

As expected, high solar wind pressure compresses the magnetosphere and thus forces the mean magnetopause location Earthward. Overall, magnetospheric ions are observed by TWINS to be considerably hotter during intervals of enhanced solar wind pressure. In addition, the TWINS observations in Figure 5.3 suggest that the storm-time dawn-dusk asymmetry is more pronounced during periods of enhanced pressure; in addition, the orientation of the observed asymmetry is opposite of the typical asymmetry orientation. As mentioned above, the high pressure data set displayed here (top row) is limited and therefore has a higher associated error than for storm-time periods of low dynamic pressure. Still, TWINS 1 observed the same dawn-dusk temperature asymmetry orientation during intervals of high and low pressure alike. Under the influence of high pressure, the temperature at $8 R_{E}$ on the dusk meridian is $7.5 \mathrm{keV}$ and the dawn meridian temperature at $8 R_{E}$ is $12.6 \mathrm{keV}$, giving a dawn-to-dusk ratio of $\sim 1.7$; during low pressure intervals the average ion temperatures at 


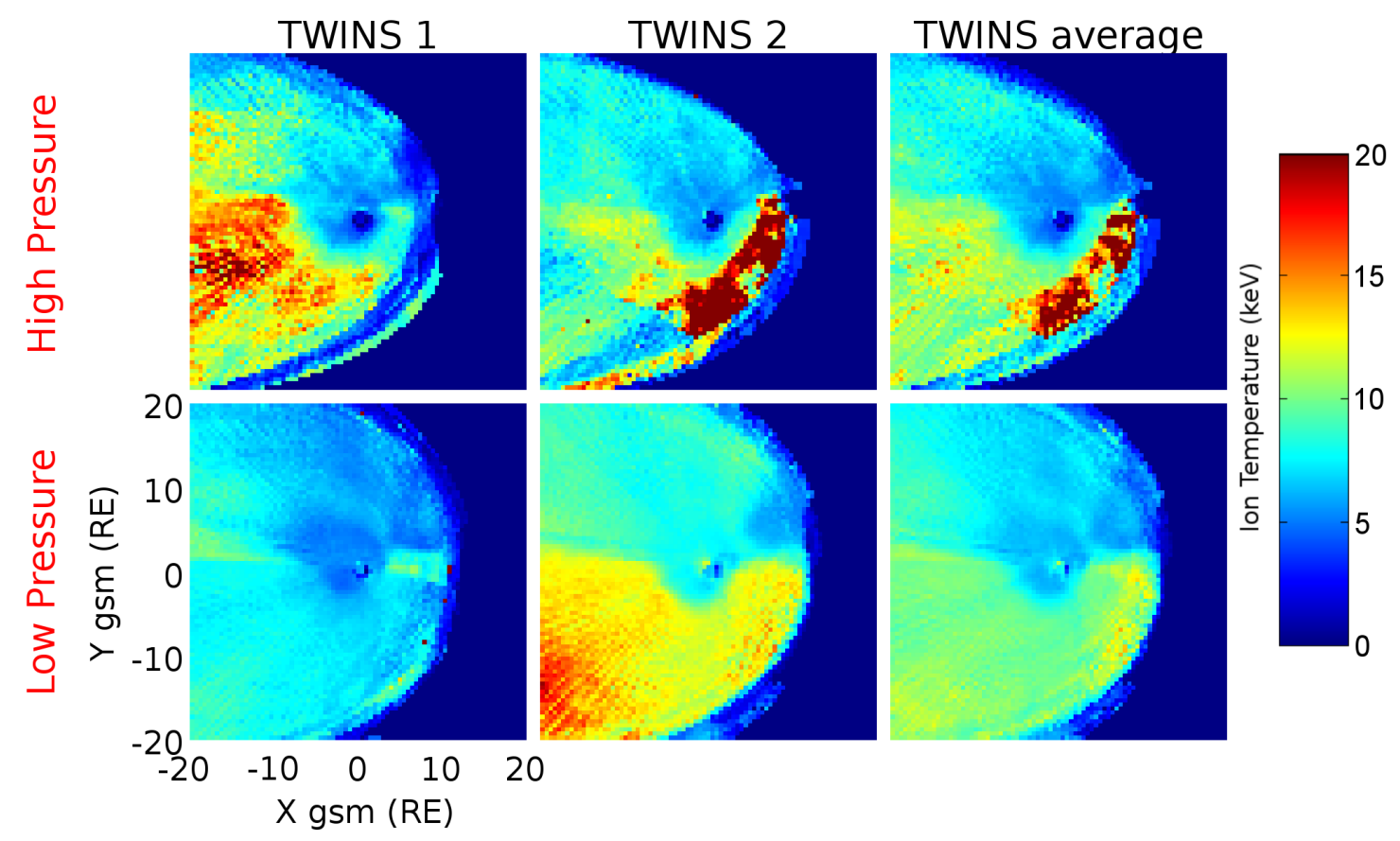

Figure 5.3: Average 2-D ion temperature maps for high (top row) and low (bottom row) solar wind pressure during intervals. The bottom and and top rows show temperatures for $0.1<P<4.0 \mathrm{nPa}$ and $4.0<P<15.0 \mathrm{nPa}$, respectively.

these locations are $6.0 \mathrm{keV}$ and $7.9 \mathrm{keV}$, respectively, giving a decreased dawn-to-dusk ratio of $\sim 1.3$ which suggests that the intensity of this asymmetry may be positively correlated with solar wind pressure enhancements. Unfortunately the data set provided by TWINS 2 does not admit the direct comparison of dawn and dusk temperatures due to the artificial asymmetry produced by poorly matched instrument heads in the low pressure case. Still, upon considering low pressure TWINS 2 observations from the entire dawn sector separately from the entire dusk sector, it is found that the average temperatures are approximately homogeneous within the separate sectors. The dusk sector temperatures beyond $\sim 10 R_{E}$ do not show a sensitive dependence on dynamic pressure; roughly $8 \mathrm{keV}$ temperatures are observed by TWINS 2 in this region regardless of the solar wind pressure. The dawn sector temperatures and the spatial distribution thereof, on the other hand, do appear to have 
strong dependence on solar wind forcing. This most apparent in the dawn-noon sector where an extended region of hot ions are found inside of the magnetopause when the dynamic pressure is high. This is presumably caused by compressional heating due to conservation of the first adiabatic invariant, although no prior observational studies exist with which such heating can be corroborated.

\subsubsection{IMF $B_{z}$ Dependence}

As discussed in Section 1.2, the orientation of the IMF $B_{z}$ component is one of the primary controlling factors on the structure and character of the storm-time plasma sheet. Here, TWINS observations of magnetospheric ion temperatures are averaged over the active periods (Dst<-30 nT) within the 31-month time period of interest and subdivided into bins where the IMF $B_{z}$ is northward $\left(0.1<B_{z}<40 \mathrm{nT}\right)$ and southward $\left(-40<B_{z}<-0.1 \mathrm{nT}\right)$. Figure 5.4 shows average ion temperatures for northward IMF $B_{z}$ (top row) and southward IMF $B_{z}$ (bottom row), as measured by TWINS 1 (left column) and TWINS 2 (middle column). The northward TWINS 1 data set is compiled from 12608 actuator sweeps, spanning 247.7 hours in time, and the southward TWINS 1 data set spans 20224 sweeps (396.7 hours). The northward and southward data sets from TWINS 2 cover 14991 sweeps (294.0 hours) and 21475 sweeps (421.4 hours), respectively.

Based on prior studies of the plasma sheet response to different IMF $B_{z}$ conditions (see discussion in Chapter 1), it is expected that southward $B_{z}$ will result in a hotter plasma sheet population than for northward $B_{z}$. The TWINS 1 data displayed in Figure 5.4 confirms this expectation. This is apparent in the post-midnight sector where TWINS 1 reports $10.5 \mathrm{keV}$ temperatures near $10 R_{E}$ under southward conditions, and $\sim 7 \mathrm{keV}$ temperatures under northward conditions. The pre-midnight sector between 21 MLT and 24 MLT does not 


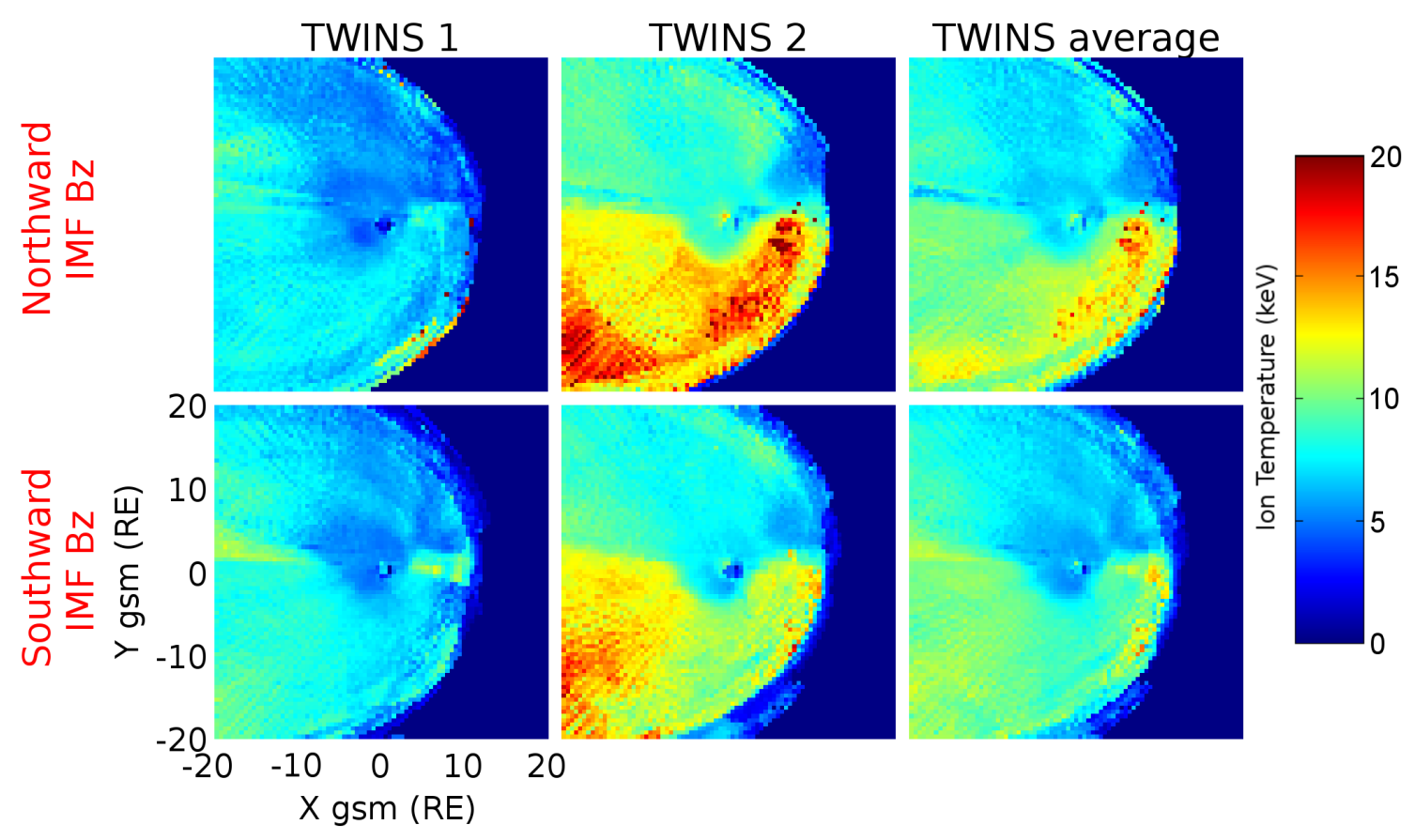

Figure 5.4: Average 2-D ion temperature maps for northward (top row) and southward (bottom row) IMF $B_{z}$ during intervals with Dst<-30 nT.

show a significant dependence on the $B_{z}$ orientation, although hotter temperatures are observed under southward conditions closer to the dusk meridian. The TWINS 1 data set also shows a high temperature enhancement localized near the magnetopause nose, again being consistent with dayside reconnection between the northward terrestrial field and the southward oriented IMF. As expected, this feature is not present in the TWINS 1 temperature map under northward $B_{z}$ conditions. As in Figures 5.2 and 5.3, the TWINS 2 temperature maps again suffer from head mismatching that is not as prominent in the TWINS 1 maps. This is apparently due to voltage differences between the two TWINS 2 instrument heads, particularly in the detection and calibration electronics packages, that are not as significant in the TWINS 1 instrument. Still, it is advantageous to separately consider each half of the magnetospheric X-Y plane imaged by TWINS 2 (dusk half and dawn half). Under 
northward IMF conditions in the dusk-midnight quadrant, TWINS 2 agrees reasonably well with TWINS 1 temperatures, although TWINS 1 shows a cooler, $\sim 5.5 \mathrm{keV}$ population centered around the dusk meridian. These somewhat subtle differences are in nearly perfect agreement with the results reported by Wing and Newell, ${ }^{9}$ where the authors presented data from low-altitude observations which suggest that inner plasma sheet ion temperatures from southward and northward intervals differ the most near 21 MLT and 3 MLT. The TWINS 2 data set shows hotter populations in the pre-noon quadrant than in the post-midnight quadrant for northward IMF, which can be attributed to adiabatic energization along particle drift paths originating from the post-midnight plasma sheet. During sustained northward intervals, the convection electric field pattern causes flux enhancements towards noon across the dawn meridian (see Figure 4.10). In addition, during intervals of northward IMF $B_{z}$ the dayside terrestrial field is compressed, and ions are thus heated through conservation of the first adiabatic invariant along their noonward drift paths. During periods of southward IMF $B_{z}$, no such dayside heating is observable in the TWINS 2 temperature map. Ion drift paths in the post-midnight sector are skewed westward under southward IMF, severely limiting ion flux noonward of the dawn meridian. Hot, westward convecting populations are lost to the dayside magnetopause before reaching the pre-noon sector, which explains the lack of hot ion populations observed in that region during intervals of southward IMF.

\subsection{Multi-Parameter Dependences}

In the same style as in Section 5.2, ion temperature maps generated by applying simultaneous constraints on input parameters are presented in this section. Since the solar wind pressure and IMF $B_{z}$ are known to correlate strongly with magnetospheric ion temperatures (see 
Section 3.2), it is desirable to present global storm-time temperatures simultaneously binned by both of these parameters for periods with Dst<-30 nT. This binning method results in four sets of data: temperatures during intervals of (1) northward IMF and high pressure, (2) southward IMF and high pressure, (3) northward IMF and low pressure, and (4) southward IMF and high pressure. The two high pressure cases are shown in Figure 5.5 and the low pressure cases are presented in Figure 5.6. In each figure, the northward case is displayed in the top row and the southward case is shown in the bottom row. The high pressure, northward IMF data are averages based on 241 sweeps from TWINS 1, covering 4.8 hours, and from 652 sweeps from TWINS 2, covering 12.8 hours. The high pressure, southward IMF temperature maps are averaged over 1541 sweeps and 30.3 hours of TWINS 1 data, and 1017 sweeps over 19.9 hours of TWINS 2 measurements. TWINS 1 temperatures during intervals low pressure and northward IMF are based on 12367 sweeps (242.9 hours), and the corresponding TWINS 2 temperatures are based on 14339 sweeps (281.3 hours). Finally, for low pressure and southward IMF, the TWINS 1 and TWINS 2 observations are averaged over 18683 sweeps (366.5 hours) and 20458 sweeps (401.5 hours), respectively.

Because of the low occurrence rate of high solar wind pressure, the statistical uncertainty associated with the ion temperatures in Figure 5.5 is relatively high. TWINS 1 measurements show signs of head mismatch at the midnight meridian, producing higher post-midnight temperatures than those on the dusk side. This result has also been suggested in a study of average Geotail observations presented in Wang et al. ${ }^{10}$ but due to the limited statistics in these TWINS temperatures it is not possible to fully corroborate the Wang et al. results. In the dusk sector, TWINS 1 does show hotter ion temperatures under southward IMF than for northward IMF, as expected, most notably inside of $15 R_{E}$. In the dawn sector, average TWINS 1 ion temperatures and their respective spatial distributions do not show sensitive 
dependence on the orientation of the IMF; this is most likely due to the limited number of instrument sweeps contributing to the temperature maps.

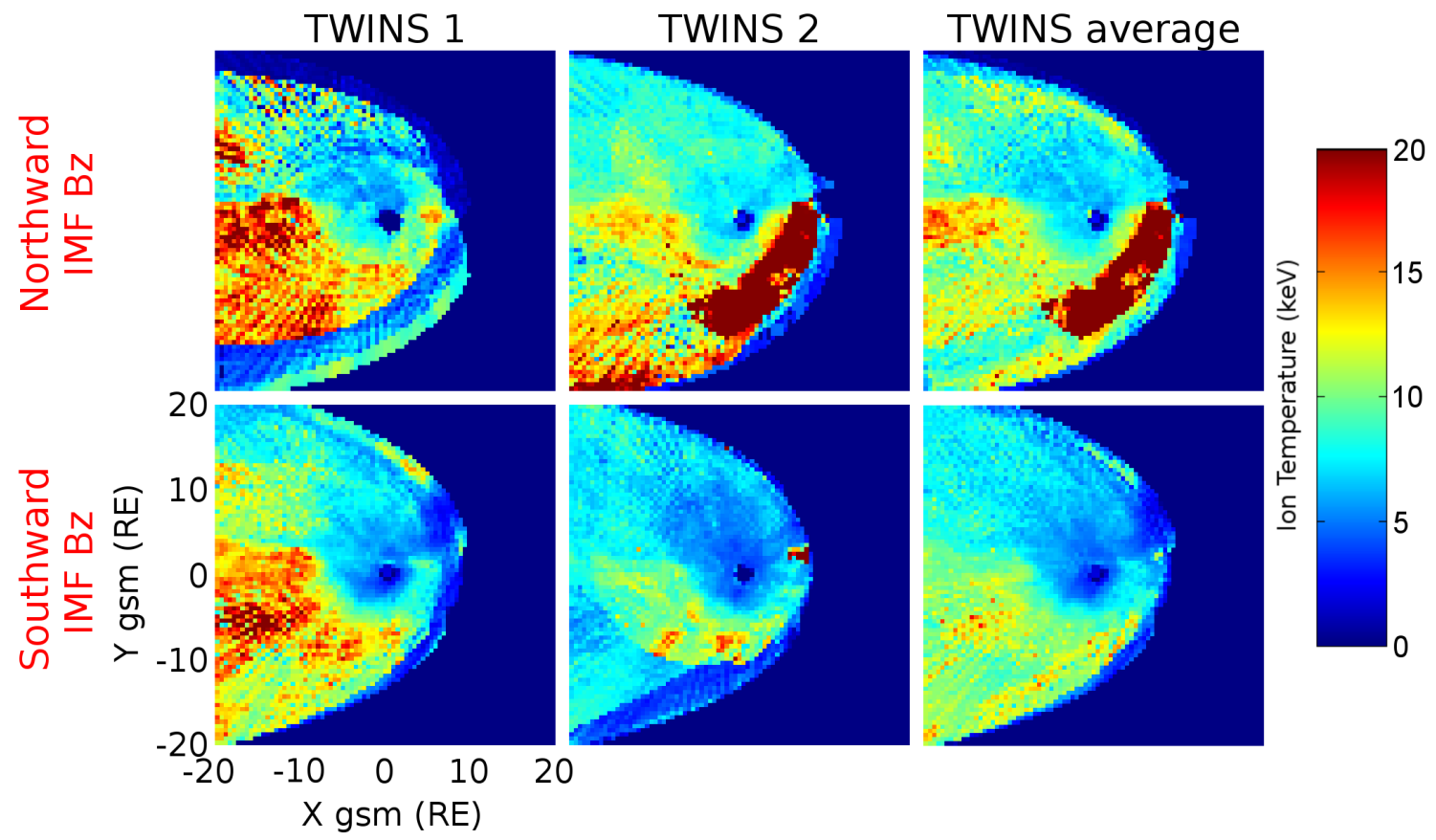

Figure 5.5: Average 2-D ion temperature maps during active periods (Dst $<-30 \mathrm{nT}$ ) for northward $B_{z}$ (left) and southward $B_{z}$ (right) during periods of high solar wind pressure.

For the parameters presented in Figure 5.5, the TWINS 2 temperature maps do not show an obvious head mismatch. During northward IMF however, the TWINS 2 measurements produce artificially high temperatures in the dawn-noon quadrant, the source of which is not currently clear. This effective contamination could not be caused by bad data within a single 30-40 sweep map, since the entire map is built up over 652 total sweeps; abnormally high temperatures from a single interval would regress to a lower, mean temperature after the averaging process. Therefore, this artificially hot region must have been imaged as such for an extended period of time. On the night side of the dawn-dusk meridian, TWINS 2 produces higher overall temperatures for northward IMF than for southward IMF, although 
the temperature maps show no significant difference inside of $10 R_{E}$ in the midnight-dawn quadrant.

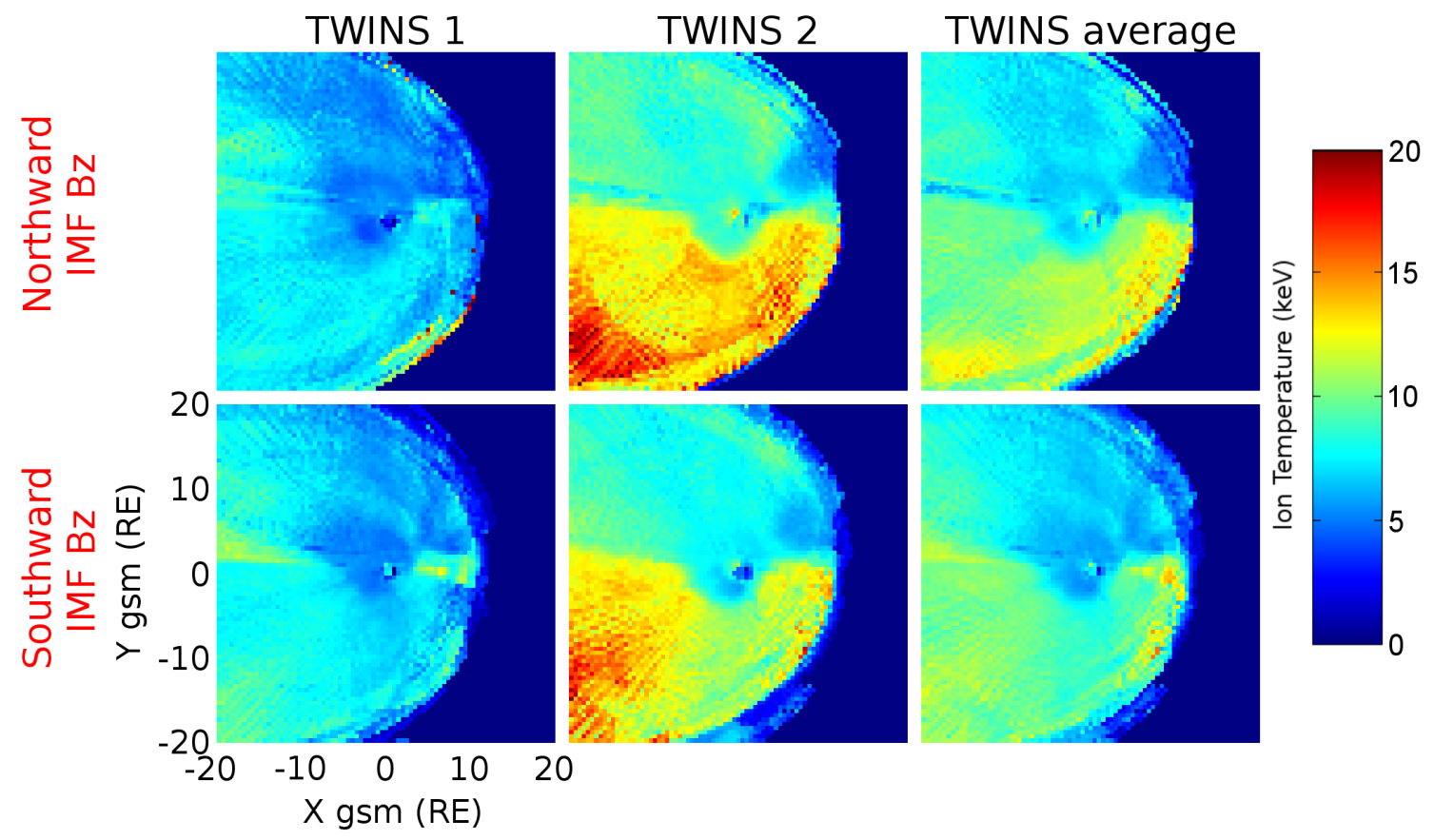

Figure 5.6: Average 2-D ion temperature maps during active periods (Dst $<-30 \mathrm{nT}$ ) for northward $B_{z}$ (left) and southward $B_{z}$ (right) during periods of low solar wind pressure.

In Figure 5.6, the low pressure corollary to the high pressure data in Figure 5.5 is shown, and in the same stylistic format. The temperatures presented in each panel of Figure 5.6 are quite similar to the corresponding panels within Figure 5.4. Refer to the number of instrument actuator sweeps used to calculate each average temperature map, and note that roughly $95 \%$ of the data presented in the panels of Figure 5.4 are taken from intervals with low solar wind pressure. This explains why the two figures are nearly identical. One small difference is that under low pressure only, TWINS 1 measurements indicate a slightly hotter post-midnight distribution between 8-12 $R_{E}$. Temperatures obtained from both TWINS satellites indicate localized heating along the dayside magnetopause under southward IMF 
conditions. These reconnection signatures are found near the magnetopause nose as well as near the pre-noon mean magnetopause location.

Comparing the weighted averages of TWINS 1 and 2 temperature maps for low and high solar wind pressure (Figures 5.6 and 5.5, right panels) it appears that inner magnetospheric ion temperatures exhibit more localized, hot structure during periods of high dynamic pressure. This feature may partially be a result of the disparity between the statistics of the two data sets; the comparatively large low pressure data set serves to average out any anomalous, artificial features that are short-lived in time. Such features can be due, for example, to imperfect fits to the TWINS ENA energy spectra. Also, the time dependence of each satellite's field of view can result in a particular localized region of equatorial pixels being imaged only once or twice before taking the final averages and creating the resulting 2-D ion temperature map. The weighted averages during high pressure also show the dawn-dusk temperature asymmetry orientation as in the low pressure cases imaged by TWINS 1; the weighted averages during intervals of low pressure are not so useful since they factor in data from the severely mismatched TWINS 2 heads. These observed weak asymmetries are mostly in agreement with the Geotail averages presented by Wang et al. 


\section{References}

[1] A. M. Keesee, N. Buzulukova, J. Goldstein, D. J. McComas, E. E. Scime, H. Spence, M. C. Fok, and K. Tallaksen, "Remote observations of ion temperatures in the quiet time magnetosphere," Geophysical Research Letters, vol. 38, no. L03104, pp. 1-5, 2011.

[2] H. Spence and M. Kivelson, "Contributions of the low-latitude boundary layer to the finite width magnetotail convection model," Journal of Geophysical Research, vol. 98, pp. $15487-15496,1993$.

[3] A. M. Zaniewski, X. Sun, A. Gripper, E. E. Scime, J. M. Jahn, and C. J. Pollock, "Evolution of remotely measured inner magnetospheric ion temperatures during a geomagnetic storm," Journal of Geophysical Research, vol. 111, no. A10221, pp. 1-11, 2006.

[4] E. E. Scime and A. M. Keesee, "An analysis of magnetospheric ion temperature as a function of solar wind pressure and north-south magnetic field polarity," in 2013 American Geophysical Union Meeting of the Americas, no. SM43A-05, 2013.

[5] A. M. Keesee, J. G. Elfritz, D. J. McComas, and E. E. Scime, "Superposed epoch analyses of ion temperatures during cme and cir/hss-driven storms," Journal of Atmospheric and Solar-Terrestrial Physics, vol. 115-116, pp. 67-78, 2013.

[6] C. B. Wang, J. K. Chao, and C. H. Lin, "Influence of the solar wind dynamic pressure on the decay and injection of the ring current," Journal of Geophysical Research, vol. 108, no. A9, pp. 1-10, 2003.

[7] Y. Shi, E. Zesta, L. R. Lyons, A. Boudouridis, K. Yumoto, and K. Kitamura, "Effect of 
solar wind pressure enhancements on the storm time ring current asymmetry," Journal of Geophysical Research, vol. 110, no. A10205, p. 1, 2005.

[8] Y. Shi, E. Zesta, L. R. Lyons, A. Boudouridis, K. Yumoto, and K. Kitamura, "Statistical study of effect of solar wind pressure enhancements on the dawn-to-dusk ring current asymmetry," Journal of Geophysical Research, vol. 111, no. A10216, p. 1, 2006.

[9] S. Wing and P. T. Newell, "2d plasma sheet ion density and temperature profiles for northward and southward imf," Geophysical Research Letters, vol. 29, no. 9, p. 1307, 2002.

[10] C. P. Wang, L. R. Lyons, J. M. Weygand, T. Nagai, and R. W. McEntire, "Equatorial distributions of the plasma sheet ions, their electric and magnetic drifts, and magnetic fields under different interplanetary magnetic field bz conditions," Journal of Geophysical Research, vol. 111, no. A04215, 2006. 


\section{Chapter 6}

\section{Conclusions}

The content of this dissertation is fundamentally based on the determination of global magnetospheric ion temperatures from remotely imaged, 2-D energetic neutral atom (ENA) maps by applying the techniques originally discussed by Scime et al. ${ }^{1,2}$ in the context of the IMAGE mission and later by Keesee et al. ${ }^{3}$ using data from the TWINS mission. The ENA maps and derived ion temperature maps discussed herein are provided by the dedicated ENA imagers aboard the two TWINS spacecraft; these instruments offer improved spatial and temporal resolution compared with earlier missions, as well as additional technological improvements. ENA imaging of space plasmas is a relatively young research field, and significant advances have been made over the course of the TWINS mission's lifetime; see Goldstein and McComas. ${ }^{4}$ Because of the resounding and unprecedented successes of the TWINS mission thus far, it is expected that the methods presented in this dissertation will continue to aid space physics researchers in their efforts to untangle complex coupling in the inner magnetosphere. Also, the next generations of space-based ENA imagers will admit even more precision in remote probing of the terrestrial magnetosphere, as well as other planetary magnetospheres and the heliosphere in general. Future instrumental and 
computational advancements in ENA imaging and data processing will continue to provide a wealth of invaluable data that is simply not accessible by means of in situ measurements. A discussion of how the research results presented in this manuscript impact the broader field of inner magnetospheric physics follows, alongside a summary of the results contained herein.

In Chapter 4, results are presented from kinetic, inner magnetospheric simulations where such ion temperature measurements are implemented as outer boundary conditions. This effort constitutes the first time that spatiotemporally resolved ion temperature boundary conditions derived from ENA maps have been supplied to an inner magnetospheric numerical model for the purposes of simulating geomagnetic storms; see Elfritz et al. ${ }^{5}$ for additional details. Thanks to the excellent spatial resolution of the ENA imagers aboard TWINS, users are able to extract well-defined structure within the ion temperature maps. The 2-D temperature maps computed from the corresponding ENA maps are fed into CRCM on timescales typically shorter than 1 hour, and their fundamentally event-specific nature yields modeling opportunities that statistical models based on in situ correlations between solar wind parameters and plasma sheet measurements cannot. One important disadvantage of using empirical plasma sheet models is that in situ observations in the nightside region between geosynchronous orbit and the inner plasma sheet edge are sparse or entirely non-existent. Ion temperature maps derived from TWINS ENA images provide the modeling community with excellent spatial coverage in this critical region, eliminating the need for assumptions and approximations regarding the character of the inner ion plasma sheet. The discussions of where the CRCM boundary is located with respect to this region are found in Sections 4.1.2 and 4.2.2, where it is established that localized processes play an important role in supplying accurate boundary conditions. It is also demonstrated in Chapter 4 that the ion temperature 
boundary conditions have a significant impact on the energy spectra of simulated fluxes and ion moments at geosynchronous orbit and throughout the inner magnetosphere, contrary to findings in earlier works such as Ebihara and Ejiri. ${ }^{6}$ In addition, the data-model comparisons presented for both geomagnetic storms in Chapter 4 indicate that the use of these boundary conditions can better represent transient storm-time features associated with substorm injections, as well as the resulting magnetospheric response to such drivers; refer to Sections 4.1.2 and 4.1.4. In particular, ion temperature moments obtained in situ by THEMIS spacecraft show much closer agreement with simulations which employ the ENA-derived boundary conditions as opposed to simulations driven by the empirical plasma sheet model of Tsyganenko and Mukai. ${ }^{7}$ In Section 4.3, the geosynchronous temperatures determined from the Denton et $a .^{8}{ }^{8}$ superposed epoch analysis are presented in comparison with corresponding temperatures obtained from CRCM simulations in order to elucidate effects associated with the two primary drivers of geomagnetic activity. The late recovery phase heating typical of HSSdriven events is reproduced well by simulations using boundary conditions obtained from TWINS observations. In closing, the ability to incorporate these more realistic ion temperature boundary conditions into inner magnetospheric simulations presents the opportunity to potentially probe ion heating related to substorm activity and other impulsive, transient processes much more effectively. This novel approach to inner magnetospheric modeling will undoubtedly have an impact on future magnetospheric modeling efforts and on the direction of the space weather community as a whole.

Presented in Chapter 5 is the first parametric study correlating average magnetospheric ion temperatures obtained through TWINS ENA images with sets of specific solar wind parameters, IMF parameters, and geomagnetic activity levels near solar maximum. Average temperatures are correlated with various combinations of the Dst index, the solar 
wind dynamic pressure, and the north-south orientation of the IMF $B_{z}$ component. Global magnetospheric ion temperatures positively correlate with decreases in the Dst index, as expected. However, significantly cooler global ion temperatures are observed near the time of minimum Dst. This somewhat counter-intuitive result is explained in proper context in Section 5.2.1. Parametric observations independently and simultaneously binned by solar wind dynamic pressure and IMF $B_{z}$ mostly reproduce the absolute temperature values and storm-time dawn-dusk asymmetries reported by Wang et al. ${ }^{9}$ Wing and Newell, ${ }^{10}$ Shi et al., ${ }^{11,12}$ and Wang et al., ${ }^{13}$ each of which were independently based either on averages of multiple in situ spacecraft measurements in the plasma sheet and inner magnetosphere, ground-based magnetometer measurements mapped to the magnetic equatorial plane, or ionospheric measurements mapped to the magnetic equatorial plane. The results in Chapter 5 suggest that errors associated with the two instrument heads aboard TWINS 2 being mismatched (and TWINS 1, to a lesser degree) may cause non-negligible contamination of ion temperature maps. Since each TWINS satellite orbit is three-axis stabilized, it may be that a single sensor head is always responsible for imaging a single half of the magnetospheric plane; any small deviations in collimator voltages or overall energy sensitivity between the two sensor heads could be the fundamental cause. This may require different background subtraction techniques for each sensor head aboard each TWINS satellite. In future studies it may also be necessary to either manually find and remove contributing temperature maps which display significant head mismatching or to constrain analyses of specific intervals to each head independently, as done in portions of Chapter 5. Overall, the analysis presented in Chapter 5 provides validation for the methods described in Chapter 3 for determining average magnetospheric ion temperatures from global ENA maps, although continued efforts to assess and potentially reduce the statistical errors associated with these remote imaging 
techniques should serve to provide the community with higher levels of confidence regarding the observations. 


\section{References}

[1] E. E. Scime, A. M. Keesee, J. M. Jahn, J. L. Kline, C. J. Pollock, and M. Thomsen, "Remote ion temperature measurements of earth's magnetosphere: Medium energy neutral atom (mena) images," Geophysical Research Letters, vol. 29, no. 10, p. 1438, 2002.

[2] E. E. Scime and A. M. Zaniewski, "Charge exchange imaging of space plasmas," Review of Scientific Instruments, vol. 75, no. 10, pp. 3526-3530, 2004.

[3] A. M. Keesee, N. Buzulukova, J. Goldstein, D. J. McComas, E. E. Scime, H. Spence, M. C. Fok, and K. Tallaksen, "Remote observations of ion temperatures in the quiet time magnetosphere," Geophysical Research Letters, vol. 38, no. L03104, pp. 1-5, 2011.

[4] J. Goldstein and D. J. McComas, "Five years of stereo magnetospheric imaging by twins," Space Science Reviews, vol. 180, pp. 39-70, 2013.

[5] J. G. Elfritz, A. M. Keesee, N. Buzulukova, M. C. Fok, and E. E. Scime, "First results using twins-derived ion temperature boundary conditions in crcm," Journal of Geophysical Research: Space Physics, vol. 119, 2014.

[6] Y. Ebihara and M. Ejiri, "Simulation study on fundamental properties of the stormtime ring current," Journal of Geophysical Research, vol. 105, no. A7, pp. 15843-15859, 2000.

[7] N. A. Tsyganenko and T. Mukai, "Tail plasma sheet models derived from geotail particle data," Journal of Geophysical Research, vol. 108, no. A3, p. 1136, 2003. 
[8] M. H. Denton, J. E. Borovsky, R. M. Skoug, M. F. Thomsen, B. Lavraud, M. G. Henderson, R. L. McPherron, J. C. Zhang, and M. W. Liemohn, "Geomagnetic storms driven by icme- and cir-dominated solar wind," Journal of Geophysical Research, vol. 111, p. A07S07, 2006.

[9] C. P. Wang, L. R. Lyons, J. M. Weygand, T. Nagai, and R. W. McEntire, "Equatorial distributions of the plasma sheet ions, their electric and magnetic drifts, and magnetic fields under different interplanetary magnetic field bz conditions," Journal of Geophysical Research, vol. 111, no. A04215, 2006.

[10] S. Wing and P. T. Newell, "2d plasma sheet ion density and temperature profiles for northward and southward imf," Geophysical Research Letters, vol. 29, no. 9, p. 1307, 2002.

[11] Y. Shi, E. Zesta, L. R. Lyons, A. Boudouridis, K. Yumoto, and K. Kitamura, "Effect of solar wind pressure enhancements on the storm time ring current asymmetry," Journal of Geophysical Research, vol. 110, no. A10205, p. 1, 2005.

[12] Y. Shi, E. Zesta, L. R. Lyons, A. Boudouridis, K. Yumoto, and K. Kitamura, "Statistical study of effect of solar wind pressure enhancements on the dawn-to-dusk ring current asymmetry," Journal of Geophysical Research, vol. 111, no. A10216, p. 1, 2006.

[13] C. B. Wang, J. K. Chao, and C. H. Lin, "Influence of the solar wind dynamic pressure on the decay and injection of the ring current," Journal of Geophysical Research, vol. 108, no. A9, pp. 1-10, 2003. 